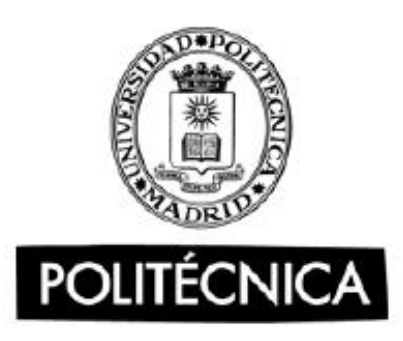

PROGRAMA DE DOCTORADO

DEPARTAMENTO DE ARQUITECTURA, CONSTRUCCIÓN Y SISTEMAS OCEÁNICOS Y NAVALES

ESCUELA TÉCNICA SUPERIOR DE INGENIEROS NAVALES

UNIVERSIDAD POLITÉCNICA DE MADRID

\title{
Modelado y análisis del comportamiento hidro- elástico de rotores para DAECS
}

Autor: D. Alejandro Blanco Medina Ingeniero Naval

Director: D. Amable López Piñeiro

Dr. Ingeniero Naval 
ÍNDICE DE CONTENIDOS

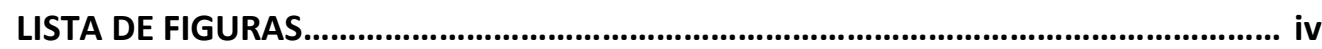

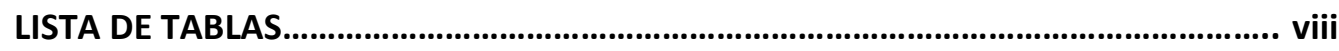

TABLA DE SÍMBOLOS............................................................................................ xii

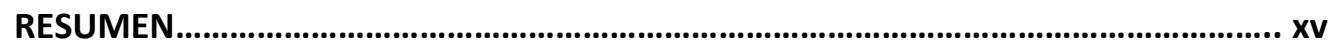

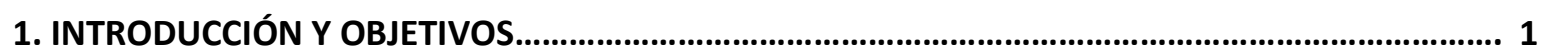

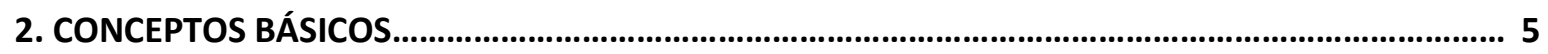

2.1. TEOREMA DE BETZ. (COEFICIENTE DE POTENCIA $\mathrm{c}_{\mathrm{P}}$ ) )................................................ 5

2.2. SISTEMAS Y PARTES PRINCIPALES DE UN GENERADOR DE ELECTRICIDAD DE CORRIENTES CON TURBINA DE EJE HORIZONTAL................................................................... 7

2.3. HERRAMIENTAS UTILIZADAS DE LA MECÁNICA DE FLUIDOS.................................... 10

2.4. PERFILES SUSTENTADORES............................................................................................... 11

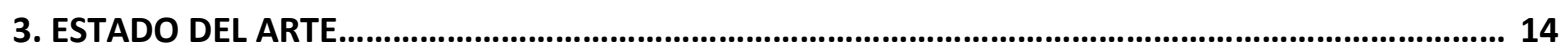

3.1. VENTAJAS E INCONVENIENTES DE LOS PROCEDIMIENTOS DE CÁLCULO

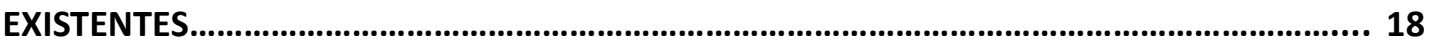

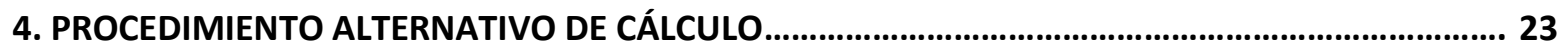

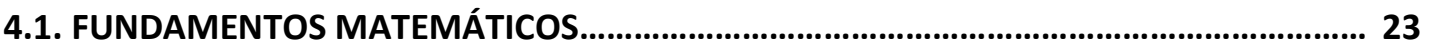

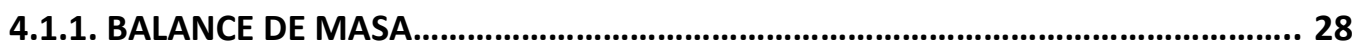

4.1.2. BALANCE DE MOMENTO LINEAL........................................................................... 29

4.1.3. BALANCE DE ENERGÍA ......................................................................................... 32

4.1.4. APLICACIÓN DE LA TEORÍA DE ELEMENTOS DE

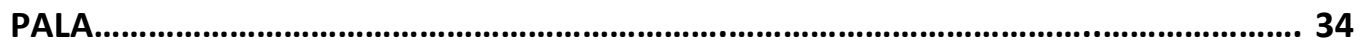

4.1.5. COEFICIENTE DE POTENCIA ........................................................................... 38

4.1.6. RESOLUCIÓN DE LAS ECUACIONES..................................................................... 40

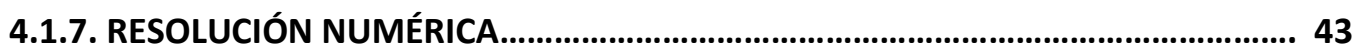

4.1.7.1. ESQUEMA DE PASOS PARA LA RESOLUCIÓN NUMÉRICA..................... 45 


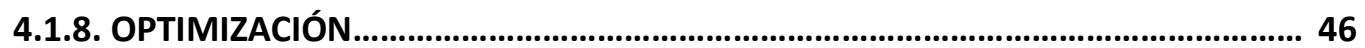

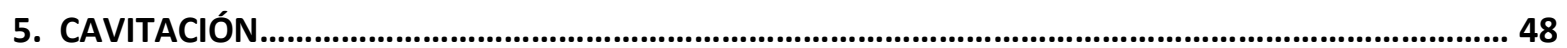

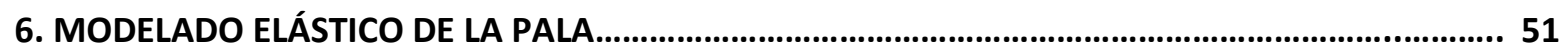

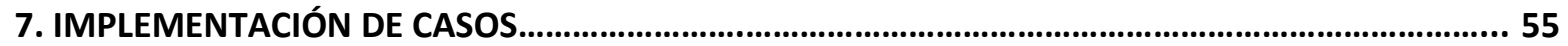

7.1. EJEMPLO DE IMPLEMENTACIÓN PRELIMINAR 1............................................................ 56

7.2. EJEMPLO DE IMPLEMENTACIÓN PRELIMINAR 2......................................................... 59

7.3. TURBINA DE 6,75 METROS DE DIÁMETRO DEL ROTOR.............................................63 63

7.4. TURBINA DE 20 METROS DE DIÁMETRO_................................................................ 72

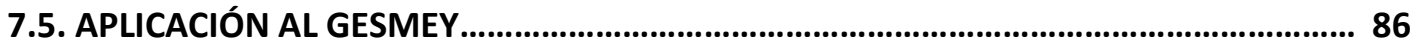

7.5.1 CÁLCULO DE LAS CARGAS INDUCIDAS EN LA PALA PARA EL GESMEY GSYA6.5

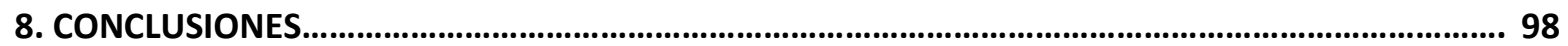

9. BIBLIOGRAFÍA

ANEXO I. CÁLCULO DE LAS FUERZAS DE SUSTENTACIÓN Y ARRASTRE PARA EL CASO DE UN ALA DE DIMENSIÓN FINITA

ANEXO II. FIGURAS DEL APARTADO 7.5.1 PARA EL ESTUDIO DE LA TORSIÓN DE LAS SECCIONES DE PALA 


\section{LISTA DE FIGURAS}

\begin{tabular}{|c|c|c|}
\hline Figura 1 & $\begin{array}{c}\text { Esquema de la relación entre las } \\
\text { fuentes de energía mecánica, } \\
\text { calorífica y electricidad }\end{array}$ & 1 \\
\hline Figura 2 & Esquema para el Teorema de Betz [3] & 5 \\
\hline Figura 3 & Partes del rotor [5] & 8 \\
\hline Figura 4 & $\begin{array}{l}\text { Esquema de las distintas las partes } \\
\text { eléctrica y mecánica de un rotor [5] }\end{array}$ & 9 \\
\hline Figura 5 & $\begin{array}{c}\text { Regulación de pérdida hidrodinámica } \\
\text { activa y pasiva [6] }\end{array}$ & 10 \\
\hline Figura 6 & $\begin{array}{l}\text { Partes geométricas principales de un } \\
\text { perfil sustentador [9] }\end{array}$ & 12 \\
\hline Figura 7 & $\begin{array}{l}\text { Esquema de fuerzas originadas en el } \\
\text { perfil sustentador [9] }\end{array}$ & 12 \\
\hline Figura 8 & $\begin{array}{l}\text { Perfil de presiones y velocidades } \\
\text { axiales en función de la distancia al } \\
\text { disco actuador [13] }\end{array}$ & 15 \\
\hline Figura 9 & $\begin{array}{l}\text { Relación entre velocidad del fluido y } \\
\text { profundidad que puede producir } \\
\text { cavitación [15] }\end{array}$ & 17 \\
\hline Figura 10 & $\begin{array}{c}\text { Representación clásica de un modelo } \\
\text { de disco actuador [24] }\end{array}$ & 19 \\
\hline Figura 11 & $\begin{array}{l}\text { Modelado 3D de un flujo creado por } \\
\text { la técnica de CFD [36] }\end{array}$ & 24 \\
\hline Figura 12 & $\begin{array}{l}\text { Modelado 3D de un flujo creado por } \\
\text { la técnica de CFD [37] }\end{array}$ & 25 \\
\hline Figura 13 & $\begin{array}{l}\text { Modelado 3D de un flujo creado por } \\
\text { la técnica de CFD [35] }\end{array}$ & 25 \\
\hline Figura 14 & $\begin{array}{c}\text { Esquema principal del planteamiento } \\
\text { del problema }\end{array}$ & 26 \\
\hline Figura 15 & $\begin{array}{c}\text { Campo de velocidades originado por } \\
\text { la pala a su paso }\end{array}$ & 27 \\
\hline
\end{tabular}




\begin{tabular}{|c|c|c|}
\hline Figura 16 & $\begin{array}{l}\text { Esquema de las velocidades que } \\
\text { interviene en el problema }\end{array}$ & 28 \\
\hline Figura 17 & $\begin{array}{c}\text { Distribución típica de los coeficientes } \\
\text { de sustentación y arrastre [9] }\end{array}$ & 34 \\
\hline Figura 18 & $\begin{array}{l}\text { Disposición de las fuerzas de } \\
\text { sustentación y de arrastre }\end{array}$ & 35 \\
\hline Figura 19 & $\begin{array}{l}\text { Espiral para la optimización de la } \\
\text { geometría de la pala }\end{array}$ & 46 \\
\hline Figura 20 & $\begin{array}{c}\text { Gráfica tipo de representación del } \\
\text { coeficiente de potencia con respecto } \\
\text { a la TSR [16] }\end{array}$ & 47 \\
\hline Figura 21 & $\begin{array}{l}\text { Gráficas tabuladas de cavitación para } \\
\text { distintos perfiles sustentadores [46] }\end{array}$ & 48 \\
\hline Figura 22 & Distintos tipos de cavitación [21] & 49 \\
\hline Figura 23 & $\begin{array}{l}\text { Disposición esquemática de } \\
\text { refuerzos en la pala [54] }\end{array}$ & 51 \\
\hline Figura 24 & $\begin{array}{l}\text { Punto de aplicación de las cargas en } \\
\text { cada sección }\end{array}$ & 52 \\
\hline Figura 25 & $\begin{array}{c}\text { Representación gráfica de la } \\
\text { distribución de ángulos de ataque } \gamma_{k} \\
\operatorname{rpm}=107, U=7 \mathrm{~m} / \mathrm{s}\end{array}$ & 57 \\
\hline Figura 26 & $\begin{array}{l}\text { Representación gráfica de las } \\
\text { distribuciones radiales de } \\
\text { coeficientes de sustentación y } \\
\text { arrastre } \mathrm{rpm}=107, \mathrm{U}=7 \mathrm{~m} / \mathrm{s}\end{array}$ & 58 \\
\hline Figura 27 & $\begin{array}{l}\text { Representación gráfica de las } \\
\text { distribuciones radiales de cargas } l_{k} y \\
\qquad q_{k} \mathrm{rpm}=107, \mathrm{U}=7 \mathrm{~m} / \mathrm{s}\end{array}$ & 58 \\
\hline Figura 28 & $\begin{array}{l}\text { Representación gráfica de la } \\
\text { distribución de ángulos de ataque } \gamma_{k} \\
\mathrm{rpm}=76, \mathrm{U}=6 \mathrm{~m} / \mathrm{s}\end{array}$ & 60 \\
\hline Figura 29 & $\begin{array}{l}\text { Representación gráfica de las } \\
\text { distribuciones radiales de } \\
\text { coeficientes de sustentación y } \\
\text { arrastre } \mathrm{rpm}=76, \mathrm{U}=2 \mathrm{~m} / \mathrm{s}\end{array}$ & 60 \\
\hline
\end{tabular}




\begin{tabular}{|c|c|c|}
\hline Figura 30 & $\begin{array}{l}\text { Representación gráfica de las } \\
\text { distribuciones radiales de cargas } l_{k} y \\
q_{k} \mathrm{rpm}=76, \mathrm{U}=2 \mathrm{~m} / \mathrm{s}\end{array}$ & 61 \\
\hline Figura 31 & $\begin{array}{l}\text { Representación gráfica de } \varphi_{k}, \lambda\left(r_{k}\right) \\
\text { y } \mu\left(r_{k}\right) \text { con respecto al radio de cada } \\
\text { sección de pala }\end{array}$ & 61 \\
\hline Figura 32 & $\begin{array}{l}\text { Gráfica comparativa de las cargas } \\
\text { estáticas } l_{k} \text { que se producen en la } \\
\text { pala para } \mathrm{rpm}=76, \mathrm{U}=2 \mathrm{~m} / \mathrm{s}\end{array}$ & 62 \\
\hline Figura 33 & $\begin{array}{l}\text { Gráfica comparativa de las cargas } \\
\text { estáticas } q_{k} \text { que se producen en la } \\
\text { pala para } \mathrm{rpm}=76, \mathrm{U}=2 \mathrm{~m} / \mathrm{s}\end{array}$ & 63 \\
\hline Figura 34 & $\begin{array}{c}\text { Representación gráfica de la } \\
\text { distribución radial de coeficientes de } \\
\text { sustentación para } r p m=76, U=2 \\
\mathrm{~m} / \mathrm{s}\end{array}$ & 63 \\
\hline Figura 35 & $\begin{array}{l}\text { Representación gráfica de } \varphi_{k}, \lambda\left(r_{k}\right) \\
\text { y } \mu\left(r_{k}\right) \text { con respecto al radio de cada } \\
\text { sección de pala }\end{array}$ & 66 \\
\hline Figura 36 & $\begin{array}{c}\text { Representación gráfica de la } \\
\text { distribución radial de coeficientes de } \\
\text { sustentación para } \mathrm{rpm}=24, U=1,7 \\
\mathrm{~m} / \mathrm{s}\end{array}$ & 68 \\
\hline Figura 37 & $\begin{array}{l}\text { Gráfica comparativa de las cargas } \\
\text { estáticas } l_{k} \text { que se producen en la } \\
\text { pala para } \mathrm{rpm}=24, \mathrm{U}=1,7 \mathrm{~m} / \mathrm{s}\end{array}$ & 70 \\
\hline Figura 38 & $\begin{array}{l}\text { Gráfica comparativa de las cargas } q_{k} \\
\text { que se producen en la pala para rpm } \\
\qquad=24, U=1,7 \mathrm{~m} / \mathrm{s}\end{array}$ & 70 \\
\hline Figura 39 & $\begin{array}{c}\text { Representación de las distribuciones } \\
\text { de ángulos de ataque geométricos y } \\
\text { cuerdas [28] }\end{array}$ & 73 \\
\hline Figura 40 & $\begin{array}{l}\text { Representación de la distribución de } \\
\text { ángulos de paso geométrico } \beta_{k}\end{array}$ & 74 \\
\hline Figura 41 & $\begin{array}{l}\text { Representación de la distribución de } \\
\text { cuerdas } c_{k}\end{array}$ & 75 \\
\hline Figura 42 & $\begin{array}{l}\text { Representación gráfica de } \varphi_{k}, \lambda\left(r_{k}\right) \\
\text { y } \mu\left(r_{k}\right) \text { con respecto al radio de cada } \\
\text { sección de pala }\end{array}$ & 76 \\
\hline
\end{tabular}




\begin{tabular}{|c|c|c|}
\hline Figura 43 & $\begin{array}{l}\text { Gráfica comparativa de las fuerzas } l_{k} \\
\text { para } \mathrm{rpm}=11,5 \text { y } U=2,4 \mathrm{~m} / \mathrm{s}\end{array}$ & 78 \\
\hline Figura 44 & $\begin{array}{c}\text { Gráfica comparativa de las fuerzas } q_{k} \\
\text { para } \mathrm{rpm}=11,5 \text { y } U=2,4 \mathrm{~m} / \mathrm{s}\end{array}$ & 79 \\
\hline Figura 45 & $\begin{array}{c}\text { Gráfica comparativa de las fuerzas } l_{k} \\
\text { para } \mathrm{rpm}=9 \mathrm{y} U=2,4 \mathrm{~m} / \mathrm{s}\end{array}$ & 81 \\
\hline Figura 46 & Aspecto de la pala en estudio & 84 \\
\hline Figura 47 & $\begin{array}{l}\text { Estructura interior de la pala } \\
\text { compuesta por un refuerzo principal } \\
\text { circular y extremo de pala macizo }\end{array}$ & 85 \\
\hline Figura 48 & $\begin{array}{c}\text { Estructura interior de la pala } \\
\text { compuesta por un refuerzo de } \\
\text { sección cuadrada y extremo de pala } \\
\text { macizo }\end{array}$ & 85 \\
\hline Figura 49 & $\begin{array}{l}\text { Gráfica comparativa de las } \\
\text { distribuciones de coeficientes de } \\
\text { sustentación a lo largo de la pala } \\
\text { obtenidas con las distintas } \\
\text { metodologías implementadas para } \\
\text { rpm }=12, U=2 \mathrm{~m} / \mathrm{s}\end{array}$ & 91 \\
\hline Figura 50 & $\begin{array}{l}\text { Gráfica comparativa de las } \\
\text { distribuciones de cargas } l_{k} \text { a lo largo } \\
\text { de la pala obtenidas con las distintas } \\
\text { metodologías implementadas para } \\
\qquad \mathrm{rpm}=12, \mathrm{U}=2 \mathrm{~m} / \mathrm{s}\end{array}$ & 92 \\
\hline Figura 51 & $\begin{array}{l}\text { Gráfica comparativa de las } \\
\text { distribuciones de cargas } q_{k} \text { a lo largo } \\
\text { de la pala obtenidas con las distintas } \\
\text { metodologías implementadas para } \\
\qquad \mathrm{rpm}=12, \mathrm{U}=2 \mathrm{~m} / \mathrm{s}\end{array}$ & 92 \\
\hline
\end{tabular}




\section{LISTA DE TABLAS}

\begin{tabular}{|c|c|c|}
\hline Tabla 1 & $\begin{array}{c}\text { Tabla comparativa de diferentes } \\
\text { características de las principales } \\
\text { fuentes de energía }\end{array}$ & 2 \\
\hline Tabla 2 & $\begin{array}{l}\text { Tabla comparativa de los métodos de } \\
\text { cálculo utilizados en el ámbito de las } \\
\text { turbinas de corrientes marinas }\end{array}$ & 22 \\
\hline Tabla 3 & $\begin{array}{c}\text { Coeficiente de proporcionalidad K } \\
\text { para distintas geometrías de sección } \\
\text { de viga [52] }\end{array}$ & 53 \\
\hline Tabla 4 & $\begin{array}{l}\text { Número de palas recomendable en } \\
\text { función de la velocidad TSR de diseño } \\
\text { [14] }\end{array}$ & 56 \\
\hline Tabla 5 & $\begin{array}{c}\text { Geometría de pala de la turbina en } \\
\text { estudio }\end{array}$ & 57 \\
\hline Tabla 6 & $\begin{array}{l}\text { Distribuciones de ángulos de ataque } \\
\qquad \gamma_{k} \mathrm{rpm}=107, \mathrm{U}=7 \mathrm{~m} / \mathrm{s}\end{array}$ & 57 \\
\hline Tabla 7 & $\begin{array}{l}\text { Parámetros de geometría de pala } \\
\text { implementados }\end{array}$ & 59 \\
\hline Tabla 8 & $\begin{array}{l}\text { Distribuciones de ángulos de ataque, } \\
\text { coeficientes de sustentación y } \\
\text { arrastre, } r p m=76, U=2 \mathrm{~m} / \mathrm{s}\end{array}$ & 59 \\
\hline Tabla 9 & $\begin{array}{c}\text { Geometría de pala implementada y } \\
\text { variables auxiliares }\end{array}$ & 61 \\
\hline Tabla 10 & $\begin{array}{c}\text { Distribuciones de } \gamma_{\mathrm{k}} \text { para } \mathrm{rpm}=76, \mathrm{U} \\
=2 \mathrm{~m} / \mathrm{s}\end{array}$ & 62 \\
\hline Tabla 11 & Parámetros principales del rotor [51] & 64 \\
\hline Tabla 12 & $\begin{array}{l}\text { Características geométricas de la pala } \\
\text { [51] }\end{array}$ & 64 \\
\hline Tabla 13 & $\begin{array}{l}\text { Parámetros principales de } \\
\text { funcionamiento del rotor } \\
\text { implementados }\end{array}$ & 65 \\
\hline Tabla 14 & $\begin{array}{l}\text { Datos implementados de las } \\
\text { características geométricas de la pala }\end{array}$ & 65 \\
\hline
\end{tabular}




\begin{tabular}{|c|c|c|}
\hline Tabla 15 & $\begin{array}{l}\text { Valores de las variables auxiliares } \varphi_{\mathrm{k}}, \\
\qquad \lambda\left(\mathbf{r}_{\mathrm{k}}\right) \text { y } \mu\left(\mathbf{r}_{\mathrm{k}}\right)\end{array}$ & 66 \\
\hline Tabla 16 & $\begin{array}{c}\text { Distribuciones de } \gamma_{\mathrm{k}} \text { para } \mathrm{rpm}=24, \mathrm{U} \\
=1,7 \mathrm{~m} / \mathrm{s}\end{array}$ & 67 \\
\hline Tabla 17 & $\begin{array}{l}\text { Distribuciones de } \gamma_{k} \text { rpm = } 24 \text { con el } \\
\text { método de elementos de pala [20] }\end{array}$ & 68 \\
\hline Tabla 18 & $\begin{array}{c}\text { Nueva geometría de pala } \\
\text { implementada y ángulos de ataque } \\
\gamma_{k} \text { obtenidos con el procedimiento } \\
\text { alternativo }\end{array}$ & 69 \\
\hline Tabla 19 & $\begin{array}{c}\text { Nueva geometría de pala } \\
\text { implementada y ángulos de ataque } \\
\gamma_{k} \text { obtenidos con el método de } \\
\text { elementos de pala rpm }=24, \mathrm{U}=\mathbf{1 , 7} \\
\mathrm{m} / \mathrm{s} .\end{array}$ & 69 \\
\hline Tabla 20 & $\begin{array}{c}\text { Nueva geometría de pala } \\
\text { implementada y ángulos de ataque } \\
\gamma_{k} \text { obtenidos con el procedimiento } \\
\text { pala } \mathrm{rpm}=20, \mathrm{U}=1,7 \mathrm{~m} / \mathrm{s} .\end{array}$ & 71 \\
\hline Tabla 21 & $\begin{array}{c}\text { Nueva geometría de pala } \\
\text { implementada y ángulos de ataque } \\
\gamma_{k} \text { obtenidos con el método de } \\
\text { elementos de pala para rpm }=20, \mathrm{U}= \\
1,7 \mathrm{~m} / \mathrm{s} \text {. }\end{array}$ & 71 \\
\hline Tabla 22 & $\begin{array}{c}\text { Parámetros principales de } \\
\text { funcionamiento del rotor [28] }\end{array}$ & 72 \\
\hline Tabla 23 & $\begin{array}{l}\text { Parámetros de funcionamiento del } \\
\text { rotor en el procedimiento de cálculo }\end{array}$ & 72 \\
\hline Tabla 24 & Definición de la geometría de pala & 74 \\
\hline Tabla 25 & $\begin{array}{l}\text { Valores de las variables auxiliares } \varphi_{\mathrm{k}}, \\
\qquad \lambda\left(\mathbf{r}_{\mathrm{k}}\right) \text { y } \mu\left(\mathrm{r}_{\mathrm{k}}\right)\end{array}$ & 76 \\
\hline Tabla 26 & $\begin{array}{l}\text { Distribución de ángulos de ataque } \gamma_{\mathrm{k}} \\
\text { y coeficientes de sustentación y } \\
\text { arrastre obtenidas con la } \\
\text { implementación del método de } \\
\text { elementos de pala para } \mathrm{rpm}=\mathbf{1 1 , 5}\end{array}$ & 77 \\
\hline Tabla 27 & $\begin{array}{l}\text { Nueva geometría de pala modificada } \\
\text { por rotación } 16^{\circ} \text { de los ángulos de } \\
\text { paso geométricos y la distribución de } \\
\text { ángulos de ataque } \gamma_{\mathrm{k}} \text { para rpm=11,5 }\end{array}$ & 78 \\
\hline
\end{tabular}




\begin{tabular}{|c|c|c|}
\hline Tabla 28 & $\begin{array}{l}\text { Nueva distribución de los ángulos de } \\
\text { ataque } \gamma_{k} \text { para rpm }=9 \text { y } U=2,4 \mathrm{~m} / \mathrm{s}\end{array}$ & 80 \\
\hline Tabla 29 & $\begin{array}{l}\text { Nueva distribución de los ángulos de } \\
\text { ataque } \gamma_{\mathrm{k}} \text { para } \mathrm{rpm}=9 \text { y } \mathrm{U}=2,4 \mathrm{~m} / \mathrm{s} \\
\text { (BET) }\end{array}$ & 81 \\
\hline Tabla 30 & $\begin{array}{l}\text { Nueva distribución de los ángulos de } \\
\text { ataque teniendo en cuenta el efecto } \\
\text { de la torsión en las secciones de pala } \\
\text { para rpm = } 11,5 \text { y considerando un } \\
\text { refuerzo principal de sección circular }\end{array}$ & 82 \\
\hline Tabla 31 & $\begin{array}{c}\text { Nueva distribución de los ángulos de } \\
\text { ataque teniendo en cuenta el efecto } \\
\text { de la torsión en las secciones de pala } \\
\text { para rpm = } 11,5 \text { y considerando un } \\
\text { refuerzo principal de sección } \\
\text { cuadrada }\end{array}$ & 83 \\
\hline Tabla 32 & $\begin{array}{c}\text { Parámetros principales de } \\
\text { funcionamiento de los diseños GSY- } \\
\text { A6.5 y GSY U1M [61] }\end{array}$ & 86 \\
\hline Tabla 33 & $\begin{array}{c}\text { Parámetros principales de } \\
\text { funcionamiento del GSY-A6.5 } \\
\text { implementados para el } \\
\text { procedimiento de cálculo }\end{array}$ & 87 \\
\hline Tabla 34 & $\begin{array}{l}\text { Geometría de pala definida para el } \\
\text { GSY-A6.5 en } 20 \text { secciones }\end{array}$ & 88 \\
\hline Tabla 35 & $\begin{array}{l}\text { Variables auxiliares } \lambda \text { y } \mu \text {, y la función } \\
\varphi \text { para cada una de las secciones }\end{array}$ & 89 \\
\hline Tabla 36 & $\begin{array}{l}\text { Distribución de ángulos de ataque, } \\
\text { coeficientes de sustentación y } \\
\text { arrastre para la condición nominal de } \\
\text { funcionamiento del GSY-A6.5, rpm = } \\
\qquad 12 \text { y U }=2.0 \mathrm{~m} / \mathrm{s}\end{array}$ & 90 \\
\hline Tabla 37 & $\begin{array}{l}\text { Distribución de ángulos de ataque, } \\
\text { coeficientes de sustentación y } \\
\text { arrastre para la condición nominal de } \\
\text { funcionamiento del GSY-A6.5, rpm = } \\
\qquad 12 \text { y U = 2. } 0 \mathrm{~m} / \mathrm{s} \text { (BET) }\end{array}$ & 91 \\
\hline Tabla 38 & $\begin{array}{l}\text { Módulo de Young y Poisson para } \\
\text { distintos materiales [54] }\end{array}$ & 95 \\
\hline Tabla 39 & $\begin{array}{l}\text { Distribución de los momentos de } \\
\text { torsión y ángulos de torsión en la pala }\end{array}$ & 96 \\
\hline
\end{tabular}



aluminio 


\section{TABLA DE SÍMBOLOS}

\begin{tabular}{|c|c|}
\hline$P$ & Potencia extraída por el rotor \\
\hline$\rho$ & Densidad del fluido \\
\hline$v$ & Velocidad media del fluido que atraviesa el rotor \\
\hline$S$ & Sección transversal de la turbina \\
\hline$v_{1}$ & Velocidad del fluido a la entrada del rotor \\
\hline$v_{2}$ & Velocidad del fluido a la salida del rotor \\
\hline$f$ & Relación de velocidades, $\frac{\mathrm{v}_{2}}{\mathrm{v}_{1}}$ \\
\hline$t$ & Variable tiempo en la ecuación. \\
\hline$l$ & Variable longitud en la ecuación \\
\hline$E_{c}$ & Energía cinética \\
\hline$U$ & Velocidad de la corriente \\
\hline$c_{p}$ & Coeficiente de potencia, $\mathrm{C}_{\mathrm{P}}=\frac{\mathrm{P}}{\frac{1}{2} \rho \mathrm{SU}^{3}}$ \\
\hline$S$ & $\begin{array}{l}\text { Letra designada para definir la frontera que delimita una determinada } \\
\text { región espacial en el seno de un fluido }\end{array}$ \\
\hline$V$ & Letra que representa el volumen de fluido delimitado por la frontera S \\
\hline $\bar{v}$ & Vector velocidad del fluido en un determinado punto del espacio \\
\hline$\overline{d s}$ & Vector elemento diferencial de superficie \\
\hline$c$ & Cuerda de un perfil sustentador \\
\hline$c_{L}$ & Coeficiente de sustentación \\
\hline$c_{D}$ & Coeficiente de arrastre \\
\hline$L$ & Fuerza de sustentación en perfil bidimensional, $\mathrm{L}=\frac{1}{2} \rho \mathrm{c}_{\mathrm{L}} \mathrm{cU}^{2}$ \\
\hline$D$ & Fuerza de arrastre en perfil bidimensional, $\mathrm{D}=\frac{1}{2} \rho \mathrm{c}_{\mathrm{D}} \mathrm{CU}^{2}$ \\
\hline$\omega$ & Velocidad angular de giro del rotor \\
\hline$a$ & Radio del núcleo del rotor \\
\hline$b$ & Radio del rotor \\
\hline TSR & $\begin{array}{l}\text { Relación entre la velocidad de la pala del rotor en su extremo y la } \\
\text { velocidad de la corriente, } \frac{\omega b}{U}\end{array}$ \\
\hline$\sigma$ & Coeficiente o número de cavitación \\
\hline
\end{tabular}




\begin{tabular}{|c|c|}
\hline$P_{0}$ & Presión atmosférica \\
\hline$P_{v}[T]$ & Presión de vapor a la temperatura T \\
\hline$h$ & Profundidad \\
\hline$\Gamma$ & Intensidad de circulación \\
\hline$r$ & Variable radial \\
\hline$z$ & Variable axial \\
\hline$\theta$ & Variable angular \\
\hline $\bar{u}_{r}$ & Vector unitario en la dirección $r$ \\
\hline $\bar{u}_{z}$ & Vector unitario en la dirección $z$ \\
\hline $\bar{u}_{\theta}$ & Vector unitario en la dirección $\theta$ \\
\hline$v_{r}$ & $\begin{array}{l}\text { Componente radial de la velocidad en la estela de pala, } \\
\qquad \mathrm{v}_{\mathrm{r}}=\frac{\Gamma}{2 \pi}\left[\frac{\mathrm{z}}{(\mathrm{r}-\mathrm{a})^{2}+\mathrm{z}^{2}}-\frac{\mathrm{z}}{(\mathrm{r}-\mathrm{b})^{2}+\mathrm{z}^{2}}\right]\end{array}$ \\
\hline$v_{z}$ & $\begin{array}{l}\text { Componente en la dirección } \mathrm{z} \text { en la estela de pala, } \\
\qquad \mathrm{v}_{\mathrm{z}}=\frac{\Gamma}{2 \pi}\left[-\frac{\mathrm{r}-\mathrm{a}}{(\mathrm{r}-\mathrm{a})^{2}+\mathrm{z}^{2}}+\frac{\mathrm{r}-\mathrm{b}}{(\mathrm{r}-\mathrm{b})^{2}+\mathrm{z}^{2}}\right]\end{array}$ \\
\hline$v_{\omega r}^{*}$ & Velocidad rotacional de salida del fluido en la estela de pala \\
\hline$U^{*}$ & Velocidad axial de salida del fluido en la estela de pala \\
\hline$Z$ & Número de palas del rotor \\
\hline$\varphi(r)$ & $\begin{array}{l}\text { Variable auxiliar, } \\
\qquad \varphi(r)=\arctan \left[\frac{\pi \mathrm{a}}{\mathrm{Z}(\mathrm{b}-\mathrm{r})}\right]+\arctan \left[\frac{\pi \mathrm{a}}{\mathrm{Z}(\mathrm{r}-\mathrm{a})}\right]\end{array}$ \\
\hline$F_{r}$ & Componente de la fuerza en la dirección radial \\
\hline$F_{z}$ & Componente de la fuerza en la dirección axial \\
\hline$F_{\theta}$ & Componente de la fuerza en la dirección angular \\
\hline$M$ & $\begin{array}{l}\text { Momento resultante de las fuerzas que actúan en la pala en el eje del } \\
\text { rotor }\end{array}$ \\
\hline$\mu(r)$ & $\begin{array}{l}\text { Variable auxiliar, } \\
\qquad \mu(\mathrm{r})=\int_{-\frac{\pi \mathrm{a}}{\mathrm{Z}}}^{\frac{\pi \mathrm{a}}{\mathrm{Z}}}\left(\frac{\mathrm{z}}{(\mathrm{r}-\mathrm{a})^{2}+\mathrm{z}^{2}}-\frac{\mathrm{z}}{(\mathrm{r}-\mathrm{b})^{2}+\mathrm{z}^{2}}\right)^{2} \mathrm{dz}\end{array}$ \\
\hline$\lambda(r)$ & $\begin{array}{l}\text { Variable auxiliar, } \\
\qquad \lambda(\mathrm{r})=\int_{-\frac{\pi \mathrm{a}}{\mathrm{Z}}}^{\frac{\pi \mathrm{a}}{\mathrm{z}}}\left(-\frac{\mathrm{r}-\mathrm{a}}{(\mathrm{r}-\mathrm{a})^{2}+\mathrm{z}^{2}}+\frac{\mathrm{r}-\mathrm{b}}{(\mathrm{r}-\mathrm{b})^{2}+\mathrm{z}^{2}}\right)^{2} \mathrm{dz}\end{array}$ \\
\hline
\end{tabular}




\begin{tabular}{|c|c|}
\hline$V$ & $\begin{array}{l}\text { Velocidad del fluido en las cercanías de cada sección de pala, } \\
\qquad V \cong \sqrt{U^{2}+\omega^{2} r^{2}}\end{array}$ \\
\hline$q$ & $\begin{array}{l}\text { Componente angular de la fuerza en cada sección de pala, } \\
\qquad \mathrm{q}=\frac{1}{2} \rho \mathrm{cV}^{2}\left(\mathrm{c}_{\mathrm{L}} \cos \alpha-\mathrm{c}_{\mathrm{D}} \operatorname{sen} \alpha\right)\end{array}$ \\
\hline$l$ & $\begin{array}{l}\text { Componente de la fuerza axial en cada sección de pala, } \\
\qquad l=\frac{1}{2} \rho \operatorname{cV}^{2}\left(c_{\mathrm{L}} \operatorname{sen} \alpha+\mathrm{c}_{\mathrm{D}} \cos \alpha\right)\end{array}$ \\
\hline$\beta$ & Ángulo que forma cada sección de pala con la horizontal \\
\hline$\alpha$ & Ángulo que forma la velocidad Vcon la horizontal \\
\hline$\gamma$ & Ángulo de ataque de la sección, $\gamma=\beta-\alpha$ \\
\hline$\gamma_{s}$ & Angulo de pérdida \\
\hline$\delta r$ & Longitud radial de sección de pala \\
\hline$\psi(r)$ & $\begin{array}{l}\text { Variable auxiliar, } \\
\qquad \psi(\mathrm{r})=\frac{1}{2} \mathrm{cV}^{2}\left[\mathrm{c}_{\mathrm{L}} \operatorname{sen}(\beta-\gamma)+\mathrm{c}_{\mathrm{D}} \cos (\beta-\gamma)\right]-\frac{\Gamma}{\pi} \mathrm{v}_{\omega \mathrm{r}}^{*} \varphi(\mathrm{r})-\frac{2 \pi \mathrm{a}}{\mathrm{Z}}\left(\mathrm{U}^{* 2}-\mathrm{U}^{2}\right)\end{array}$ \\
\hline$c_{P}{ }^{*}$ & $\begin{array}{l}\text { Coeficiente de potencia máximo teórico, } \\
\qquad \mathrm{c}_{\mathrm{P}}{ }^{*}=\frac{\mathrm{Z} \omega \int_{\mathrm{a}}^{\mathrm{b}} \mathrm{rcV}^{2}\left(\mathrm{c}_{\mathrm{L}} \cos \alpha\right) \mathrm{dr}}{\pi \mathrm{b}^{2} \mathrm{U}^{3}}\end{array}$ \\
\hline$G$ & $\begin{array}{l}\text { Módulo de rigidez del material, depende del Módulo de Young E y el } \\
\text { Coeficiente de Poisson } v, G=\frac{E}{2(1+v)}\end{array}$ \\
\hline Q & Momento de torsión en la sección de pala \\
\hline$\beta^{\prime}$ & Ángulo de paso geométrico de pala sometida a torsión \\
\hline$\theta$ & Ángulo resultante de la deformación por torsión \\
\hline$\varepsilon_{1}$ & Coeficiente de pérdidas por punta de pala \\
\hline$\varepsilon_{2}$ & Coeficiente de pérdidas por extremo de núcleo \\
\hline$\varepsilon$ & Coeficientes global de pérdidas, $\varepsilon=\varepsilon_{1} \varepsilon_{2}$ \\
\hline$s_{1}$ & Factor de inducción axial \\
\hline$s_{2}$ & Factor de inducción angular \\
\hline
\end{tabular}




\title{
Resumen
}

La transformación de la energía cinética del mar en una fuente renovable de energía eléctrica podría ser un hecho relevante desde el punto de visto tecnológico en un futuro cercano. En el campo de las energías renovables marinas, las turbinas de corrientes de eje horizontal son posiblemente el dispositivo más adecuado para la obtención de electricidad. Las técnicas de cálculo existentes en la actualidad para obtener las distribuciones de cargas a las que está sometida una pala de una turbina de corrientes son las siguientes: Modelos de disco actuador, Método de líneas sustentadoras y método de elementos finitos. Estas técnicas presentan ciertas dificultades para su implementación y obtención de resultados preliminares, ya que entrañan gran complejidad conceptual y su entendimiento no resulta sencillo ni tampoco elaborar procedimientos de cálculo que se puedan utilizar durante la fase de diseño conceptual o básico de una turbina. En este trabajo, se analizarán los conceptos físicos y matemáticos que intervienen en el funcionamiento de una turbina de corrientes, para confeccionar un método o procedimiento de cálculo que sea relativamente intuitivo y sencillo de manejar. Esta herramienta de cálculo permitirá obtener resultados para el diseño básico, y también puede ser un punto de partida para manejar otras herramientas de cálculo más avanzadas ya que los principios fundamentales son los mismos. En la parte final del trabajo se aplicarán las ecuaciones obtenidas a varios casos reales de turbinas de corrientes.

\begin{abstract}
Transforming the kinetic energy of the sea into renewable useful source electricity could be a relevant technologic development in the near future. In this field, marine horizontal axis turbines (HAT) are possibly, the most important devices. The current calculation techniques used to obtain the acting forces or loads in the marine current horizontal axis rotor blades: Actuator disc models, lifting lines and finite element method, have certain disadvantages which lead difficulties to obtain relatively quick results, in addition, these methods are not easy to understand and mastering, and furthermore, complex computing tools are required to obtain results. In the present work, the common physical and mathematic fundamentals will be studied and analyzed with the aim to create a new easier managing calculating tool. The proposed procedure of this document can be considered as a help towards to calculate preliminary solutions easier, and also a starting point to manage physical ideas which could be used with other calculation tools. And finally, the mathematic fundamentals will be explained and the implementation of the equations in a real case. Nevertheless, due to the real complexity of the problem, the assumed simplifications of this model must be taken into account, in order to know the possible calculating limitations of this procedure and consequently use it correctly.
\end{abstract}




\section{INTRODUCCIÓN Y OBJETIVOS}

Desde hace más de un siglo, la electricidad consumida en casas, oficinas e industria se ha producido en plantas de generación térmicas, en centrales nucleares o bien en centrales hidroeléctricas. Con una fuerte dependencia de los combustibles fósiles, en cualquiera de sus formas (carbón, petróleo y derivados, gas, etc.) que componen cerca del $74 \%$ de la energía consumida total [1].

Con la entrada en el siglo XXI, debido a la mayor concienciación existente acerca de la conservación de la naturaleza y el cambio climático, se está produciendo un replanteamiento de las fuentes de energía eléctrica utilizadas convencionalmente. Haciendo que, en los últimos años, se haya puesto el foco de atención en las energías renovables y dentro de éstas, empieza a tener gran interés por sus ventajas, el aprovechamiento de la energía que mueve los mares y océanos, con corrientes y mareas.

Desde un punto de vista termodinámico, los océanos se comportan como un proceso termomecánico que convierte la energía irradiada por el sol y la derivada debido a interacciones gravitatorias en energía mecánica en forma de movimiento de masas de agua. Siendo la densidad energética de la mareas es del orden de 4 veces superior densidad energética de la energía eólica y 30 veces a la solar [1].

Un observador extraterrestre, que haya estado atento el devenir tecnológico de la humanidad, puede llegar a no entender como el ser humano ha sido capaz de dominar procesos tan complicados como los que ocurren en una máquina de combustión interna o un reactor nuclear $y$, sin embargo, o no ha sido de su interés o no ha sabido qué hacer con las fuentes de energía mecánica o de trabajo existentes (viento, olas, corrientes, mareas, etc.) , aun siendo, algo mucho más eficiente, directo y lógico, para la obtención de algo útil y fundamental para el ser humano contemporáneo como es la electricidad. En la Figura 1 se refleja esquemáticamente esta idea.

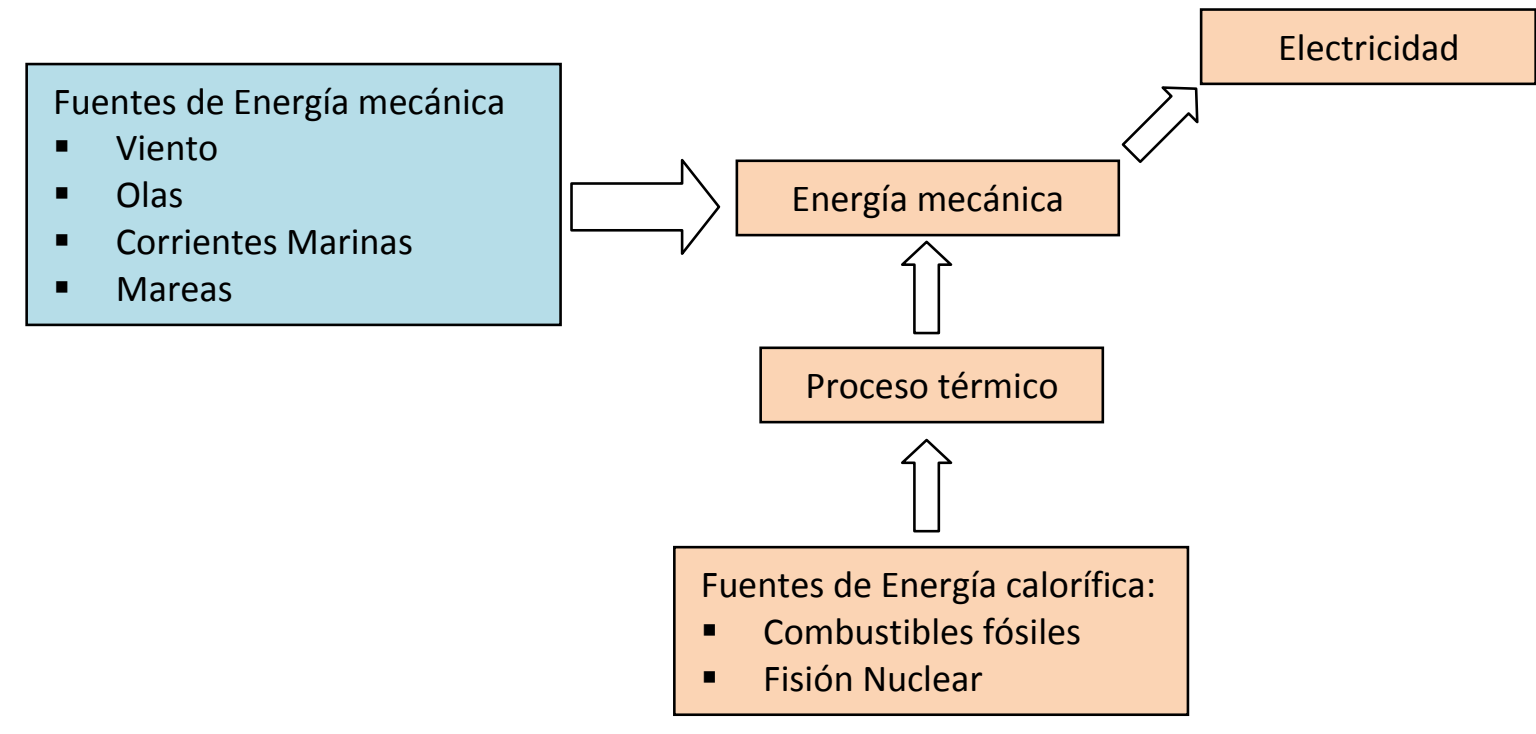

Figura 1. Esquema de la relación entre las fuentes de energía mecánica, calorífica y electricidad 
Asimismo, ante la inminente crisis de los combustibles fósiles con sus efectos contaminantes y los desastres medioambientales que ha protagonizado la energía nuclear en los últimos años, el aprovechamiento de fuentes de energía inagotables como las que ofrecen los océanos va cobrando mayor importancia para la sociedad. Muchos países, en estos momentos, empiezan a contemplar las energías renovables marinas como un elemento estratégico en sus planes de futuro.

Por lo que, todas las barreras científico-tecnológicas que existían, se están empezando a sortear, hasta este momento, en el que el desarrollo de las tecnologías de conversión de estas energías en electricidad ha alcanzado un punto en el que parece cada vez más consistente la idea de que se produzca, en los próximos años, el aporte de energía eléctrica procedente de dispositivos de aprovechamiento de la energía de corrientes y mareas a nivel comercial.

Si se realiza una tabla comparativa en la que aparezcan las principales fuentes de energía, con algunas características como pueden ser la densidad de energía, las emisiones, impacto visual, etc. Se puede comprobar que la energía basada en el mar es la que mejores resultados ofrece (Tabla 1).

\begin{tabular}{|c|c|c|c|c|c|}
\hline $\begin{array}{c}\text { Fuente de } \\
\text { energía }\end{array}$ & $\begin{array}{c}\text { Corrientes } \\
\text { Marinas/Olas }\end{array}$ & $\begin{array}{c}\text { Combustibles } \\
\text { fósiles }\end{array}$ & $\begin{array}{c}\text { É́lica on- } \\
\text { shore }\end{array}$ & $\begin{array}{c}\text { Eólica off- } \\
\text { shore }\end{array}$ & Solar \\
\hline $\begin{array}{c}\text { Densidad de } \\
\text { energía }\end{array}$ & Alta & Muy alta & Baja & Media-baja & Baja \\
\hline Variabilidad & Baja & Ninguna & Alta & Media & Media \\
\hline Emisiones & Ninguna & $\begin{array}{c}\text { Carbon, NOx, } \\
\text { SO2. Otros. }\end{array}$ & Ninguna & Ninguna & Ninguna \\
\hline Impacto visual & Mínimo & Alto & Medio & Medio-bajo & Medio-alto \\
\hline
\end{tabular}

Tabla 1. Tabla comparativa de diferentes características de las principales fuentes de energía

El aprovechamiento de las energías basadas en el movimiento de masas de agua en el mar se puede realizar de varias formas [2]: extracción de energía de las olas, de las mareas, de las corrientes o de las diferencias de salinidad que puede existir en determinadas zonas.

Y concretamente, la tecnología basada en la extracción de energía a partir de las corrientes marinas ha ido ganando importancia en el sector marítimo. Ya que, las corrientes marinas en determinadas zonas estratégicas son muy predecibles y esto facilita enormemente, desde el punto de vista técnico, la producción de energía eléctrica a gran escala.

La mejora de la capacidad de las computadoras actuales, así como el mayor conocimiento de los fenómenos que ocurren en sistemas fluidos, han permitido en los últimos años la elaboración de modelos matemáticos muy precisos que permiten conocer con gran detalle el funcionamiento de una turbina de corrientes mucho antes de que se construya.

Sin embargo, las técnicas de cálculo clásicas que se han implementado en materia de turbinas de eje horizontal, siguen siendo muy utilizadas, ya que su consumo de recursos es mínimo y son de gran importancia en la fase de diseño básico o preliminar.

El estudio hidrodinámico, en la fase de diseño básico o preliminar, del rotor de una turbina es, como se ha dicho, esencial en el proceso de diseño. Puesto que, de este modo se pueden descartar geometrías de rotor, para las que se haya determinado que no tienen las características de 
funcionamiento que se esperaba, pero con la ventaja de no haber invertido gran cantidad de tiempo y recursos para este primer análisis cualitativo

Y es esta tesitura en la que se desarrolla esta tesis, en vista de las expectativas técnicas y comerciales de esta fuente de energía, en la que se buscará aportar una metodología de cálculos preliminar o bien mejorar las técnicas existentes este ámbito, que permita optimizar el comportamiento hidrodinámico y elástico del rotor de una turbina, de corrientes marinas de eje horizontal (En inglés recibe el acrónimo "HATT","Horizontal Axis Tidal Turbine").

De aquí sobreviene el título: "Modelado y análisis del comportamiento Hidro-elástico de rotores para DAECS", donde el acrónimo DAECS corresponde a: Dispositivos de Aprovechamiento de Energía de Corrientes Submarinas.

El planteamiento general es el siguiente: Se trata de conocer cuál será el comportamiento de una turbina de corrientes marinas, para una determinada velocidad de la corriente de mar, tanto desde el punto de vista energético, como estructural.

Para abordar este problema, por tanto, el primer paso consiste en recopilar información acerca de: Modelos hidrodinámicos, cavitación, resistencia y fatiga, etc. que se puedan aplicar a este tipo de generador de corrientes y con arreglo a ellos establecer una metodología que permita realizar un análisis cualitativo de las características que pueden mejorar o empeorar su rendimiento o el tiempo de vida.

Una vez se haya recopilado y asimilado toda la información, se hará un análisis tratando de determinar cuáles son las ventajas e inconvenientes de las técnicas o modelos de cálculo utilizadas y en consecuencia, tratar de aportar mejoras en los puntos que se pueda.

En materia de energías renovables marinas, las publicaciones o documentos, así como de otros dispositivos de aprovechamiento de la energía del mar, no aparecen hasta finales de la década de 1990 y hasta la actualidad.

Este hecho, se debe en gran medida a que durante la mayor parte del siglo pasado, el desarrollo tecnológico y social se ha basado principalmente en el consumo de combustibles fósiles, y es en el último tercio del siglo cuando se empieza a producir cierta conciencia de que estos combustibles tienen efectos negativos graves sobre el medio ambiente además de ser una fuente de energía que se agotará en un determinado momento.

La mejora de las tecnologías relacionadas con la electrónica y la informática ha hecho posible que se resuelvan problemas endémicos de la generación de electricidad mediante dispositivos que aprovechan energías renovables, ya que se han dotado a éstos, de sistemas que permiten controlar parámetros de funcionamiento como puede ser la velocidad de giro de una turbina, así se facilitaría el acople eléctrico a una red de distribución, también pueden proteger la unidad al activarse ciertos sistemas en caso de que se produzcan condiciones meteorológicas adversas que pongan en riesgo la integridad del dispositivo. 
Siendo estos aspectos, solamente ejemplos de los innumerables problemas técnicos que la electrónica y la informática han ayudado a solucionar en la última década, y se espera que cada vez la capacidad de las aplicaciones sea cada vez mayor en los próximos años.

Uno de los problemas más importantes que se han encontrado a la hora de confeccionar este trabajo ha sido la enorme dispersión en cuanto a nomenclatura, conceptos utilizados, etc. de los artículos y otros documentos relacionados con esta materia.

Por ello, el trabajo principal ha sido el de estudiar los fundamentos teóricos en profundidad para poder discernir qué material puede ser de utilidad para cumplir con los objetivos planteados inicialmente para esta tesis, para poder encontrar puntos en los que se pueda aportar una mejora. 


\section{CONCEPTOS BÁSICOS}

En este apartado, se van a tratar una serie de conceptos básicos, con el objeto de facilitar la comprensión de los desarrollos que se utilizarán a lo largo de la tesis. En primer lugar, se recoge la demostración del Teorema de Betz, que es el concepto matemático más importante en el funcionamiento de los dispositivos de extracción de energía mecánica.

Seguidamente, se describen los sistemas necesarios de una turbina para la generación de electricidad así como los conceptos necesarios en materia de hidrodinámica y teoría de perfiles sustentadores.

La demostración del Teorema de Betz se basa en el desarrollo teórico de las turbinas eólicas, pero que, puesto que se trata de un planteamiento matemático, no existe ninguna diferencia a la hora de plantear esta demostración para el caso de una turbina de corrientes marinas [3]. También muchos aspectos tecnológicos que se utilizan en las turbinas eólicas se pueden aplicar a las turbinas marinas [4].

Es importante conocer también, los sistemas mecánicos, eléctricos, fenómenos físicos y conceptos termodinámicos que intervienen en el funcionamiento de una turbina de corrientes de eje horizontal. De esta forma, se podrá tener un concepto global de este dispositivo y por tanto, se facilitará la lectura de los apartados sucesivos.

\subsection{TEOREMA DE BETZ. RENDIMIENTO (COEFICIENTE DE POTENCIA $\mathbf{c}_{\mathbf{P}}$ ) [3]}

El Teorema de Betz fue publicado en 1919 por el físico alemán Albert Betz y establece un límite termodinámico máximo a la energía que se puede extraer de un determinado fluido en movimiento, es decir, la relación entre la energía extraída y la del fluido en cualquier dispositivo que aproveche este tipo de energía no podrá superar en ningún caso este límite.

Para calcular esta cota superior se partirá del siguiente esquema, representado en la Figura 2, que puede ser identificado con una turbina de eje horizontal o bien con cualquier otro dispositivo que genere energía a partir de una corriente de un fluido.

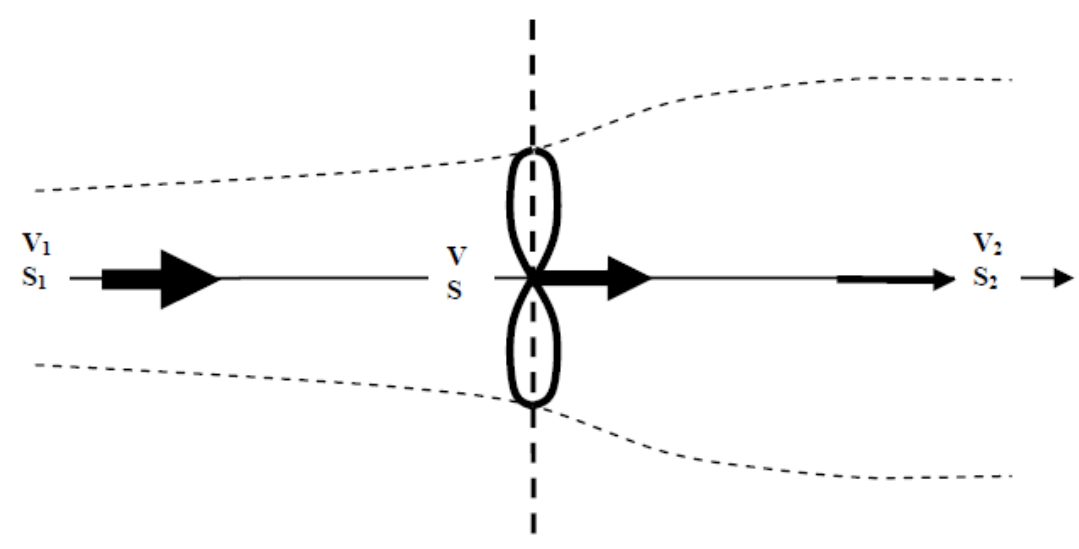

Figura 2. Esquema para el Teorema de Betz [3] 
En base a este esquema se puede establecer el siguiente balance de energía,

$$
P=\frac{\rho v S}{2}\left(v_{1}^{2}-v_{2}^{2}\right)
$$

Donde $P$, es la potencia que se aprovecha en el rotor de la turbina y $\rho v S$, es el flujo de masa por unidad de tiempo que atraviesa la superficie de rotor $\mathrm{S}$.

Teniendo en cuenta ahora que:

$v_{2}<v_{1} ; v \cong \frac{v_{1}+v_{2}}{2}$, ya que la corriente perderá velocidad una vez que atraviese la superficie del rotor, y haciendo:

$f=\frac{v_{2}}{v_{1}} \rightarrow v_{2}=f v_{1} \rightarrow 0<f<1$

Sustituyendo en la ecuación Ec. 1, ésta queda de la siguiente forma:

$$
P=\frac{\rho\left(v_{1}+v_{2}\right) S}{4}\left(v_{1}^{2}-v_{2}^{2}\right)=\frac{1}{4} \rho S v_{1}^{3}(1+f)\left(1-f^{2}\right)
$$

La potencia que transporta la corriente, se calcula con el incremento de energía cinética que atraviesa una determinada sección del flujo, durante $\Delta t$.

En un instante $t=0$, la energía cinética que atraviesa la sección es cero, sin embargo, en un instante $t=\delta t$, la corriente habrá avanzado una longitud $\delta l$, y esta energía cinética es:

$$
\Delta E_{c}=\frac{1}{2} \rho S v_{1}^{2} \delta l
$$

Ahora ya se puede calcular la potencia que transporta la corriente, mediante la siguiente expresión:

$$
\frac{\Delta E_{c}}{\Delta t}=\frac{\frac{1}{2} \rho S v_{1}^{2} \delta l}{\delta t}=\frac{1}{2} \rho S v_{1}^{3}
$$

Y de acuerdo a todo lo anterior, el rendimiento o coeficiente de potencia $c_{p}$, del rotor queda determinado por el cociente:

$$
c_{p}=\frac{\frac{1}{4} \rho S v_{1}^{3}(1+f)\left(1-f^{2}\right)}{\frac{1}{2} \rho S v_{1}^{3}}=\frac{1}{2}(1+f)\left(1-f^{2}\right)
$$

Calculando la derivada de $c_{P}$ con respecto a $f$, queda:

$$
\frac{d c_{p}}{d b}=\frac{1}{2}\left(1-f^{2}\right)+\frac{1}{2}(1+f)(-2 f)
$$

Igualando esta derivada a cero: 


$$
\frac{1}{2}\left(1-f^{2}\right)+\frac{1}{2}(1+f)(-2 f)=0
$$

Las soluciones de esta ecuación se obtiene para:

$$
f=-1 ; f=\frac{1}{3}
$$

Como,

Calculando $c_{P}$ :

$$
0<f<1 \rightarrow f=\frac{1}{3}
$$

$$
c_{p_{\left(f=\frac{1}{3}\right)}}=\frac{1}{2}\left(1+\frac{1}{3}\right)\left(1-\frac{1}{3^{2}}\right)=\frac{16}{27}=0,592
$$

Siendo este valor el máximo alcanzable por el rendimiento de una turbina que genera energía a partir de una corriente de un fluido. Este valor se conoce como límite de Betz.

\subsection{SISTEMAS Y PARTES PRINCIPALES DE UN GENERADOR DE ELECTRICIDAD DE CORRIENTES CON TURBINA DE EJE HORIZONTAL}

Las turbinas de corrientes están formadas por varios sistemas y subsistemas [5], compuestos a su vez, por elementos mecánicos, estructurales, eléctricos y electrónicos mediante los cuales, se consigue trasformar la energía cinética de la corriente, en electricidad.

Pueden existir variaciones según sea el diseño de cada fabricante de turbinas, sin embargo, estas son las principales partes esquemáticas, comunes a casi todos ellos.

\section{- Sistema Mecánico}

El sistema mecánico, engloba todos aquellos elementos del generador que no tienen que ver con la generación de electricidad o con el control del mismo, dentro de este sistema existen otros subsistemas:

\section{Subsistema Hidrodinámico (rotor)}

En los generadores de corrientes, la energía se absorbe a través de fuerzas de sustentación hidrodinámicas que se generan al circular la corriente de agua a través de perfiles hidrodinámicos que a su vez conforman palas que se integran en un rotor. El rotor, asimismo está compuesto de eje, núcleo y palas. 


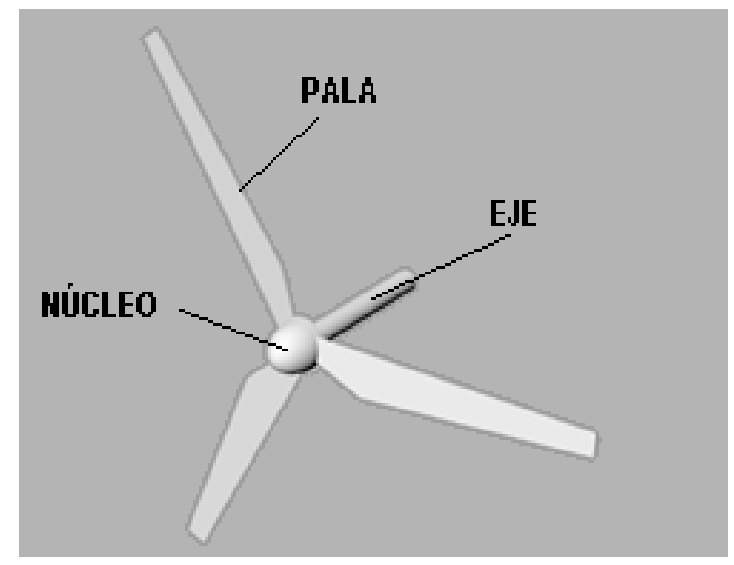

Figura 3. Partes del rotor [5]

\section{- Reductora o multiplicadora}

El movimiento del rotor arrastra un eje, este eje está acoplado a una reductora o multiplicadora (según sea si reduce o aumenta las revoluciones) que permite obtener velocidades de rotación adecuadas para el acople a los sistemas de generación eléctrica.

En la actualidad, ya existen algunos generadores en los que el acoplamiento es directo, es decir, no se instala la reductora.

\section{- Sistema Eléctrico}

En el sistema eléctrico se incluyen todos los elementos relacionados con la generación de electricidad, en él se pueden distinguir los siguientes subsistemas:

\section{- Subsistema de generación eléctrica (Power Take Off "P.T.O.")}

Es aquí donde de se genera la electricidad en forma de señal trifásica que se aportará a la red. Todos los sistemas y subsistemas del generador actúan para que la potencia que entre y salga del alternador esté lo más controlada posible.

Los elementos necesarios para obtener la potencia de forma controlada, es decir, turbina, eje, frenos, reductora o multiplicadora, sistema de control, etc. Reciben la denominación anglosajona: (Power Take Off “P.T.O.”).

\section{- Subsistema de regulación}

Se compone de elementos electrónica de potencia, su función es absorber las irregularidades en la señal eléctrica del generador, para que la ésta tenga la amplitud y frecuencia adecuadas.

La señal que sale directamente de los generadores acoplados al eje del generador, suele ser en ocasiones con forma de diente de sierra, en este caso la misión del sistema de la regulación será transformar esta señal a una sinusoidal, para poder transmitirla a la red. 


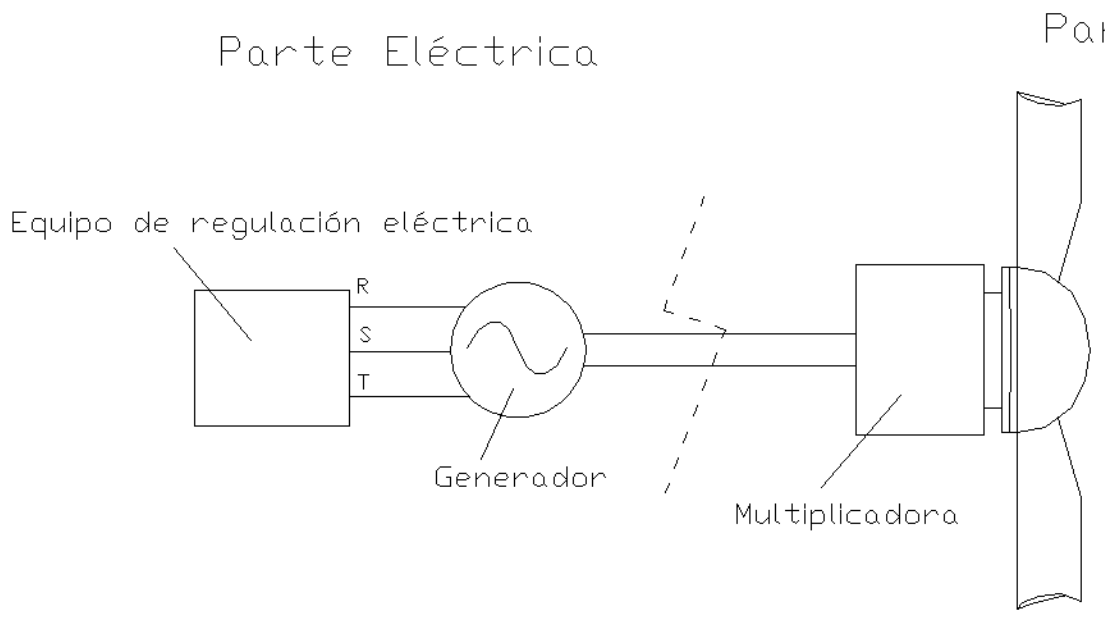

Figura 4. Esquema de las distintas las partes eléctrica y mecánica de un rotor [5]

\section{- Sistema de Control}

El sistema de control trabaja a entre el sistema mecánico y el sistema eléctrico y está formado por un sistema de sensores y actuadores, su función principal es tratar que la potencia que se absorbe en el sistema mecánico sea la adecuada para que se genere una señal eléctrica lo más parecida a la que existe en la red.

Por ejemplo, si el generador lleva un sistema de palas controlables, el sistema de control puede actuar sobre el ángulo de ataque de las palas en aquellos generadores de corrientes que tengan esta posibilidad, con esto se puede conseguir que la variación de revoluciones debida a las fluctuaciones de la corriente sea mínima. Esto se traduce finalmente en que la frecuencia de la señal eléctrica sea lo más constante posible.

El sistema de control, también actuará como protector del generador, deteniendo el funcionamiento del generador si eso fuese necesario, debido a condiciones climatológicas adversas. Conviene reseñar en este punto que en la actualidad existen dos posibilidades de proteger la unidad de generador.

- Subsistema de pasivo de pérdida hidrodinámica. En este caso no existe la posibilidad de variar el paso de las palas. El principio de funcionamiento se debe a la geometría de cada pala, que a determinadas velocidades no produce sustentación alguna (el rotor entra en pérdida hidrodinámica) provocando su detención.

- Subsistema activo de pérdida hidrodinámica. Con este sistema, sí se puede controlar mecánicamente el paso de las palas del rotor, con ello se puede decidir cuándo es conveniente que el rotor se detenga, para evitar posibles averías o incluso la destrucción de unidad. 


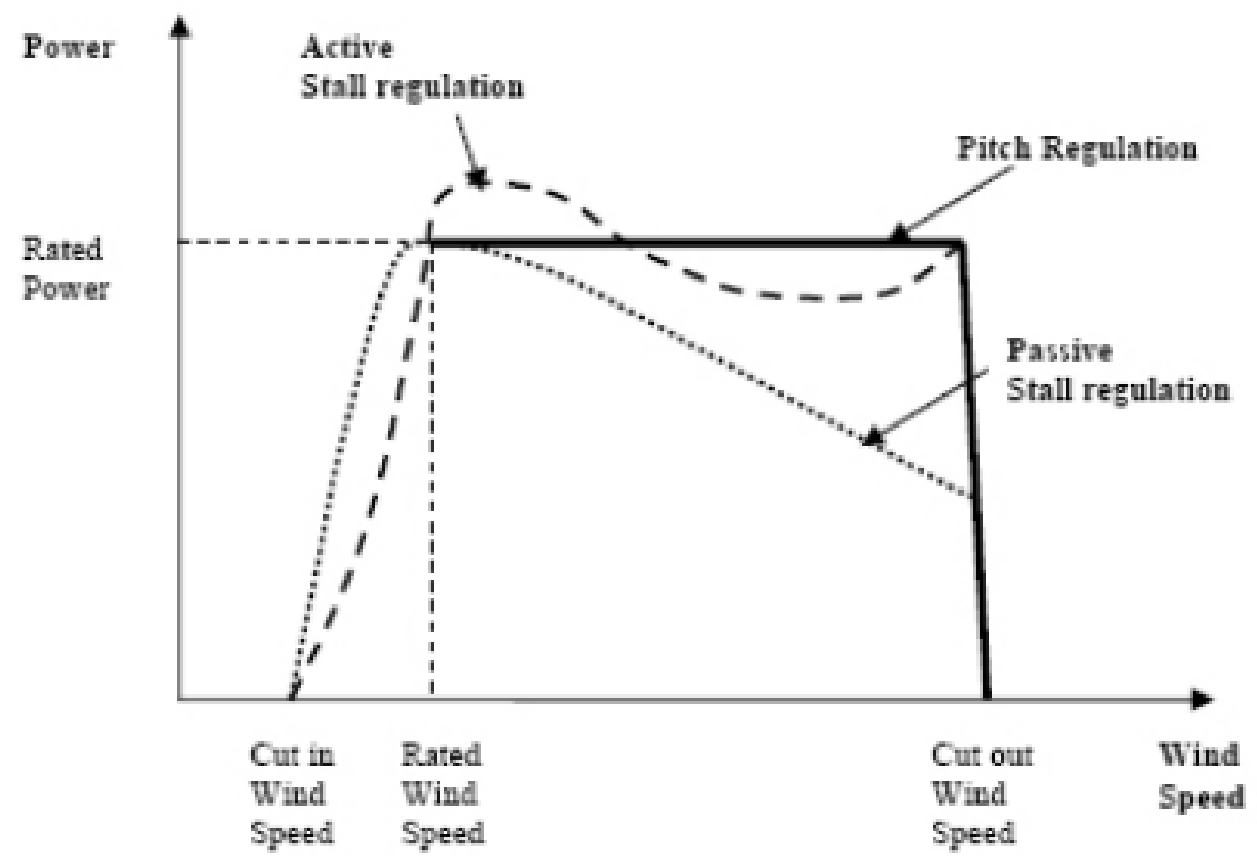

Figura 5. Regulación de pérdida hidrodinámica activa y pasiva [6]

\section{- Elementos de sujeción}

La misión de los elementos de sujeción es mantener unido el generador a un punto fijo que normalmente suele ser un anclaje en el fondo marino. Los elementos de sujeción suelen ser cables de alta resistencia y elementos de unión, diseñados para soportar las cargas estáticas y dinámicas. Junto a ellos va fijado el cable por el que se transmite la electricidad hacia los puntos de conexión a la red, normalmente situados en el fondo marino.

\subsection{HERRAMIENTAS UTILIZADAS DE LA MECÁNICA DE FLUIDOS [7]}

En el desarrollo de esta tesis se utilizarán algunos conceptos matemáticos básicos relacionados con la Mecánica de Fluidos, como son la conservación de la masa, la ecuación del momento lineal y la energía, aplicadas a un determinado volumen $V$ de un fluido en movimiento, de densidad constante $\rho$, delimitado por su superficie exterior $S$ rígida, aunque completamente permeable. Y también con la consideración de que el movimiento del fluido se encuentra en régimen estacionario.

Con este sistema definido, la masa de fluido que contiene el volumen $V$ es constante y por lo tanto el balance másico global a través de la superficie $S$, debe ser nulo, esto se expresa con la ecuación siguiente,

$$
\int_{S} \rho \bar{v} \overline{d s}=0
$$

Donde, $\bar{v}$ es el vector velocidad del fluido en los puntos de $S$, y $\overline{d s}$ es el vector diferencial de superficie. 
En el caso del momento lineal, hay que tener en cuenta, que de acuerdo a las leyes fundamentales de la dinámica, la diferencia de esta magnitud a través de $S$, está producido por fuerzas exteriores que interactúan con el sistema. Esto permite plantear la ecuación del siguiente modo,

$$
\int_{S} \rho \bar{v}(\bar{v} \overline{d s})=\sum F_{\text {ext }}
$$

Esta ecuación es de enorme importancia en el estudio de problemas relacionados con fluidos en movimiento, de aquí se puede deducir Teoremas como el de Bernoulli, y también obtener las fuerzas que intervienen en un determinado problema. Aunque se pueden manejar otras expresiones que representan la misma ecuación, en esta tesis se va a utilizar esta forma para trabajar.

Por último, queda establecer la expresión que rige el balance de energía en el sistema elegido. La deducción, es un poco más compleja que las anteriores y la idea que hay que manejar es la del principio del conservación de la energía.

En el sistema fluido en estudio, la energía entra en forma de energía cinética con las partículas fluidas y sale también de esta forma. La diferencia entre la energía que entra y sale será igual a la energía que se aporta o se extrae del sistema.

Para aportar o extraer energía solamente se consideran, en esta tesis, energías mecánicas y no se tienen en cuenta energías caloríficas. La expresión correspondiente al flujo de energía cinética a través de $S$ es la siguiente,

$$
\int_{S} \rho \frac{1}{2} v^{2}(\bar{v} \overline{d s})
$$

En muchos casos, este flujo de energía cinética es igual al trabajo por unidad de tiempo que realizan las fuerzas exteriores al sistema.

El manejo de estas expresiones requiere sumo cuidado cuando se aplican a casos concretos, ya que normalmente conllevan una gran carga de operaciones y un error en ellas puede impedir la obtención de resultados válidos.

Más adelante se partirá de estas expresiones y se realizará un desarrollo completo aplicado a una pala de un rotor de turbina de corrientes de eje horizontal, donde se podrá observar la complejidad de los cálculos y operaciones a realizar.

\subsection{PERFILES SUSTENTADORES $[8,9,10]$}

Si se tiene en cuenta que la geometría de las palas de turbinas de corrientes, eólicas y otros dispositivos está basada en perfiles sustentadores bidimensionales, cuyas características físicas están estudiadas con gran minuciosidad en documentos especializados, se ve que esta técnica es de gran importancia para cualquier estudio de materias como la que se trata en esta tesis. 
Un perfil sustentador es, desde el punto de vista geométrico, una curva cerrada con forma alargada en una dirección principal, que cuando éste se mueve en el seno de un fluido se originan unas fuerzas que dependen del tamaño del perfil y de la naturaleza del contorno del perfil. La geometría del perfil se puede caracterizar con algunas partes descriptivas típicas que se recogen a continuación:

- Borde de ataque: Es la parte que está enfrentada al flujo del fluido.

- Borde de salida: Es la parte por la que fluido abandona el perfil.

- Cuerda: Se denota con la letra $c$, y es la distancia máxima que existe en puntos de la curva geométrica del perfil.

- Ángulo de ataque: Es el ángulo que forma el vector velocidad del fluido con la línea recta definida por la cuerda $c$.

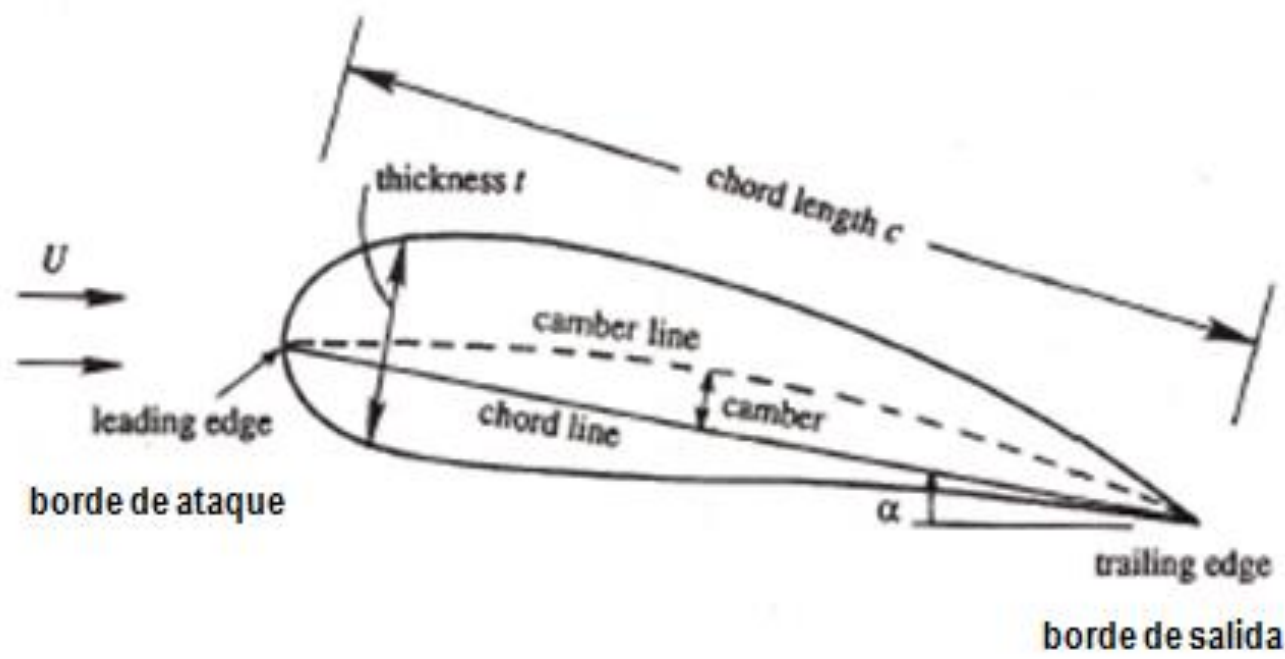

Figura 6. Partes geométricas principales de un perfil sustentador [9]

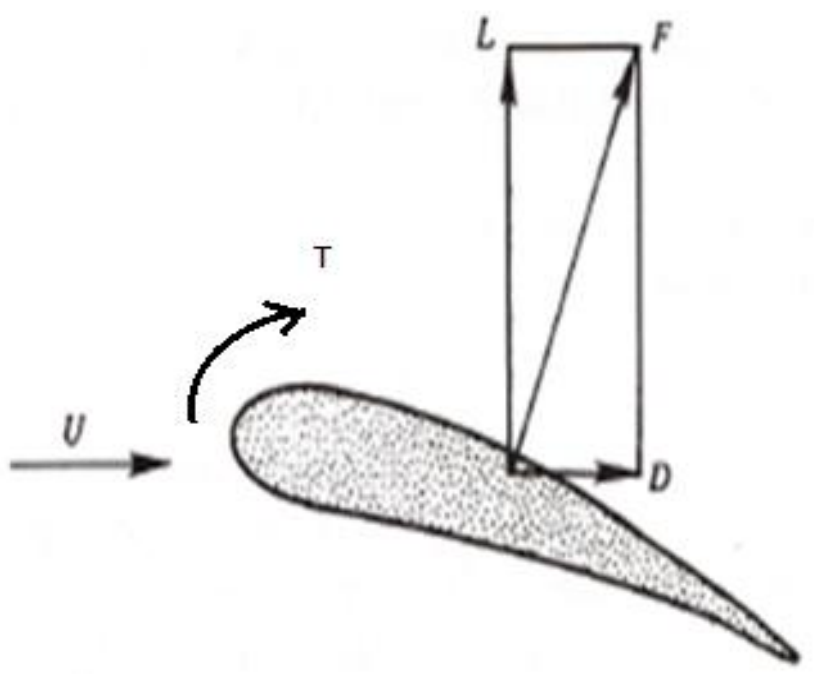

Figura 7. Esquema de fuerzas originadas en el perfil sustentador [9] 
Desde el punto de vista de las fuerzas que se originan en el perfil, al moverse en el seno de un fluido, éstas se descomponen en dos fuerzas principales, una perpendicular a la dirección de movimiento, con sentido hacia la parte superior del perfil, esta fuerza se denomina fuerza de sustentación y se denota por la letra $L$. La otra fuerza que se origina es opuesta a la velocidad del fluido, se denomina fuerza de arrastre y se denota por la letra $D$.

Estas fuerzas se pueden calcular mediante las expresiones,

$$
\begin{aligned}
L & =\frac{1}{2} \rho c_{L} c U^{2} \\
D & =\frac{1}{2} \rho c_{D} c U^{2}
\end{aligned}
$$

Adicionalmente a estas fuerzas, cuando el fluido atraviesa en perfil sustentador, produce un momento o par sobre éste, que se corresponde con la expresión, que tiende a aumentar el ángulo de ataque de la sección,

$$
Q=\frac{1}{2} \rho c_{m} c^{2} U^{2}
$$

En las expresiones anteriores, $c_{L}$ y $c_{D}$ son los coeficientes de sustentación y arrastre respectivamente, y $c_{m}$ el coeficiente de momento o par que se produce en el perfil sustentador, todos estos coeficientes son adimensionales que dependen de la geometría del perfil, y su valor varía entre 0 y 1,2 para el caso del coeficiente de sustentación y entre 0 y 0,05 para el de arrastre.

El valor de los coeficientes $c_{L}, c_{D}$ y $c_{\mathrm{m}}$ se puede encontrar tabulado en varias publicaciones especializadas y dependen de [11]:

- El ángulo de ataque que forma el perfil con respecto a la velocidad del fluido.

- De la geometría del perfil, como se ha dicho antes.

- El número de Reynolds del fluido en movimiento.

Por lo que para obtenerlos, sencillamente se acude a una de estas publicaciones se identifica el perfil concreto sobre el que se esté trabajando y los parámetros adecuados al problema en estudio.

Por otro lado, si se observan las ecuaciones Ec. 14 y Ec. 15 de $L$ y $D$ se puede comprobar que las unidades resultantes son de fuerza por unidad de longitud, es decir, mediante estas expresiones solamente se pueden obtener las fuerzas que se producen sobre un perfil de longitud infinita o un perfil bidimensional. 


\section{ESTADO DEL ARTE}

Si hubiese que recopilar históricamente los documentos especializados en materia de aprovechamiento de energías renovables, quizá el primer documento o publicación a reseñar corresponde al científico alemán Albert Betz, que en 1919 enunciase su famoso Teorema.

A partir de este momento, ya hay que esperar unos años, hasta el último tercio del siglo XX, puesto que en este período se produjeron grandes avances en materia de energías renovables, y sobre todo en el ámbito de las turbinas eólicas.

En las últimas dos décadas, es el momento en el que se empiezan a publicar los primeros documentos relacionados con la investigación de las turbinas de corrientes marinas, llegando hasta la actualidad, momento en que esta materia origina gran de contenidos y de temáticas de investigación.

Muchas de estas publicaciones recientes están dedicadas a las técnicas de cálculo utilizadas en turbinas de corrientes, y son de gran interés para el desarrollo de esta tesis, así como el estudio mediante modelos matemáticos o experimentales del fenómeno de cavitación.

A continuación, se resume los aspectos y conceptos más importantes de las mencionadas técnicas de cálculo y de la cavitación que se manejan en los artículos y documentos de reciente publicación en esta materia.

\section{Modelo de disco actuador}

En muchos documentos donde se analizan o se describen las técnicas o modelos de cálculo en el ámbito de las turbinas marinas, se empieza por el modelo de disco actuador ("Actuator Disk Model" - ADM), aunque la técnica de cálculo de modelo de disco actuador [12] [13] no es exclusiva de las turbinas de corrientes marinas, es perfectamente aplicable a éstas, y tiene gran importancia debido a su claridad conceptual.

En este caso la turbina o disco actuador es una entidad matemática que sustituye al rotor de la turbina de forma que cuando el fluido lo atraviesa, se puede evaluar la cantidad de energía extraída por la turbina.

Para tratar de representar los fenómenos físicos que se producen en las partículas fluidas a la entrada y a la salida del disco, se considera el disco como una discontinuidad, de la presión y la velocidad.

En la Figura 8, se muestra la distribución de presiones y velocidades utilizada en el modelo de disco actuador, y como se puede observar en ella, la velocidad de la corriente disminuye ligeramente a medida que se acerca a la zona de influencia del rotor, y esto se cuantifica con la ecuación [14],

$$
U\left(1-s_{1}\right)
$$


El parámetro $s_{1}$, se denomina como factor de inducción axial y en muchas publicaciones se utiliza la letra a para denotarlo [14].

Esta técnica, como se ha comentado, es de gran importancia desde el punto de vista conceptual de cara a empezar a comprender las ideas utilizadas en los diversos modelos matemáticos que se emplean en el estudio de las turbinas marinas de eje horizontal.

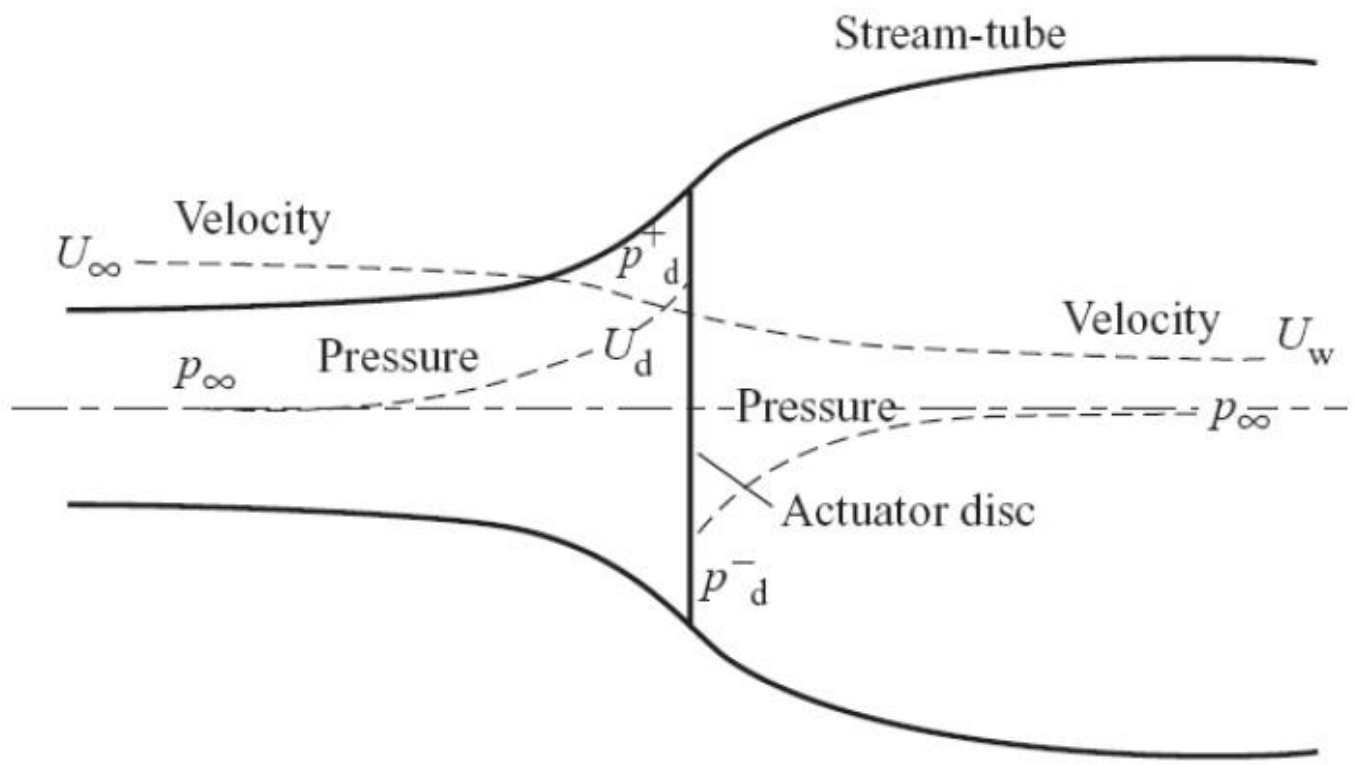

Figura 8. Perfil de presiones y velocidades axiales en función de la distancia al disco actuador [13]

La energía cinética de la corriente fluida que produce la rotación del rotor y que entra en el volumen de control del disco actuador depende de la velocidad de la corriente y del área total del rotor, de acuerdo a la siguiente ecuación [15],

$$
\frac{1}{2} \rho A U^{3}
$$

Esta ecuación es básica para poder realizar cualquier cálculo del rendimiento, y se dedujo también en el apartado 2.1 de esta tesis.

La relación entre la energía que aporta la corriente fluida y la energía cinética de rotación del rotor, constituye el coeficiente de potencia $c_{P}$, que varía con la velocidad de la corriente y la velocidad de rotación del rotor. Con el objeto, de unificar estas dos velocidades y facilitar así criterios comparativos de funcionamiento de la turbina en diferentes condiciones, se utiliza a menudo la velocidad TSR, que depende de las velocidades mencionadas y del radio del extremo de pala [16],

$$
T S R=\frac{\omega b}{U}
$$


Si la turbina a estudiar se supone que no está situada en mar abierto [17], si en un río o en una determinada zona geográfica en la que se pueda considerar que existan limitaciones para el flujo de la corriente fluida, la influencia de estos efectos, así como el de las olas [18] se debe tener en cuenta para su correcto funcionamiento.

Teoría de elementos de pala ("Blade Element Theory" - BET). Teoría del Momento de los elementos de pala ("Blade Element-Momentum Theory-BEMT)

Dividiendo la pala en un número de secciones determinado [14] [19] [20], se puede estudiar la influencia de la geometría de cada uno de los elementos de pala por separado y luego sumar sus aportaciones al funcionamiento global del rotor, esta es la base de las técnicas de elementos de pala (BET y BEMT), y es la solución más clásica para abordar el problema de calcular las cargas que se producen en la pala de una turbina eólica o de corrientes marinas.

La Teoría de elementos de pala, se basa en dos ideas clave [14]:

- No hay ninguna interacción entre los distintos elementos de pala.

- Las fuerzas en los elementos de pala solamente se determinan con los coeficientes de sustentación y arrastre.

Hay que tener en cuenta que, en el ámbito de las turbinas de corrientes marinas, existen algunos aspectos y/o fenómenos a tener en cuenta que no se producen para las turbinas eólicas, como son: La cavitación [21], influencia de la superficie libre [18], instalación de tobera, etc.

Hasta este momento se ha recopilado información acerca del método de disco actuador y del método de elementos de pala (BET y BEMT) [13], que de las técnicas o modelos existentes son las más apropiadas para llevar a cabo un método de cálculo destinado para la fase de diseño básico [22] o conceptual de una turbina de corrientes.

\section{Líneas sustentadoras y CFD}

Como se introducido en esta tesis, las técnicas de cálculo que se implementan en la fase de diseño preliminar o básico juegan un papel muy importante en el proceso productivo de turbinas, aunque está claro que, para fases más avanzadas de diseño son necesarias otras herramientas de cálculo que ofrezcan mayor precisión de resultados, y es en este momento, donde cabe citar la Teoría de Líneas sustentadoras (Lifting Line Theory - LLT) [23], y por otro lado la implementación computacional de fluidos ("Computational Fluid Dynamics" - CFD)[24].

La implementación tanto de la LLT como de los CFD, requiere un conocimiento muy extenso y el manejo de herramientas de cálculo complejas, sin embargo son esenciales para poder diseñar estructuras de pala que sean efectivas para el funcionamiento de las turbinas de corrientes.

La Teoría de Líneas Sustentadoras consiste, muy resumidamente, en suponer la pala del rotor como una línea en la que confluyen líneas de vórtice de distinta intensidad de circulación [9]. Es un modelo que es comúnmente utilizado para el cálculo de alas de aeroplanos así como para propulsores de 
buques, aunque ya empieza a haber trabajos en los que se aplica este modelo a turbinas de corrientes marinas [23].

La técnica de los CFD [25] es consiste en modelar tanto la superficies de la turbina como el fluido mediante elementos matemáticos a los que se asignan ciertas propiedades que simulen el comportamiento de cada una de las partes que componen el problema. Los modelos realizados con esta técnica son íntegramente tridimensionales $[26,27,28]$ y los resultados obtenidos son los más precisos de todas las técnicas de cálculo expuestas.

Los CFD se han ido utilizando cada más en todos los ámbitos de las ciencias y la ingeniería a medida que los equipos informáticos han mejorado en cuanto a prestaciones de cálculo, precio y tamaño.

\section{Efecto de la Cavitación [21]}

El estudio del fenómeno de cavitación en la turbinas de corrientes marinas ha originado gran cantidad de artículos y documentos. Se trata de un fenómeno que es extremadamente complejo de modelizar matemáticamente, a no ser que se utilicen criterios muy conservadores, y que ocasiona numerosos problemas en el funcionamiento de los dispositivos de aprovechamiento energético, por lo que tiene una importancia vital.

La cavitación, básicamente, consiste en que, en ciertas partes del fluido en movimiento la velocidad de las partículas sea tal que se produzca un decrecimiento de la presión por debajo de la presión de vapor, provocando la aparición de burbujas. Estas burbujas golpean las superficies del rotor afectando a su funcionamiento normal y ocasionado daños que pueden ser de gravedad.

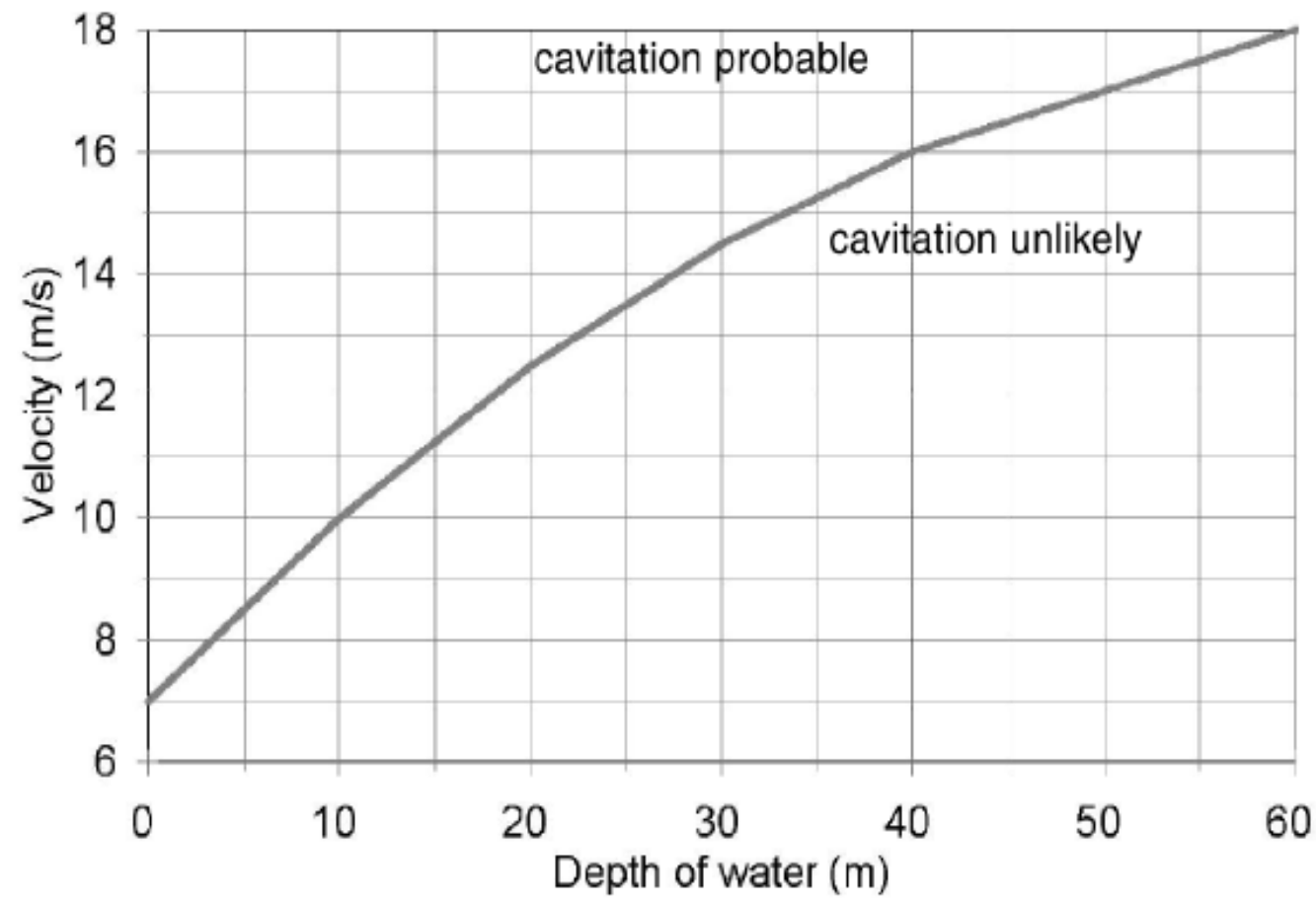

Figura 9. Relación entre velocidad del fluido y profundidad que puede producir cavitación [15] 
En el estudio de la cavitación se utiliza lo que se denomina número de cavitación, denotado por la letra griega $\sigma$ y cuya ecuación es [21],

$$
\sigma=\frac{P_{0}+\rho g h-P_{v}[T]}{\frac{1}{2} \rho v^{2}}
$$

Puesto que la cavitación se produce cuando la velocidad del fluido hace que la presión decrezca por debajo de la presión de vapor, un parámetro que reduce el efecto de la cavitación es la profundidad a la que está instalado el rotor. En la Figura 9 se muestra la relación entre velocidad del fluido, profundidad y la posibilidad de que se produzca cavitación.

Así pues, los fenómenos de la mecánica de fluidos a evitar durante el funcionamiento, son principalmente como se ha expuesto, los desprendimientos de flujo o flujos turbulentos en el entorno de las secciones de pala y la aparición de burbujas de vapor que pueden impactar con las superficies del rotor o la cavitación.

También habría que tener en cuenta la posible formación de hielo en algunas partes de la turbina, ya que, en zonas donde la velocidad del fluido sea alta, se puede producir un descenso de la presión colindante y dar lugar a acumulaciones de hielo que pueden quedar adheridas al rotor.

\subsection{VENTAJAS E INCONVENIENTES DE LOS PROCEDIMIENTOS DE CÁLCULO EXISTENTES}

El funcionamiento de una turbina de corrientes marinas es análogo al de las turbinas eólicas, es decir, están inmersas en un fluido en movimiento, que en este caso es agua de mar. El movimiento de este fluido genera unas fuerzas o cargas en las palas del rotor que hace que éste gire arrastrando consigo un generador eléctrico.

Estas cargas hidrodinámicas, se pueden calcular por distintos métodos, como se ha visto en el apartado anterior, que según su naturaleza, se podrán englobar en alguno de los grupos siguientes:

- Técnicas basadas en elementos de pala (BET y BEMT) [20].

- Disco Actuador [29]

- Líneas Sustentadoras

- Implementación computacional de fluidos (CFD) [28]

- Técnicas combinadas [30][31]

Cada uno de estos métodos de cálculo tiene sus ventajas e inconvenientes, que se exponen a continuación:

\section{Método del disco actuador ("Actuator Disk Model" - ADM)}

Como se ha explicado anteriormente, en los artículos que utilizan este procedimiento de cálculo, el rotor se modeliza matemáticamente mediante un disco permeable [12][13] a la corriente fluida en el que se supone que se va a producir una discontinuidad en la presión y velocidades del flujo (ver 
Figura 8), es decir, cuando una partícula fluida atraviesa el rotor se considera que no conserva la línea de corriente por la que seguía su trayectoria hasta acercarse al rotor.

Por tanto en este caso el Teorema de Bernoulli no sería aplicable, solamente podría serlo para una partícula desde el aguas arriba del rotor hasta las inmediaciones de éste justo a la entrada [12]. Lo mismo ocurre para las partículas que abandonan el rotor la línea de corriente se forma desde justo a la salida del rotor hacia aguas abajo.

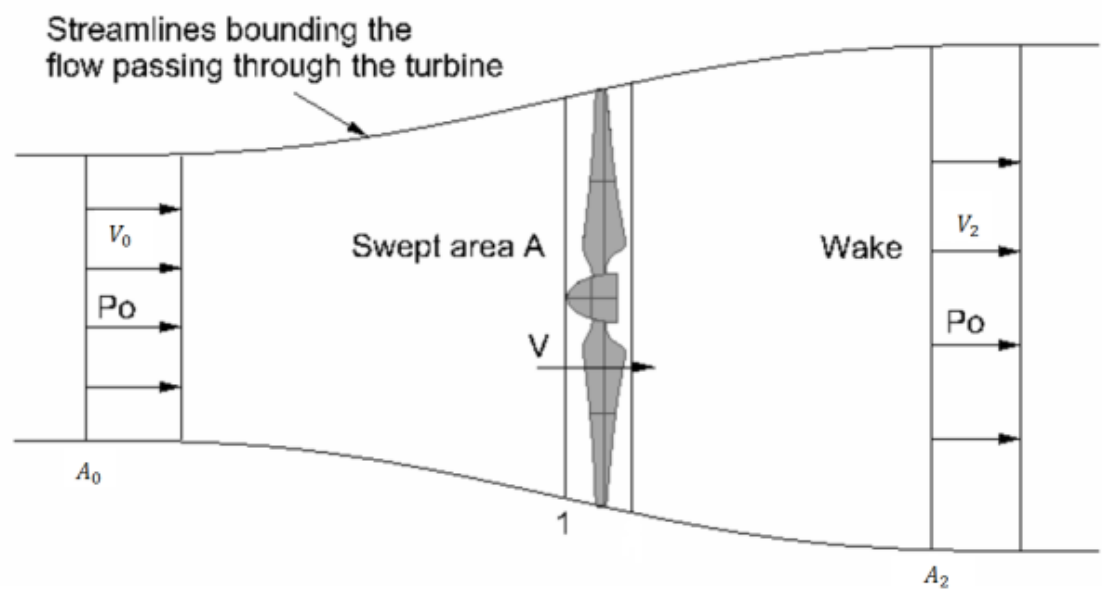

Figura 10. Representación clásica de un modelo de disco actuador [24]

Uno de los mayores inconvenientes de este método de cálculo es que presenta algunas inconsistencias desde el punto de vista teórico, ya que se suelen utilizar artificios para representar matemáticamente ciertos parámetros del modelo como el salto de presiones y velocidades a la entrada y salida del rotor.

Otra inconsistencia que existe, y que es común a todas las publicaciones relacionadas con esta metodología es que siempre se considera que las líneas fluidas se expanden al atravesar el rotor [31, 32]. Este concepto es utilizado de forma universal, sin embargo, en prácticamente ningún artículo se explica la razón de esta expansión de las líneas fluidas.

Está claro que si se quiere obtener la energía por unidad de tiempo extraída por el rotor, con arreglo al esquema representado en la Figura 10, primero se aplica la conservación de la masa entre los puntos 0 , situado aguas abajo del rotor y 2 , situado aguas arriba. Para ello se aplica la ecuación,

$$
\rho A_{0} V_{0}=\rho A_{2} V_{2} \rightarrow A_{0} V_{0}=A_{2} V_{2}
$$

Y ahora, mediante la ecuación de la energía se obtiene la energía por unidad de tiempo que se extraería en el rotor,

$$
P=\frac{1}{2} \rho\left(A_{2} V_{2}^{3}-A_{0} V_{0}^{3}\right)
$$


En estas ecuaciones se puede observar que si no se consideran distintas las áreas $\mathrm{A}_{0}$ y $\mathrm{A}_{2}$, la potencia extraída se cancelaría, por esto se utiliza la expansión de las líneas fluidas a la salida del rotor, aunque aparentemente no tenga una base teórica.

Sin embargo, posiblemente esto ocurra porque se utilizan simplificaciones de las ecuaciones de continuidad y energía, también debido a la consideración de que el sistema es estacionario, cuando en realidad la naturaleza de problema está más cercana a fenómenos transitorios.

Si se plantea, un modelo de disco actuador en el que el área del volumen de control aguas abajo sea igual al área del rotor y al área de la superficie de control aguas arriba e igual a la variable $\mathrm{A}$. Se pueden obtener ecuaciones que tiene sentido físico, por ejemplo, si se acude a la expresión general de la ecuación de continuidad para un fluido de densidad constante $\rho$, esta sería.

$$
\int_{V C} \bar{v} \overline{d s}=0
$$

Y aplicada al modelo de disco actuador, suponiendo una simetría radial con respecto al eje del rotor y que la corriente fluida se desplaza a la velocidad $U$, queda,

$$
\int_{0} U d s=U A=2 \pi \int_{2} v(r) d r
$$

Es decir, a la salida del rotor existirá una distribución radial de velocidades del fluido que no se puede suponer como constante, ya que si no se caería en la contradicción explicada más arriba. Si se hace el mismo razonamiento para obtener el empuje $T$ que el fluido ejerce sobre el rotor,

$$
T=2 \pi \rho \int_{2} v(r)^{2} d r-A \rho U^{2}
$$

Y lo mismo para la energía por unidad de tiempo extraída por el rotor,

$$
P=\pi \rho \int_{2} v(r)^{3} d r-\frac{1}{2} A \rho U^{3}
$$

Por tanto, para resolver el problema hay que tratar de obtener la distribución de velocidades v(r), y no suponerla como constante, ya que si no hay realizar un artificio como el de suponer que la línea fluida se expande atravesar el rotor. Esto no quiere decir que obtener v(r) sea algo sencillo, pero sí es la forma de plantear un modelo de disco actuador de forma coherente.

Otro inconveniente importante de los modelos matemáticos basados en disco actuador, es que no resulta fácil relacionar las ecuaciones planteadas con la geometría del pala del rotor. Hay que notar que las ecuaciones están planteadas de forma general para el rotor, pero no para poder dilucidar como afectarían variaciones de la geometría de cada pala a la potencia extraída y al empuje que la corriente fluida ejerce sobre la turbina. 


\section{Líneas sustentadoras (LLT)}

Esta teoría, como se comentó anteriormente, se fundamenta en que cada elemento de pala se modeliza mediante una "línea sustentadora". Y es aquí donde empieza el gran inconveniente de esta metodología, y que se sustenta en ideas muy poco intuitivas o de difícil comprensión.

Solamente los lectores dedicados a la investigación y utilización de estos conceptos son capaces de resolver un problema mediante esta técnica, que no está exenta de ciertos artificios no fundamentados al igual que ocurre con los modelos basados en disco actuador.

\section{Elementos finitos (CFD)}

Quizás esta sea la metodología más adecuada para el problema de conocer los parámetros de funcionamiento de un problema complejo como el de una turbina de corrientes marina [33]. Se pueden definir geometrías complejas y obtener cómo afectan pequeñas variaciones de ésta a la mejora del rendimiento de la turbina, se pueden modelizar los fenómenos de turbulencia, interacción entre distintas partes del dispositivo, etc.

Sin embargo, el gran inconveniente es que requiere de la utilización de software avanzado de gran coste económico, de recursos informáticos que se traduce en elevado tiempo de cálculo y trabajo de elaboración del modelo.

En la siguiente tabla se muestra un resumen de las ventajas e inconvenientes de cada uno de los métodos de cálculo utilizados en el ámbito de las turbinas de corrientes marinas.

\begin{tabular}{|c|c|c|c|c|}
\hline & $\begin{array}{l}\text { Modelos de Disco } \\
\text { Actuador (ADM) }\end{array}$ & $\begin{array}{c}\text { Teoría de Líneas } \\
\text { Sustentadoras (LLT) }\end{array}$ & $\begin{array}{l}\text { Modelos basados } \\
\text { en elementos } \\
\text { finitos (CFD) }\end{array}$ & $\begin{array}{c}\text { Técnicas basadas en } \\
\text { elementos de pala } \\
\text { (BET, BEMT) }\end{array}$ \\
\hline Ventajas & Conceptualmente intuitivo & $\begin{array}{l}\text { Es uno de los métodos } \\
\text { más utilizados }\end{array}$ & $\begin{array}{l}\text { Gran precisión de } \\
\text { resultados } \\
\text { Se puede elaborar } \\
\text { un modelo de rotor } \\
\text { con gran detalle } \\
\text { Método } \\
\text { recomendado para } \\
\text { el diseño de una } \\
\text { turbina de eje } \\
\text { horizontal }\end{array}$ & $\begin{array}{l}\text { Es la técnica más } \\
\text { comúnmente utilizada } \\
\text { Sencillez de cálculos y } \\
\text { de conceptos } \\
\text { Puede servir como } \\
\text { introducción antes de } \\
\text { manejar otro } \\
\text { procedimiento de } \\
\text { cálculo más complejo } \\
\begin{array}{l}\text { Se puede tener una idea } \\
\text { rápida de los resultados } \\
\text { de un determinado rotor }\end{array}\end{array}$ \\
\hline
\end{tabular}




\begin{tabular}{|c|c|c|c|c|}
\hline & $\begin{array}{l}\text { Modelos de Disco } \\
\text { Actuador (ADM) }\end{array}$ & $\begin{array}{c}\text { Teoría de Líneas } \\
\text { Sustentadoras (LLT) }\end{array}$ & $\begin{array}{l}\text { Modelos basados } \\
\text { en elementos } \\
\text { finitos (CFD) }\end{array}$ & $\begin{array}{c}\text { Técnicas basadas en } \\
\text { elementos de pala } \\
\text { (BET, BEMT) }\end{array}$ \\
\hline Inconvenientes & $\begin{array}{l}\text { Gran dificultad en relacionar } \\
\text { una determinada geometría } \\
\text { de rotor con un modelo } \\
\text { Se suelen utilizar artificios } \\
\text { matemáticos para paliar } \\
\text { posibles incongruencias en } \\
\text { las variables que intervienen } \\
\text { el problema }\end{array}$ & $\begin{array}{l}\text { Este método implica la } \\
\text { ejecución de cálculos } \\
\text { complejos, haciendo } \\
\text { difícil la obtención de } \\
\text { resultados de forma } \\
\text { rápida. } \\
\text { Artificio conceptual para } \\
\text { hacer cumplir el } 2^{\circ} \\
\text { Teorema de Helmotz }\end{array}$ & $\begin{array}{lr}\begin{array}{l}\text { Requiere } \\
\text { trabajo mucho } \\
\text { conocimiento } \\
\text { confeccionar } \\
\text { modelo }\end{array} \\
\begin{array}{l}\text { Gran consumo de } \\
\text { recursos de cálculo }\end{array} \\
\begin{array}{lr}\text { Se necesitan } \\
\text { programas }\end{array} \\
\begin{array}{lr}\text { costosos muy } \\
\text { confeccionar } \\
\text { modelos }\end{array}\end{array}$ & $\begin{array}{l}\text { Tiene que contrastarse } \\
\text { con CFD o LLT } \\
\text { No se tiene en cuenta } \\
\text { fenómenos de } \\
\text { turbulencia debido a su } \\
\text { simplificaciones } \\
\text { No se tienen en cuenta } \\
\text { interacciones } \\
\text { hidrodinámicas entre los } \\
\text { distintos elementos de } \\
\text { pala }\end{array}$ \\
\hline
\end{tabular}

Tabla 2. Tabla comparativa de los métodos de cálculo utilizados en el ámbito de las turbinas de corrientes marinas 


\section{PROCEDIMIENTO ALTERNATIVO DE CÁLCULO}

Como se ha expuesto en los apartados anteriores, los métodos o herramientas de cálculo clásicas basadas en el estudio de los elementos de pala (BET, BEMT), siguen estando de plena actualidad, y esto se comprueba en los numerosos artículos dedicados a las turbinas de corrientes marinas, que incluyen su estudio.

Estas técnicas, por su sencillez de manejo, se siguen utilizando tanto para el aprendizaje como para los procesos industriales, puesto que se permite llevar a cabo análisis cualitativos y cálculos preliminares de forma rápida.

Y es en este ámbito, donde se propone una mejora. Las técnicas de elementos de pala como su propio nombre indica, se basan en analizar cada elemento de pala por separado y luego sumar su contribución global al rotor, pero no deja de ser una técnica bidimensional.

Es decir, si se calculan los parámetros de una determinada sección de pala, estos permanecen invariables en el caso de que se modifique cualquier otra sección de pala. $Y$ es en este sentido, donde se quiere realizar una aportación para tratar de mejorar los modelos basados en elementos de pala, de forma que cada elemento de pala interactúe con el resto de elementos de la pala, y por tanto, se busca una relación tridimensional.

\subsection{FUNDAMENTOS MATEMÁTICOS}

La idea básica en que se sustenta este procedimiento, parte de la observación de algunas fotografías de la estela [34] que se forma en el aire detrás de los aeroplanos [9]. En ellas se puede observar el campo de velocidades que se genera aguas abajo, que consiste en dos vórtices, cuya vorticidad en cada uno de ellos es igual y opuesta, el modelo matemático confeccionado consiste en este concepto.

Es decir, si se secciona el campo de velocidades del fluido por un plano que esté situado justo detrás de ala, se obtendrá un campo de velocidades que está compuesto por dos vórtices con intensidades de circulación iguales en módulo y opuestas en sentido de giro.

Por tanto, la hipótesis básica del modelo es que esta estela compuesta por dos vórtices contrarrotativos es extrapolable igualmente a un sistema giratorio, como es la pala de una turbina de corrientes.

Con el objeto de fundamentar lo máximo posible esta hipótesis, se han buscado documentos que modelicen el flujo fluido en una turbina de corrientes [35, 36, 37, 38] en los que puedan encontrarse pruebas de la presencia de estos vórtices.

En este sentido, en primer lugar, se analiza el flujo representado en la Figura 11. En ella se establece una categoría de colores para las zonas con distinta vorticidad, y se puede ver que la pala está inmersa en una zona de vorticidad equivalente a la partes del fluido que abandonan la pala tanto en la zona del núcleo de pala como del extremo (color verde oscuro). 


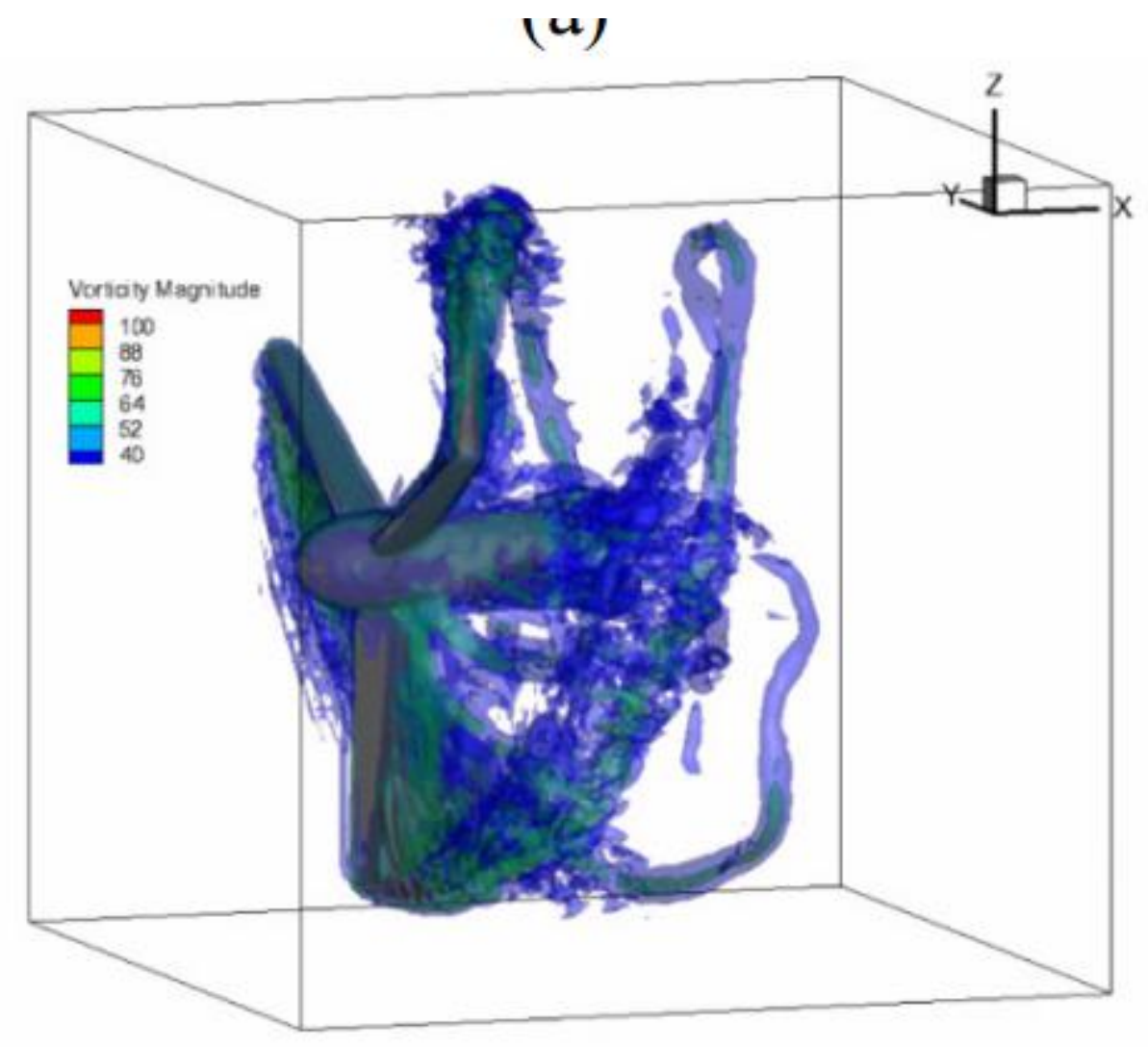

Figura 11. Modelado 3D de un flujo creado por la técnica de CFD [36]

Esta zona de vorticidad puede representar una línea de vórtice que atraviesa la pala y la abandona tanto por la zona del núcleo de pala como por el extremo, y parece que esta observación confirma el Teorema de Helmolt [39] para vórtices [9], que establece que la intensidad de la circulación a lo largo de una línea de vórtice se mantiene constante.

Si esto es así, el campo de velocidades originado al seccionar el flujo fluido por un plano situado en la estela de pala, estaría formado por dos vórtices contrarrotativos.

En otra publicación [37], se ha modelizado el flujo fluido de varias turbinas marinas mediante mediante la técnica de CFD, y si se observan las zonas de núcleo y extremo de pala se ve claramente la presencia de la citada línea de vórtice y como esta abandona la pala en estos puntos (Figura 12).

Este concepto también se puede observar, aunque con menos claridad, que en las anteriores en la Figura 13.

Puesto que se trata de un concepto nuevo, puede existir la controversia, de si esta misma estela (con dos vórtices contrarrotativos) se produce igualmente en un movimiento giratorio, pero en este sentido, y aparte de los indicios encontrados en publicaciones, cabe argumentar lo siguiente: Parece obvio que si el aeroplano efectúa un giro, estos mismos vórtices seguirán existiendo con la misma naturaleza que en el movimiento lineal, por lo que la idea sigue teniendo validez. No parece lógico pensar que si existían durante el movimiento lineal, desaparezcan automáticamente o el flujo cambie drásticamente si se produce un movimiento giratorio. 


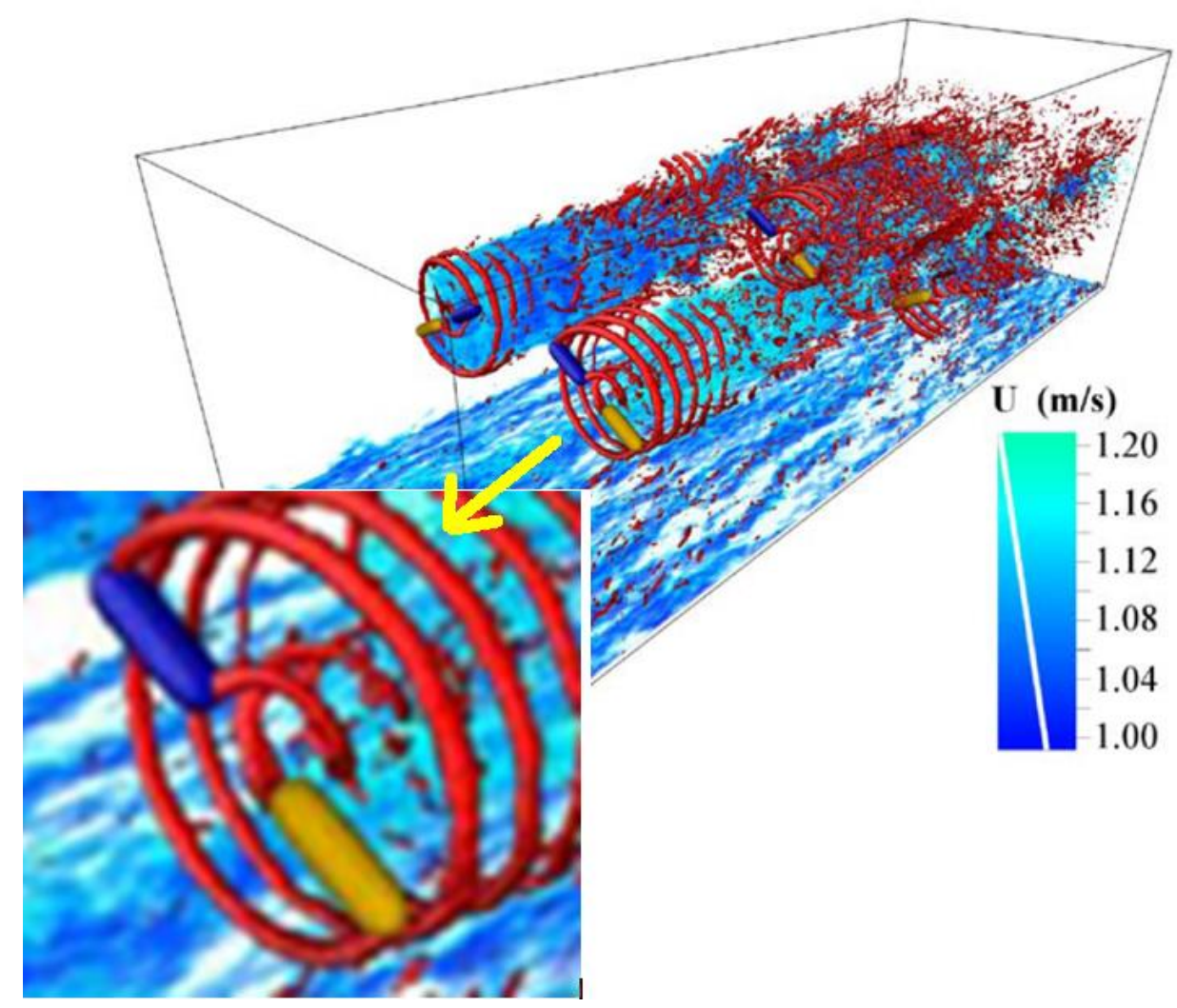

Figura 12. Modelado 3D de un flujo creado por la técnica de CFD [37]

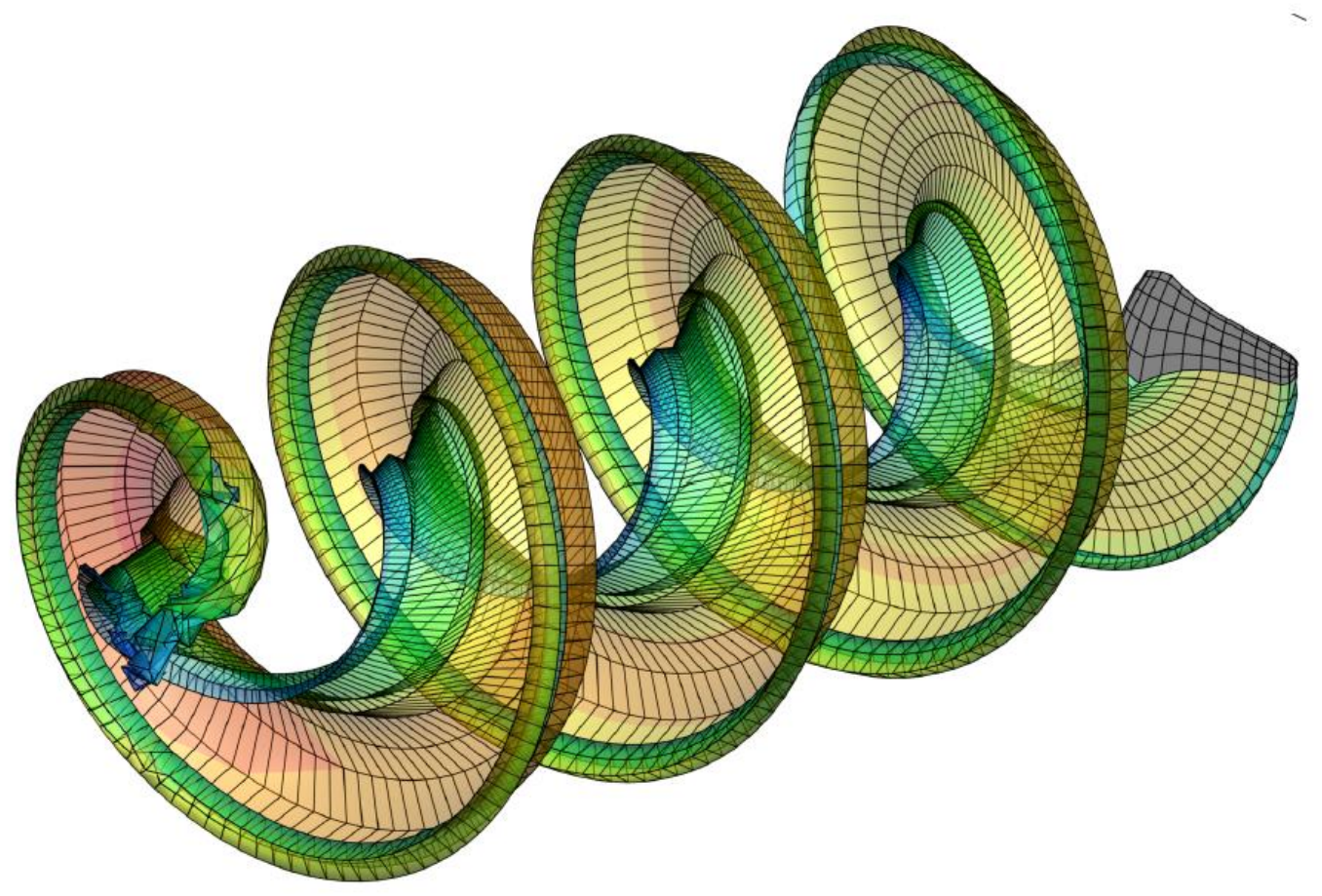

Figura 13. Modelado 3D de un flujo creado por la técnica de CFD [35] 
Para plantear este modelo de la forma más sencilla posible, hay que hacer uso, por un lado, de la técnica físico-matemática del volumen de control [40], que permite obtener resultados a partir del comportamiento del fluido en sus fronteras, una vez que éstas han sido definidas, dando un enfoque del problema lo más cercano posible a la mecánica clásica y conceptualmente intuitivo.

En la fase final del desarrollo matemático, para poder completar la obtención de resultados, será necesario combinar las ecuaciones obtenidas mediante el volumen de control con la teoría bidimensional de elementos de pala (BET) [41].

Si se define un rotor en el que el radio del núcleo es a , el radio total es b y el número de palas es Z; entonces un volumen de control que contenga la pala en estudio podría ser el que se representa en la Figura 14 (hay que tener en cuenta que se podría definir este volumen de control de muchas formas siendo todas válidas, sin embargo se ha elegido esta por comodidad a la hora de las operaciones).

Con esto, se puede empezar el desarrollo matemático. Por tanto, el volumen de control elegido, está formado por los vértices $A B C D E F G H$ y su longitud es igual a la longitud de la pala en estudio $b-a$ y tiene una sección cuadrada de lado $\frac{2 \pi \mathrm{a}}{\mathrm{Z}}$.

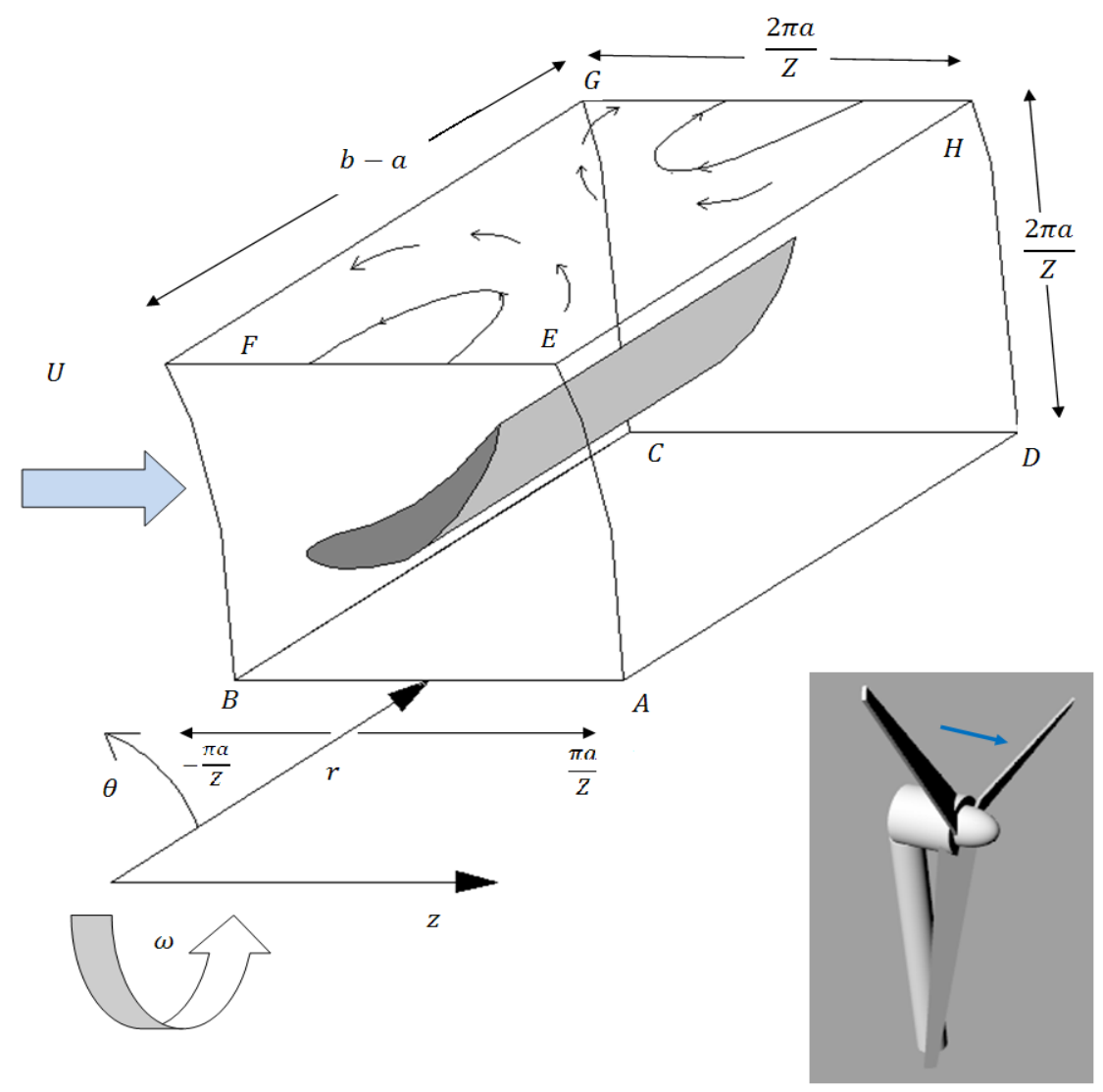

Figura 14. Esquema principal del planteamiento del problema 
Para terminar de plantear el problema, es necesario establecer unas distribuciones de velocidades en las caras de salida del fluido del volumen de control establecido, que son las caras EFGH y ADEH. En las caras ABFE y

En la Figura 15, se muestra el campo de velocidades del fluido en un plano determinado por los vértices $E F G H$, que está situado justo detrás de la pala, con los vórtices $V_{1}$ y $V_{2}$. Este plano estaría definido por los puntos EFGH, y las ecuaciones del campo de velocidades son:

$$
\begin{gathered}
v_{r}=\frac{\Gamma}{2 \pi}\left[\frac{z}{(r-a)^{2}+z^{2}}-\frac{z}{(r-b)^{2}+z^{2}}\right] \\
v_{z}=\frac{\Gamma}{2 \pi}\left[-\frac{r-a}{(r-a)^{2}+z^{2}}+\frac{r-b}{(r-b)^{2}+z^{2}}\right]
\end{gathered}
$$

Estas dos ecuaciones del campo de velocidades descrito constituyen la base matemática del procedimiento de cálculos, como se verá más adelante.

En el plano CDGH se supone que el fluido circula paralelamente a éstas y no las atraviesa en ningún momento. En la cara EFGH la velocidad con la que el fluido atraviesa la cara corresponderá a una distribución que se denota como $\mathrm{v}_{\omega \mathrm{r}}^{*}$. Análogamente en la cara ADEH esta distribución de velocidades se denota como $\mathrm{U}^{*}[42]$, tal y como se representan en la Figura 16. Estas dos distribuciones son desconocidas a priori y se supone que solamente dependen de la variable radial $r$.

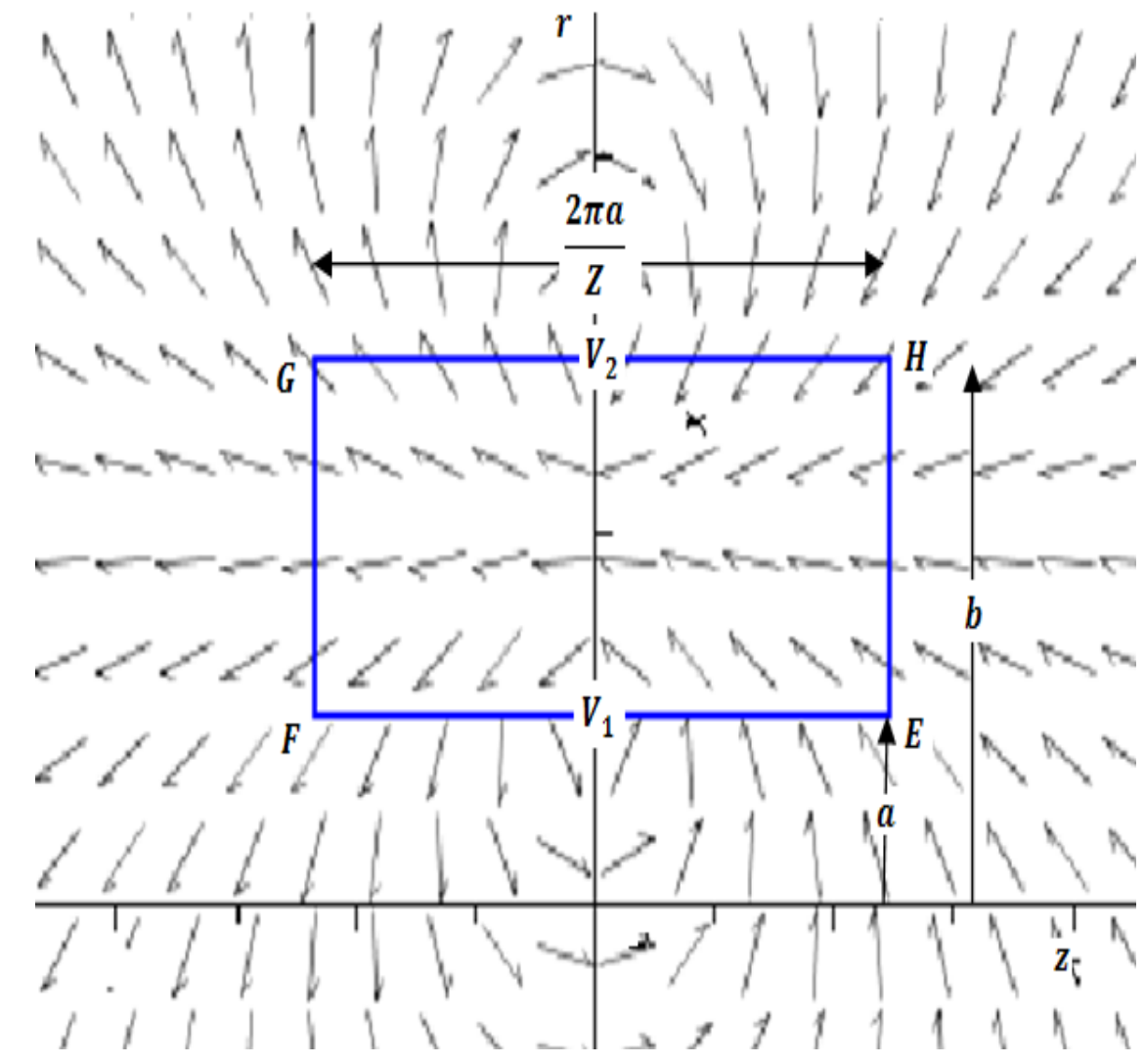

Figura 15. Campo de velocidades originado por la pala a su paso 


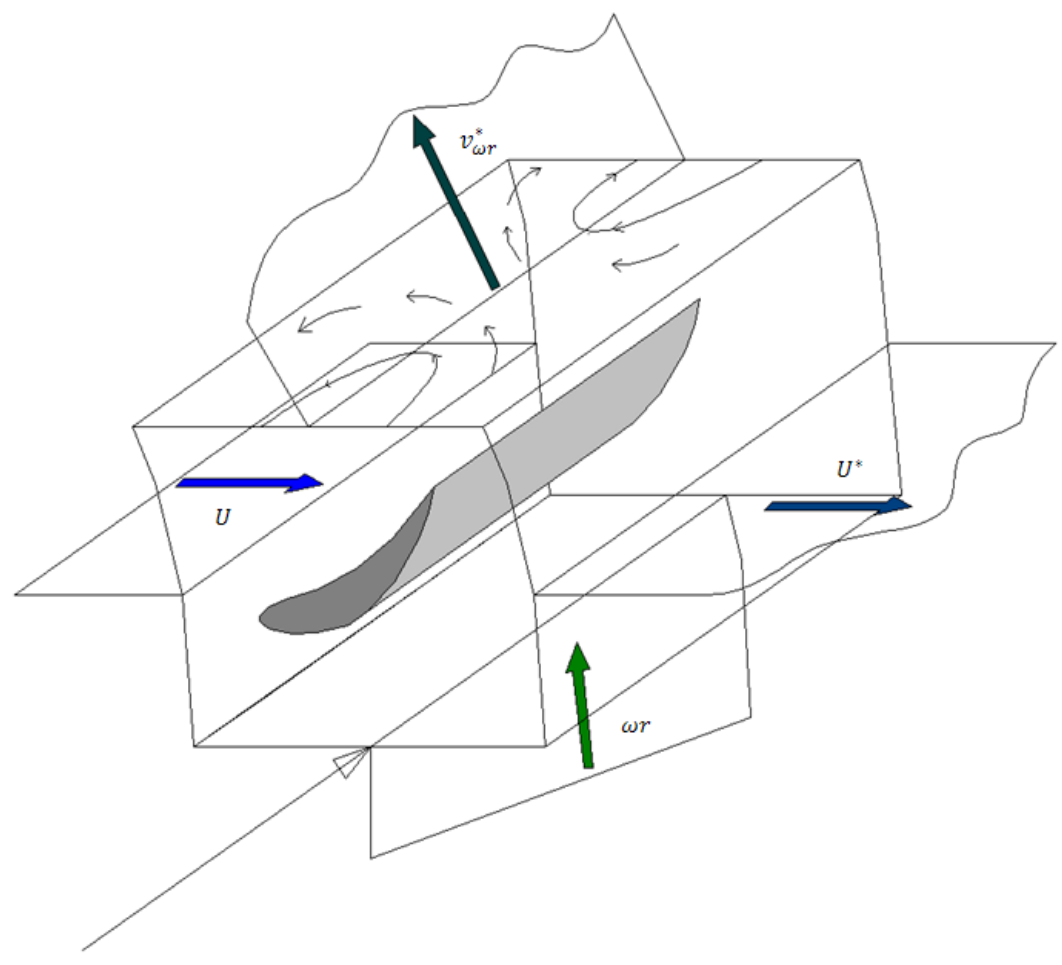

Figura 16. Esquema de las velocidades que interviene en el problema

En los siguientes apartados, se estudiará el volumen de control definido en la Figura 14, para ello, se calculará el balance masa a través de las superficies-frontera del mismo, así como el balance de momento lineal y de energía, considerando siempre que el sistema en estudio está en régimen estacionario. De las ecuaciones deducidas se podrán obtener las fuerzas que actúan en la pala, la potencia total que se extrae del rotor y el rendimiento de éste.

\subsubsection{BALANCE DE MASA}

Para calcular el flujo de masa que interviene en el sistema, hay que considerar la masa que atraviesa cada una de las superficies frontera del volumen de control, y puesto que en el interior de éste no se crea masa y que éste está en régimen estacionario, implica que el balance másico global es nulo, esto es,

$$
\int_{S C} \rho(\bar{v} \overline{d s})=\int_{A B C D} \rho(\bar{v} \overline{d s})+\int_{E F G H} \rho(\bar{v} \overline{d s})+\int_{B C F G} \rho(\bar{v} \overline{d s})+\int_{A D E H} \rho(\bar{v} \overline{d s})+\int_{A B F E} \rho(\bar{v} \overline{d s})+\int_{C D G H} \rho(\bar{v} \overline{d s})=0
$$

En general, la velocidad del fluido que entra en el volumen de control, es conocida, viene dada por la velocidad de la corriente $\mathrm{U}$ (cara $\mathrm{BCFG}$ ) y por la velocidad de rotación $\omega$ (cara $A B C D$ ).

No ocurre lo mismo para la velocidad de las partículas que salen, y para ello se utilizarán las incógnitas $U^{*} y v_{\omega r}^{*}$, definidas en el apartado anterior, que se suponen no dependen de $\mathrm{z} y \theta$. De este modo, desglosando cada uno de los términos de la ecuación Ec. 29, 


$$
\begin{array}{rr}
\int_{A B C D} \rho(\bar{v} \overline{d s})=-\rho \omega \int_{A B C D} r d r d z & E c .30 \\
\int_{B C F G} \rho(\bar{v} \overline{d s})=-\rho U \int_{B C F G} d r(r d \theta) & E c .31 \\
\int_{E F G H}^{\rho} \rho(\bar{v} \overline{d s})=\rho \int_{E F G H} v_{\omega r}^{*} d r d z & E c .32 \\
\int_{A D E H}^{\rho} \rho(\bar{v} \overline{d s})=\rho \int_{A D E H} U^{*} d r(r d \theta) & E c .33 \\
\int_{A B F E} \rho(\bar{v} \overline{d s}) \cong 0 & E c .34 \\
\int_{C D G H} \rho(\bar{v} \overline{d s}) \cong 0 & E c .35
\end{array}
$$

Y ahora sustituyendo cada término en la ecuación Ec. 29,

$$
\int_{V C} \rho(\bar{v} \overline{d s})=-\rho \omega \int_{A B C D} r d r d z+\rho \int_{E F G H} v_{\omega r}^{*} d r d z-\rho U \int_{B C F G} d r(r d \theta)+\rho \int_{A D E H} U^{*} d r(r d \theta) \quad \text { Ec. } 36
$$

Como se ve en la Figura 14, los límites de integración de las superficies $A B C D$ y EFGH son iguales, lo mismo ocurre para BCFG y ADEH, así la ecuación Ec. 36 queda,

$$
\int_{A B C D}\left(v_{\omega r}^{*}-\omega r\right) d r d z+\int_{A D E H}\left(U^{*}-U\right) d r(r d \theta)=0
$$

Integrando, se obtiene,

$$
\int_{a}^{b}\left(v_{\omega r}^{*}-\omega r\right) d r \int_{-\frac{\pi a}{Z}}^{\frac{\pi a}{Z}} d z+\int_{a}^{b}\left(U^{*}-U\right) d r \int_{0}^{\theta} r d \theta=\frac{2 \pi a}{Z} \int_{a}^{b}\left(v_{\omega r}^{*}-\omega r\right) d r+\frac{2 \pi a}{Z} \int_{a}^{b}\left(U^{*}-U\right) d r=0 \rightarrow \quad \text { Ec. } 38
$$

Según las dimensiones del volumen de control elegido, $\int_{0}^{\theta} \operatorname{rd} \theta=\frac{2 \pi a}{\mathrm{Z}}$, entonces se obtiene la expresión,

$$
\frac{2 \pi a}{Z} \int_{a}^{b}\left(v_{\omega r}^{*}-\omega r+U^{*}-U\right) d r=0 \rightarrow \int_{a}^{b}\left(v_{\omega r}^{*}-\omega r+U^{*}-U\right) d r=0
$$

\subsubsection{BALANCE DE MOMENTO LINEAL}

En este apartado, análogamente al anterior, se calculará el flujo de momento lineal del volumen de control en estudio, que será igual a la resultante de fuerzas exteriores que se aplican en la pala, es decir, mediante este cálculo se obtendrán las fuerzas que actúan en la base de la pala, esto es,

$$
\sum F_{\text {ext }}=\int_{S C} \rho \bar{v}(\bar{v} \bar{v})=\int_{A B C D} \rho \bar{v}(\bar{v} \overline{d s})+\int_{E F G H} \rho \bar{v}(\bar{v} \bar{v} s)+\int_{B C F G} \rho \bar{v}(\bar{v} \overline{d s})+\int_{A D E H} \rho \bar{v}(\bar{v} \overline{d s})+\int_{A B F E} \rho \bar{v}(\bar{v} \overline{d s})+\int_{C D G H} \rho \bar{v}(\bar{v} \overline{d s})
$$

Desarrollando cada uno de los sumandos de la expresión anterior, 


$$
\begin{aligned}
& \int_{A B C D} \rho \bar{v}(\bar{v} \overline{d s})=\int_{A B C D} \rho\left(\omega r \overline{u_{\theta}}\right)(-\omega r d r d z)=-\rho \omega^{2} \overline{u_{\theta}} \int_{A B C D} r^{2} d r d z \\
& \int_{E F G H} \rho \bar{v}(\bar{v} \overline{d s})=\int_{E F G H} \rho\left(v_{\omega r}^{*} \overline{u_{\theta}}+v_{r} \overline{u_{r}}+v_{z} \overline{u_{z}}\right)\left(v_{\omega r}^{*} d r d z\right)= \\
& \rho \overline{u_{\theta}} \int_{E F G H}\left(v_{\omega r}^{*} d r d z\right)+\rho \overline{u_{r}} \int_{E F G H}\left(v_{\omega r}^{*} v_{r} d r d z\right)+\rho \overline{u_{z}} \int_{E F G H}\left(v_{\omega r}^{*} v_{z} d r d z\right) \\
& \int_{B C F G} \rho \bar{v}(\bar{v} \overline{d s})=\int_{B C F G} \rho\left(U \overline{u_{z}}\right)(-U d r(r d \theta))=-\rho U^{2} \overline{u_{z}} \int_{B C F G} d r(r d \theta) \\
& \int_{A D E H} \rho \bar{v}(\bar{v} \overline{d s})=\int_{A D E H} \rho\left(U^{*} \overline{u_{z}}\right)\left(U^{*} d r(r d \theta)\right)=\rho \overline{u_{z}} \int_{A D E H}\left(U^{*}\right)^{2} d r(r d \theta)
\end{aligned}
$$

Por la geometría del problema, el flujo de masa que atraviesa las superficies definidas por los vértices ABFE y CDGH es despreciable,

$$
\begin{aligned}
& \int_{A B F E} \rho \bar{v}(\bar{v} \overline{d s}) \cong 0 \\
& \int_{C D G H} \rho \bar{v}(\bar{v} \overline{d s}) \cong 0
\end{aligned}
$$

Sustituyendo en la ecuación Ec. 40, ésta queda,

$$
\begin{gathered}
\sum F_{\text {ext }}= \\
-\rho \omega^{2} \overline{u_{\theta}} \int_{A B C D} r^{2} d r d z+\rho \overline{u_{\theta}} \int_{E F G H}\left(v_{\omega r}^{*} d r d z\right)+\rho \overline{u_{r}} \int_{E F G H}\left(v_{\omega r}^{*} v_{r} d r d z\right)+\rho \overline{u_{z}} \int_{E F G H}\left(v_{\omega r}^{*} v_{z} d r d z\right) \\
-\rho U^{2} \overline{u_{z}} \int_{B C F G} d r(r d \theta)+\rho \overline{u_{z}} \int_{A D E H}\left(U^{*}\right)^{2} d r(r d \theta)
\end{gathered}
$$

Las superficies de control $A B C D$ y $E F G H$ tienen los mismos límites de integración, lo mismo ocurre con las superficies BCFG y ADEH. Por tanto la ecuación Ec. 47 se puede escribir,

$$
\begin{gathered}
\sum F_{\text {ext }}=\rho \overline{u_{\theta}} \int_{A B C D}\left(v_{\omega r}^{*}{ }^{2}-\omega^{2} r^{2}\right) d r d z+\rho \overline{u_{r}} \int_{E F G H}\left(v_{\omega r}^{*} v_{r} d r d z\right)+\rho \overline{u_{z}} \int_{E F G H}\left(v_{\omega r}^{*} v_{z} d r d z\right)-\rho \overline{u_{z}} \int_{B C F G}\left(U^{* 2}-U^{2}\right) d r d \theta \rightarrow \\
\sum F_{e x t}=\rho \overline{u_{\theta}} \int_{a}^{b}\left(v_{\omega r}^{*}{ }^{2}-\omega^{2} r^{2}\right) d r \int_{-\frac{\pi a}{Z}}^{\frac{\pi a}{z}} d z+\rho \overline{u_{r}} \int_{a}^{b} v_{\omega r}^{*} d r \int_{-\frac{\pi a}{Z}}^{\frac{\pi a}{z}} v_{r} d z+\rho \overline{u_{z}} \int_{a}^{b} v_{\omega r}^{*} d r \int_{-\frac{\pi a}{Z}}^{\frac{\pi a}{z}} v_{z} d z \\
-\rho \overline{u_{z}} \int_{a}^{b}\left(U^{* 2}-U^{2}\right) d r \int_{0}^{\theta} r d \theta
\end{gathered}
$$

Separando la fuerza exterior en componentes y teniendo en cuenta que $\int_{-\frac{\pi a}{z}}^{\frac{\pi a}{z}} d z=\frac{2 \pi a}{z} y \int_{-\frac{\pi a}{z}}^{\frac{\pi a}{z}} r d \theta=$ $\frac{2 \pi \mathrm{a}}{\mathrm{Z}}$, éstas quedan, 


$$
\begin{gathered}
F_{\theta}=\rho \frac{2 \pi a}{Z} \int_{a}^{b}\left(v_{\omega r}^{*}-\omega^{2} r^{2}\right) d r \\
F_{r}=\rho \int_{a}^{b} v_{\omega r}^{*} d r \int_{-\frac{\pi a}{Z}}^{\frac{\pi a}{Z}} v_{r} d z=0
\end{gathered}
$$

Ya que, de acuerdo a la ecuación Ec. 27, que corresponde con la expresión de $\mathrm{v}_{\mathrm{r}}$, se observa que $\mathrm{v}_{\mathrm{r}}(-\mathrm{z})=-\mathrm{v}_{\mathrm{r}}(\mathrm{z})$, esto implica que la integral en intervalos simétricos con respecto a $\mathrm{z}$ sea nula.

$$
F_{Z}=\rho \int_{a}^{b} v_{\omega r}^{*} d r \int_{-\frac{\pi a}{Z}}^{\frac{\pi a}{Z}} v_{z} d z-\rho \frac{2 \pi a}{Z} \int_{a}^{b}\left(U^{* 2}-U^{2}\right) d r
$$

Por otro lado, sustituyendo la expresión $\mathrm{v}_{\mathrm{z}}$ de la ecuación Ec. 28,

$$
\begin{gathered}
\int_{-\frac{\pi a}{Z}}^{\frac{\pi a}{Z}} v_{Z} d z=\frac{\Gamma}{2 \pi} \int_{-\frac{\pi a}{Z}}^{\frac{\pi a}{Z}}\left[-\frac{r-a}{(r-a)^{2}+z^{2}}+\frac{r-b}{(r-b)^{2}+z^{2}}\right] d z=\frac{\Gamma}{2 \pi}\left[-\arctan \left(\frac{Z}{r-a}\right)+\arctan \left(\frac{Z}{r-b}\right)\right]_{-\frac{\pi a}{Z}}^{\frac{\pi a}{Z}} \rightarrow \\
\int_{-\frac{\pi a}{Z}}^{\frac{\pi a}{Z}} v_{Z} d z=\frac{\Gamma}{\pi}\left\{\arctan \left[\frac{\pi a}{Z(r-b)}\right]-\arctan \left[\frac{\pi a}{Z(r-a)}\right]\right\}
\end{gathered}
$$

Entonces, la componente de la fuerza exterior en la dirección z,

$$
F_{z}=-\rho \frac{\Gamma}{\pi} \int_{a}^{b} v_{\omega r}^{*}\left\{\arctan \left[\frac{\pi a}{Z(b-r)}\right]+\arctan \left[\frac{\pi a}{Z(r-a)}\right]\right\} d r-\rho \frac{2 \pi a}{Z} \int_{a}^{b}\left(U^{* 2}-U^{2}\right) d r
$$

Y si finalmente para simplificar, si se denota $\varphi(r)$ como,

$$
\varphi(r)=\arctan \left[\frac{\pi a}{Z(b-r)}\right]+\arctan \left[\frac{\pi a}{Z(r-a)}\right]
$$

Entonces la fuerza en la dirección z queda,

$$
F_{z}=-\rho \int_{a}^{b}\left[\frac{\Gamma}{\pi} v_{\omega r}^{*} \varphi(r)+\frac{2 \pi a}{Z}\left(U^{* 2}-U^{2}\right)\right] d r
$$




\subsubsection{BALANCE DE ENERGÍA}

Por último que por determinar el flujo energía del volumen de control en estudio. En este caso, éste queda determinado por la ecuación,

$$
M \omega=\int_{S C}\left(\frac{1}{2} \rho v^{2}\right)(\bar{v} \overline{d s})
$$

Donde $\mathrm{M}$ es el momento o par que hace girar la pala y $\omega$ es la velocidad de rotación del rotor. Es decir, que el flujo de energía cinética a través de la superficie de control es igual a la energía de giro de la pala. $\mathrm{Si}$, como en los apartados anteriores, se desglosa la integral anterior, se obtiene la expresión,

$$
\begin{gathered}
\int_{S C}\left(\frac{1}{2} \rho v^{2}\right)(\bar{v} \overline{d s})=\int_{A B C D}\left(\frac{1}{2} \rho v^{2}\right)(\bar{v} \overline{d s})+\int_{E F G H}\left(\frac{1}{2} \rho v^{2}\right)(\bar{v} \overline{d s})+\int_{B C F G}\left(\frac{1}{2} \rho v^{2}\right)(\bar{v} \overline{d s})+\int_{A D E H}\left(\frac{1}{2} \rho v^{2}\right)(\bar{v} \overline{d s})+\int_{A B F E}\left(\frac{1}{2} \rho v^{2}\right)(\bar{v} \overline{d s}) \\
+\int_{C D G H}\left(\frac{1}{2} \rho v^{2}\right)(\bar{v} \overline{d s}) \\
\int_{A B C D}\left(\frac{1}{2} \rho v^{2}\right)(\bar{v} \overline{d s})=-\frac{1}{2} \rho \omega^{3} \int_{a}^{b} r^{3} d r \int_{-\frac{\pi a}{Z}}^{\frac{\pi a}{Z}} d z=-\frac{\pi a}{4 Z} \rho \omega^{3}\left(b^{4}-a^{4}\right) \\
\int_{E F G H}\left(\frac{1}{2} \rho v^{2}\right)(\bar{v} \overline{d s})=\frac{1}{2} \rho \int_{a}^{b} \int_{-\frac{\pi a}{Z}}^{\frac{\pi a}{Z}}\left(v_{\omega r}^{*}+v_{r}^{2}+v_{z}^{2}\right)\left(v_{\omega r}^{*} d r d z\right) \\
\int_{B C F G}\left(\frac{1}{2} \rho v^{2}\right)(\bar{v} \overline{d s})=-\frac{1}{2} \rho U^{3} \int_{a}^{b} d r \int_{0}^{\theta} r d \theta=-\frac{\pi a}{Z} \rho U^{3}(b-a) \\
\int_{A D E H}\left(\frac{1}{2} v^{2}\right)(\bar{v} \overline{d s})=\frac{1}{2} \rho \int_{a}^{b} U^{* 3} d r \int_{0}^{\theta} r d \theta=\frac{\pi a}{Z} \rho \int_{a}^{b} U^{* 3} d r
\end{gathered}
$$

De igual modo que en los apartado anteriores, se considera que el flujo de energía a través de las caras ABFE y CDGH es despreciable.

$$
\begin{aligned}
& \int_{A B F E}\left(\frac{1}{2} v^{2}\right)(\bar{v} \overline{d s}) \cong 0 \\
& \int_{C D G H}\left(\frac{1}{2} v^{2}\right)(\bar{v} \overline{d s}) \cong 0
\end{aligned}
$$

La ecuación Ec. 60 se puede desglosar, a su vez, en los siguientes sumandos,

$$
\begin{aligned}
\int_{E F G H}\left(\frac{1}{2} \rho v^{2}\right)(\bar{v} \overline{d s})= & \frac{1}{2} \rho \int_{a}^{b} \int_{-\frac{\pi a}{Z}}^{\frac{\pi a}{Z}}\left(v_{\omega r}^{*}{ }^{2}+v_{r}^{2}+v_{z}^{2}\right)\left(v_{\omega r}^{*} d r d z\right) \\
& =\frac{1}{2} \rho \int_{a}^{b} \int_{-\frac{\pi a}{Z}}^{\frac{\pi a}{Z}}\left(v_{\omega r}^{*}\right)(d r d z)+\frac{1}{2} \rho \int_{a}^{b} v_{\omega r}^{*} d r \int_{-\frac{\pi a}{Z}}^{\frac{\pi a}{Z}}\left(v_{r}^{2}\right)(d z)+\frac{1}{2} \rho \int_{a}^{b} v_{\omega r}^{*} d r \int_{-\frac{\pi a}{Z}}^{\frac{\pi a}{Z}}\left(v_{z}^{2}\right)(d z)
\end{aligned}
$$


Sustituyendo las expresiones de $v_{\mathrm{r}}$ y $\mathrm{v}_{\mathrm{z}}$ (ecuaciones Ec. 27 y Ec. 28),

$$
\begin{aligned}
& \frac{1}{2} \rho \int_{a}^{b} v_{\omega r}^{*} d r \int_{-\frac{\pi a}{Z}}^{\frac{\pi a}{Z}}\left(v_{r}{ }^{2}\right)(d z)=\frac{1}{2} \rho \int_{a}^{b} v_{\omega r}^{*} d r \int_{-\frac{\pi a}{Z}}^{\frac{\pi a}{Z}}\left(\frac{\Gamma}{2 \pi}\left[\frac{z}{(r-a)^{2}+z^{2}}-\frac{z}{(r-b)^{2}+z^{2}}\right]\right)^{2}(d z) \\
& \frac{1}{2} \rho \int_{a}^{b} v_{\omega r}^{*} d r \int_{-\frac{\pi a}{Z}}^{\frac{\pi a}{Z}}\left(v_{z}^{2}\right)(d z)=\frac{1}{2} \rho \int_{a}^{b} v_{\omega r}^{*} d r \int_{-\frac{\pi a}{Z}}^{\frac{\pi a}{Z}}\left(\frac{\Gamma}{2 \pi}\left[-\frac{r-a}{(r-a)^{2}+z^{2}}+\frac{r-b}{(r-b)^{2}+z^{2}}\right]\right)^{2}(d z)
\end{aligned}
$$

Si para simplificar las expresiones anteriores, se definen las funciones,

$$
\begin{gathered}
\mu(r)=\int_{-\frac{\pi a}{Z}}^{\frac{\pi a}{Z}}\left(\frac{z}{(r-a)^{2}+z^{2}}-\frac{z}{(r-b)^{2}+z^{2}}\right)^{2} d z \\
\lambda(r)=\int_{-\frac{\pi a}{Z}}^{\frac{\pi a}{Z}}\left(-\frac{r-a}{(r-a)^{2}+z^{2}}+\frac{r-b}{(r-b)^{2}+z^{2}}\right)^{2} d z
\end{gathered}
$$

Finalmente la ecuación del balance de energía queda,

$$
\begin{gathered}
M \omega=-\frac{\pi a}{4 Z} \rho \omega^{3}\left(b^{4}-a^{4}\right)+\rho \frac{\pi a}{Z} \int_{a}^{b} v_{\omega r}^{*} d r+\frac{1}{2} \rho\left(\frac{\Gamma}{2 \pi}\right)^{2} \int_{a}^{b} v_{\omega r}^{*}[\mu(r)+\lambda(r)] d r+\frac{\pi a}{Z} \rho \int_{a}^{b} U^{* 3} d r \\
-\frac{\pi a}{Z} \rho U^{3}(b-a)
\end{gathered}
$$

Despejando la circulación $\Gamma$,

$$
\Gamma=2 \pi \sqrt{\frac{\frac{2 M \omega}{\rho}+\frac{\pi a}{2 Z} \omega^{3}\left(b^{4}-a^{4}\right)-\frac{2 \pi a}{Z} \int_{a}^{b} v_{\omega r}^{*} d r-\frac{2 \pi a}{Z} \int_{a}^{b} U^{* 3} d r+\frac{2 \pi a}{Z} U^{3}(b-a)}{\int_{a}^{b} v_{\omega r}^{*}[\mu(r)+\lambda(r)] d r}}
$$




\subsubsection{APLICACIÓN DE LA TEORÍA DE ELEMENTOS DE PALA}

Antes de continuar con el desarrollo matemático iniciado en los apartados anteriores, hay que hacer un inciso la teoría bidimensional de elementos de pala, y cómo se relacionan las fuerzas de cada sección radial de pala, con las ecuaciones obtenidas en los apartados anteriores.

En esta teoría, se establece que las fuerzas que aparecen en un perfil bidimensional, que se encuentra en un fluido en movimiento de velocidad $V$, son dos, una que es perpendicular $L$ (en inglés "Lift") a la velocidad incidente en la sección y otra paralela a ésta $D$ (en inglés "Drag").

El sentido y dirección de la fuerza $D$ es igual al de la velocidad $V$, y el de la fuerza $L$, perpendicular a la velocidad $V$. El sentido del vector $L$ depende de la orientación del perfil, tal y como se ve en la Figura 18. Las magnitudes de $L$ y $D$ vienen dadas por la expresiones [9],

$$
\begin{aligned}
L & =\frac{1}{2} \rho c_{L} c V^{2} \\
D & =\frac{1}{2} \rho c_{D} c V^{2}
\end{aligned}
$$

$\rho$ es la densidad del fluido, c es la cuerda o longitud de la sección como se muestra en la Figura 18 y por último, $\mathrm{c}_{\mathrm{L}}$ y $\mathrm{c}_{\mathrm{D}}$ son coeficientes adimensionales que dependen de la geometría del perfil sustentador utilizado en la sección y del ángulo de ataque $\gamma$.

Estos coeficientes están tabulados en numerosas publicaciones, y el aspecto genérico de las distribuciones con respecto al ángulo de ataque $\gamma$ se puede observar en la Figura 17. En ella se ve que el valor de $\mathrm{c}_{\mathrm{L}}$ va creciendo hasta los 10 ó 15 grados, a partir de ahí se produce una caída brusca, en este momento se considera que el perfil ha entrado en pérdida (en inglés "stall"), haciendo por tanto que el valor de la fuerza L también decrezca abruptamente. Por lo que para que el perfil sustentador funcione correctamente, el ángulo de ataque no debe sobrepasar este valor crítico.

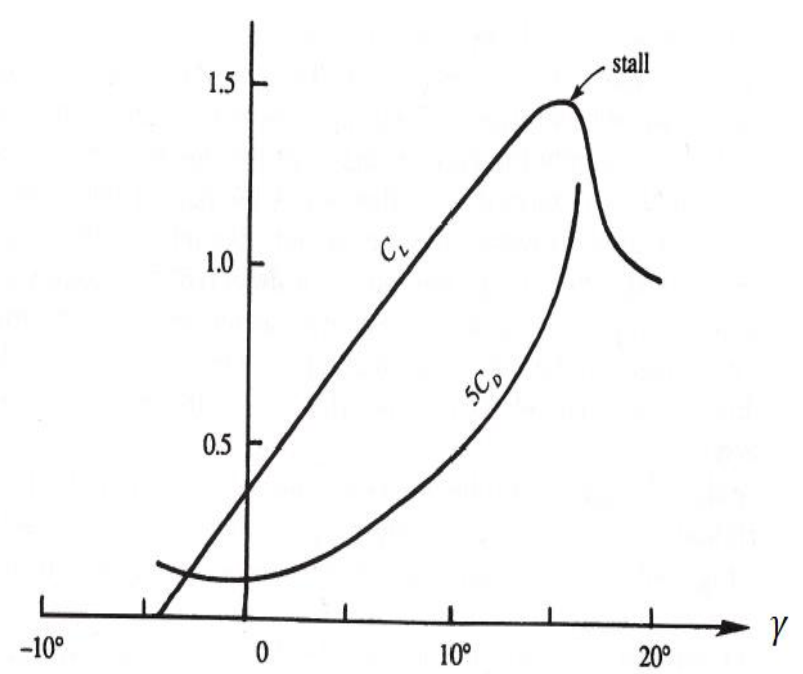

Figura 17. Distribución típica de los coeficientes de sustentación y arrastre [9] 

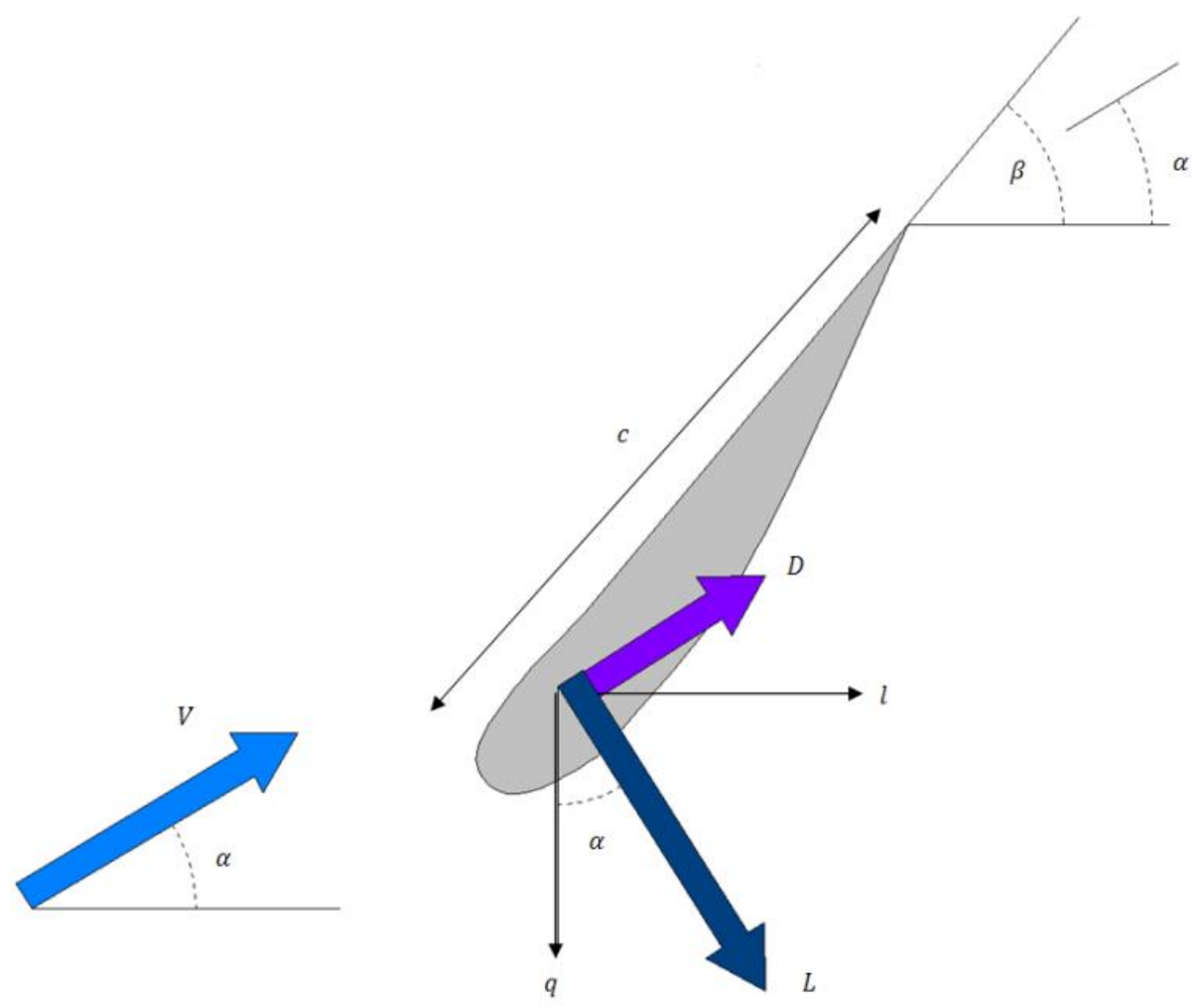

Figura 18. Disposición de las fuerzas de sustentación y de arrastre

Por otro lado, si $\beta$ es el ángulo que forma la sección con la horizontal que es conocido y viene definido por la geometría de la pala, y $\alpha$ el que forma la velocidad $V$ con la horizontal, como se ve en la Figura 18, entonces el ángulo de ataque es,

$$
\gamma=\beta-\alpha
$$

Si se proyectan $\mathrm{L}$ y $\mathrm{D}$ en las direcciones perpendicular y paralela al sentido de giro y se suman estas componentes [20],

$$
\begin{aligned}
q & =\frac{1}{2} \rho c_{L} c V^{2} \cos \alpha-\frac{1}{2} \rho c_{D} c V^{2} \operatorname{sen} \alpha=\frac{1}{2} \rho c V^{2}\left(c_{L} \cos \alpha-c_{D} \operatorname{sen} \alpha\right) \\
l & =\frac{1}{2} \rho c_{L} c V^{2} \operatorname{sen} \alpha+\frac{1}{2} \rho c_{D} c V^{2} \cos \alpha=\frac{1}{2} \rho c V^{2}\left(c_{L} \operatorname{sen} \alpha+c_{D} \cos \alpha\right)
\end{aligned}
$$

q es la fuerza que produce el giro de la sección y por tanto la rotación del rotor. Y la suma de las fuerzas l de cada sección se tiene que compensar con la reacción de la pala en el núcleo del rotor.

\section{Corrección por pérdidas en la punta de pala [40]}

Para la correcta implementación de Método de Elementos de Pala (BEM) se introduce una corrección por pérdidas en la punta de pala, esta corrección se obtiene multiplicando las ecuaciones de q y l por el factor $\varepsilon$, que es igual a, 


$$
\varepsilon_{1}=\frac{2}{\pi} \operatorname{arcos}\left\{\exp \left[-\frac{\frac{Z}{2}\left(1-\frac{r}{b}\right)}{\left(\frac{r}{b}\right) \cos (\alpha)}\right]\right\}
$$

\section{Corrección por pérdidas en el extremo de núcleo de rotor [40]}

Del mismo modo que se introduce una corrección por pérdidas en el extremo de pala, se introduce igualmente otro término para evaluar las pérdidas en el núcleo del rotor

$$
\varepsilon_{2}=\frac{2}{\pi} \operatorname{arcos}\left\{\exp \left[\frac{\frac{Z}{2}\left(1-\frac{r}{a}\right)}{\left(\frac{r}{a}\right) \cos (\alpha)}\right]\right\}
$$

Por tanto la corrección total aplicada es,

$$
\varepsilon=\varepsilon_{1} \varepsilon_{2}
$$

Con lo que,

$$
\begin{aligned}
q & =\varepsilon \frac{1}{2} \rho c V^{2}\left(c_{L} \cos \alpha-c_{D} \operatorname{sen} \alpha\right) \\
l & =\varepsilon \frac{1}{2} \rho c V^{2}\left(c_{L} \operatorname{sen} \alpha+c_{D} \cos \alpha\right)
\end{aligned}
$$

Ec. 81

\section{Coeficientes de sustentación y arrastre ánqulos mayores al de pérdida [20]}

En el caso de que el ángulo de ataque sobre pase al ángulo de pérdida del perfil en estudio, se implementan las siguientes curvas para obtener los coeficientes de sustentación y arrastre, siendo $\gamma_{S}$ el ángulo de pérdida, se utilizan las siguientes ecuaciones,

En primer lugar hay que obtener el máximo coeficiente de arrastre que es igual a,

$$
c_{D_{M A X}}=1,11+0,018 \cdot \frac{b-a}{c_{k}}
$$

Con esto se calculan las variables auxiliares A y B, para cada sección de pala,

$$
\begin{gathered}
A_{k}=\frac{\operatorname{sen}\left(\gamma_{s}\right)}{\cos ^{2}\left(\gamma_{s}\right)}\left[c_{L}\left(\gamma_{s}\right)-c_{D_{\text {MAX }}} \operatorname{sen}\left(\gamma_{s}\right) \cos \left(\gamma_{s}\right)\right] \\
B_{k}=\frac{1}{\cos \left(\gamma_{s}\right)}\left[c_{D}\left(\gamma_{s}\right)-c_{D_{M A X}} \operatorname{sen}^{2}\left(\gamma_{s}\right)\right]
\end{gathered}
$$


Finalmente los coeficientes de sustentación y arrastre para valores del ángulo de ataque superiores al ángulo de pérdida, es decir que $\gamma>\gamma_{s}$ son los siguientes,

$$
\begin{aligned}
& c_{L_{k}}=\frac{c_{D_{M A X_{k}}}}{2} \operatorname{sen}^{2}\left(\gamma_{k}\right)+A_{k} \frac{\cos ^{2}\left(\gamma_{k}\right)}{\operatorname{sen}\left(\gamma_{k}\right)} \\
& c_{D_{k}}=c_{D_{M A X_{k}}} \operatorname{sen}^{2}\left(\gamma_{k}\right)+B_{k} \operatorname{sen}\left(\gamma_{k}\right)
\end{aligned}
$$

Relacionando con las ecuaciones de los apartados anteriores, $\mathrm{F}_{\mathrm{z}}$ deberá ser igual opuesta que la resultante de fuerzas l,

$$
F_{z}=-\int_{a}^{b} l d r=-\rho \int_{a}^{b}\left[\frac{\Gamma}{\pi} v_{\omega r}^{*} \varphi(r)+\frac{2 \pi a}{Z}\left(U^{* 2}-U^{2}\right)\right] d r
$$

Desarrollando,

$$
\begin{gathered}
\frac{1}{2} \rho \int_{a}^{b} \varepsilon c V^{2}\left(c_{L} \operatorname{sen} \alpha+c_{D} \cos \alpha\right) d r=\rho \int_{a}^{b}\left[\frac{\Gamma}{\pi} v_{\omega r}^{*} \varphi(r)+\frac{2 \pi a}{Z}\left(U^{* 2}-U^{2}\right)\right] d r \\
\int_{a}^{b}\left[\frac{1}{2} \varepsilon c V^{2}\left(c_{L} \operatorname{sen} \alpha+c_{D} \cos \alpha\right)-\frac{\Gamma}{\pi} v_{\omega r}^{*} \varphi(r)-\frac{2 \pi a}{Z}\left(U^{* 2}-U^{2}\right)\right] d r=0
\end{gathered}
$$

Y análogamente,

$$
\begin{gathered}
F_{\theta}=\int_{a}^{b} q d r=\rho \frac{2 \pi a}{Z} \int_{a}^{b}\left(v_{\omega r}^{*}-\omega^{2} r^{2}\right) d r \\
\frac{1}{2} \rho \int_{a}^{b} \varepsilon c V^{2}\left(c_{L} \cos \alpha-c_{D} \operatorname{sen} \alpha\right) d r=\rho \frac{2 \pi a}{Z} \int_{a}^{b}\left(v_{\omega r}^{*}-\omega^{2} r^{2}\right) d r \\
\int_{a}^{b}\left[\frac{2 \pi a}{Z}\left(v_{\omega r}^{*}-\omega^{2} r^{2}\right)-\frac{1}{2} \varepsilon c V^{2}\left(c_{L} \cos \alpha-c_{D} \operatorname{sen} \alpha\right)\right] d r=0
\end{gathered}
$$

Puesto que, como se ha dicho, q es la fuerza que produce el giro de la sección, si M se definía en el apartado anterior como el momento o par de rotación, entonces se deduce que,

$$
M \omega=\omega \int_{a}^{b} r q d r
$$

Hay que tener en cuenta, y esto es muy importante, que el módulo de la velocidad $\mathrm{V}$ tiene que corregirse con los factores $s_{1}$ y $s_{2}$ de inducción axial y radial respectivamente, de esta forma $\mathrm{V}$ queda, 


$$
V \cong \sqrt{\left(1+s_{1}\right)^{2} U^{2}+\left(1+s_{2}\right)^{2}(\omega r)^{2}}
$$

Los factores de inducción se determinan con las siguientes ecuaciones que hay que resolver con un proceso iterativo,

$$
\begin{aligned}
& \frac{s_{1}}{1-s_{1}}=\left(\frac{Z c}{2 \pi r}\right) \frac{\left[c_{L} \operatorname{sen}(\alpha)+c_{D} \cos (\alpha)\right]}{4 \varepsilon \cos ^{2}(\alpha)} \\
& \frac{s_{2}}{1-s_{1}}=\left(\frac{Z c U}{2 \pi \omega r^{2}}\right) \frac{\left[c_{L} \cos (\alpha)-c_{D} \operatorname{sen}(\alpha)\right]}{4 \varepsilon \cos ^{2}(\alpha)}
\end{aligned}
$$

Y finalmente, el ángulo $\alpha$ se puede expresar en función del ángulo de ataque $\gamma$, de acuerdo a la ecuación Ec. 74,

$$
\alpha=\beta-\gamma
$$

\subsubsection{COEFICIENTE DE POTENCIA}

Con todo el desarrollo de los apartados anteriores, ya se tiene la información necesaria para poder saber si un determinado rotor cumple con el límite teórico de Betz. En primer lugar, se obtendrá la expresión del coeficiente $c_{P}$ de forma general, que es el cociente de la energía que aprovecha el rotor y la energía del fluido que produce la rotación del rotor.

La energía por unidad de tiempo que necesita el rotor para mantenerse en movimiento, es igual al momento o par de oposición que se genera en el eje, que viene dado por la expresión,

$$
Z M \omega
$$

Ec. 98

Esta expresión es análoga a la que se utilizó en el apartado 4.1.3 en el que se estudió el balance de energía, la diferencia es que en ese apartado se estudió solamente una pala y ahora hay que tener en cuenta todas las palas del rotor, de ahí que se haya multiplicado por el número de palas $\mathrm{Z}$.

Puesto que $\mathrm{M}$, como se ha dicho, es el par de oposición en el eje del rotor, para que éste pueda ser arrastrado por las fuerzas que se generan en la pala del rotor, como se ha visto en el apartado anterior, entonces la ecuación Ec. 98, queda de la forma,

$$
Z M \omega=Z \omega \int_{a}^{b} r q d r
$$

Por otro lado, la energía de la corriente del fluido, es igual a la energía cinética que atraviesa el disco del rotor en unidad de tiempo, que es,

$$
\frac{1}{2} \rho \pi b^{2} U^{3}
$$

Con lo cual el coeficiente $c_{P}$ queda así, 


$$
c_{P}=\frac{\mathrm{Z} \omega \int_{a}^{b} r q d r}{\frac{1}{2} \rho \pi b^{2} U^{3}}
$$

Desarrollando q de acuerdo a la ecuación Ec. 80 [43],

$$
c_{P}=\frac{\mathrm{Z} \omega \int_{a}^{b} \frac{1}{2} \rho r \varepsilon c V^{2}\left(c_{L} \cos \alpha-c_{D} \operatorname{sen} \alpha\right) d r}{\frac{1}{2} \rho \pi b^{2} U^{3}}=\frac{\mathrm{Z} \omega \int_{a}^{b} r \varepsilon c V^{2}\left(c_{L} \cos \alpha-c_{D} \operatorname{sen} \alpha\right) d r}{\pi b^{2} U^{3}}
$$

En este momento, observando la ecuación anterior, si se supone que el coeficiente de arrastre es nulo, la expresión resultante constituirá una cota superior del coeficiente de potencia real. Esta cota superior se supondrá que es aproximadamente el valor del máximo teórico del coeficiente de potencia y por tanto su valor nunca deberá ser mayor del límite de Betz, de este modo,

$$
\begin{gathered}
c_{P}=\frac{\mathrm{Z} \omega \int_{a}^{b} r \varepsilon c V^{2}\left(c_{L} \cos \alpha-c_{D} \operatorname{sen} \alpha\right) d r}{\pi b^{2} U^{3}} \leq c_{P}{ }^{*}=\frac{\mathrm{Z} \omega \int_{a}^{b} r \varepsilon c V^{2}\left(c_{L} \cos \alpha\right) d r}{\pi b^{2} U^{3}} \\
c_{P}{ }^{*}=\frac{\mathrm{Z} \omega \int_{a}^{b} r \varepsilon c V^{2}\left(c_{L} \cos \alpha\right) d r}{\pi b^{2} U^{3}} \leq 0,592
\end{gathered}
$$

En la práctica, existirán rotores definidos por las distribuciones de cuerdas, ángulos de ataque geométricos, el radio del núcleo, de la pala, etc. Que no serán compatibles con el límite teórico y que deben ser desechadas para su estudio.

\section{Coeficiente de potencia en función de la velocidad TSR}

Partiendo de la ecuación inicial del coeficiente de potencia, multiplicando y dividiendo por el radio del extremo de pala, se obtiene la expresión del coeficiente de potencia en función de la velocidad TSR,

$$
c_{P}=\frac{\mathrm{Z} \omega \mathrm{M}}{\frac{1}{2} \rho \pi b^{2} U^{3}}=\frac{\mathrm{Z} \omega \mathrm{bM}}{\frac{1}{2} \rho \pi b^{3} U^{3}}=\frac{\mathrm{ZM}(T S R)}{\frac{1}{2} \rho \pi b^{3} U^{2}}
$$

Derivando esta última expresión con respecto a TSR, se obtiene la expresión,

$$
\frac{d c_{P}}{d T S R}=\frac{Z}{\frac{1}{2} \rho \pi b^{3} U^{2}}\left(M+T S R \frac{d M}{d T S R}\right)
$$




\section{Tangente en el origen. Par de arranque.}

De acuerdo a la expresión anterior, la recta tangente en el origen tiene una inclinación con respecto a la horizontal igual a,

$$
\left.\frac{d c_{P}}{d T S R}\right|_{T S R=0}=\frac{Z}{\frac{1}{2} \rho \pi b^{3} U^{2}} M_{0}
$$

En este caso $\mathrm{M}_{0}$ es el momento o par de arranque del rotor, que es igual a la expresión del par, pero anulando la velocidad angular, esto es,

$$
M_{0}=\frac{1}{2} \rho \int_{a}^{b} r c U^{2}\left(c_{L} \cos \alpha-c_{D} \operatorname{sen} \alpha\right) d r
$$

\subsubsection{RESOLUCIÓN DE LAS ECUACIONES}

Una vez que se han obtenido las ecuaciones matemáticas que rigen el modelo, se procede en este apartado a resolverlas. Se trata de ecuaciones, como se ha visto, que contienen funciones integrales y que su resolución puede resultar complicada. En los próximos apartados, se utilizarán fórmulas aproximadas que permitan obtener resultados de manera rápida.

Sin embargo, antes de ese paso hay que realizar algunas consideraciones para no perder de vista el enfoque. En primer lugar, se van a recopilar las ecuaciones obtenidas ya combinadas con la teoría de perfil sustentador come se ha visto anteriormente.

La primera de ellas es la ecuación Ec. 39,

$$
\int_{a}^{b}\left(v_{\omega r}^{*}-\omega r+U^{*}-U\right) d r=0
$$

En el desarrollo del balance de momento lineal se obtuvieron dos ecuaciones, que luego en el apartado 4.1.4 se completaron con las expresiones de la teoría de perfil sustentador bidimensional (ecuaciones Ec. 89 y Ec. 92).

$$
\begin{gathered}
\int_{a}^{b}\left[\frac{1}{2} \varepsilon c V^{2}\left(c_{L} \operatorname{sen} \alpha+c_{D} \cos \alpha\right)-\frac{\Gamma}{\pi} v_{\omega r}^{*} \varphi(r)-\frac{2 \pi a}{Z}\left(U^{* 2}-U^{2}\right)\right] d r=0 \\
\int_{a}^{b}\left[\frac{2 \pi a}{Z}\left(v_{\omega r}^{*}{ }^{2}-\omega^{2} r^{2}\right)-\frac{1}{2} \varepsilon c V^{2}\left(c_{L} \cos \alpha-c_{D} \operatorname{sen} \alpha\right)\right] d r=0
\end{gathered}
$$

De la ecuación Ec. 89 se obtiene la circulación $\Gamma$,

$$
\Gamma=2 \pi \sqrt{\frac{\omega \int_{a}^{b} r \varepsilon c V^{2}\left(c_{L} \cos \alpha-c_{D} \operatorname{sen} \alpha\right) d r+\frac{\pi a}{2 Z} \omega^{3}\left(b^{4}-a^{4}\right)-\frac{2 \pi a}{Z} \int_{a}^{b} v_{\omega r}^{*}{ }^{3} d r-\frac{2 \pi a}{Z} \int_{a}^{b} U^{* 3} d r+\frac{2 \pi a}{Z} U^{3}(b-a)}{\int_{a}^{b} v_{\omega r}^{*}[\mu(r)+\lambda(r)] d r}}
$$


Y finalmente la ecuación de la compatibilidad con el límite teórico de Betz, del apartado anterior,

$$
c_{P}^{*}=\frac{\mathrm{Z} \omega \int_{a}^{b} \varepsilon c V^{2}\left(c_{L} \cos \alpha\right) d r}{\pi b^{2} U^{3}} \leq 0,592
$$

Estas son las ecuaciones que rigen el modelo matemático y para empezar a resolverlas, si se observan solamente las tres primeras, se ve que se trata de funciones integrales definidas en el intervalo $[\mathrm{a}, \mathrm{b}]$ e igualadas a cero. En principio, se puede decir que existirán infinitas funciones cuya integral sea nula en dicho intervalo, es decir, habrá infinitas soluciones del problema.

También será por tanto, solución la integral de la función nula, por lo que si se impone que los integrandos estas ecuaciones sean nulos, también se estará cumpliendo con la ecuación integral, esto es,

$$
\begin{gathered}
v_{\omega r}^{*}-\omega r+U^{*}-U=0 \rightarrow \int_{a}^{b}\left(v_{\omega r}^{*}-\omega r+U^{*}-U\right) d r=0 \\
\frac{1}{2} \varepsilon c V^{2}\left(c_{L} \operatorname{sen} \alpha+c_{D} \cos \alpha\right)-\frac{\Gamma}{\pi} v_{\omega r}^{*} \varphi(r)-\frac{2 \pi a}{Z}\left(U^{* 2}-U^{2}\right)=0 \\
\rightarrow \int_{a}^{b}\left[\frac{1}{2} \varepsilon c V^{2}\left(c_{L} \operatorname{sen} \alpha+c_{D} \cos \alpha\right)-\frac{\Gamma}{\pi} v_{\omega r}^{*} \varphi(r)-\frac{2 \pi a}{Z}\left(U^{* 2}-U^{2}\right)\right] d r=0 \\
\frac{2 \pi a}{Z}\left(v_{\omega r}^{*}-\omega^{2} r^{2}\right)-\frac{1}{2} \varepsilon c V^{2}\left(c_{L} \cos \alpha-c_{D} \operatorname{sen} \alpha\right)=0 \\
\rightarrow \int_{a}^{b}\left[\frac{2 \pi a}{Z}\left(v_{\omega r}^{*}-\omega^{2} r^{2}\right)-\frac{1}{2} \varepsilon c V^{2}\left(c_{L} \cos \alpha-c_{D} \operatorname{sen} \alpha\right)\right] d r=0
\end{gathered}
$$

Por otro lado, no hay que perder de vista cuáles van a ser las incógnitas a determinar. Si se tiene un determinado rotor, que queda definido por los parámetros geométricos que se muestran a continuación:

$$
\begin{array}{cl}
a & \text { radio del núcleo del rotor } \\
b & \text { radio del extremo de pala } \\
Z & \text { número de palas } \\
c(r) & \text { distribución radial de cuerdas } \\
\beta(r) & \text { distribución radial de ángulos de ataque geométricos de la pala }
\end{array}
$$

PERFIL distribución radial de perfiles sustentadores

Si se tiene en cuenta la ecuación Ec. 97 en la que se dedujo que $\alpha=\beta-\gamma$, se tiene que las variables desconocidas que aparecen en las ecuaciones son:

\footnotetext{
$\gamma \quad$ ángulo con respecto a la horizontal de la velocidad del fluido en cada radio $\mathbf{r}$

$v_{\omega r}^{*} \quad$ velocidad de salida del fluido según el volumen de control planteado en el apartado 5.2

$U^{*} \quad$ velocidad de salida del fluido según el volumen de control planteado en el apartado 5.2

$\Gamma \quad$ intensidad de circulación a la salida de la pala
} 
La forma de proceder es la siguiente:

\section{En primer lugar, obtener las variables auxiliares}

$$
\begin{gathered}
\varphi=\arctan \left[\frac{\pi a}{Z(b-r)}\right]+\arctan \left[\frac{\pi a}{Z(r-a)}\right] \\
\mu(r)=\int_{-\frac{\pi a}{Z}}^{\frac{\pi a}{Z}}\left(\frac{z}{(r-a)^{2}+z^{2}}-\frac{z}{(r-b)^{2}+z^{2}}\right)^{2} d z \\
\lambda(r)=\int_{-\frac{\pi a}{Z}}^{\frac{\pi a}{Z}}\left(-\frac{r-a}{(r-a)^{2}+z^{2}}+\frac{r-b}{(r-b)^{2}+z^{2}}\right)^{2} d z
\end{gathered}
$$

II. Calcular $\mathrm{v}_{\omega r}^{*}$ con la ecuación Ec. 119 y suponiendo un valor inicial de $\gamma$

$$
v_{\omega r}^{*}=\sqrt{\frac{Z}{4 \pi a} \varepsilon c V^{2}\left[c_{L} \cos (\beta-\gamma)-c_{D} \operatorname{sen}(\beta-\gamma)\right]+\omega^{2} r^{2}}
$$

\section{Calcular $\mathrm{U}^{*}$ con la ecuación Ec. 109}

$$
U^{*}=U+\omega r-v_{\omega r}^{*}
$$

IV. Obtener la circulación $\Gamma$

$$
\Gamma=2 \pi \sqrt{\frac{\omega \int_{a}^{b} r \varepsilon c V^{2}\left(c_{L} \cos \alpha-c_{D} \operatorname{sen} \alpha\right) d r+\frac{\pi a}{2 Z} \omega^{3}\left(b^{4}-a^{4}\right)-\frac{2 \pi a}{Z} \int_{a}^{b} v_{\omega r}^{*} d r-\frac{2 \pi a}{Z} \int_{a}^{b} U^{* 3} d r+\frac{2 \pi a}{Z} U^{3}(b-a)}{\int_{a}^{b} v_{\omega r}^{*}[\mu(r)+\lambda(r)] d r}}
$$

V. Se comprueba que la distribución de ángulos de ataque cumple con la ecuación Ec. 121

$$
\int_{a}^{b}\left[\frac{1}{2} \varepsilon c V^{2}\left(c_{L} \operatorname{sen} \alpha+c_{D} \cos \alpha\right)-\frac{\Gamma}{\pi} v_{\omega r}^{*} \varphi(r)-\frac{2 \pi a}{Z}\left(U^{* 2}-U^{2}\right)\right] d r=0
$$

Una solución particular de la ecuación anterior es que la que hace todos que la función del integrando sea nula, esto es:

$$
\frac{1}{2} c V^{2}\left[c_{L} \operatorname{sen}(\beta-\gamma)+c_{D} \cos (\beta-\gamma)\right]-\frac{\Gamma}{\pi} v_{\omega r}^{*} \varphi(r)-\frac{2 \pi a}{Z}\left(U^{* 2}-U^{2}\right)=0
$$

Si se denota la variable auxiliar $\psi(r)$ como,

$$
\psi(r)=\frac{1}{2} c V^{2}\left[c_{L} \operatorname{sen}(\beta-\gamma)+c_{D} \cos (\beta-\gamma)\right]-\frac{\Gamma}{\pi} v_{\omega r}^{*} \varphi(r)-\frac{2 \pi a}{Z}\left(U^{* 2}-U^{2}\right)
$$


Por tanto, una solución válida para la distribución de ángulos de ataque es la que haga que $\psi(r)=0$. Para cualquier valor de $r$. Aunque siempre hay que tener en cuenta que se trata de un sistema de ecuaciones que puede tener varias soluciones.

Hay que tener en cuenta que existen varias maneras de encontrar soluciones a las ecuaciones planteadas, en este caso por simplicidad se ha optado por la solución que hace nulo el integrando de las ecuaciones Ec. 109 y Ec. 119 y para no dejar fuera del conjunto de soluciones algunas que pueden ser válidas, en el caso de la ecuación Ec. 118 se deja la expresión general.

\subsubsection{RESOLUCIÓN NUMÉRICA}

Una vez obtenidas las ecuaciones que rigen este modelo, y una vez planteadas para resolución como se ha visto en el apartado anterior, es el momento de obtener resultados. Lo ideal sería obtener una solución general, sin embargo, esto, en principio, no resulta viable, debido a que la expresión de la circulación contiene funciones integrales dentro de una raíz cuadrada.

Por ello, parece que elaborar una solución numérica es lo más adecuado en este caso. Si se divide la pala en $n$ secciones radiales de espesor $\delta r$, en las que a cada una se asigna un subíndice $k$ que variará de 1 a $n$, en orden creciente empezando por la sección 1 que corresponde a la que está contigua al núcleo del rotor.

Entonces las integrales que aparecen en la ecuación Ec. 114 se pueden aproximar de la siguiente forma,

$$
\begin{gathered}
\int_{a}^{b} r c V^{2}\left(c_{L} \cos \alpha-c_{D} \operatorname{sen} \alpha\right) d r \cong \sum_{k=1}^{n} r_{k} c_{k} V_{k}^{2}\left(c_{L_{k}} \cos \left(\beta_{k}-\gamma_{k}\right)-c_{D_{k}} \operatorname{sen}\left(\beta_{k}-\gamma_{k}\right)\right) \delta r_{k} \\
\int_{a}^{b} v_{\omega r}^{*} d r \cong \sum_{k=1}^{n} v_{\omega r}^{*}{ }_{k}{ }_{k} \delta r_{k} \\
\int_{a}^{b} U^{* 3} d r \cong \sum_{k=1}^{n} U^{* 3}{ }_{k} \delta r_{k} \\
\int_{a}^{b} v_{\omega r}^{*}[\mu(r)+\lambda(r)] d r \cong \sum_{k=1}^{n} v_{\omega r_{k}}^{*}\left[\mu\left(r_{k}\right)+\lambda\left(r_{k}\right)\right] \delta r_{k}
\end{gathered}
$$

Dividir la pala en intervalos implica que el intervalo radial $\mathrm{b}-\mathrm{a}$ queda dividido en $\mathrm{n}$ sub-intervalos de longitud $\delta r_{k}$. Se puede tomar como valor representativo del radio $r_{k}$ en cada uno de los subintervalos de varias formas, aunque en este caso se escoge justo el valor medio, es decir, que los extremos de los sub-intervalos de b - a son,

$$
\left[r_{k}-\frac{\delta r_{k}}{2} ; r_{k}+\frac{\delta r_{k}}{2}\right]
$$

Y por otro lado se tiene que cumplir, de forma general que, 


$$
\sum_{k=1}^{n} \delta r_{k}=b-a
$$

Aunque en la mayoría de los casos se utilizará una división en la que la longitud de cada uno de los sub-intervalos es en partes iguales, esto es,

$$
\delta r_{k}=\frac{b-a}{n}
$$

Hay que tener en cuenta que cuanto mayor sea $n$, mayor será la precisión de los resultados obtenidos aunque también mayores los recursos de cálculo requeridos. En cada caso a calcular habrá que analizar qué valor de $n$ es el más adecuado.

El esquema de cálculo para obtener las soluciones numéricas de la distribución de ángulos de ataque $\gamma\left(\mathrm{r}_{\mathrm{k}}\right)$ es el mismo al del apartado anterior, sólo que hay que sustituir las funciones integrales de acuerdo a las ecuaciones planteadas más arriba. 


\subsubsection{ESQUEMA DE PASOS PARA LA RESOLUCIÓN NUMÉRICA}

\begin{tabular}{|l|}
$\begin{array}{l}\varphi\left(r_{k}\right)=\arctan \left[\frac{\pi a}{Z\left(b-r_{k}\right)}\right]+\arctan \left[\frac{\pi a}{Z\left(r_{k}-a\right)}\right] \\
\text { variables auxiliares }\end{array}$ \\
$\mu\left(r_{k}\right)=\int_{-\frac{\pi a}{Z}}^{\frac{\pi a}{Z}}\left(\frac{z}{\left(r_{k}-a\right)^{2}+z^{2}}-\frac{z}{\left(r_{k}-b\right)^{2}+z^{2}}\right)^{2} d z$ \\
$\lambda\left(r_{k}\right)=\int_{-\frac{\pi a}{Z}}^{\frac{\pi a}{Z}}\left(-\frac{r_{k}-a}{\left(r_{k}-a\right)^{2}+z^{2}}+\frac{r_{k}-b}{\left(r_{k}-b\right)^{2}+z^{2}}\right)^{2} d z$
\end{tabular}

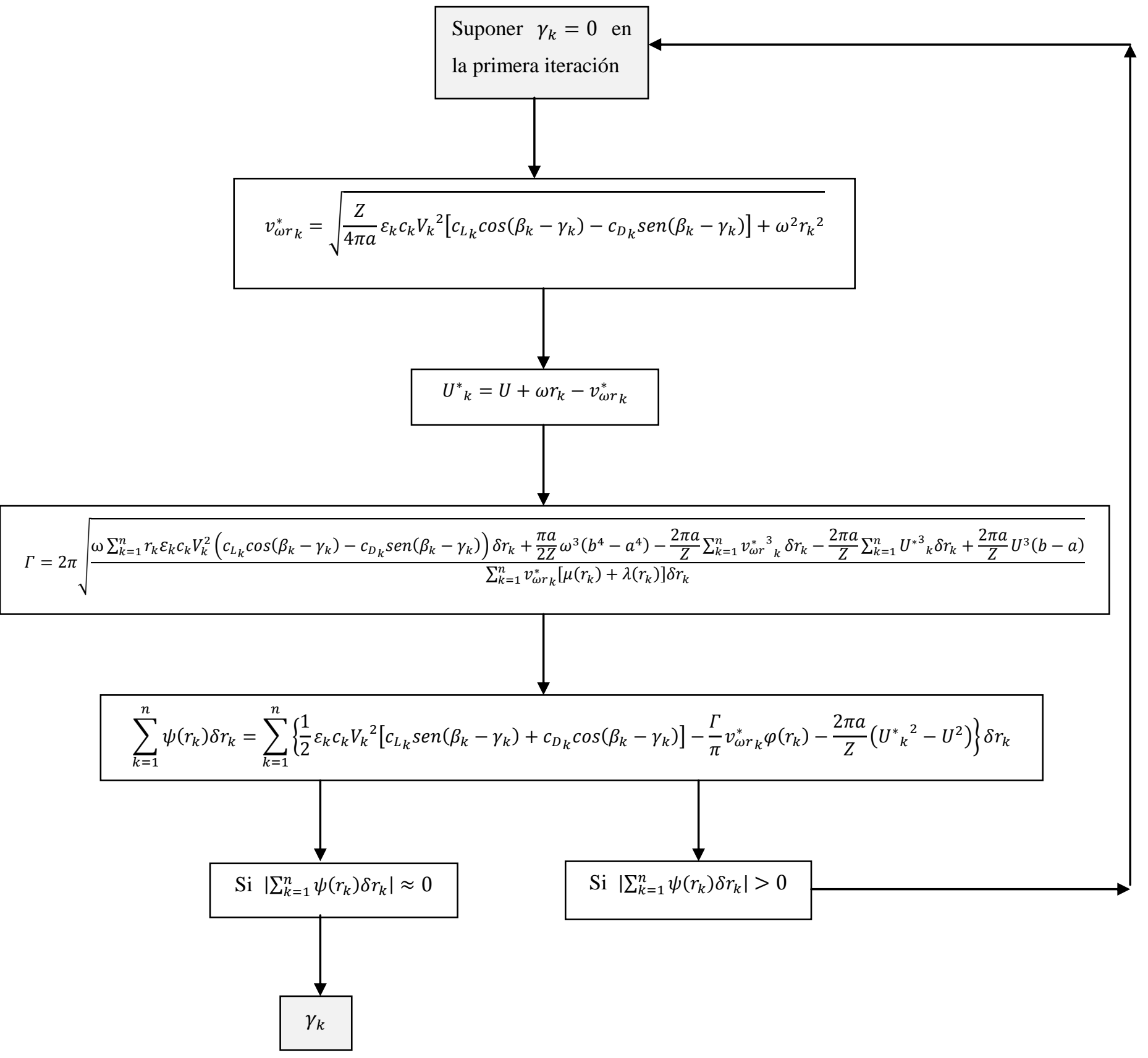




\subsubsection{OPTIMIZACIÓN}

La geometría de la pala del rotor se puede modificar de forma que se aproveche la mayor cantidad de energía de la corriente. El criterio a seguir para discernir si una geometría es mejor que otra, es mediante el cálculo del coeficiente $\mathrm{c}_{\mathrm{P}}^{*}$ de la ecuación Ec. 104, se supone que si este coeficiente es igual al límite teórico de Betz entonces la distribución de cuerdas y ángulos de ataque geométricos es óptima.

La forma de proceder para optimizar la distribución de cuerdas y ángulos de ataque de la pala consiste en a partir de unas distribuciones iniciales ir modificándolas de forma que el valor de $\mathrm{c}_{\mathrm{P}}^{*}$ sea lo más cercano a 0,592 que es el valor límite termodinámico. Al ir haciendo estas modificaciones, se influirá también en los ángulos de ataque $\gamma\left(\mathrm{r}_{\mathrm{k}}\right)$ que hacen que $\psi\left(\mathrm{r}_{\mathrm{k}}\right)$ sea lo más cercano a cero posible.

Por tanto, el proceso de optimización puede ser un poco laborioso aunque se facilita mucho una vez que el procedimiento de cálculo esté implementado en una hoja de cálculo. Se supone que para la optimización los valores del radio del núcleo del rotor, el radio total del rotor, la velocidad de la corriente y la velocidad de giro son dadas, es decir, se mantendrán constantes durante los cálculos.

De este modo la optimización puede representarse como una espiral, ya que se puede repetir tantas veces como se quiera, en la que uno de los vértices lo constituyen las distribuciones de cuerdas y ángulos de ataque geométricos, el siguiente vértice serían los ángulos de ataque de cada sección y por último el coeficiente $\mathrm{c}_{\mathrm{P}}^{*}$.

$$
\left\{c_{p}^{*}\right\} \quad\left\{c_{k} ; \beta_{k}\right\}
$$

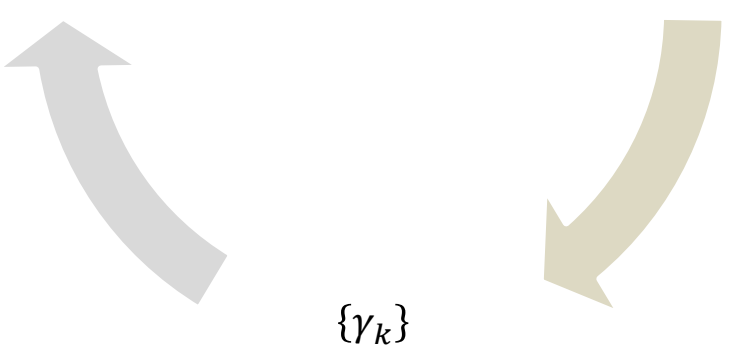

Figura 19. Espiral para la optimización de la geometría de la pala 
Así que una vez que se tienen unas distribuciones de cuerdas y ángulos de ataque geométricos en la pala, ya se consideran fijas y es entonces cuando se calcularán las nuevas distribuciones de ángulos de ataque $\gamma\left(r_{k}\right)$ para diferentes velocidades de giro o velocidades de corriente y obtener el coeficiente de potencia del rotor en cada una de estas condiciones.

En numerosas publicaciones relacionadas con la materia utilizan la relación de velocidad de la pala en el extremo con la velocidad de la corriente o coeficiente TSR ("Tip Speed Ratio") [14], para valorar el comportamiento del rotor en distintas condiciones de funcionamiento. Suponiendo que la velocidad del rotación está en revoluciones por minuto, la expresión de la velocidad TSR (Ec. 19), tiene la siguiente expresión,

$$
\operatorname{TSR}=\frac{2 \pi}{60} \frac{\omega b}{U}
$$

La velocidad nominal de giro y la velocidad de la corriente son parámetros importantísimos en el diseño y optimización del rotor. Hay que tener en cuenta que la eficiencia del rotor va a decrecer cuando el coeficiente TSR sea mayor o menor del valor nominal. Es decir, cuando se optimiza se está posicionando el máximo de la eficiencia del rotor.

En la Figura 20 se muestra como varía el valor del coeficiente $c_{P}$ para distintos TSR. En ella se puede ver que existe un máximo en torno al valor TSR $=4,5$ y a partir de ahí $\mathrm{C}_{\mathrm{P}}$ decrece rápidamente ya sea para TSR mayores o menores.

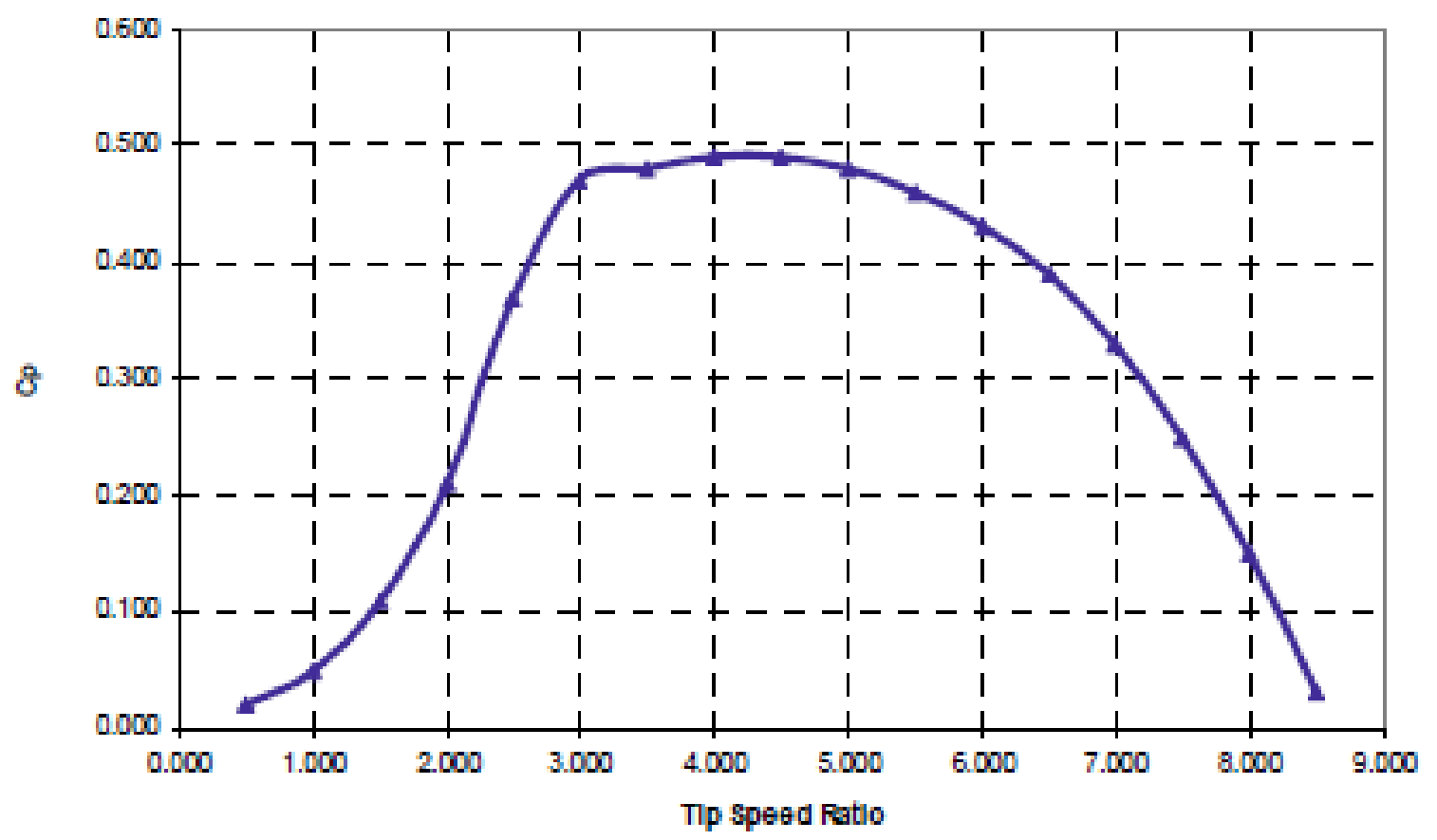

Figura 20. Gráfica tipo de representación del coeficiente de potencia con respecto a la TSR [16] 


\section{CAVITACIÓN}

El fenómeno de la cavitación fue observado por primera vez en una hélice de un barco a principios del siglo XX, y después de tiempo de estudio y experimentos, se llegó a la conclusión de que se produce cuando en alguna zona de un líquido, la presión baja por debajo de la presión de vapor a la temperatura correspondiente, originando burbujas de vapor que según sus características se pueden clasificar en varios tipos de cavitación (de extremo de pala, de hoja, de nube, etc. Figura 22).

Esto hace que, aunque la hélice de un barco y una turbina de corrientes marinas son dispositivos con diferentes parámetros de diseño y funcionamiento, se considera que estas últimas también pueden estar sometidas sus efectos, que van desde ruidos y vibraciones imprevistas en el diseño a erosión y daños superficiales en ciertas partes, que pueden llegar a ser graves.

Desde el punto de vista del impacto ambiental, el ruido ocasionado por las turbinas de corrientes marinas en las zonas donde estén instaladas, sobre todo si se trata de varias, es uno de los factores importantes a tener en cuenta ya que puede afectar a las especies marinas.

Por tanto, la cavitación [44, 45] es un fenómeno a evitar en cualquier turbina de corrientes marinas. Para ello como se ha dicho, hay que tener en cuenta la presión de las partículas fluidas en su tránsito a través del rotor, siendo las zonas en donde mayores velocidades se alcancen las que más probabilidad tienen de que aparezca la cavitación, ya que a mayor velocidad, menor presión.

En las publicaciones que tratan este fenómeno, como se mencionó anteriormente, utilizan un número adimensional conocido como número de cavitación dentado por la letra $\sigma$ y cuya expresión está recogida en la ecuación Ec. 20.
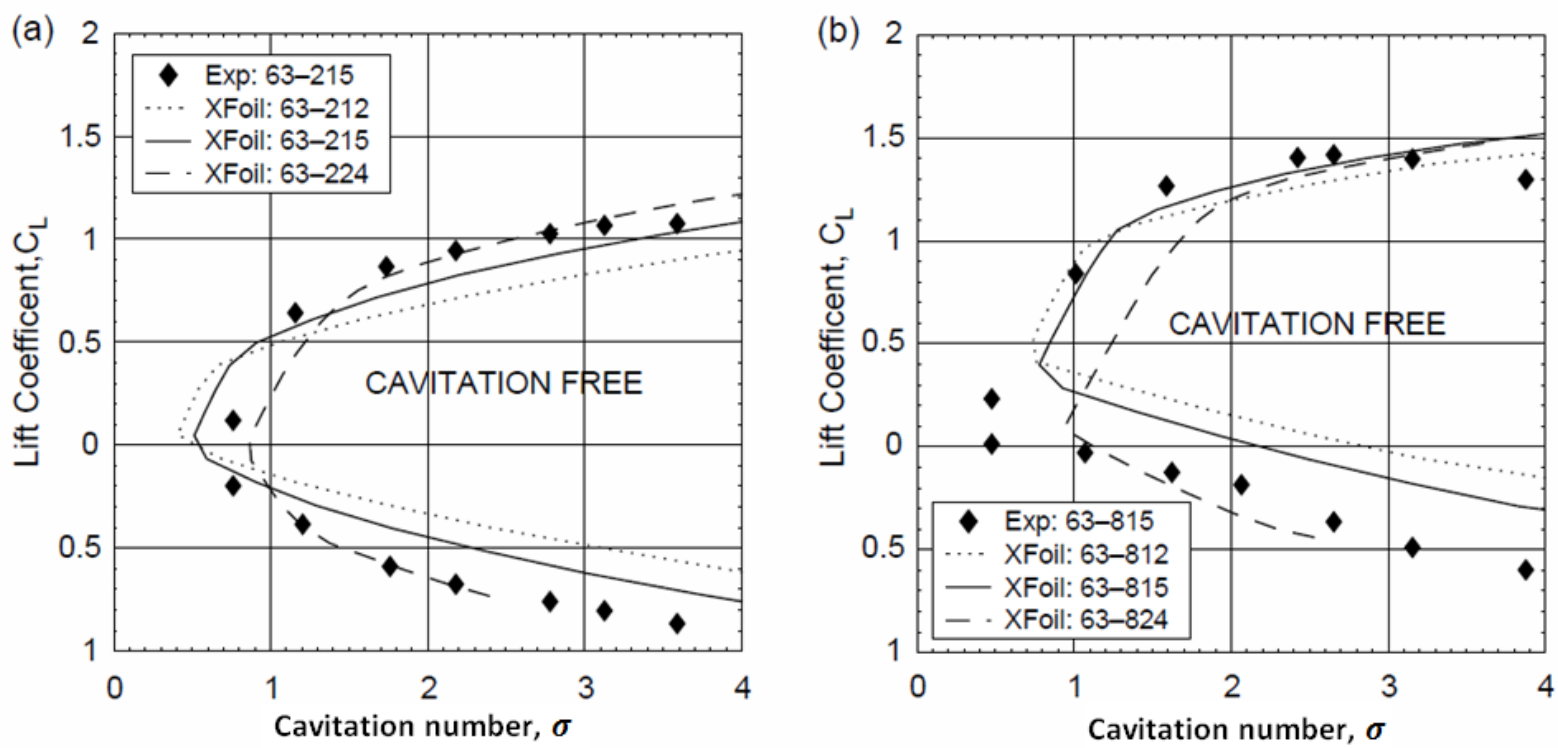

Figura 21. Gráficas tabuladas de cavitación para distintos perfiles sustentadores [46] 
Puesto que se trata de un problema de enorme complejidad, en esta tesis, solamente se utilizarán los las curvas de cavitación que complementan la Teoría de Elementos de pala en este aspecto.

Al igual que se realizan experimentos para tabular los valores de los coeficientes de sustentación y arrastre de un perfil sustentador bidimensional, también hay gráficos publicados basados en experimentos similares, que permiten conocer si en un determinado perfil se va producir cavitación según sean sus condiciones de funcionamiento.

Por ejemplo, si se observa la Figura 21, se ve que se han trazado dos gráficas, en las que en el eje horizontal representa el número de cavitación $\sigma$ y en el eje vertical en coeficiente de sustentación $\mathrm{c}_{\mathrm{L}}$ , en este plano se ha trazado una línea en base a datos experimentales que lo divide en dos regiones, una que se considera que no aparece la cavitación y otra que sí.

Así pues, estas gráficas tabuladas constituyen una potente herramienta para conocer si en alguna zona de la pala del rotor de originará cavitación.

Un aspecto importante a tener en cuenta con respecto a las gráficas descritas en el párrafo anterior, es que éstas están trazadas para perfiles sustentadores que se consideran bidimensionales, es decir, los experimentos se han realizado sobre un cuerpo de determinada longitud mucho mayor que las dimensiones de la sección transversal constante.

De este modo, no se tienen en cuenta los posibles efectos de la interacción entre zonas con distinta sección transversal como es el caso de las palas del rotor. Parta tratar de paliar el error que se pueda producir, existen genéricas de probabilidad que estiman si es probable o no que se produzca la cavitación, dependiendo de la profundidad de las partículas fluidas y su velocidad, como se ve en la Figura 9.

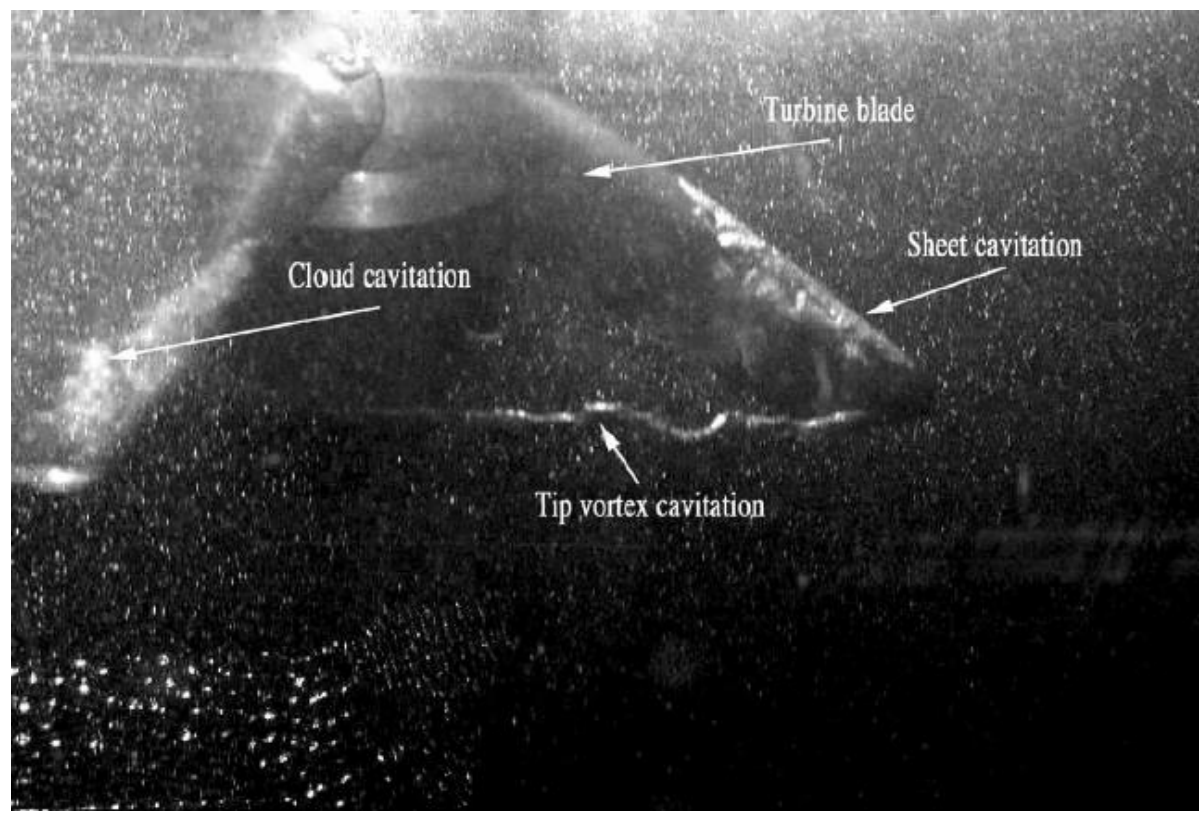

Figura 22. Distintos tipos de cavitación [21] 
La aportación que se hace en esta tesis es equivalente a la que se hace para calcular las cargas estáticas en la pala, es decir, tratar de modelar, con la hipótesis de estela de pala que se ha establecido en el apartado 4, las interacciones entre los distintos elementos de pala, de esta forma se corregirán los ángulos de ataque que se obtendrían con metodologías de cálculo basadas en la Teoría de Elementos de Pala, y de este modo la estimación de si se originará el fenómeno de cavitación en la pala del rotor será más precisa. 


\section{MODELADO ELÁSTICO DE LA PALA $[47,48,49,50,51,52]$}

\section{FLEXIÓN}

Con el procedimiento de cálculo propuesto en este documento, se van a obtener las cargas estáticas que se originan en la pala y con ellas se pueden estimar los elementos estructurales adecuados para su construcción, mediante la aplicación de la ecuación fundamental de la flexión [53],

$$
\frac{M_{\text {flector }}}{\sigma_{f}}=\frac{I_{\text {sección }}}{y}
$$

Donde para obtener el momento flector $\mathrm{M}_{\text {flector }}$, hay que calcular el esfuerzo cortante $\mathrm{E}_{\text {cortante, }}$ que en este caso es,

$$
\mathrm{E}_{\text {cortante }}\left(r_{k}\right)=\sum_{i=k}^{n} l_{i}
$$

$$
r_{k} l_{k}
$$

Y el momento flector sería,

$$
M_{\text {flector }}\left(r_{k}\right)=\sum_{i=k}^{n} r_{i} l_{i}
$$

El momento de inercia con respecto a un eje perpendicular a la dirección de deformación que pase

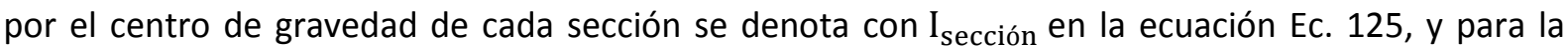
distancia a este eje se utiliza la variable y. Por último queda por definir $\sigma_{\mathrm{f}}$ que es un valor de la carga máxima admisible por el material y que normalmente corresponde al límite elástico del material utilizado multiplicado por un coeficiente de seguridad menor que la unidad.

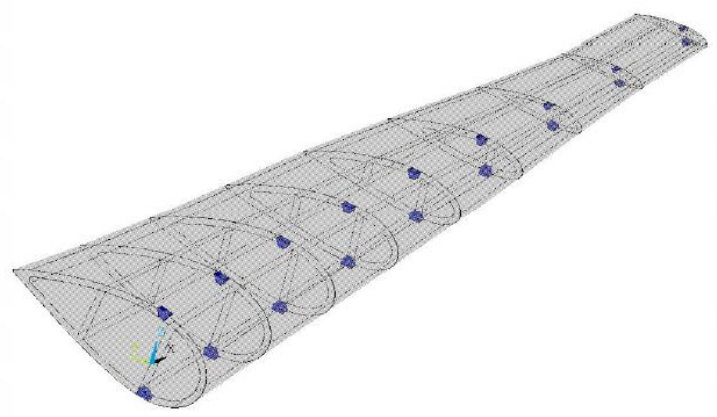

Figura 23. Disposición esquemática de refuerzos en la pala [54]

La configuración de refuerzos que se suele utilizar en la construcción de palas de rotor, es la de un refuerzo principal que recorre toda la longitud, colocado en el intervalo de $1 / 4$ y $1 / 3$ de la cuerda, 
midiendo estas distancias desde el borde de ataque, y que absorberá la mayor parte de los esfuerzos originados. También se añadirán otros refuerzos secundarios fijos al contorno interior de casa sección de pala y secciones transversales a lo largo de la pala.

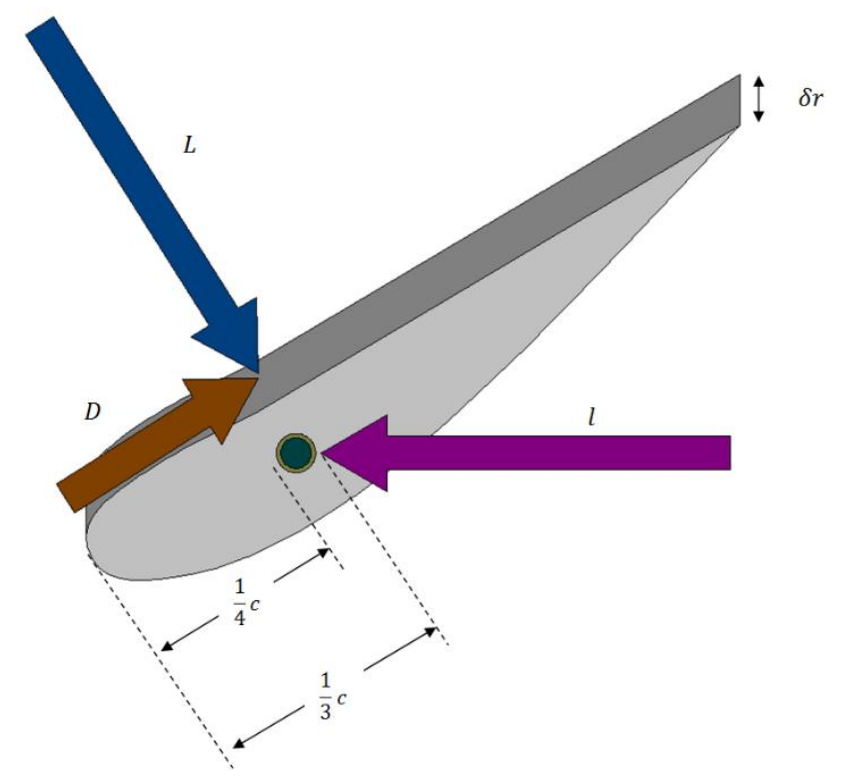

Figura 24. Punto de aplicación de las cargas en cada sección

\section{TORSIÓN [53, 55, 56]}

Cuando el fluido pasa a través de las palas del rotor, éste tiende a colocar la pala de forma que el empuje o la fuerza resultante en ellas sea nulo. Esta tendencia se expresa matemáticamente con el par de torsión que se aplica a cada sección bidimensional de la ecuación Ec. 16. Esto implica que la torsión de la pala afectará al coeficiente de potencia ya que se modifica la geometría de la pala. El par de torsión depende del coeficiente $c_{m}$, este coeficiente es función del ángulo de ataque y de la geometría del perfil sustentador utilizado.

De este modo, las cargas de torsión producirán un aumento en la distribución de ángulos de paso geométricos $\beta_{\mathrm{k}}$ [57], que por tanto a su vez, provocará que haya que recalcular los ángulos de ataque $\gamma_{\mathrm{k}}$, es decir, el fenómeno de torsión de la pala hace que la cargas estáticas tengan que obtenerse de forma iterativa de acuerdo a la nueva distribución de $\beta_{\mathrm{k}}^{\prime}$.

Para estimar el ángulo de torsión en la estructura de la pala se utilizarán las fórmulas de la Tabla 3. Estas fórmulas modelizan el comportamiento de distintos tipos de viga dependiendo de la geometría de la sección transversal. El ángulo de torsión de la estructura con respecto al de reposo viene dado por la ecuación [53],

$$
\theta=\frac{Q l}{K G}
$$


Donde $\mathrm{Q}$ es el momento de torsión total al que estaría sometida la viga genérica, donde l es la longitud de la viga, G [53] el módulo de rigidez del material, que depende del módulo de Young y del Módulo de Poisson [58], y K un factor de proporcionalidad que depende de la geometría de la estructura que se muestra en la Tabla 3.

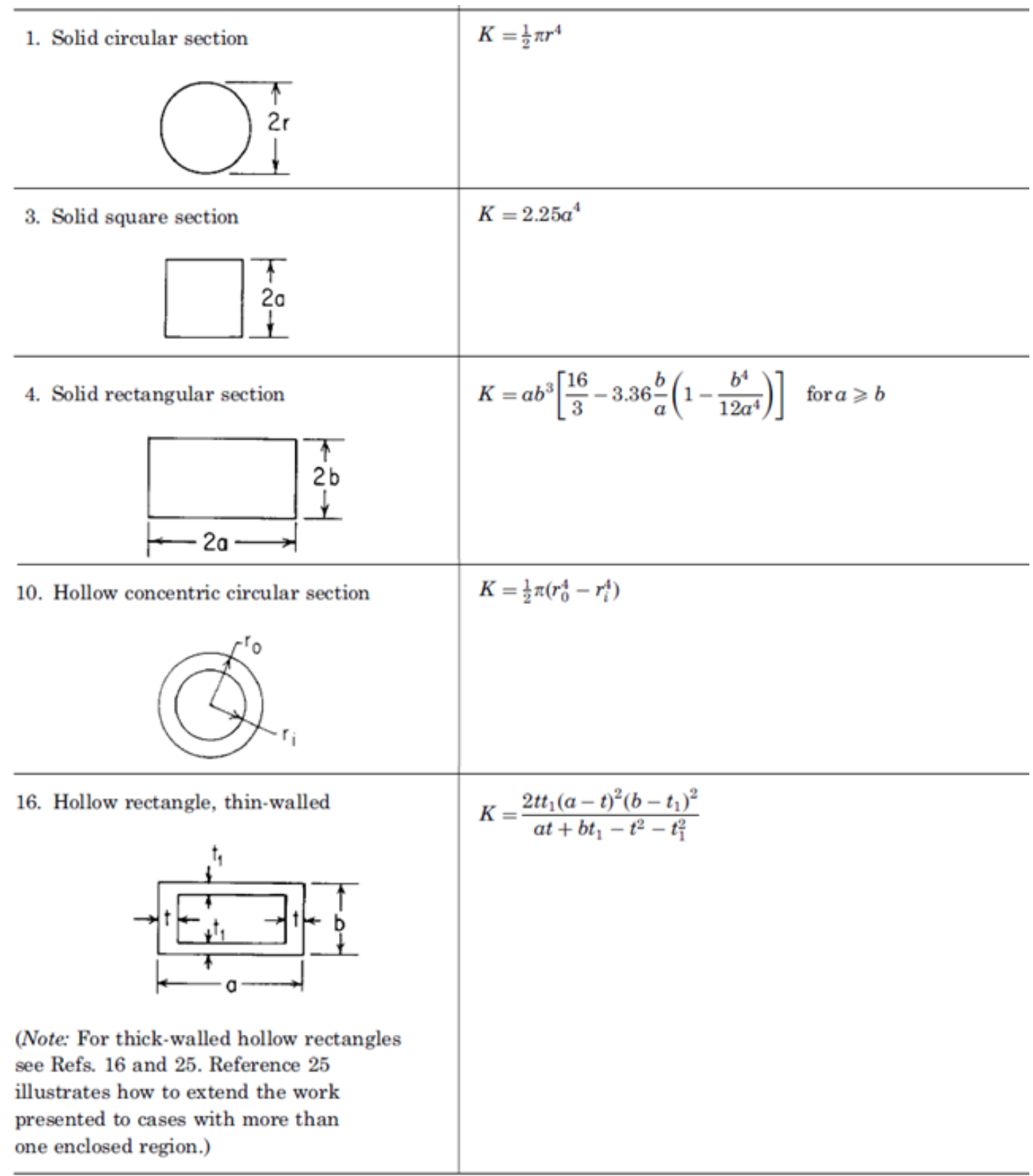

Tabla 3. Coeficiente de proporcionalidad K para distintas geometrías de sección de viga [52]

Para utilizar estas expresiones de la torsión hay que modelizar la estructura de la pala teniendo en cuenta que el refuerzo principal sea uno de los propuestos en la tabla anterior. De este modo, el par de torsión acumulado para cada sección de pala se puede escribir con la siguiente ecuación,

$$
Q_{k}=\frac{1}{2} \rho c_{m_{k}} c_{k}^{2} V_{k}^{2} \delta r_{k}+d_{k}\left(q_{k}+l_{k} \tan \beta_{k}\right)
$$


Siendo $\mathrm{d}_{\mathrm{k}}$ la distancia horizontal entre los puntos de aplicación de cargas hidrodinámicas y el centro de gravedad estructural de la sección. El momento de torsión de cada sección se compone de la suma del momento inherente a los perfiles sustentadores que se cuantifica con el sumando $\frac{1}{2} \rho c_{m_{k}} c_{k}{ }^{2} V_{k}^{2} \delta r_{k}$. Si el centro de aplicación de las fuerzas $l_{k} y d_{k}$ de la sección no coincide con el centro de gravedad estructural de la sección entonces estas fuerzas incrementan el momento de torsión.

El ángulo de torsión queda de la siguiente forma,

$$
\theta_{k}=\frac{\left[\sum_{i=1}^{k} Q_{i}\right] r_{k}}{K G}
$$

Y finalmente el nuevo ángulo de paso geométrico,

$$
\beta_{k}^{\prime}=\beta_{k}+\theta_{k}
$$




\section{IMPLEMENTACIÓN DE CASOS}

En este apartado se va implementar el procedimiento de cálculo desarrollado en los apartados anteriores aplicado a casos concretos de turbinas de corrientes marinas. De esta forma se podrá saber si los resultados que se obtengan reflejan la realidad de los fenómenos físicos que se producen durante el funcionamiento de estos dispositivos.

Para llevar a cabo esta tarea, la mejor opción sería la de contrastar los resultados obtenidos, con los parámetros de funcionamiento de una turbina que esté o haya estado en servicio. Sin embargo, el acceso a datos de esta índole es enormemente complicado, ya que las compañías y empresas que se dedican a esta actividad o parte de su actividad a las energías renovables marinas guardan gran recelo en exponer de forma pública los detalles de diseño, construcción y funcionamiento.

No obstante, y partiendo de esa idea inicial, se han encontrado algunas publicaciones académicas en las que se ofrece gran cantidad de información acerca diseños de turbinas en proceso de construcción o simplemente modelizadas por ordenador utilizando potentes herramientas informáticas. Por tanto, son estos datos los que se van a utilizar para comparar los resultados obtenidos mediante el procedimiento alternativo de cálculo propuesto en esta tesis.

Los resultados obtenidos con el procedimiento alternativo propuesto en esta tesis se compararán con los obtenidos con el método de elementos de pala [20], de esta forma se podrá conocer la precisión de éstos.

Las distribuciones de ángulos de ataque $\gamma_{k}$, se suponen por simplicidad de cálculo que van a ser lineales, donde el ángulo de ataque de extremo de pala $\gamma_{n}$ será aproximadamente el valor que anula el coeficiente de sustentación según el perfil utilizado en esa sección, es decir se busca $c_{L_{n}}\left(\gamma_{n}\right) \approx 0$.

En el último caso de aplicación, se implementará en procedimiento de cálculo para el generador GESMEY, se realizará adicionalmente, un estudio estructural y un diseño esquemático de los refuerzos que tiene que soportar las cargas que se aplican en la pala. Para ello se tendrá en cuenta la posibilidad de construir cada pala con distintos materiales.

A partir de los resultados obtenidos mediante el procedimiento alternativo de cálculo y el método de elementos de pala y su posterior análisis, se podrán sacar conclusiones acerca de la bondad de éstos y en su caso, plantear posibles mejoras a introducir en futuros trabajos.

El diseño de una turbina de corrientes conlleva escoger los parámetros de diseño del rotor que son el propio diámetro del rotor, el diámetro del núcleo de rotor ("hub" en inglés), el número de pala, las distribuciones radiales de ángulos de paso geométrico y de cuerdas, y finalmente la elección del perfil bidimensional para las secciones pala.

Todos estos parámetros se recogen en un fichero de datos separados por punto y coma (.csv) y se llevan directamente a las rutinas de cálculo. Los ficheros de datos relativos a los coeficientes de sustentación y arrastre se guardan también en un fichero. 
La elección del número de palas de una turbina se puede hacer en base a la siguiente TABLAXXX donde se da este número en función de la velocidad TSR de funcionamiento nominal de la turbina. Aunque para todos los casos que se van a estudiar a continuación el número de palas $Z$ es igual a 3 .

\begin{tabular}{|c|c|}
\hline TSR & $\boldsymbol{Z}$ \\
\hline 1 & $8-24$ \\
2 & $6-12$ \\
3 & $3-6$ \\
4 & $3-4$ \\
more than 4 & $1-3$ \\
\hline
\end{tabular}

Tabla 4. Número de palas recomendable en función de la velocidad TSR de diseño [14]

\subsection{EJEMPLO DE IMPLEMENTACIÓN PRELIMINAR 1 [14]}

Tomando como base el ejemplo de aplicación de la Teoría de Elementos de pala recogido en este documento [14], se confecciona una aplicación informática en lenguaje JAVA que implementa el método de cálculo basado en esta teoría y también el esquema de cálculos del apartado 4.1.7.1.

Este ejemplo servirá para depurar posibles errores en las ecuaciones y revisar posibles mejoras en el programa informático. Este programa se utilizará para implementar distintas geometrías de pala en los siguientes apartados, por lo que es esencial que depurar cualquier tipo de error que pueda desvirtuar los resultados, tanto los obtenidos a partir del método de elementos de pala como del procedimiento de esta tesis.

El ejemplo en cuestión está planteado para el caso de una turbina eólica, esto implica que hay que cambiar la densidad del fluido a $1,185 \mathrm{~kg} / \mathrm{m}^{3}$ que corresponde a la densidad del aire. La geometría de pala en cuestión está definida por el radio del núcleo de rotor $a=0,2 m$ y el radio del extremo de pala es $b=5 \mathrm{~m}$, el número de palas es igual a 3 .

Las condiciones de funcionamiento de la turbina consiste en una velocidad $U=7 \mathrm{~m} / \mathrm{s}$ y $107 \mathrm{rpm}$, que corresponde a una velocidad TSR de 8.

Las distribuciones radiales de ángulos de paso geométrico y cuerdas se recogen en la siguiente Tabla 5 .

Las curvas polares de coeficientes de sustentación y arrastre se han obtenido de la web [11], para el NACA0012.

Todos estos datos son recogidos por el programa de cálculo y se obtienen los resultados siguientes: El coeficiente de potencia es igual a 0,492 y la potencia de la turbina es de 7,8 kW. Estos valores son bastante aproximados a los esperados [14]. 
La distribución radial de ángulos de ataque, de coeficientes de sustentación y coeficientes de arrastre se muestran en la siguiente Tabla 6, Figura 25 y Figura 26.

\begin{tabular}{|c|c|c|c|c|c|}
\hline no & $\mathbf{r}[\mathbf{k}][\mathbf{m}]$ & delta[k][m] & bheta[k][0] & $\mathbf{c [ k ] [ m ]}$ & PERFIL \\
\hline 1 & 0,6 & 0,8 & 61 & 0,7 & NACA0012 \\
\hline 2 & 1,4 & 0,8 & 74,29 & 0,71 & NACA0012 \\
\hline 3 & 2,2 & 0,8 & 84,9 & 0,44 & NACA0012 \\
\hline 4 & 3 & 0,8 & 89,09 & 0,3 & NACA0012 \\
\hline 5 & 3,799 & 0,8 & 91,29 & 0,23 & NACA0012 \\
\hline 6 & 4,599 & 0,8 & 92,59 & 0,19 & NACA0012 \\
\hline
\end{tabular}

Tabla 5. Geometría de pala de la turbina en estudio

\begin{tabular}{|c|c|c|c|c|c|c|c|c|}
\hline $\mathrm{n}$ - & $\mathrm{r}[\mathrm{k}][\mathrm{m}]$ & delta[k][m] & bheta[k][o] & $\mathrm{c}[\mathrm{k}][\mathrm{m}]$ & PERFIL & $\mathrm{cl}[\mathrm{k}]$ & $\mathrm{cd}[\mathrm{k}]$ & gamma[k][o] \\
\hline 1 & 0,6 & 0,8 & 61 & 0,7 & NACA0012 & 0,521 & 0,097 & 12,93 \\
\hline 2 & 1,4 & 0,8 & 74,29 & 0,71 & NACA0012 & 0,421 & 0,014 & 2,59 \\
\hline 3 & 2,2 & 0,8 & 84,9 & 0,44 & NACA0012 & 0,759 & 0,023 & 6,95 \\
\hline 4 & 3 & 0,8 & 89,09 & 0,3 & NACA0012 & 0,869 & 0,03 & 8,27 \\
\hline 5 & 3,799 & 0,8 & 91,29 & 0,23 & NACA0012 & 0,898 & 0,032 & 8,64 \\
\hline 6 & 4,599 & 0,8 & 92,59 & 0,19 & NACA0012 & 0,902 & 0,033 & 8,7 \\
\hline
\end{tabular}

Tabla 6. Distribuciones de ángulos de ataque $\gamma_{k} \mathrm{rpm}=107, \mathrm{U}=7 \mathrm{~m} / \mathrm{s}$

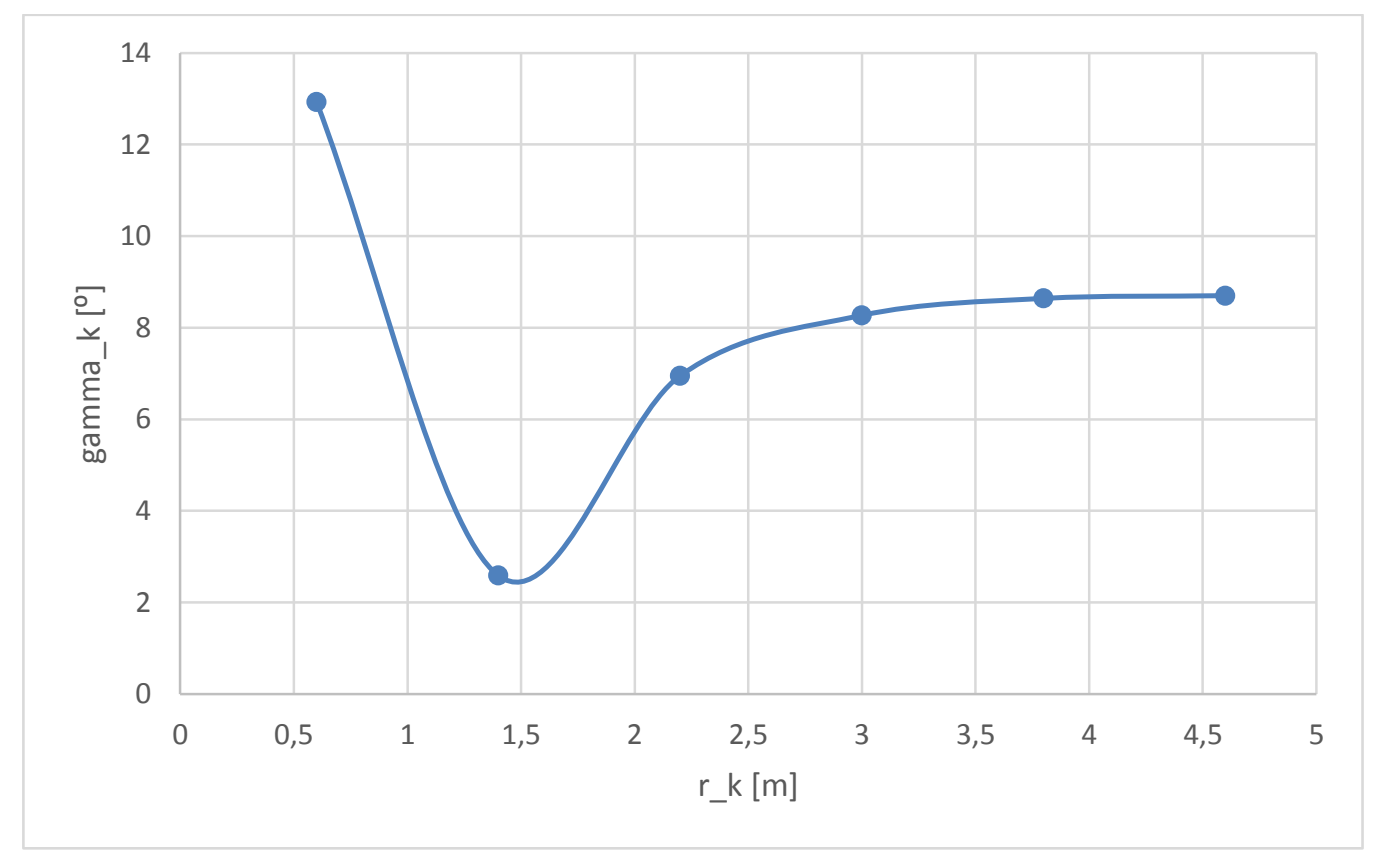

Figura 25. Representación gráfica de la distribución de ángulos de ataque $\gamma_{k} \mathrm{rpm}=107, \mathrm{U}=7 \mathrm{~m} / \mathrm{s}$ 


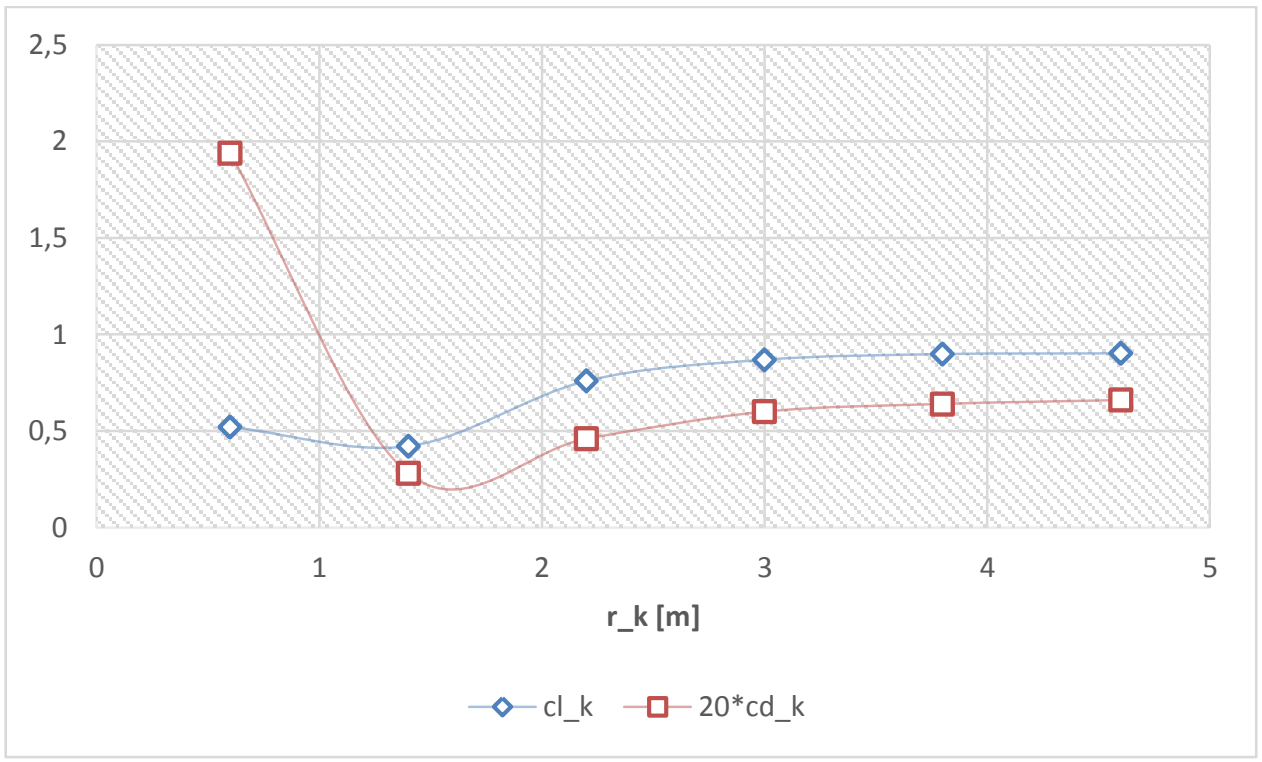

Figura 26. Representación gráfica de las distribuciones radiales de coeficientes de sustentación y arrastre $\mathrm{rpm}=107, \mathrm{U}=7 \mathrm{~m} / \mathrm{s}$

Las distribuciones de coeficientes de sustentación y arrastre calculados permiten obtener las cargas $\boldsymbol{l}_{\boldsymbol{k}}$ y $\boldsymbol{q}_{\boldsymbol{k}}$ a las que están sometidas las secciones de pala y que posteriormente servirán para realizar el análisis estructural de la pala. Las distribuciones de estas cargas se muestran en la Figura 27.

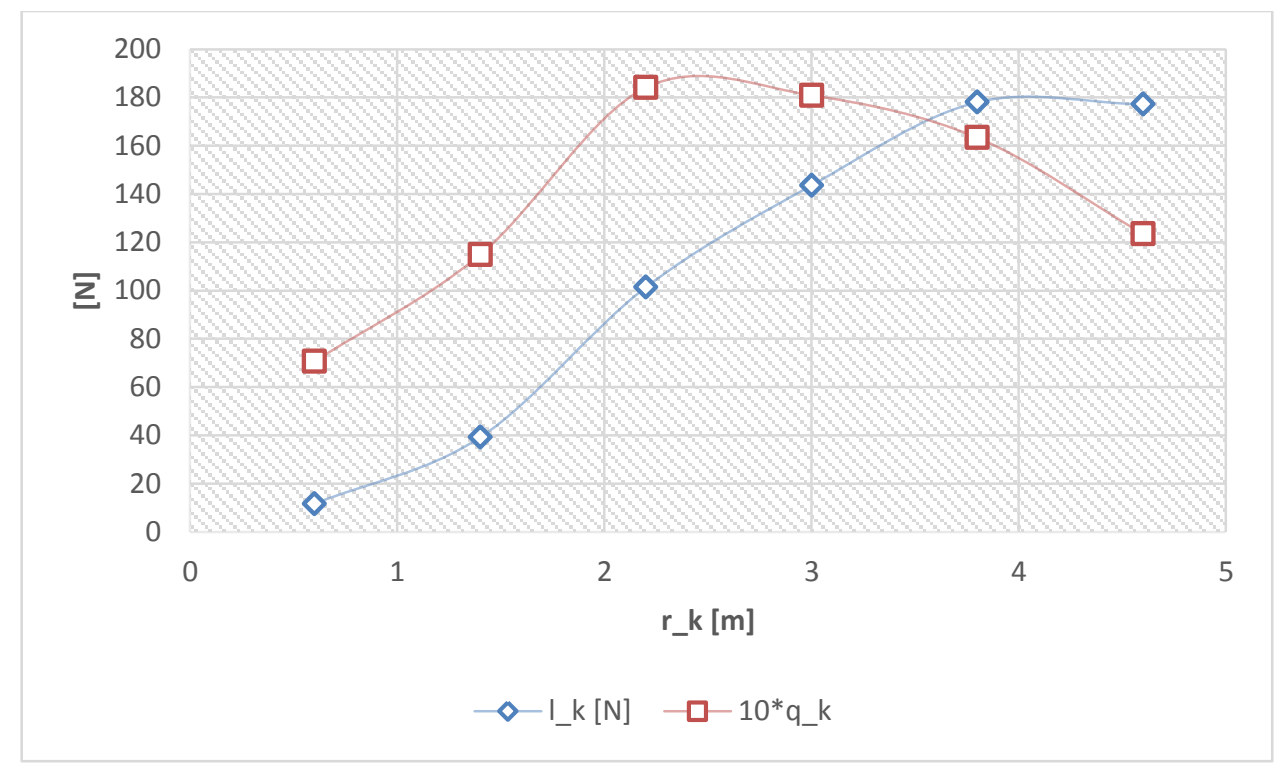

Figura 27. Representación gráfica de las distribuciones radiales de cargas $l_{k} y q_{k} \mathrm{rpm}=107, \mathrm{U}=7 \mathrm{~m} / \mathrm{s}$ 


\subsection{EJEMPLO DE IMPLEMENTACIÓN PRELIMINAR 2 [40]}

Con el objetivo de asegurar que el programa de cálculo no contiene errores se implementa otro ejemplo de rotor ya estudiado y con resultados publicados [40].

En este documento se estudian distintas geometrías de pala en las que las distribuciones de ángulos de paso geométrico y de cuerdas son constantes. Las características de la turbina en estudio son similares a las del apartado anterior, siendo el número de palas igual a 3 , el radio del extremo de pala 1 metro y el del núcleo de rotor es $0,1 \mathrm{~m}$.

La velocidad de la corriente es $U=2 \mathrm{~m} / \mathrm{s}$ y para la velocidad TSR se estudian varias posibilidades, una de ellas es TSR = 4 lo que implica que de acuerdo a la velocidad de la corriente $U$, implica que rpm = 76. El perfil bidimensional de las secciones de pala es constante siendo el correspondiente a la nomenclatura SG604 el escogido.

\begin{tabular}{|c|c|c|c|c|c|}
\hline no & $\mathbf{r}[\mathbf{k}][\mathbf{m}]$ & delta[k][m] & bheta[k][0] & $\mathbf{c}[\mathbf{k}][\mathbf{m}]$ & PERFIL \\
\hline 1 & 0,175 & 0,15 & 88 & 0,12 & SG6043 \\
\hline 2 & 0,325 & 0,15 & 88 & 0,12 & SG6043 \\
\hline 3 & 0,475 & 0,15 & 88 & 0,12 & SG6043 \\
\hline 4 & 0,625 & 0,15 & 88 & 0,12 & SG6043 \\
\hline 5 & 0,775 & 0,15 & 88 & 0,12 & SG6043 \\
\hline 6 & 0,925 & 0,15 & 88 & 0,12 & SG6043 \\
\hline
\end{tabular}

Tabla 7. Parámetros de geometría de pala implementados

La implementación en el método se realiza con un ángulo de paso geométrico igual a $12^{\circ}$, que con la referencia horizontal, el valor a introducir en el programa de cálculo será de $88^{\circ}$, y una cuerda constante a lo largo de la pala de 0,12 metros. Esta geometría se recoge en la siguiente Tabla 7.

Todos estos datos forman la entrada al programa que calcula la distribución de ángulos de ataque, así como las distribuciones de coeficientes de sustentación y coeficientes de arrastre que se muestran en la siguiente Tabla 8.

El coeficiente de potencia calculado es igual a 0,282 y la potencia del rotor es 3,6 kW.

\begin{tabular}{|c|c|c|c|c|c|c|c|c|}
\hline no & $\mathbf{r}[\mathbf{k}][\mathbf{m}]$ & delta[k][m] & bheta[k][0] & $\mathbf{c [ k ] [ m ]}$ & PERFIL & gamma[k][0] & cl[k] & cd[k] \\
\hline 1 & 0,175 & 0,15 & 88 & 0,12 & SG6043 & 50,27 & 0,513 & 0,762 \\
\hline 2 & 0,325 & 0,15 & 88 & 0,12 & SG6043 & 35,71 & 0,52 & 0,457 \\
\hline 3 & 0,475 & 0,15 & 88 & 0,12 & SG6043 & 25,88 & 0,625 & 0,273 \\
\hline 4 & 0,625 & 0,15 & 88 & 0,12 & SG6043 & 17,44 & 0,885 & 0,15 \\
\hline 5 & 0,775 & 0,15 & 88 & 0,12 & SG6043 & 10,78 & 1,567 & 0,05 \\
\hline 6 & 0,925 & 0,15 & 88 & 0,12 & SG6043 & 7,58 & 1,155 & 0,051 \\
\hline
\end{tabular}

Tabla 8. Distribuciones de ángulos de ataque, coeficientes de sustentación y arrastre, $r p m=76, U=2 \mathrm{~m} / \mathrm{s}$ 


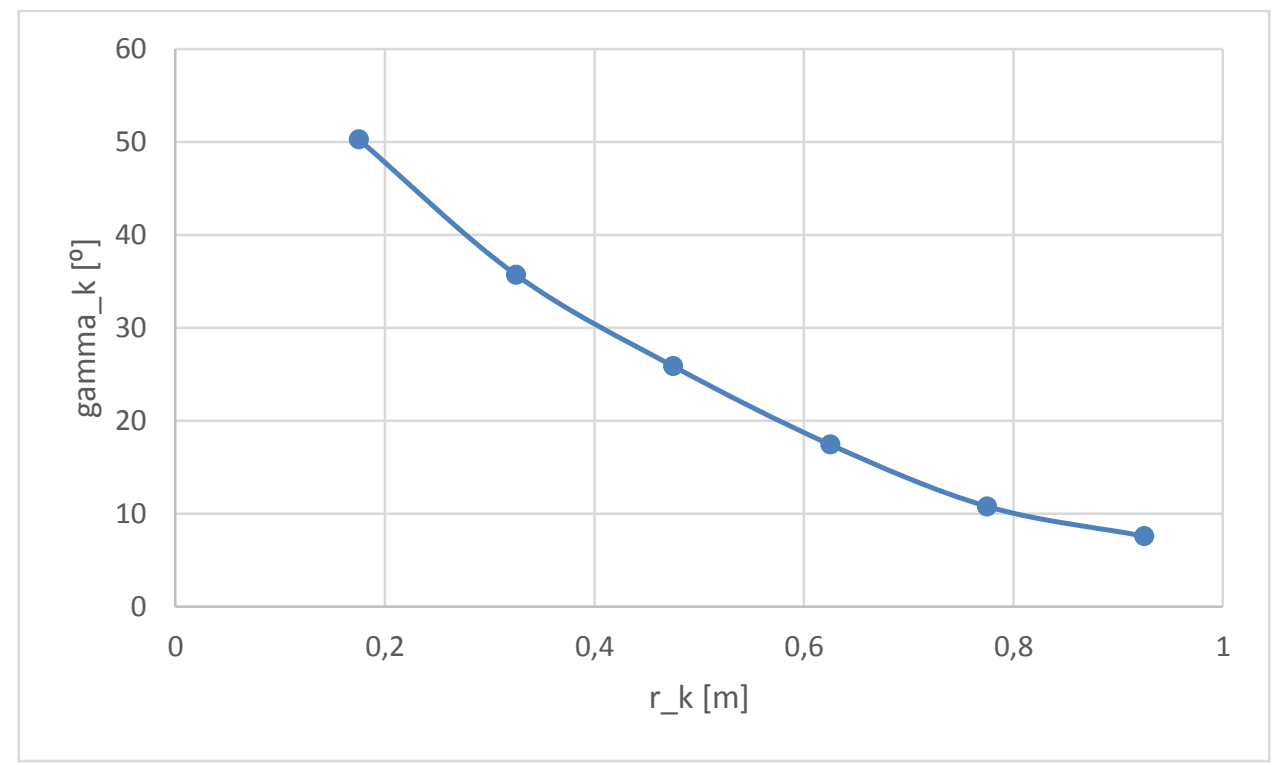

Figura 28. Representación gráfica de la distribución de ángulos de ataque $\gamma_{k} \mathrm{rpm}=76, \mathrm{U}=6 \mathrm{~m} / \mathrm{s}$

Puesto que este caso de implementación se ha realizado utilizando una geometría de pala sencilla, se trata de un buen ejemplo para ejecutar la rutina de cálculo que implementa el esquema del apartado 4.1.7.1.

En primer lugar hay que obtener el valor de las variables auxiliares $\lambda\left(\mathbf{r}_{\mathbf{k}}\right)$ y $\boldsymbol{\mu}\left(\mathbf{r}_{\mathbf{k}}\right)$, que se han de obtener con una herramienta de cálculo externa a la aplicación informática elaborada. En este caso se ha utilizado un fichero de cálculo de MAPLE. En la Figura 31, se representan gráficamente las distribuciones radiales de estas variables auxiliares.

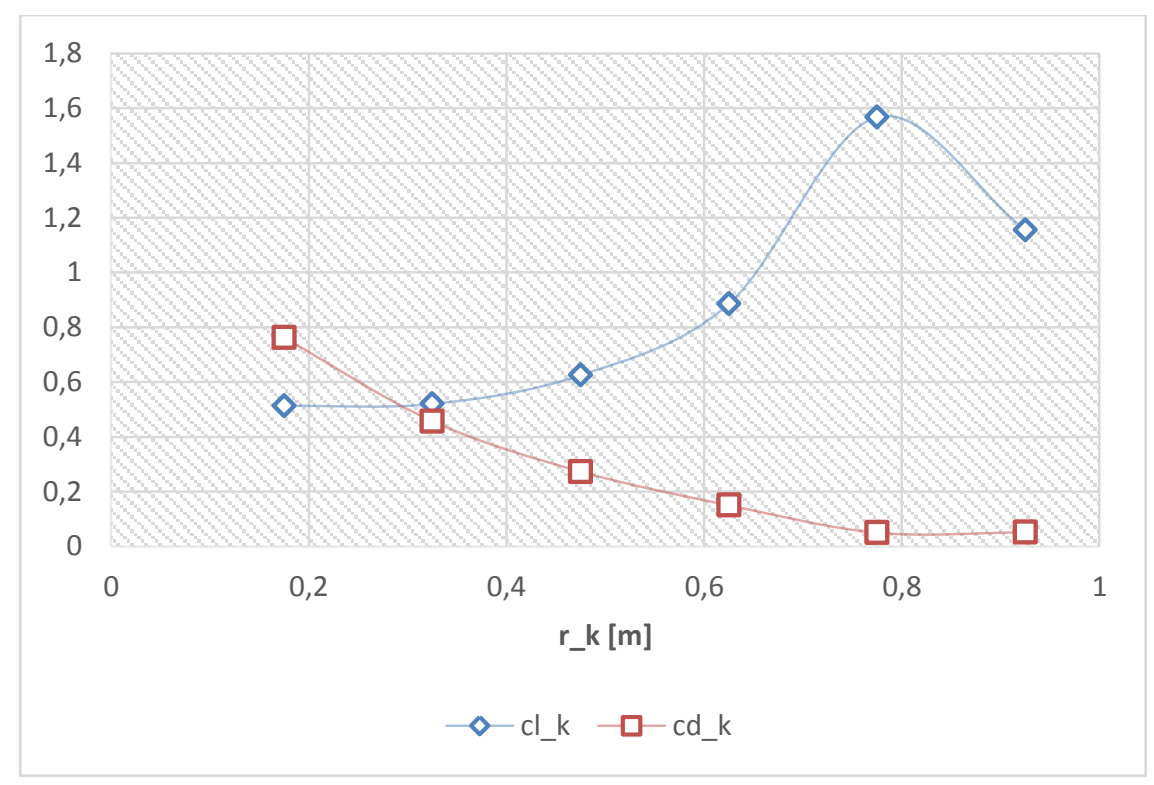

Figura 29. Representación gráfica de las distribuciones radiales de coeficientes de sustentación y arrastre $\mathrm{rpm}=76, \mathrm{U}=2 \mathrm{~m} / \mathrm{s}$ 


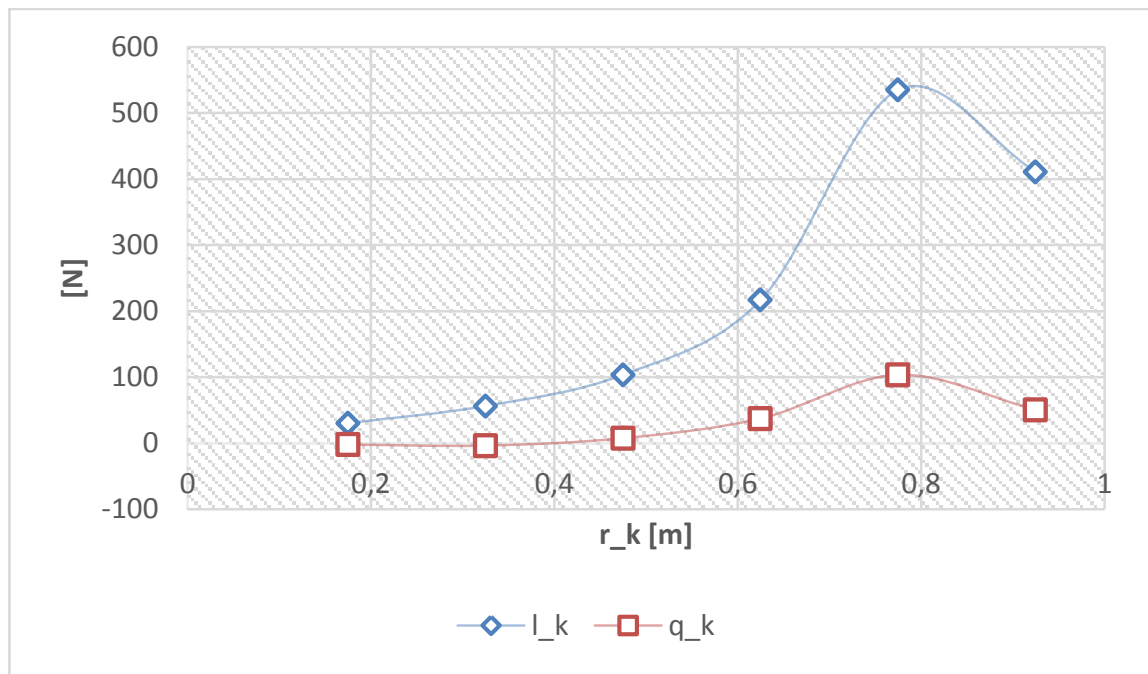

Figura 30. Representación gráfica de las distribuciones radiales de cargas $l_{k}$ y $q_{k} \mathrm{rpm}=76, \mathrm{U}=2 \mathrm{~m} / \mathrm{s}$

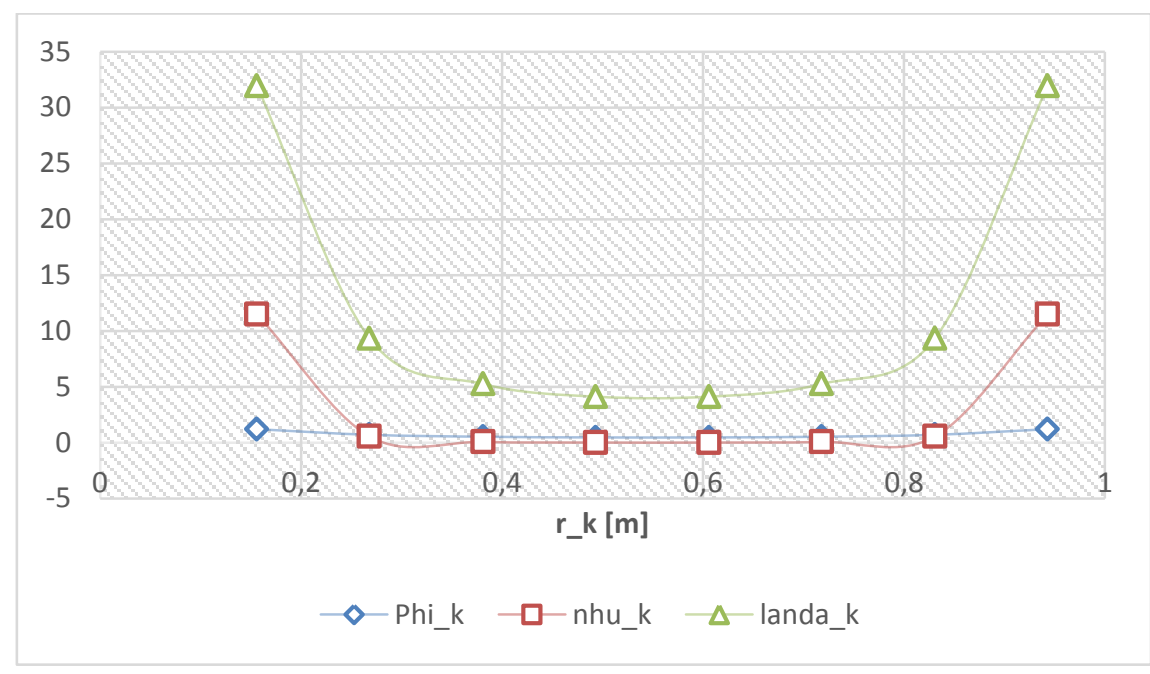

Figura 31. Representación gráfica de $\varphi_{k}, \lambda\left(r_{k}\right)$ y $\mu\left(r_{k}\right)$ con respecto al radio de cada sección de pala

Los parámetros de la geometría implementada, junto con las distribuciones de variables auxiliares, se recogen en la siguiente Tabla 9.

\begin{tabular}{|c|c|c|c|c|c|c|c|c|}
\hline no & r_k[m] & delta_k[m] & bheta_k[o] & c_k[m] & Phi_k & nhu_k & landa_k & PERFIL \\
\hline 1 & 0,156 & 0,112 & 88 & 0,12 & 1,2013 & 11,5069 & 31,96 & SG6043 \\
\hline 2 & 0,268 & 0,112 & 88 & 0,12 & 0,6976 & 0,5575 & 9,351 & SG6043 \\
\hline 3 & 0,381 & 0,112 & 88 & 0,12 & 0,524 & 0,0638 & 5,25 & SG6043 \\
\hline 4 & 0,493 & 0,112 & 88 & 0,12 & 0,4639 & 0,0043 & 4,111 & SG6043 \\
\hline 5 & 0,606 & 0,112 & 88 & 0,12 & 0,4639 & 0,0043 & 4,111 & SG6043 \\
\hline 6 & 0,718 & 0,112 & 88 & 0,12 & 0,524 & 0,0638 & 5,25 & SG6043 \\
\hline 7 & 0,831 & 0,112 & 88 & 0,12 & 0,6976 & 0,5575 & 9,351 & SG6043 \\
\hline 8 & 0,943 & 0,112 & 88 & 0,12 & 1,2013 & 11,5069 & 31,96 & SG6043 \\
\hline
\end{tabular}

Tabla 9. Geometría de pala implementada y variables auxiliares 
El coeficiente de potencia obtenido en base a las ecuaciones del procedimiento y a la geometría de pala de la tabla anterior sale negativo. Esto puede indicar que esta configuración de parámetros de geometría de pala no es compatible con las ecuaciones planteadas.

Pero esta compatibilidad se consigue si se disminuye el ángulo paso geométrico de pala a $70^{\circ}$, y con esta nueva configuración se calcula un coeficiente de potencia igual a 0,237 y una potencia de 3,05 $\mathrm{kW}$. La circulación es de $0,780 \mathrm{~m}^{2} / \mathrm{s}$ y la suma de las variables $\psi_{k}$ es de $0,022 \mathrm{~m}^{4} / \mathrm{s}^{2}$. Obteniéndose un régimen de funcionamiento de rotor equivalente al calculado según el método implementado basado en la Teoría de Elementos de Pala.

La distribución de ángulos de ataque $\gamma_{k}$, así como las distribuciones de coeficientes de sustentación y ángulos de ataque a lo largo de la pala se recogen en la Tabla 10.

\begin{tabular}{|c|c|c|c|c|c|c|c|c|}
\hline no & r_k[m] & delta_k[m] & bheta_k[0] & c_k[m] & PERFIL & gamma_k[0] & cl_k & cd_k \\
\hline 1 & 0,156 & 0,112 & 70 & 0,12 & SG6043 & 9,5 & 1,485 & 0,039 \\
\hline 2 & 0,268 & 0,112 & 70 & 0,12 & SG6043 & 8,28 & 1,389 & 0,036 \\
\hline 3 & 0,381 & 0,112 & 70 & 0,12 & SG6043 & 7,07 & 1,038 & 0,058 \\
\hline 4 & 0,493 & 0,112 & 70 & 0,12 & SG6043 & 5,85 & 0,885 & 0,059 \\
\hline 5 & 0,606 & 0,112 & 70 & 0,12 & SG6043 & 4,64 & 0,721 & 0,056 \\
\hline 6 & 0,718 & 0,112 & 70 & 0,12 & SG6043 & 3,42 & 0,567 & 0,051 \\
\hline 7 & 0,831 & 0,112 & 70 & 0,12 & SG6043 & 2,21 & 0,4 & 0,045 \\
\hline 8 & 0,943 & 0,112 & 70 & 0,12 & SG6043 & 1 & 0,245 & 0,038 \\
\hline
\end{tabular}

Tabla 10. Distribuciones de $\gamma_{k}$ para $r p m=76, U=2 \mathrm{~m} / \mathrm{s}$

Asimismo, si se implementa esta nueva geometría de pala en el método de cálculo de elementos de pala se obtiene un coeficiente de potencia negativo, al igual que se obtenía con la geometría inicial y la aplicación del procedimiento planteado en esta tesis. Por tanto, para comparar las cargas a las que está sometida la pala se tomarán como equivalentes la geometría inicial según el método de elementos de pala y la modificada según el procedimiento. De este modo se pueden trazar gráficas comparativas de estas cargas (Figura 32, Figura 33).

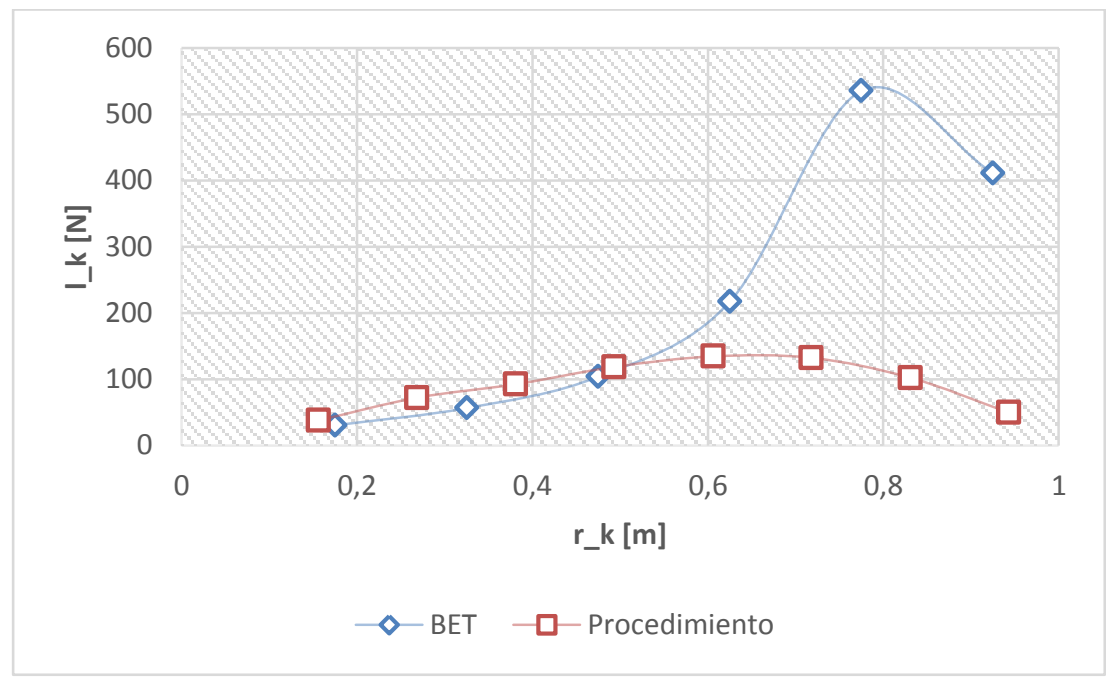

Figura 32. Gráfica comparativa de las cargas estáticas $l_{k}$ que se producen en la pala para $\mathrm{rpm}=76, \mathrm{U}=2 \mathrm{~m} / \mathrm{s}$ 


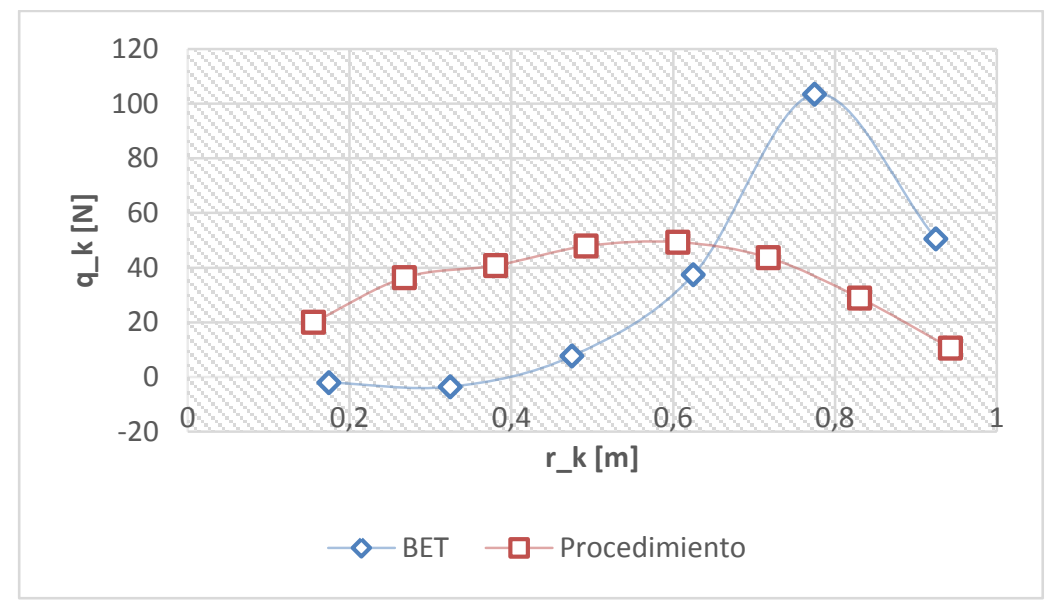

Figura 33. Gráfica comparativa de las cargas estáticas $q_{k}$ que se producen en la pala para rpm $=76, U=2 \mathrm{~m} / \mathrm{s}$

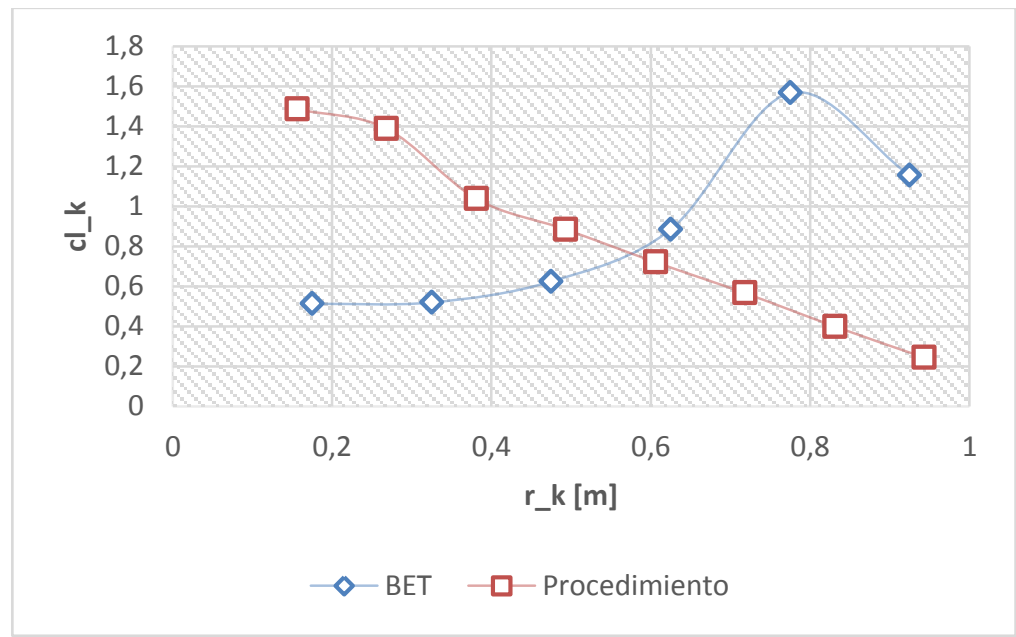

Figura 34. Representación gráfica de la distribución radial de coeficientes de sustentación para rpm $=76, \mathrm{U}=$ $2 \mathrm{~m} / \mathrm{s}$

\subsection{TURBINA DE 6,75 METROS DE DIÁMETRO DEL ROTOR [51]}

En este caso, se estudia una turbina de eje horizontal mediante elementos de pala, los datos principales de esta turbina se muestran en las siguientes tablas, en la Tabla 11, se representan los parámetros de funcionamiento del rotor, y en la Tabla 12 las características geométricas de la pala.

Con ellas se podrán definir los parámetros del rotor y la geometría de pala para los introducirlos en el procedimiento alternativo de cálculo, y en método de elementos de pala. Esta tarea se llevará a cabo con la ayuda de programas elaborados en lenguaje JAVA.

En la Tabla 12 están los datos relativos a la geometría de la pala. Empezando por la izquierda, primero aparecen los radios de cada una de las secciones y luego la distribución de cuerdas. En este caso, esta distribución se puede utilizar directamente, solamente con la salvedad de que el radio representativo de cada sección corresponde al radio inicial de la misma y no el medio como se vio en el apartado 2.7. 
Esta pequeña modificación resulta inmediata de subsanar. Adicionalmente se han elegido 9 secciones iguales para el estudio de esta turbina por lo que $\delta r_{k}=\frac{1}{3}$ metros. Y la ecuación de los radios de cada sección viene dada por,

$r_{k}=a+\delta r_{k}\left(k-\frac{1}{2}\right)=0,375+\frac{1}{3}\left(k-\frac{1}{2}\right), 1 \leq k \leq n=9$

\begin{tabular}{lc|c}
\hline Rotor diameter & $(\mathrm{m})$ & 6.75 \\
Blade Length & $(\mathrm{m})$ & 3 \\
Number of Blades & & 3 \\
Twist & $\left({ }^{\circ}\right)$ & 12 \\
Swept Area & $\left(\mathrm{m}^{2}\right)$ & 35.3 \\
Design Flow Speed & $(\mathrm{m} / \mathrm{s})$ & 1.7 \\
Design RPM & $(\mathrm{ppm})$ & 24 \\
Max Flow Speed & $(\mathrm{m} / \mathrm{s})$ & 2.5 \\
Max RPM & $(\mathrm{ppm})$ & 35.25 \\
Foil Type & & $\mathrm{NACA}$ \\
Design TSR & & $44 \mathrm{xx}$ \\
Power Coefficient & & 5 \\
\hline
\end{tabular}

Tabla 11. Parámetros principales del rotor [51]

\begin{tabular}{ccccccccc}
\hline \multicolumn{1}{l}{$\begin{array}{l}\text { length chord pitch } \\
(\mathrm{m})\end{array}$} & $\begin{array}{c}\text { inflow } \\
\text { angle }\end{array}$ & $\alpha\left(^{\circ}\right)$ & $R e$ & $\begin{array}{c}\text { NACA } \\
44 \mathrm{xx}\end{array}$ & $C_{L}$ & $C_{D}$ \\
\hline 0.6 & 500 & 16.5 & 26.4 & 9.9 & 1106100 & 4441 & 0.868 & 0.017 \\
0.9 & 480 & 11.0 & 20.9 & 9.9 & 1282165 & 4438 & 0.904 & 0.017 \\
1.2 & 460 & 7.0 & 16.8 & 9.8 & 1438808 & 4434 & 0.954 & 0.016 \\
1.5 & 430 & 5.5 & 13.5 & 8.0 & 1565234 & 4431 & 0.965 & 0.014 \\
1.8 & 390 & 5.0 & 10.2 & 5.2 & 1623068 & 4427 & 0.946 & 0.011 \\
2.1 & 330 & 4.5 & 9.9 & 5.4 & 1533421 & 4423 & 1.003 & 0.011 \\
2.4 & 260 & 4.5 & 9.9 & 5.4 & 1333776 & 4418 & 1.075 & 0.011 \\
2.7 & 200 & 4.5 & 9.4 & 4.9 & 1130972 & 4416 & 1.064 & 0.011 \\
3 & 180 & 4.5 & 9.3 & 4.8 & 1112795 & 4416 & 1.064 & 0.011 \\
\hline
\end{tabular}

Tabla 12. Características geométricas de la pala [51] 


\section{Ángulos de paso geométrico y perfiles sustentadores}

En el artículo [51] no se explica bien cuál es la referencia de los ángulos que aparecen en la Tabla 12 y cuál es su significado geométrico, por tanto, en adelante se propondrá una distribución de ángulos de ataque que puede ser bastante lógica para la geometría de la pala en estudio. Finalmente, El dato más complicado de adaptar para efectuar los cálculos es el del perfil sustentador.

En el modelo publicado se utiliza para cada sección un perfil diferente de la serie NACA44XX. Para preparar las distribuciones de los coeficientes de sustentación y arrastre no se ha encontrado [11] información acerca de todos los perfiles utilizados, solamente las relativas al NACA4418, por lo que se utilizarán los datos relativos al coeficiente de sustentación $c_{L}$ y de arrastre $c_{D}$ este perfil para todas las secciones de pala, suponiendo que la variación en los resultados va a ser pequeña.

Con todas estas consideraciones ya se tiene toda la información necesaria, recogida en las Tabla 13 y la Tabla 14, donde se representan los parámetros de funcionamiento principales del rotor, para los datos relativos a la geometría de la pala respectivamente.

\begin{tabular}{|c|c|}
\hline rho $[\mathrm{kg} / \mathrm{m} 3]$ & 1025,34 \\
\hline $\mathbf{U}[\mathrm{m} / \mathrm{s}]$ & 1,7 \\
\hline $\mathbf{H}[\mathrm{m}]$ & 17 \\
\hline $\mathbf{Z}$ & 3 \\
\hline $\mathbf{r p m}$ & 24 \\
\hline $\mathbf{a}[\mathrm{m}]$ & 0,375 \\
\hline $\mathbf{b}[\mathrm{m}]$ & 3,375 \\
\hline nhu $[\mathrm{m} 2 / \mathrm{s}]$ & 0,000001306 \\
\hline
\end{tabular}

Tabla 13. Parámetros principales de funcionamiento del rotor implementados

\begin{tabular}{|c|c|c|c|c|c|}
\hline SECTION no & $\boldsymbol{r}_{\boldsymbol{k}}[\boldsymbol{m}]$ & $\boldsymbol{\delta} \boldsymbol{r}_{\boldsymbol{k}}[\boldsymbol{m}]$ & $\boldsymbol{\beta}_{\boldsymbol{k}}\left[^{\circ}\right]$ & $\boldsymbol{c}_{\boldsymbol{k}}[\boldsymbol{m}]$ & Perfil \\
\hline 1 & 0,5415 & 0,333 & 73,5 & 0,500 & NACA 4418 \\
\hline 2 & 0,8745 & 0,333 & 79 & 0,480 & NACA 4418 \\
\hline 3 & 1,2075 & 0,333 & 83 & 0,460 & NACA 4418 \\
\hline 4 & 1,5405 & 0,333 & 84,5 & 0,430 & NACA 4418 \\
\hline 5 & 1,8735 & 0,333 & 85 & 0,390 & NACA 4418 \\
\hline 6 & 2,2065 & 0,333 & 85,5 & 0,330 & NACA 4418 \\
\hline 7 & 2,5395 & 0,333 & 85,5 & 0,260 & NACA 4418 \\
\hline 8 & 2,8725 & 0,333 & 85,5 & 0,200 & NACA 4418 \\
\hline 9 & 3,2055 & 0,333 & 85,5 & 0,180 & NACA 4418 \\
\hline
\end{tabular}

Tabla 14. Datos implementados de las características geométricas de la pala 


\section{Cálculo de las variables auxiliares}

Con estos datos de partida de la geometría de pala, se puede empezar con los cálculos, en el apartado 4.1.7.1, se muestra el esquema a seguir. Como se puede ver en este esquema, el primer paso consiste en obtener el valor de las variables auxiliares $\varphi_{k}, \lambda\left(r_{k}\right), \mu\left(r_{k}\right)$ y $V_{k}$.

En el caso de $\varphi_{\mathrm{k}}$, se calcula directamente en el programa de cálculo, mientras que para $\lambda\left(\mathrm{r}_{\mathrm{k}}\right)$ y $\mu\left(\mathrm{r}_{\mathrm{k}}\right)$ hay que hacer un paso intermedio, debido a que sus expresiones son integrales y requieren del uso de una herramienta adicional que permita resolver expresiones de este tipo. Para obtenerlos, se ha utilizado el fichero Cálculo de mu y lambda.mw de la aplicación informática MAPLE.

Estas dos variables $\left(\lambda\left(\mathrm{r}_{\mathrm{k}}\right)\right.$ y $\left.\mu\left(\mathrm{r}_{\mathrm{k}}\right)\right)$, una vez que se ha determinado el número de palas, el número de secciones de pala, los radios del núcleo y extremo de pala, no hay que volver a recalcularlas si se quiere hacer un estudio del comportamiento del rotor para distintas velocidades de rotación o de corriente de agua.

\begin{tabular}{|c|c|c|c|c|}
\hline SECTION no & $\boldsymbol{r}_{\boldsymbol{k}}[\boldsymbol{m}]$ & $\boldsymbol{\varphi}_{\boldsymbol{k}}$ & $\boldsymbol{\mu}\left(\boldsymbol{r}_{\boldsymbol{k}}\right)$ & $\boldsymbol{\lambda}\left(\boldsymbol{r}_{\boldsymbol{k}}\right)$ \\
\hline 1 & 0,5415 & 1,30750 & 4,7703 & 10,927 \\
\hline 2 & 0,8745 & 0,82204 & 0,32432 & 3,4884 \\
\hline 3 & 1,2075 & 0,61999 & 0,04614 & 1,9616 \\
\hline 4 & 1,5405 & 0,53587 & 0,00649 & 1,4634 \\
\hline 5 & 1,8735 & 0,51211 & $-0,001$ & 1,335 \\
\hline 6 & 2,2065 & 0,53543 & 0,00641 & 1,4609 \\
\hline 7 & 2,5395 & 0,61885 & 0,04547 & 1,9544 \\
\hline 8 & 2,8725 & 0,81931 & 0,31794 & 3,4644 \\
\hline 9 & 3,2055 & 1,30118 & 4,7703 & 10,744 \\
\hline
\end{tabular}

Tabla 15. Valores de las variables auxiliares $\varphi_{\mathrm{k}}, \lambda\left(\mathbf{r}_{\mathrm{k}}\right)$ y $\mu\left(\mathbf{r}_{\mathrm{k}}\right)$

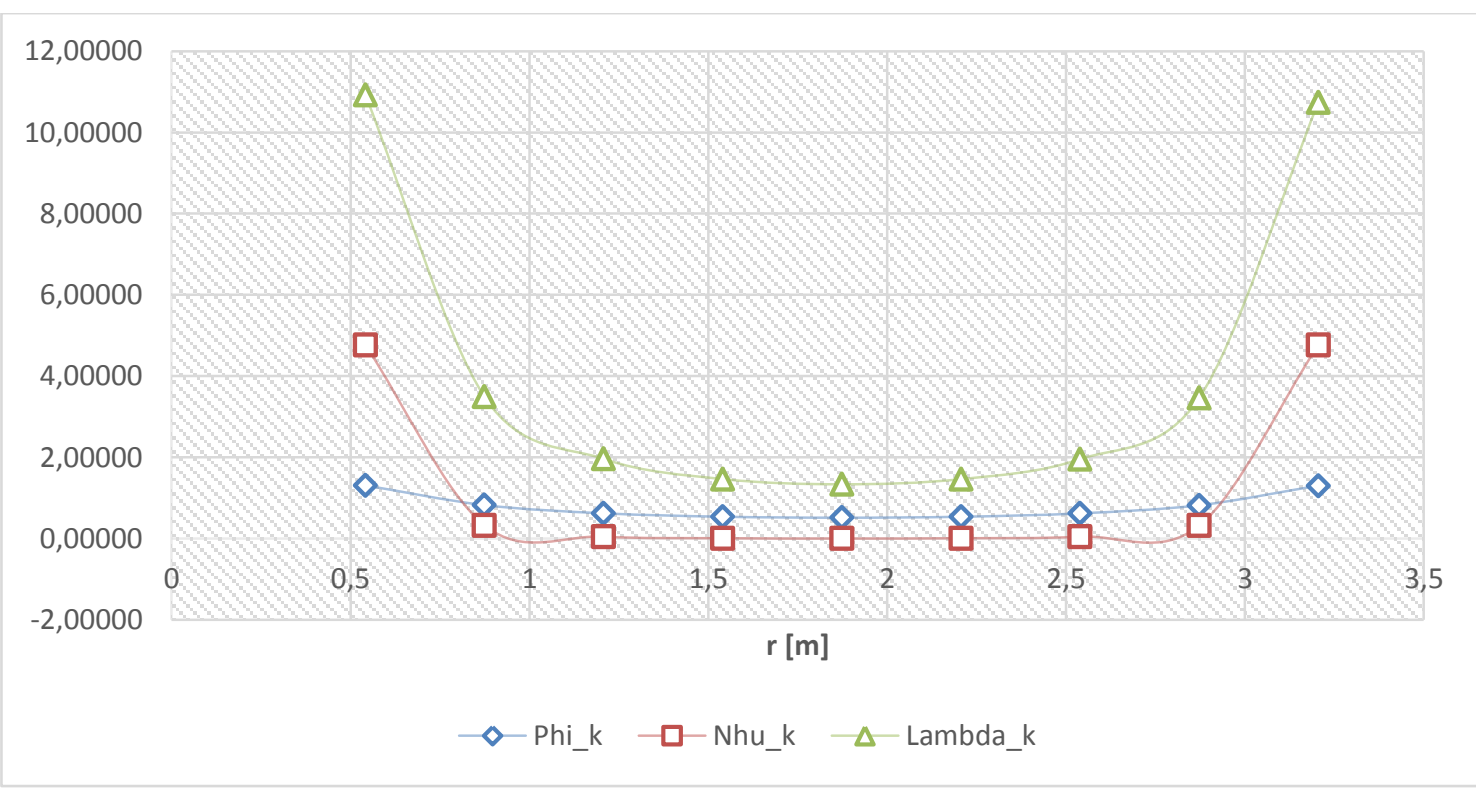

Figura 35. Representación gráfica de $\varphi_{k}, \lambda\left(r_{k}\right)$ y $\mu\left(r_{k}\right)$ con respecto al radio de cada sección de pala 
Cálculo de los ángulos de ataque $\gamma_{k}$, coeficiente de potencia $c_{P}$ y circulación $\Gamma$ para la velocidad de rotación nominal $(\mathrm{rpm}=24)$

Con los parámetros de geometría de pala que se muestran en la Tabla 14, mediante varias iteraciones de cálculo, se obtiene la distribución de ángulos de ataque $\gamma_{\mathrm{k}}$ que hace que la suma de las variables auxiliares $\psi_{\mathrm{k}}$ sea aproximadamente cero, y que por tanto, se pueden dar como solución de las ecuaciones que se plantean en este procedimiento de cálculo, se muestra en la Tabla 17.

Esta distribución de ángulos de ataque, origina que la suma total de las variables auxiliares $\psi_{k}$ tenga un valor igual a -0,048 $\mathrm{m} 4 / \mathrm{s} 2$, que es muy cercano a cero y que confirma que esta distribución de $\gamma_{\mathrm{k}}$ es solución aproximada del sistema de ecuaciones que componen el procedimiento alternativo.

En cuanto la circulación $\Gamma$ el valor obtenido es de 1,635 m2/s, y finalmente el coeficiente de potencia $c_{P}$ es de 0,063 .

Como se puede observar el coeficiente de potencia está muy por debajo del valor esperado, cercano a 0,5 .

\begin{tabular}{|c|c|c|c|c|c|c|c|c|}
\hline no & r_k[m] & delta_k[m] & bheta_k[0] & c_k[m] & PERFIL & gamma_k[0] & cl_k & cd_k \\
\hline 1 & 0,542 & 0,333 & 73,5 & 0,5 & NACA4418 & $\mathbf{5 , 1}$ & 1,037 & 0,009 \\
\hline 2 & 0,875 & 0,333 & 79 & 0,48 & NACA4418 & $\mathbf{4 , 0 2}$ & 0,911 & 0,008 \\
\hline 3 & 1,208 & 0,333 & 83 & 0,46 & NACA4418 & $\mathbf{2 , 9 5}$ & 0,787 & 0,007 \\
\hline 4 & 1,541 & 0,333 & 84,5 & 0,43 & NACA4418 & $\mathbf{1 , 8 7}$ & 0,678 & 0,007 \\
\hline 5 & 1,874 & 0,333 & 85 & 0,39 & NACA4418 & $\mathbf{0 , 7 9}$ & 0,565 & 0,007 \\
\hline 6 & 2,206 & 0,333 & 85,5 & 0,33 & NACA4418 & $\mathbf{- 0 , 2 7}$ & 0,45 & 0,008 \\
\hline 7 & 2,54 & 0,333 & 85,5 & 0,26 & NACA4418 & $\mathbf{- 1 , 3 4}$ & 0,332 & 0,008 \\
\hline 8 & 2,873 & 0,333 & 85,5 & 0,2 & NACA4418 & $\mathbf{- 2 , 4 2}$ & 0,215 & 0,008 \\
\hline 9 & 3,206 & 0,333 & 85,5 & 0,18 & NACA4418 & $\mathbf{- 3 , 5}$ & 0,096 & 0,008 \\
\hline
\end{tabular}

Tabla 16. Distribuciones de $\gamma_{k}$ para $r p m=24, U=1,7 \mathrm{~m} / \mathrm{s}$

Cálculo de los ángulos de ataque $\gamma_{k}$, coeficiente de potencia $c_{P}$ y circulación $\Gamma$ para la velocidad de rotación nominal ( $r p m=24)$ mediante implementación del método iterativo de elementos de pala.

El método iterativo de elementos de pala se ha implementado, al igual que el procedimiento alternativo, en una aplicación confeccionada con leguaje JAVA. En ambas aplicaciones el núcleo del programa es el mismo en cuanto a lectura de datos de ficheros y estructura global, aunque como es obvio, la rutina de cálculos cambia.

Así pues se ejecuta el programa con la geometría de pala de la Tabla 14, y se obtienen los resultados que se reflejan en la siguiente Tabla 16. 


\begin{tabular}{|c|c|c|c|c|c|c|c|c|}
\hline $\mathrm{n} \mathbf{0}$ & $\mathrm{r}[\mathrm{k}][\mathrm{m}]$ & delta[k][m] & bheta[k][] & $\mathrm{c}[\mathrm{k}][\mathrm{m}]$ & PERFIL & gamma[k][] & $\mathrm{cl}[\mathrm{k}]$ & $\mathrm{cd}[\mathrm{k}]$ \\
\hline 1 & 0,542 & 0,333 & $\mathbf{7 3 , 5}$ & 0,5 & NACA4418 & $\mathbf{2 9 , 5 3}$ & 0,805 & 0,261 \\
\hline 2 & 0,875 & 0,333 & 79 & 0,48 & NACA4418 & $\mathbf{2 1 , 8 9}$ & 1,071 & 0,132 \\
\hline 3 & 1,208 & 0,333 & 83 & 0,46 & NACA4418 & $\mathbf{1 3 , 7 4}$ & 1,585 & 0,031 \\
\hline 4 & 1,541 & 0,333 & 84,5 & 0,43 & NACA4418 & $\mathbf{9 , 9 4}$ & 1,415 & 0,015 \\
\hline 5 & 1,874 & 0,333 & 85 & 0,39 & NACA4418 & $\mathbf{7 , 9 7}$ & 1,273 & 0,011 \\
\hline 6 & 2,206 & 0,333 & 85,5 & 0,33 & NACA4418 & $\mathbf{8 , 4 1}$ & 1,308 & 0,012 \\
\hline 7 & 2,54 & 0,333 & 85,5 & 0,26 & NACA4418 & $\mathbf{7 , 2 9}$ & 1,219 & 0,01 \\
\hline 8 & 2,873 & 0,333 & 85,5 & 0,2 & NACA4418 & $\mathbf{6 , 4 5}$ & 1,148 & 0,009 \\
\hline 9 & 3,206 & 0,333 & 85,5 & 0,18 & NACA4418 & $\mathbf{5 , 4 9}$ & 1,068 & 0,009 \\
\hline
\end{tabular}

Tabla 17. Distribuciones de $\gamma_{\mathrm{k}} \mathrm{rpm}=24$ con el método de elementos de pala [20]

El coeficiente de potencia obtenido bastante aproximado al obtenido en la publicación, siendo $c_{P}=0,521$ y la potencia $P=47 \mathrm{~kW}$.

En la Figura 36 se muestra gráfica comparativa de las distribuciones de coeficientes de sustentación obtenidas tanto con la implementación directa del método de elementos de pala y los coeficientes de sustentación calculados con el procedimiento propuesto en esta tesis.

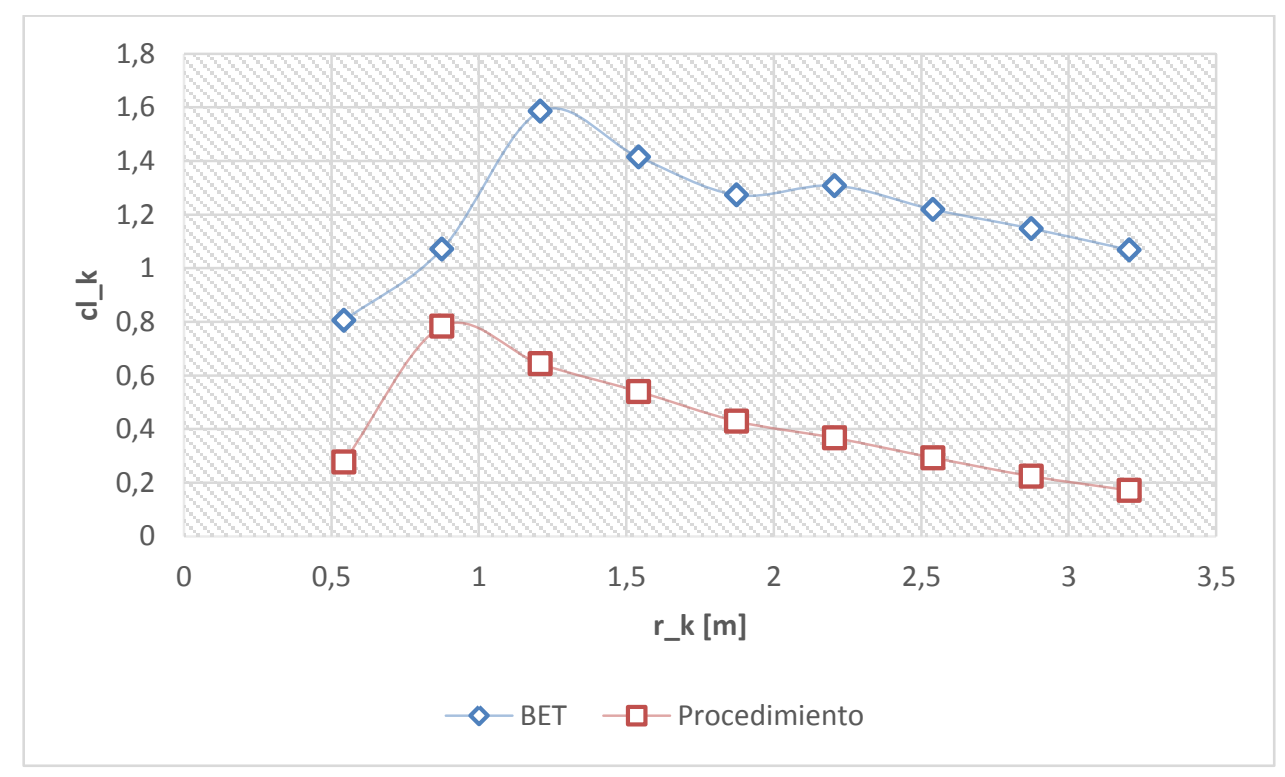

Figura 36. Representación gráfica de la distribución radial de coeficientes de sustentación para $\mathrm{rpm}=\mathbf{2 4}, \mathrm{U}=$ $1,7 \mathrm{~m} / \mathrm{s}$

\section{Modificación de geometría de pala}

Utilizando como base la geometría de pala implementada anteriormente, se va a efectuar una ligera rotación de las secciones de pala, con el objetivo de obtener mejores resultados, sobre todo en cuanto a al coeficiente de potencia.

La rotación de las secciones de pala se va a tantear restando intervalos de 5 ó 3 grados en la distribución de ángulos de paso geométrico, que de diversas pruebas preliminares efectuadas se ha comprobado que los resultados de la implementación del procedimiento mejoran al estar algunos 
grados las distribuciones de pala, sobre todo si éstas tienen ángulos cercanos a $90^{\circ}$ en el extremo de pala.

Como se ha dicho, al restar algunos grados a las secciones de ángulo de paso geométrico se mejoran considerablemente los resultados, y finalmente se elige un intervalo de 8 grados con respecto a la distribución inicial mostrada en la Tabla 14.

Con esta nueva distribución de ángulos de paso geométrico implementada en el procedimiento alternativo, se obtiene un coeficiente de potencia igual a 0,313 y la potencia de rotor obtenida es de $28,23 \mathrm{~kW}$. La circulación $\Gamma$ es $2,260 \mathrm{~m}^{2} / \mathrm{s}$ y la suma de las variables auxiliares $\psi_{k}$ es de $-0,056 \mathrm{~m}^{4} / \mathrm{s}^{2}$.

La distribución de ángulos de ataque $\gamma_{k}$, según el procedimiento alternativo se muestra en la Tabla 18.

\begin{tabular}{|c|c|c|c|c|c|c|c|c|}
\hline no & r_k[m] & delta_k[m] & bheta_k[o] & c_k[m] & PERFIL & gamma_k[0] & cl_k & cd_k \\
\hline 1 & 0,542 & 0,333 & 65,5 & 0,5 & NACA4418 & $\mathbf{6 , 1}$ & 1,118 & 0,009 \\
\hline 2 & 0,875 & 0,333 & 71 & 0,48 & NACA4418 & $\mathbf{5 , 2 7}$ & 1,05 & 0,009 \\
\hline 3 & 1,208 & 0,333 & 75 & 0,46 & NACA4418 & $\mathbf{4 , 4 5}$ & 0,976 & 0,008 \\
\hline 4 & 1,541 & 0,333 & 76,5 & 0,43 & NACA4418 & $\mathbf{3 , 6 2}$ & 0,856 & 0,008 \\
\hline 5 & 1,874 & 0,333 & 77 & 0,39 & NACA4418 & $\mathbf{2 , 8}$ & 0,772 & 0,007 \\
\hline 6 & 2,206 & 0,333 & 77,5 & 0,33 & NACA4418 & $\mathbf{1 , 9 7}$ & 0,689 & 0,007 \\
\hline 7 & 2,54 & 0,333 & 77,5 & 0,26 & NACA4418 & $\mathbf{1 , 1 5}$ & 0,602 & 0,007 \\
\hline 8 & 2,873 & 0,333 & 77,5 & 0,2 & NACA4418 & $\mathbf{0 , 3 2}$ & 0,514 & 0,008 \\
\hline 9 & 3,206 & 0,333 & 77,5 & 0,18 & NACA4418 & $\mathbf{- 0 , 4 9}$ & 0,425 & 0,008 \\
\hline
\end{tabular}

Tabla 18. Nueva geometría de pala implementada y ángulos de ataque $\gamma_{k}$ obtenidos con el procedimiento alternativo

Si se implementa esta nueva geometría de pala en el método de elementos de pala se obtiene un coeficiente de potencia igual a 0,341 y una potencia de rotor de 30,70 kW. En la Tabla 19 se recoge la distribución de ángulos de ataque y coeficientes de sustentación y arrastre.

\begin{tabular}{|c|c|c|c|c|c|c|c|c|}
\hline $\mathrm{n} \mathbf{0}$ & $\mathrm{r}[\mathrm{k}][\mathrm{m}]$ & delta $[\mathrm{k}][\mathrm{m}]$ & bheta[k][] & $\mathrm{c}[\mathrm{k}][\mathrm{m}]$ & PERFIL & gamma[k][] & $\mathrm{cl}[\mathrm{k}]$ & $\mathrm{cd}[\mathrm{k}]$ \\
\hline 1 & 0,542 & 0,333 & 65,5 & 0,5 & NACA4418 & $\mathbf{1 1 , 1 7}$ & 1,486 & 0,018 \\
\hline $\mathbf{2}$ & 0,875 & 0,333 & 71 & 0,48 & NACA4418 & $\mathbf{8 , 2 3}$ & 1,294 & 0,012 \\
\hline 3 & 1,208 & 0,333 & 75 & 0,46 & NACA4418 & $\mathbf{7 , 5 6}$ & 1,242 & 0,011 \\
\hline 4 & 1,541 & 0,333 & 76,5 & 0,43 & NACA4418 & $\mathbf{4 , 8 6}$ & 1,019 & 0,008 \\
\hline 5 & 1,874 & 0,333 & 77 & 0,39 & NACA4418 & $\mathbf{2 , 8 5}$ & 0,778 & 0,007 \\
\hline 6 & 2,206 & 0,333 & 77,5 & 0,33 & NACA4418 & $\mathbf{1 , 6 6}$ & 0,656 & 0,007 \\
\hline 7 & 2,54 & 0,333 & 77,5 & 0,26 & NACA4418 & $\mathbf{0 , 6 4}$ & 0,548 & 0,007 \\
\hline 8 & 2,873 & 0,333 & 77,5 & 0,2 & NACA4418 & $\mathbf{0 , 7 4}$ & 0,559 & 0,007 \\
\hline 9 & 3,206 & 0,333 & 77,5 & 0,18 & NACA4418 & $\mathbf{- 0 , 5 8}$ & 0,416 & 0,008 \\
\hline
\end{tabular}

Tabla 19. Nueva geometría de pala implementada y ángulos de ataque $\gamma_{k}$ obtenidos con el método de elementos de pala $\mathrm{rpm}=24, \mathrm{U}=1,7 \mathrm{~m} / \mathrm{s}$. 
En la Figura 37, se comparan las cargas estáticas $l_{k}$, obtenidas por ambos métodos de cálculo, que tiene que soportar la estructura de la pala. Y en la Figura 38, se ha representado la distribución de cargas $q_{k}$ a lo largo de la pala.

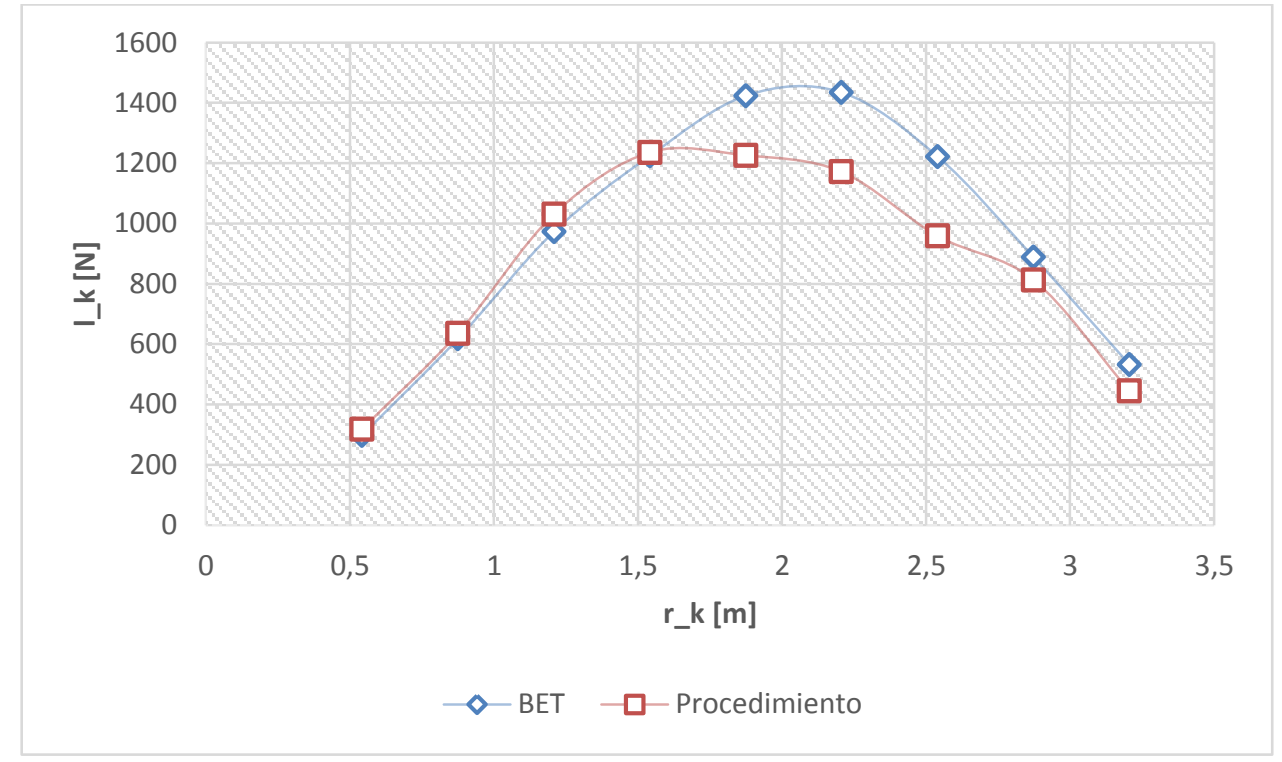

Figura 37. Gráfica comparativa de las cargas estáticas $l_{k}$ que se producen en la pala para $r p m=24, U=1,7$ $\mathrm{m} / \mathrm{s}$

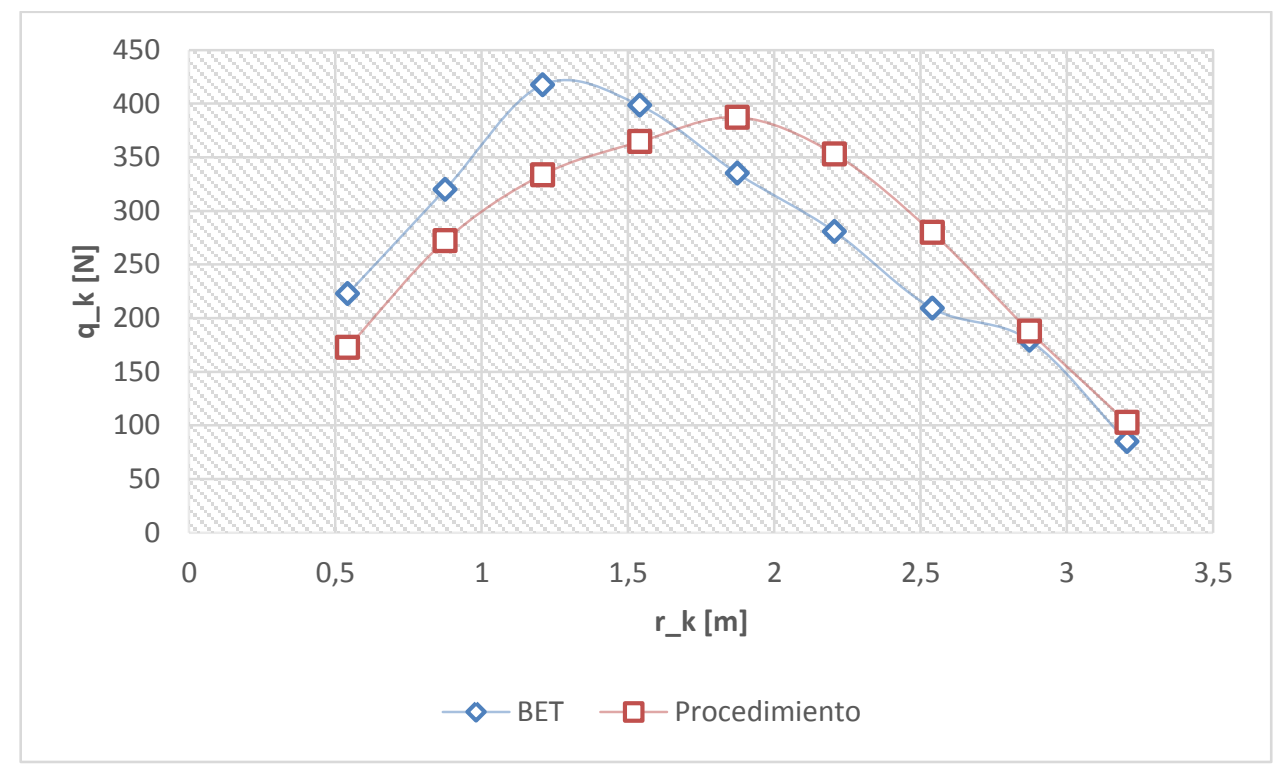

Figura 38. Gráfica comparativa de las cargas $q_{k}$ que se producen en la pala para $\mathrm{rpm}=\mathbf{2 4 ,} \mathrm{U}=\mathbf{1 , 7} \mathrm{m} / \mathrm{s}$

\section{Comportamiento del rotor a distintas TSR}

Con las condiciones de funcionamiento de rotor con $20 \mathrm{rpm}$ y $1,7 \mathrm{~m} / \mathrm{s}$ de velocidad de la corriente, con el procedimiento de cálculo se obtiene un coeficiente de potencia igual a 0,187 , una potencia de 
rotor de $16,93 \mathrm{~kW}$, la circulación es $2,012 \mathrm{~m}^{2} / \mathrm{s}$ y la suma de las variables auxiliares $\psi_{k}$ es igual a 0,008 .

Las distribuciones de ángulos de ataque coeficientes de sustentación y coeficientes de arrastre se recogen en la siguiente Tabla 20.

\begin{tabular}{|c|c|c|c|c|c|c|c|c|}
\hline no & r_k[m] & delta_k[m] & bheta_k[o] & c_k[m] & PERFIL & gamma_k[0] & cl_k & cd_k \\
\hline 1 & 0,542 & 0,333 & 65,5 & 0,5 & NACA4418 & 6 & 1,11 & 0,009 \\
\hline 2 & 0,875 & 0,333 & 71 & 0,48 & NACA4418 & 5,17 & 1,042 & 0,009 \\
\hline 3 & 1,208 & 0,333 & 75 & 0,46 & NACA4418 & 4,35 & 0,961 & 0,008 \\
\hline 4 & 1,541 & 0,333 & 76,5 & 0,43 & NACA4418 & 3,52 & 0,843 & 0,008 \\
\hline 5 & 1,874 & 0,333 & 77 & 0,39 & NACA4418 & 2,7 & 0,763 & 0,007 \\
\hline 6 & 2,206 & 0,333 & 77,5 & 0,33 & NACA4418 & 1,87 & 0,678 & 0,007 \\
\hline 7 & 2,54 & 0,333 & 77,5 & 0,26 & NACA4418 & 1,05 & 0,591 & 0,007 \\
\hline 8 & 2,873 & 0,333 & 77,5 & 0,2 & NACA4418 & 0,22 & 0,504 & 0,008 \\
\hline 9 & 3,206 & 0,333 & 77,5 & 0,18 & NACA4418 & $-0,59$ & 0,414 & 0,008 \\
\hline
\end{tabular}

Tabla 20. Nueva geometría de pala implementada y ángulos de ataque $\gamma_{k}$ obtenidos con el procedimiento pala rpm $=20, U=1,7 \mathrm{~m} / \mathrm{s}$.

El coeficiente de potencia con esta configuración de funcionamiento de rotor obtenido de acuerdo al método de elementos de pala es igual a 0,335 y la potencia de rotor es 30,24 kW. En la siguiente Tabla 21 se recogen las distribuciones de ángulos de ataque, coeficientes de sustentación y coeficientes de arrastre.

\begin{tabular}{|c|c|c|c|c|c|c|c|c|}
\hline $\mathbf{n} \mathbf{0}$ & $\mathbf{r}[\mathbf{k}][\mathbf{m}]$ & delta[k][m] & bheta[k][0] & $\mathbf{c}[\mathbf{k}][\mathbf{m}]$ & PERFIL & gamma[k][] & cl[k] & $\mathbf{c d}[\mathbf{k}]$ \\
\hline 1 & 0,542 & 0,333 & 65,5 & 0,5 & NACA4418 & 23,56 & 0,996 & 0,158 \\
\hline 2 & 0,875 & 0,333 & 71 & 0,48 & NACA4418 & 12,92 & 1,563 & 0,026 \\
\hline 3 & 1,208 & 0,333 & 75 & 0,46 & NACA4418 & 11,11 & 1,482 & 0,018 \\
\hline 4 & 1,541 & 0,333 & 76,5 & 0,43 & NACA4418 & 8,92 & 1,346 & 0,013 \\
\hline 5 & 1,874 & 0,333 & 77 & 0,39 & NACA4418 & 6,14 & 1,122 & 0,009 \\
\hline 6 & 2,206 & 0,333 & 77,5 & 0,33 & NACA4418 & 4,54 & 0,987 & 0,008 \\
\hline 7 & 2,54 & 0,333 & 77,5 & 0,26 & NACA4418 & 3,09 & 0,8 & 0,007 \\
\hline 8 & 2,873 & 0,333 & 77,5 & 0,2 & NACA4418 & 3,27 & 0,816 & 0,007 \\
\hline 9 & 3,206 & 0,333 & 77,5 & 0,18 & NACA4418 & 1,7 & 0,661 & 0,007 \\
\hline
\end{tabular}

Tabla 21. Nueva geometría de pala implementada y ángulos de ataque $\gamma_{k}$ obtenidos con el método de elementos de pala para $\mathrm{rpm}=20, \mathrm{U}=1,7 \mathrm{~m} / \mathrm{s}$.

Bajando la velocidad de rotación del rotor a $12 \mathrm{rpm}$ y manteniendo $U=1,7 \mathrm{~m} / \mathrm{s}(T S R=7,392)$, se recalculan los parámetros de funcionamiento del rotor con el esquema de cálculos del procedimiento propuesto en esta tesis.

Con esta velocidad de rotación se obtiene un coeficiente de potencia igual a 0,044 y una potencia de rotor de 3,9 kW. La circulación es de $1,612 \mathrm{~m}^{2} / \mathrm{s}$ y la suma de las variables auxiliares $\psi_{k}$ es igual a $0,089 \mathrm{~m}^{4} / \mathrm{s}^{2}$. 
Con estas condiciones de funcionamiento de rotor, se ejecuta el método de cálculo de elementos de pala y, en este caso, con la misma geometría de pala y se obtiene un coeficiente de potencia igual a 0,136 y una potencia de rotor de $12,32 \mathrm{~kW}$.

\subsection{TURBINA DE 20 METROS DE DIÁMETRO [28]}

En este otro caso, se trata de un buen ejemplo para comprobar resultados, ya que en este documento [28] aportan información detallada de la geometría de pala y los cálculos se han efectuado con la técnica de CFD, por lo que se espera que sean de mayor precisión que los de la publicación de la turbina estudiada en el apartado anterior, en la que se aplicó directamente la técnica de elementos de pala (BEM).

En la Tabla 22 se recogen los parámetros principales de la turbina, éstos son perfectamente identificables para el procedimiento de cálculo, exceptuando el dato del radio del núcleo del rotor $a$, que, en este caso, se estimará como igual a 1 metro.

\begin{tabular}{lc}
\hline Design Parameters & Values \\
\hline $\mathrm{P}_{\text {rated }}$ : Rated Power & $1 \mathrm{MW}$ \\
$\mathrm{C}_{\mathrm{p}}$ : Estimated power coefficient & 0.46 \\
$\mathrm{~V}_{\text {rated }}$ : Rated current velocity & $2.4 \mathrm{~m} / \mathrm{s}$ \\
$\rho:$ Sea water density & $1024 \mathrm{~kg} / \mathrm{m}^{3}$ \\
$\lambda$ : Tip speed ratio & 6 \\
D: Diameter & $20 \mathrm{~m}$ \\
N: Blade number & 3 \\
$\omega:$ Rotational speed & $11.5 \mathrm{~min}^{-1}$
\end{tabular}

Tabla 22. Parámetros principales de funcionamiento del rotor [28]

\begin{tabular}{|c|c|}
\hline rho $[\mathrm{kg} / \mathrm{m} 3]$ & 1025,34 \\
\hline $\mathbf{U}[\mathrm{m} / \mathrm{s}]$ & 2,4 \\
\hline $\mathbf{H}[\mathrm{m}]$ & 17 \\
\hline $\mathbf{Z}$ & 3 \\
\hline $\mathbf{r p m}$ & 11,5 \\
\hline $\mathbf{a}[\mathrm{m}]$ & 1 \\
\hline $\mathbf{b}[\mathrm{m}]$ & 10 \\
\hline nhu $[\mathrm{m} 2 / \mathrm{s}]$ & 0,000001306 \\
\hline
\end{tabular}

Tabla 23. Parámetros de funcionamiento del rotor en el procedimiento de cálculo

Una vez que se tienen los parámetros principales del rotor (Tabla 23), hay que definir la geometría de la pala. En el documento se muestran las distribuciones de cuerdas y ángulos de ataque, éstas se 
muestran en la Figura 39. En ella se puede ver que la cuerda alcanza su máximo para un radio de aproximadamente 2 metros y luego va decreciendo hasta 0,2 metros en el extremo de la pala.

Los ángulos de ataque geométricos se mantienen constantes en los radios cercanos al núcleo del rotor y luego va decreciendo a lo largo de la pala.

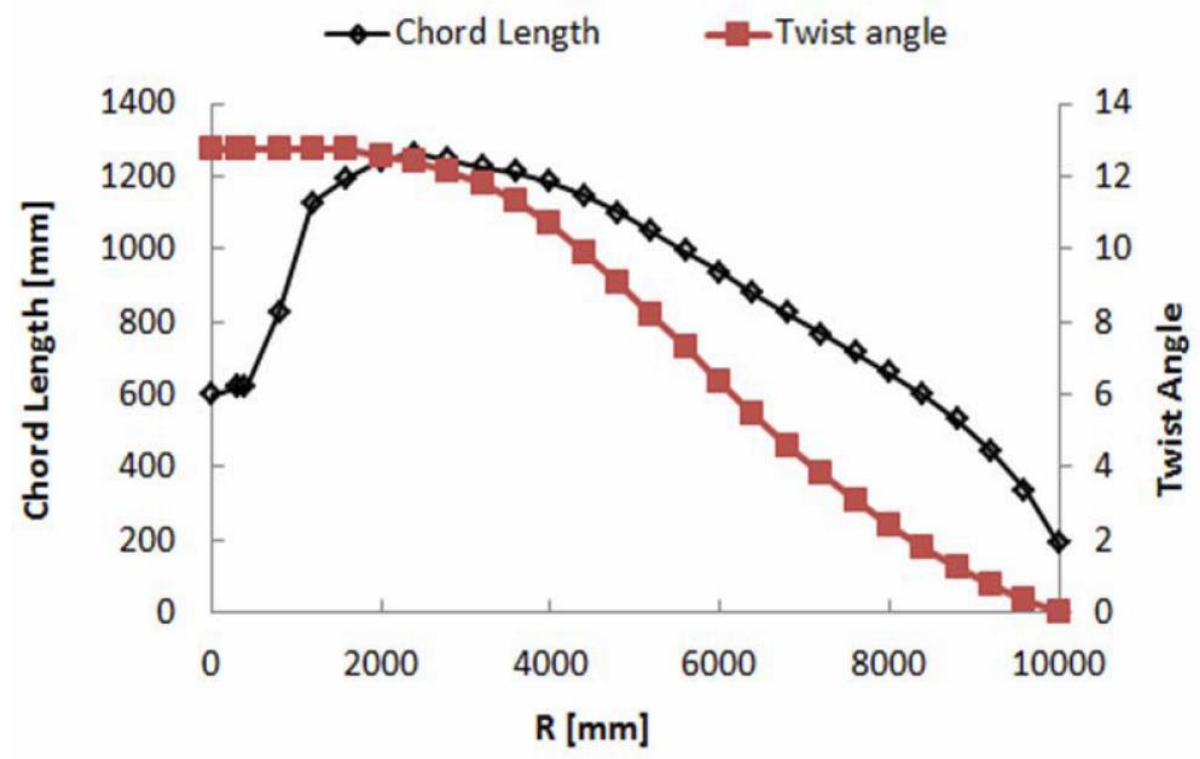

Figura 39. Representación de las distribuciones de ángulos de ataque geométricos y cuerdas [28]

\section{Ángulos de paso geométrico y secciones de pala}

La distribución de ángulos de paso geométrico se ha obtenido en base a la Figura 39, como se ha comentado anteriormente, aunque se considera, al igual que se hizo en el aparatado anterior, que éstos están medidos con una referencia vertical, de ahí que en la distribución el ángulo de paso en el extremo de pala sea cero, es decir, $90^{\circ}$ con la referencia horizontal.

Por lo tanto, para adaptar los valores de esta distribución al procedimiento de cálculo, en el que se utiliza una referencia horizontal, como se puede ver en la Figura 18 , se restará a $90^{\circ}$ la cantidad correspondiente de cada sección.

La pala se divide en 20 secciones de igual longitud $\delta r_{k}$, y los perfiles en cada sección de pala están definidos por el DU91-W2-250. Las curvas de coeficientes de este perfil se han encontrado en una publicación dedicada a las turbinas eólicas [59]. 


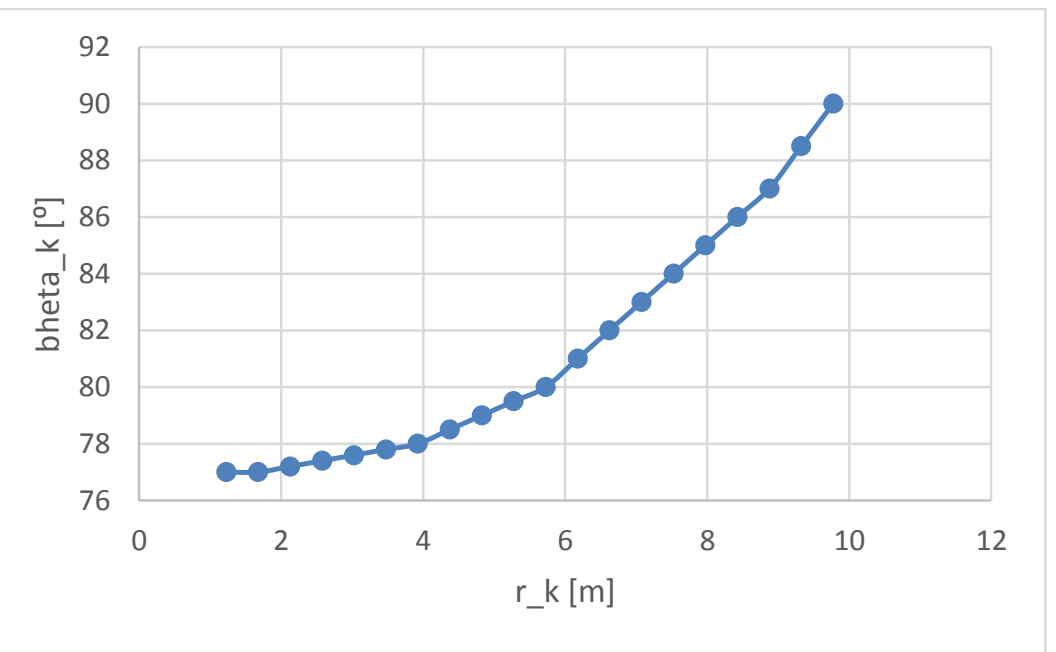

Figura 40. Representación de la distribución de ángulos de paso geométrico $\boldsymbol{\beta}_{k}$

\begin{tabular}{|c|c|c|c|c|c|}
\hline SECTION no & $\boldsymbol{r}_{\boldsymbol{k}}[\boldsymbol{m}]$ & $\boldsymbol{\delta}_{\boldsymbol{k}}[\boldsymbol{m}]$ & $\left.\boldsymbol{\beta}_{\boldsymbol{k}}{ }{ }^{\circ}\right]$ & $\boldsymbol{c}_{\boldsymbol{k}}[\boldsymbol{m}]$ & perfil \\
\hline 1 & 1,225 & 0,45 & 77 & 0,600 & DU91-W2-250 \\
\hline 2 & 1,675 & 0,45 & 77 & 0,800 & DU91-W2-250 \\
\hline 3 & 2,125 & 0,45 & 77,2 & 1,200 & DU91-W2-250 \\
\hline 4 & 2,575 & 0,45 & 77,4 & 1,250 & DU91-W2-250 \\
\hline 5 & 3,025 & 0,45 & 77,6 & 1,200 & DU91-W2-250 \\
\hline 6 & 3,475 & 0,45 & 77,8 & 1,150 & DU91-W2-250 \\
\hline 7 & 3,925 & 0,45 & 78 & 1,100 & DU91-W2-250 \\
\hline 8 & 4,375 & 0,45 & 78,5 & 1,050 & DU91-W2-250 \\
\hline 9 & 4,825 & 0,45 & 79 & 1,000 & DU91-W2-250 \\
\hline 10 & 5,275 & 0,45 & 79,5 & 0,950 & DU91-W2-250 \\
\hline 11 & 5,725 & 0,45 & 80 & 0,900 & DU91-W2-250 \\
\hline 12 & 6,175 & 0,45 & 81 & 0,850 & DU91-W2-250 \\
\hline 13 & 6,625 & 0,45 & 82 & 0,800 & DU91-W2-250 \\
\hline 14 & 7,075 & 0,45 & 83 & 0,750 & DU91-W2-250 \\
\hline 15 & 7,525 & 0,45 & 84 & 0,700 & DU91-W2-250 \\
\hline 16 & 7,975 & 0,45 & 85 & 0,625 & DU91-W2-250 \\
\hline 17 & 8,425 & 0,45 & 86 & 0,550 & DU91-W2-250 \\
\hline 18 & 8,875 & 0,45 & 87 & 0,450 & DU91-W2-250 \\
\hline 19 & 9,325 & 0,45 & 88,5 & 0,350 & DU91-W2-250 \\
\hline 20 & 9,775 & 0,45 & 90 & 0,200 & DU91-W2-250 \\
\hline
\end{tabular}

Tabla 24. Definición de la geometría de pala 


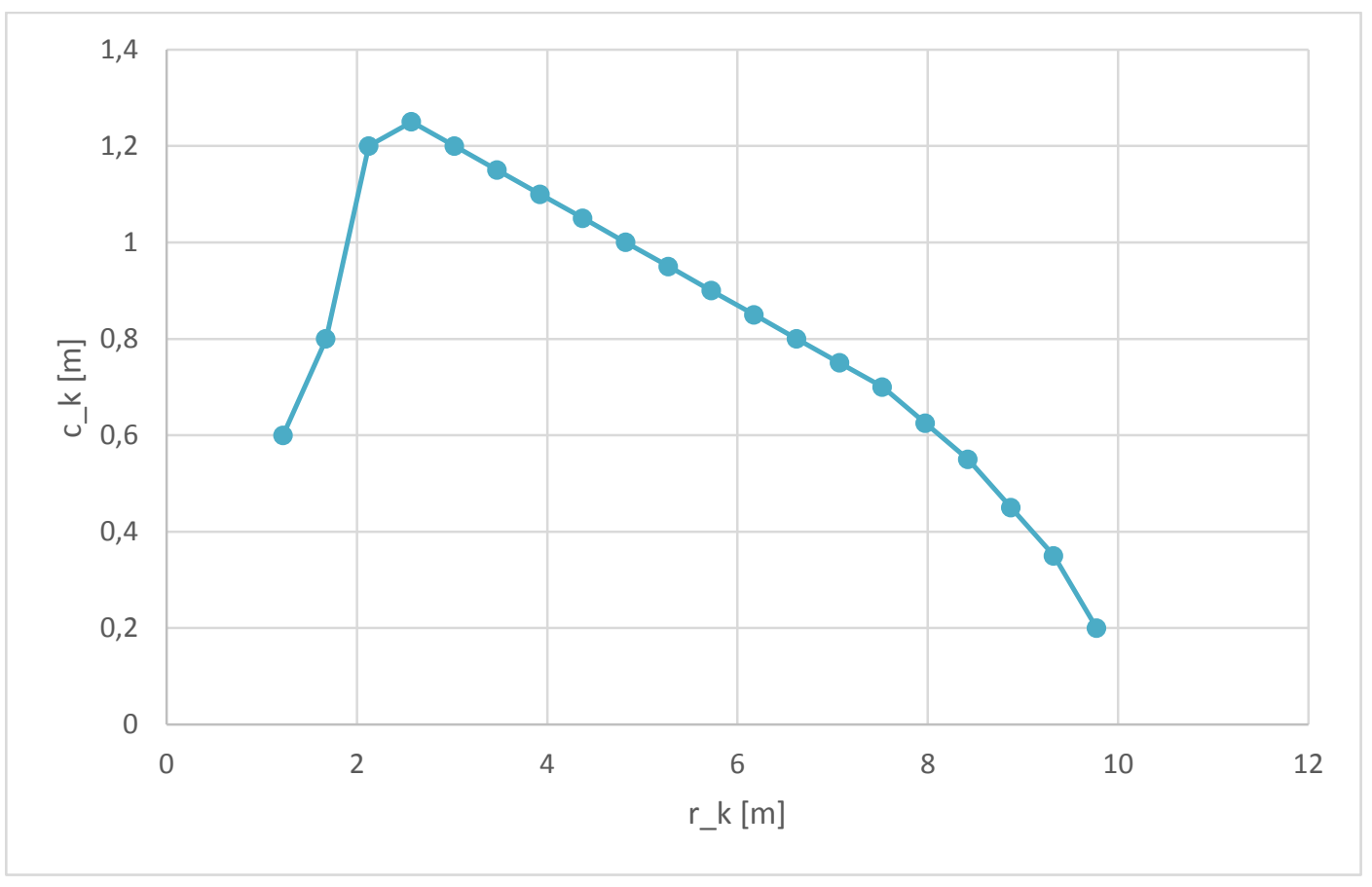

Figura 41. Representación de la distribución de cuerdas $c_{k}$

\section{Cálculo de las variables auxiliares}

De acuerdo a los pasos establecidos para la búsqueda de soluciones de las ecuaciones propuestas en este procedimiento de cálculo, y como se vio en el apartado anterior, el primer paso a realizar es obtener el valor de las variables auxiliares $\varphi_{k}, \lambda\left(r_{k}\right), \mu\left(r_{k}\right)$.

En el caso de $\varphi_{k}$, se calcula directamente, ya que su expresiones son relativamente sencillas, mientras que para $\lambda\left(r_{k}\right)$ y $\mu\left(r_{k}\right)$ hay que hacer un paso intermedio ya que se trata de funciones integrales y requieren del uso de una herramienta adicional que permita resolver expresiones de este tipo. Al igual que en el apartado anterior, para obtenerlos, se ha utilizado el fichero Cálculo de mu y lambda.mw.

\begin{tabular}{|c|c|c|c|c|}
\hline SECTION no & $\boldsymbol{r}_{\boldsymbol{k}}[\boldsymbol{m}]$ & $\boldsymbol{\varphi}_{\boldsymbol{k}}$ & $\boldsymbol{\mu}\left(\boldsymbol{r}_{\boldsymbol{k}}\right)$ & $\boldsymbol{\lambda}\left(\boldsymbol{r}_{\boldsymbol{k}}\right)$ \\
\hline 1 & 1,225 & 1,47793 & 5,09 & 7,5992 \\
\hline 2 & 1,675 & 1,12337 & 0,78302 & 2,6613 \\
\hline 3 & 2,125 & 0,88180 & 0,21016 & 1,5219 \\
\hline 4 & 2,575 & 0,72688 & 0,07124 & 1,017 \\
\hline 5 & 3,025 & 0,62628 & 0,027954 & 0,75136 \\
\hline 6 & 3,475 & 0,55940 & 0,011912 & 0,59847 \\
\hline 7 & 3,925 & 0,51450 & 0,005154 & 0,50593 \\
\hline 8 & 4,375 & 0,48492 & 0,00208 & 0,44931 \\
\hline 9 & 4,825 & 0,46689 & 0,00053 & 0,41393 \\
\hline 10 & 5,275 & 0,45833 & 0,00011 & 0,40133 \\
\hline 11 & 5,725 & 0,45833 & 0,00011 & 0,40133 \\
\hline 12 & 6,175 & 0,46689 & 0,00053 & 0,41393 \\
\hline
\end{tabular}




\begin{tabular}{|c|c|c|c|c|}
\hline SECTION no & $\boldsymbol{r}_{\boldsymbol{k}}[\boldsymbol{m}]$ & $\boldsymbol{\varphi}_{\boldsymbol{k}}$ & $\boldsymbol{\mu}\left(\boldsymbol{r}_{\boldsymbol{k}}\right)$ & $\boldsymbol{\lambda}\left(\boldsymbol{r}_{\boldsymbol{k}}\right)$ \\
\hline 13 & 6,625 & 0,48492 & 0,00208 & 0,44931 \\
\hline 14 & 7,075 & 0,51450 & 0,005154 & 0,50593 \\
\hline 15 & 7,525 & 0,55940 & 0,011912 & 0,59847 \\
\hline 16 & 7,975 & 0,62628 & 0,027954 & 0,75136 \\
\hline 17 & 8,425 & 0,72688 & 0,07124 & 1,017 \\
\hline 18 & 8,875 & 0,88180 & 0,21016 & 1,5219 \\
\hline 19 & 9,325 & 1,12337 & 0,78302 & 2,6613 \\
\hline 20 & 9,775 & 1,47793 & 5,09 & 7,5992 \\
\hline
\end{tabular}

Tabla 25. Valores de las variables auxiliares $\varphi_{k}, \lambda\left(r_{k}\right)$ y $\mu\left(r_{k}\right)$

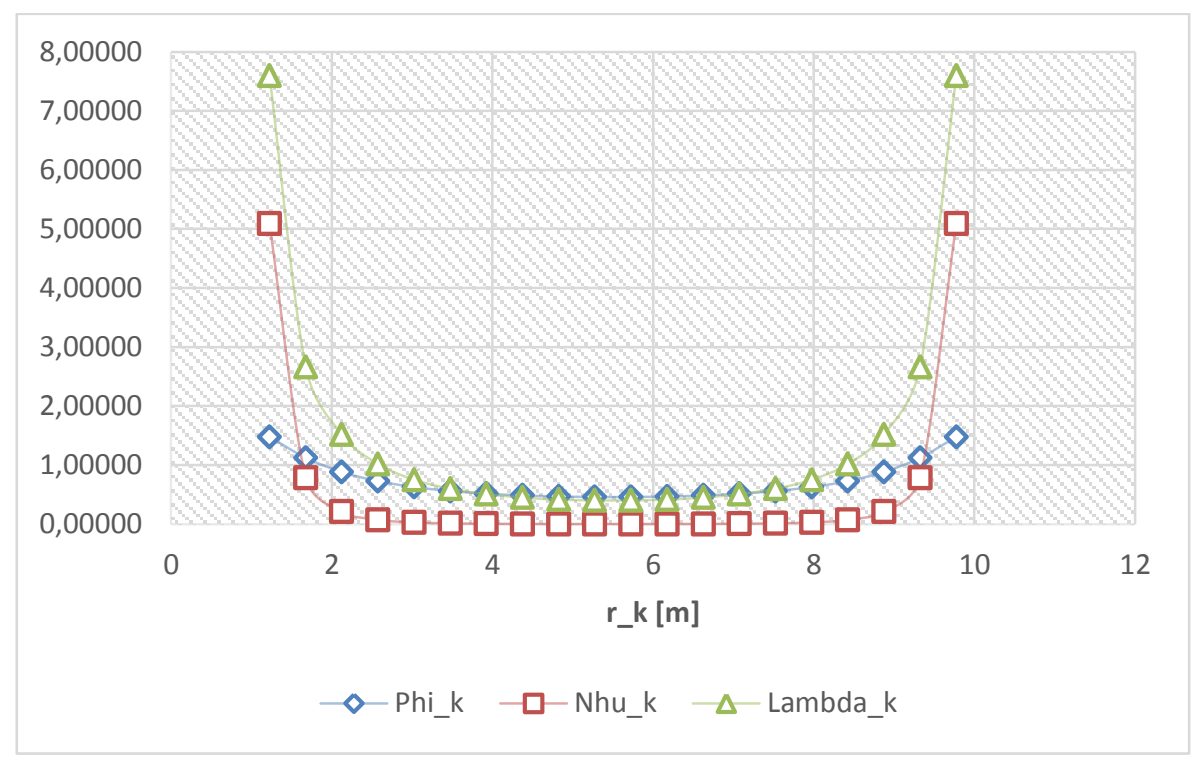

Figura 42. Representación gráfica de $\varphi_{k}, \lambda\left(r_{k}\right)$ y $\mu\left(r_{k}\right)$ con respecto al radio de cada sección de pala

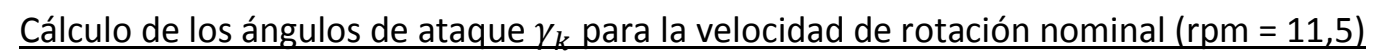

Con todas las consideraciones anteriores se procede a ejecutar la rutina de cálculo que implementa el procedimiento propuesto en esta tesis.

Esta geometría inicial de pala se ha mostrado incompatible con las ecuaciones del procedimiento alternativo, es decir, no se ha podido calcular una distribución de ángulos de ataque $\gamma_{k}$ que satisfaga la condición establecida de que la suma de las variables auxiliares $\psi_{k}$ sea próxima a cero.

Sin embargo, con la implementación del método basado en la Teoría de Elementos de pala se obtiene un coeficiente de potencia de 0,421 y una potencia de rotor de $939 \mathrm{~kW}$.

\begin{tabular}{|c|c|c|c|c|c|c|c|c|}
\hline $\mathbf{n} \mathbf{0}$ & $\mathbf{r}[\mathbf{k}][\mathrm{m}]$ & delta[k][m] & bheta[k][0] & $\mathbf{c [ k ] [ m ]}$ & PERFIL & cl[k] & cd[k] & gamma[k][0] \\
\hline 1 & 1,225 & 0,45 & 77 & 0,6 & DU91-W2-250 & 0,515 & 0,703 & 45,42 \\
\hline
\end{tabular}




\begin{tabular}{|c|c|c|c|c|c|c|c|c|}
\hline $\mathbf{n} \mathbf{0}$ & $\mathbf{r}[\mathbf{k}][\mathbf{m}]$ & delta[k][m] & bheta[k][0] & $\mathbf{c}[\mathbf{k}][\mathbf{m}]$ & PERFIL & cl[k] & cd[k] & gamma[k][0] \\
\hline 2 & 1,675 & 0,45 & 77 & 0,8 & DU91-W2-250 & 0,498 & 0,483 & 36,95 \\
\hline 3 & 2,125 & 0,45 & 77,2 & 1,2 & DU91-W2-250 & 0,529 & 0,332 & 30,36 \\
\hline 4 & 2,575 & 0,45 & 77,4 & 1,25 & DU91-W2-250 & 0,598 & 0,239 & 25,13 \\
\hline 5 & 3,025 & 0,45 & 77,59 & 1,2 & DU91-W2-250 & 0,692 & 0,175 & 20,97 \\
\hline 6 & 3,475 & 0,45 & 77,79 & 1,15 & DU91-W2-250 & 1,153 & 0,085 & 14,32 \\
\hline 7 & 3,924 & 0,45 & 78 & 1,1 & DU91-W2-250 & 1,386 & 0,027 & 10,78 \\
\hline 8 & 4,375 & 0,45 & 78,5 & 1,05 & DU91-W2-250 & 1,427 & 0,014 & 8,93 \\
\hline 9 & 4,825 & 0,45 & 79 & 1 & DU91-W2-250 & 1,274 & 0,013 & 7,33 \\
\hline 10 & 5,275 & 0,45 & 79,5 & 0,95 & DU91-W2-250 & 1,146 & 0,012 & 6,18 \\
\hline 11 & 5,724 & 0,45 & 80 & 0,9 & DU91-W2-250 & 1,057 & 0,012 & 5,39 \\
\hline 12 & 6,174 & 0,45 & 81 & 0,85 & DU91-W2-250 & 1,052 & 0,012 & 5,36 \\
\hline 13 & 6,625 & 0,45 & 82 & 0,8 & DU91-W2-250 & 1,065 & 0,012 & 5,46 \\
\hline 14 & 7,075 & 0,45 & 83 & 0,75 & DU91-W2-250 & 1,089 & 0,012 & 5,67 \\
\hline 15 & 7,525 & 0,45 & 84 & 0,7 & DU91-W2-250 & 1,122 & 0,012 & 5,97 \\
\hline 16 & 7,974 & 0,45 & 85 & 0,625 & DU91-W2-250 & 1,176 & 0,012 & 6,43 \\
\hline 17 & 8,425 & 0,45 & 86 & 0,55 & DU91-W2-250 & 1,238 & 0,012 & 6,99 \\
\hline 18 & 8,875 & 0,45 & 87 & 0,45 & DU91-W2-250 & 1,315 & 0,013 & 7,72 \\
\hline 19 & 9,324 & 0,45 & 88,5 & 0,35 & DU91-W2-250 & 1,432 & 0,014 & 9,05 \\
\hline 20 & 9,775 & 0,45 & 90 & 0,2 & DU91-W2-250 & 1,335 & 0,037 & 11,52 \\
\hline
\end{tabular}

Tabla 26. Distribución de ángulos de ataque $\gamma_{\mathrm{k}}$ y coeficientes de sustentación y arrastre obtenidas con la implementación del método de elementos de pala para $\mathrm{rpm}=11,5$

\section{$\underline{\text { Rotación de } 16^{\circ} \text { de la geometría de pala }}$}

Un factor que es crítico para el coeficiente de potencia del rotor es la distribución de ángulos de paso geométrico de las secciones de pala. En la geometría de partida propuesta y representada en la Tabla 24 se puede ver que la variación del ángulo entre las secciones de núcleo del rotor y del extremo de pala es de unos $13^{\circ}$. En esta distribución de ángulos inicial de ángulos de paso se ha considerado que el ángulo de paso del extremo de pala es igual a $90^{\circ}$, como se comentó anteriormente.

Sin embargo, si se resta $16^{\circ}$ al extremo de pala, éste alcanzará el valor de $74^{\circ}$, y análogamente se resta también $16^{\circ}$ al ángulo de paso geométrico de todas las secciones de pala se obtiene una geometría de pala igual a la inicial, sólo que constructivamente se girará $16^{\circ}$.

Esta pequeña transformación, resulta crítica para la búsqueda de soluciones compatibles del ángulo de ataque $\gamma_{\mathrm{k}} \mathrm{y}$ para el coeficiente de potencia del rotor, para el que, con esta pequeña transformación, se ha obtenido un valor de 0,458 y una potencia del rotor de 1020 kW.

La suma total de las variables $\psi_{k}$ es de $0,042 \mathrm{~m}^{4} / \mathrm{s}^{2}$ y la circulación $\Gamma$ es $8,315 \mathrm{~m}^{2} / \mathrm{s}$.

En la siguiente tabla se muestran las distribuciones de la nueva geometría obtenida y la distribución de ángulos de ataque $\gamma_{\mathrm{k}}$. 


\begin{tabular}{|c|c|c|c|c|c|c|c|c|}
\hline no & r_k[m] & delta_k[m] & bheta_k[0] & c_k[m] & PERFIL & gamma_k[] & cl_k & cd_k \\
\hline 1 & 1,225 & 0,45 & 61 & 0,6 & DU91-W2-250 & 10,69 & 1,392 & 0,026 \\
\hline 2 & 1,675 & 0,45 & 61 & 0,8 & DU91-W2-250 & 10,05 & 1,423 & 0,02 \\
\hline 3 & 2,125 & 0,45 & 61,2 & 1,2 & DU91-W2-250 & 9,41 & 1,438 & 0,015 \\
\hline 4 & 2,575 & 0,45 & 61,39 & 1,25 & DU91-W2-250 & 8,77 & 1,414 & 0,014 \\
\hline 5 & 3,025 & 0,45 & 61,6 & 1,2 & DU91-W2-250 & 8,13 & 1,356 & 0,013 \\
\hline 6 & 3,475 & 0,45 & 61,79 & 1,15 & DU91-W2-250 & 7,48 & 1,29 & 0,013 \\
\hline 7 & 3,924 & 0,45 & 62 & 1,1 & DU91-W2-250 & 6,84 & 1,221 & 0,012 \\
\hline 8 & 4,375 & 0,45 & 62,5 & 1,05 & DU91-W2-250 & 6,2 & 1,149 & 0,012 \\
\hline 9 & 4,825 & 0,45 & 63 & 1 & DU91-W2-250 & 5,56 & 1,076 & 0,012 \\
\hline 10 & 5,275 & 0,45 & 63,5 & 0,95 & DU91-W2-250 & 4,92 & 1,001 & 0,011 \\
\hline 11 & 5,724 & 0,45 & 64 & 0,9 & DU91-W2-250 & 4,27 & 0,924 & 0,011 \\
\hline 12 & 6,174 & 0,45 & 65 & 0,85 & DU91-W2-250 & 3,63 & 0,846 & 0,011 \\
\hline 13 & 6,625 & 0,45 & 66 & 0,8 & DU91-W2-250 & 2,99 & 0,768 & 0,011 \\
\hline 14 & 7,075 & 0,45 & 67 & 0,75 & DU91-W2-250 & 2,35 & 0,689 & 0,01 \\
\hline 15 & 7,525 & 0,45 & 68 & 0,7 & DU91-W2-250 & 1,71 & 0,61 & 0,01 \\
\hline 16 & 7,974 & 0,45 & 69 & 0,625 & DU91-W2-250 & 1,06 & 0,529 & 0,01 \\
\hline 17 & 8,425 & 0,45 & 70 & 0,55 & DU91-W2-250 & 0,42 & 0,449 & 0,01 \\
\hline 18 & 8,875 & 0,45 & 71 & 0,45 & DU91-W2-250 & $-0,21$ & 0,367 & 0,01 \\
\hline 19 & 9,324 & 0,45 & 72,5 & 0,35 & DU91-W2-250 & $-0,85$ & 0,285 & 0,01 \\
\hline 20 & 9,775 & 0,45 & 74 & 0,2 & DU91-W2-250 & $-1,49$ & 0,203 & 0,01 \\
\hline
\end{tabular}

Tabla 27. Nueva geometría de pala modificada por rotación $16^{\circ}$ de los ángulos de paso geométricos y la distribución de ángulos de ataque $\gamma_{\mathrm{k}}$ para $\mathrm{rpm}=11,5$

La nueva geometría de pala ha dado buenos resultados con el procedimiento alternativo, sin embargo, resulta ser incompatible con la metodología de cálculo basada en los elementos de pala. Se ha obtenido un valor negativo del coeficiente de potencia.

Desde el punto de vista estructural de pueden comparar las cargas a las que está sometida la pala según los elementos de pala para la geometría inicial, y para la modificada restando $16^{\circ}$ a los ángulos de paso geométrico, ya que son funcionamientos del rotor equivalentes.

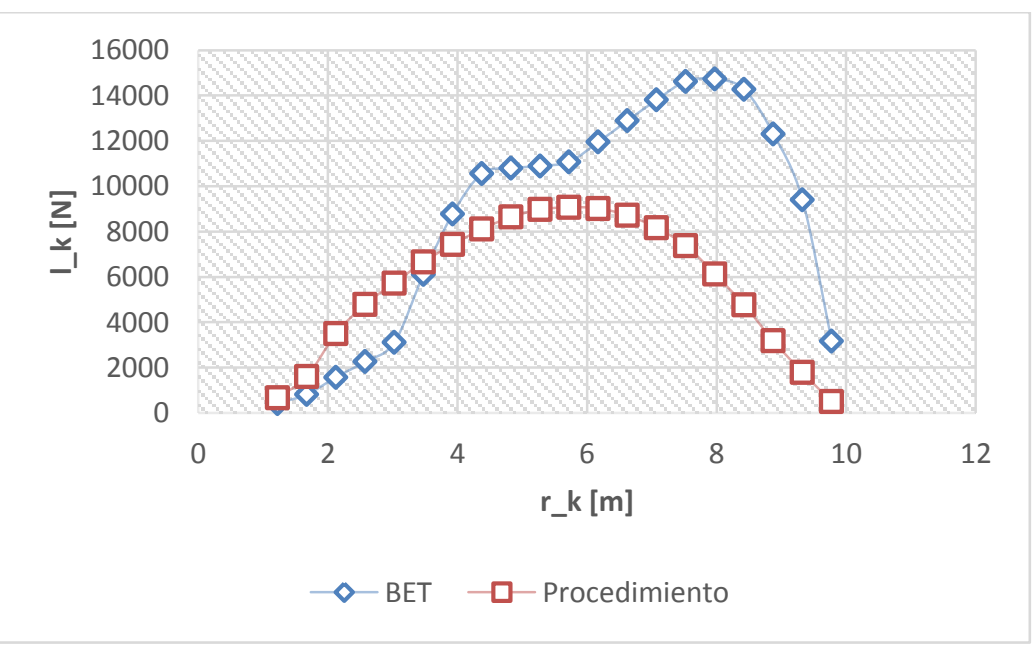

Figura 43. Gráfica comparativa de las fuerzas $l_{k}$ para rpm $=11,5$ y $U=2,4 \mathrm{~m} / \mathrm{s}$ 


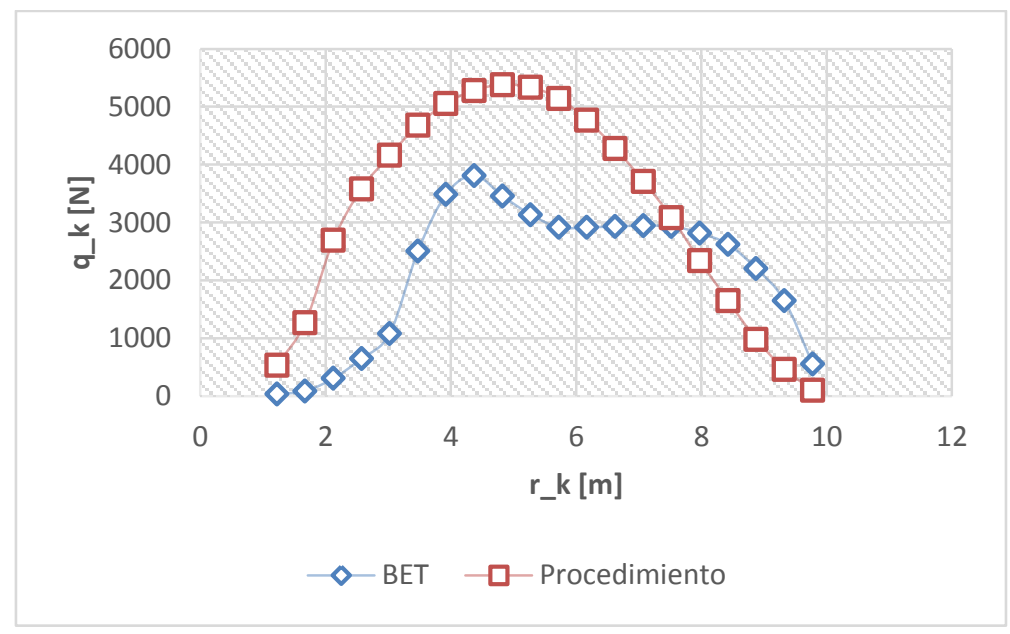

Figura 44. Gráfica comparativa de las fuerzas $q_{k}$ para rpm $=11,5$ y U $=2,4 \mathrm{~m} / \mathrm{s}$

\section{Comportamiento del rotor a distintas TSR}

La velocidad de rotación del rotor es un parámetro que influye directamente en el coeficiente de potencia y en la potencia que entrega el rotor. Cuanto mayor es esta velocidad, en principio, mayor es el coeficiente de potencia como se deduce de la expresión general de coeficiente de potencia en la ecuación Ec. 101.

Sin embargo, a velocidades de rotación demasiado altas se puede producir que el ángulo de ataque en las secciones aumente y se produzca el fenómeno de pérdida haciendo que caiga bruscamente la potencia entrega y por tanto, el coeficiente de potencia.

Para reducir la velocidad de rotación, hay que frenar el eje del rotor hasta que se alcance el nuevo régimen de funcionamiento, esto implica que hay que aumentar el momento resistente al giro del rotor hasta que se iguale éste con el producido por las fuerzas de sustentación en las palas del rotor y que se transmite hasta el eje.

El rotor en estudio de este apartado se ha diseñado para una velocidad de rotación nominal de 11,5 revoluciones por minuto y una velocidad de corriente de $2,4 \mathrm{~m} / \mathrm{s}$, y con ayuda del procedimiento de cálculo de esta tesis se va a estudiar el comportamiento a distintas velocidades de rotación. Se comprobará el coeficiente de potencia y la potencia entregada así como la evolución de la circulación $\Gamma$.

Se va a suponer que la distribución de ángulos de ataque sigue una distribución lineal al igual que para el caso ya estudiado anteriormente en el que la velocidad de rotación es de 11,5 revoluciones por minuto y la velocidad de la corriente es de 2,4 m/s. Esta distribución lineal cumple la condición de que se cumpla la relación $\sum_{\mathrm{k}=1}^{\mathrm{n}} \psi\left(\mathrm{r}_{\mathrm{k}}\right) \delta \mathrm{r}_{\mathrm{k}} \approx 0$.

En primer lugar se estudiará el caso en que rpm $=9(\mathrm{TSR}=3,927)$. Con esta velocidad de rotación se obtiene un coeficiente de potencia de $c_{p}=0,28$ y una potencia entregada en el rotor $\mathrm{P}=626 \mathrm{~kW}$; la circulación de los vórtices es $\Gamma=7,711 \mathrm{~m}^{2} / \mathrm{s}$. 
Estos resultados se han obtenido con una distribución de ángulos de ataque que se muestra en la siguiente Tabla 28 , y por otro lado, el valor del sumatorio de $\psi\left(r_{k}\right)$ es igual a $0,028 \mathrm{~m}^{4} / \mathrm{s}^{2}$.

\begin{tabular}{|c|c|c|c|c|c|c|c|c|}
\hline no & r_k[m] & delta_k[m] & bheta_k[0] & c_k[m] & PERFIL & gamma_k[-] & cl_k & cd_k \\
\hline 1 & 1,225 & 0,45 & 61 & 0,6 & DU91-W2-250 & 2,9 & 0,756 & 0,011 \\
\hline 2 & 1,675 & 0,45 & 61 & 0,8 & DU91-W2-250 & 3,01 & 0,77 & 0,011 \\
\hline 3 & 2,125 & 0,45 & 61,2 & 1,2 & DU91-W2-250 & 3,12 & 0,783 & 0,011 \\
\hline 4 & 2,575 & 0,45 & 61,39 & 1,25 & DU91-W2-250 & 3,23 & 0,797 & 0,011 \\
\hline 5 & 3,025 & 0,45 & 61,6 & 1,2 & DU91-W2-250 & 3,34 & 0,811 & 0,011 \\
\hline 6 & 3,475 & 0,45 & 61,79 & 1,15 & DU91-W2-250 & 3,45 & 0,824 & 0,011 \\
\hline 7 & 3,924 & 0,45 & 62 & 1,1 & DU91-W2-250 & 3,56 & 0,838 & 0,011 \\
\hline 8 & 4,375 & 0,45 & 62,5 & 1,05 & DU91-W2-250 & 3,67 & 0,851 & 0,011 \\
\hline 9 & 4,825 & 0,45 & 63 & 1 & DU91-W2-250 & 3,78 & 0,864 & 0,011 \\
\hline 10 & 5,275 & 0,45 & 63,5 & 0,95 & DU91-W2-250 & 3,89 & 0,878 & 0,011 \\
\hline 11 & 5,724 & 0,45 & 64 & 0,9 & DU91-W2-250 & 4 & 0,891 & 0,011 \\
\hline 12 & 6,174 & 0,45 & 65 & 0,85 & DU91-W2-250 & 4,11 & 0,904 & 0,011 \\
\hline 13 & 6,625 & 0,45 & 66 & 0,8 & DU91-W2-250 & 4,22 & 0,917 & 0,011 \\
\hline 14 & 7,075 & 0,45 & 67 & 0,75 & DU91-W2-250 & 4,33 & 0,93 & 0,011 \\
\hline 15 & 7,525 & 0,45 & 68 & 0,7 & DU91-W2-250 & 4,44 & 0,944 & 0,011 \\
\hline 16 & 7,974 & 0,45 & 69 & 0,625 & DU91-W2-250 & 4,55 & 0,957 & 0,011 \\
\hline 17 & 8,425 & 0,45 & 70 & 0,55 & DU91-W2-250 & 4,66 & 0,97 & 0,011 \\
\hline 18 & 8,875 & 0,45 & 71 & 0,45 & DU91-W2-250 & 4,77 & 0,983 & 0,011 \\
\hline 19 & 9,324 & 0,45 & 72,5 & 0,35 & DU91-W2-250 & 4,88 & 0,997 & 0,011 \\
\hline 20 & 9,775 & 0,45 & 74 & 0,2 & DU91-W2-250 & 5 & 1,01 & 0,012 \\
\hline
\end{tabular}

Tabla 28. Nueva distribución de los ángulos de ataque $\gamma_{k}$ para $r p m=9$ y $U=2,4 \mathrm{~m} / \mathrm{s}$

Esta velocidad de rotación se introduce también el método de elementos de pala, para la geometría de pala definida inicialmente en la Tabla 24, para establecer criterios comparativos entre los resultados con ambos métodos de cálculo.

Puesto que, como se vio anteriormente, la nueva geometría de pala definida con una disminución de $16^{\circ}$ de los ángulos de paso geométrico, resultó tener una condición de funcionamiento equivalente con respecto al coeficiente de potencia, y por ello es de interés comparar ambas geometrías con las dos metodologías implementadas.

Así pues, con el método de elementos de pala el coeficiente de potencia obtenido es igual a 0,263 y la potencia del rotor es de $587 \mathrm{~kW}$.

Los ángulos de ataque, coeficientes de sustentación y arrastre se muestran en la siguiente Tabla 29.

\begin{tabular}{|c|c|c|c|c|c|c|c|c|}
\hline $\mathbf{n o}$ & $\mathbf{r}[\mathbf{k}][\mathbf{m}]$ & delta[k][m] & bheta[k][0] & $\mathbf{c}[\mathbf{k}][\mathbf{m}]$ & PERFIL & gamma[k][o] & $\mathbf{c l [ k ]}$ & $\mathbf{c d}[\mathbf{k}]$ \\
\hline 1 & 1,225 & 0,45 & 77 & 0,6 & DU91-W2-250 & 51,3 & 0,54 & 0,843 \\
\hline 2 & 1,675 & 0,45 & 77 & 0,8 & DU91-W2-250 & 43,66 & 0,499 & 0,633 \\
\hline 3 & 2,125 & 0,45 & 77,2 & 1,2 & DU91-W2-250 & 37,35 & 0,491 & 0,471 \\
\hline 4 & 2,575 & 0,45 & 77,4 & 1,25 & DU91-W2-250 & 32,08 & 0,515 & 0,364 \\
\hline
\end{tabular}




\begin{tabular}{|c|c|c|c|c|c|c|c|c|}
\hline no & r[k][m] & delta[k][m] & bheta[k][] & c[k][m] & PERFIL & gamma[k][] & cl[k] & cd[k] \\
\hline 5 & 3,025 & 0,45 & 77,59 & 1,2 & DU91-W2-250 & 27,69 & 0,559 & 0,284 \\
\hline 6 & 3,475 & 0,45 & 77,79 & 1,15 & DU91-W2-250 & 24,03 & 0,618 & 0,222 \\
\hline 7 & 3,924 & 0,45 & 78 & 1,1 & DU91-W2-250 & 20,97 & 0,691 & 0,176 \\
\hline 8 & 4,375 & 0,45 & 78,5 & 1,05 & DU91-W2-250 & 18,7 & 0,764 & 0,144 \\
\hline 9 & 4,825 & 0,45 & 79 & 1 & DU91-W2-250 & 13,73 & 1,173 & 0,076 \\
\hline 10 & 5,275 & 0,45 & 79,5 & 0,95 & DU91-W2-250 & 12,38 & 1,261 & 0,053 \\
\hline 11 & 5,724 & 0,45 & 80 & 0,9 & DU91-W2-250 & 11,23 & 1,356 & 0,033 \\
\hline 12 & 6,174 & 0,45 & 81 & 0,85 & DU91-W2-250 & 10,8 & 1,385 & 0,027 \\
\hline 13 & 6,625 & 0,45 & 82 & 0,8 & DU91-W2-250 & 10,55 & 1,4 & 0,024 \\
\hline 14 & 7,075 & 0,45 & 83 & 0,75 & DU91-W2-250 & 10,46 & 1,405 & 0,023 \\
\hline 15 & 7,525 & 0,45 & 84 & 0,7 & DU91-W2-250 & 10,51 & 1,402 & 0,024 \\
\hline 16 & 7,974 & 0,45 & 85 & 0,625 & DU91-W2-250 & 10,76 & 1,388 & 0,027 \\
\hline 17 & 8,425 & 0,45 & 86 & 0,55 & DU91-W2-250 & 11,11 & 1,365 & 0,031 \\
\hline 18 & 8,875 & 0,45 & 87 & 0,45 & DU91-W2-250 & 13 & 1,212 & 0,064 \\
\hline 19 & 9,324 & 0,45 & 88,5 & 0,35 & DU91-W2-250 & 13,77 & 1,171 & 0,076 \\
\hline 20 & 9,775 & 0,45 & 90 & 0,2 & DU91-W2-250 & 14,6 & 1,146 & 0,089 \\
\hline
\end{tabular}

Tabla 29. Nueva distribución de los ángulos de ataque $\gamma_{k}$ para rpm $=9$ y $U=2,4 \mathrm{~m} / \mathrm{s}$ (BET)

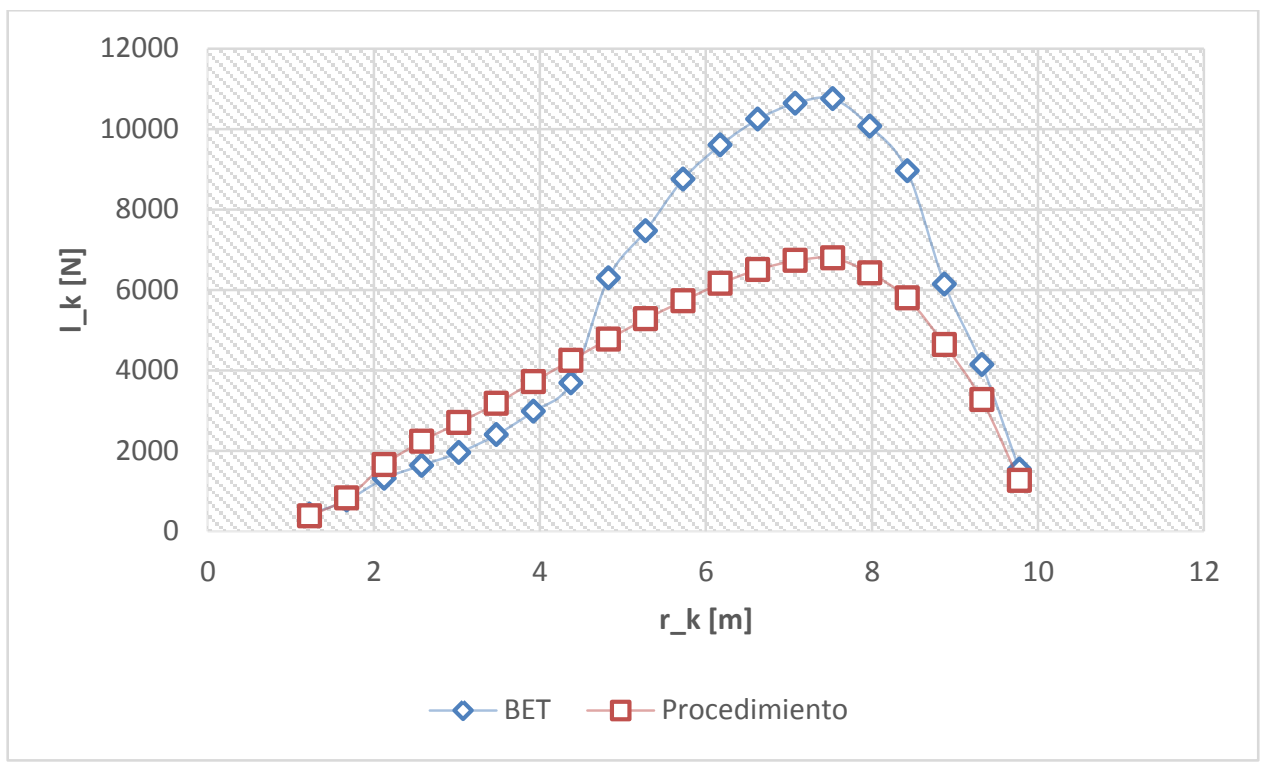

Figura 45. Gráfica comparativa de las fuerzas $l_{k}$ para $\mathrm{rpm}=9$ y $\mathrm{U}=2,4 \mathrm{~m} / \mathrm{s}$

Con una velocidad de rotor de $12,5 \mathrm{rpm}$ y $\mathrm{U}=2,4 \mathrm{~m} / \mathrm{s}(\mathrm{TSR}=5,454)$, se vuelven a recalcular las distribuciones de ángulos de ataque con el procedimiento y con el método de elementos de pala.

Por un lado, el coeficiente de potencia obtenido con el procedimiento alcanza un valor igual 0,575 y 0,441 con el método de elementos de pala. 


\section{Estudio de la torsión de las secciones de pala}

El estudio de la torsión en las secciones de pala se realizará suponiendo que la pala ha sido construida con un refuerzo macizo principal, de sección circular, cuadrada o rectangular. Con esta disposición, se calculará cómo quedan afectados los ángulos de ataque de la pala y de este modo se puede obtener como afecta la torsión de las palas al rendimiento del rotor y a la potencia del rotor.

\section{Refuerzo principal macizo de sección circular de 0,15 m de diámetro}

En primer lugar se supone un refuerzo macizo de acero de sección circular y de 0,15 metros de diámetro. Con esto se obtiene el coeficiente $\mathrm{K}$ de la ecuación Ec. 128, que de acuerdo a la fórmula de la Tabla 3 queda,

$$
\mathrm{K}=\frac{1}{2} \pi\left(\frac{0,15}{2}\right)^{4}=4,97 \cdot 10^{-5} \mathrm{~m}^{4}
$$

En la Tabla 30, se muestra como queda afectada la distribución de ángulos de paso geométricos de las secciones de pala.

\begin{tabular}{|c|c|c|c|c|c|c|}
\hline SECTION no & $\mathbf{r}$-k[m] & delta_k[m] & bheta_k[0] & $\sum_{i=1}^{n} \boldsymbol{Q}_{\boldsymbol{k}}[\mathbf{N m}]$ & $\boldsymbol{\theta}_{\boldsymbol{k}}\left[^{\circ}\right]$ & $\left.\boldsymbol{\beta}^{\prime}{ }_{\boldsymbol{k}}{ }^{\circ}\right]$ \\
\hline 1 & 1,225 & 0,45 & 61 & 219,688 & 0,004 & 61,00 \\
\hline 2 & 1,675 & 0,45 & 61 & 582,440 & 0,015 & 61,01 \\
\hline 3 & 2,125 & 0,45 & 61,2 & 1263,829 & 0,040 & 61,24 \\
\hline 4 & 2,575 & 0,45 & 61,4 & 2150,453 & 0,083 & 61,48 \\
\hline 5 & 3,025 & 0,45 & 61,6 & 3203,891 & 0,145 & 61,75 \\
\hline 6 & 3,475 & 0,45 & 61,8 & 4438,441 & 0,231 & 62,03 \\
\hline 7 & 3,925 & 0,45 & 62 & 5864,338 & 0,345 & 62,34 \\
\hline 8 & 4,375 & 0,45 & 62,5 & 7487,753 & 0,490 & 62,99 \\
\hline 9 & 4,825 & 0,45 & 63 & 9310,791 & 0,673 & 63,67 \\
\hline 10 & 5,275 & 0,45 & 63,5 & 11331,498 & 0,895 & 64,39 \\
\hline 11 & 5,725 & 0,45 & 64 & 13543,852 & 1,161 & 65,16 \\
\hline 12 & 6,175 & 0,45 & 65 & 15937,770 & 1,473 & 66,47 \\
\hline 13 & 6,625 & 0,45 & 66 & 18499,104 & 1,835 & 67,83 \\
\hline 14 & 7,075 & 0,45 & 67 & 21209,644 & 2,247 & 69,25 \\
\hline 15 & 7,525 & 0,45 & 68 & 24047,114 & 2,709 & 70,71 \\
\hline 16 & 7,975 & 0,45 & 69 & 26872,173 & 3,209 & 72,21 \\
\hline 17 & 8,425 & 0,45 & 70 & 29629,737 & 3,737 & 73,74 \\
\hline 18 & 8,875 & 0,45 & 71 & 32120,262 & 4,268 & 75,27 \\
\hline 19 & 9,325 & 0,45 & 72,5 & 34249,081 & 4,782 & 77,28 \\
\hline 20 & 9,775 & 0,45 & 74 & 35580,536 & 5,207 & 79,21 \\
\hline & & & & & & \\
\hline
\end{tabular}

Tabla 30. Nueva distribución de los ángulos de ataque teniendo en cuenta el efecto de la torsión en las secciones de pala para $\mathrm{rpm}=11,5$ y considerando un refuerzo principal de sección circular 
Aplicando de nuevo el procedimiento de cálculo teniendo en cuenta la nueva distribución de ángulos de ataque, se obtiene que el coeficiente de potencia disminuye hasta 0,355 y la potencia del rotor que se queda en $791 \mathrm{~kW}$.

\section{$\underline{\text { Refuerzo principal macizo de sección cuadrada y lado de } 0,15 \mathrm{~m}}$}

Para este refuerzo, el coeficiente de proporcionalidad es,

$$
\mathrm{K}=2\left(\frac{0,15}{2}\right)^{4}=7,1191 \cdot 10^{-5} \mathrm{~m}^{4}
$$

Valor que es mayor que el obtenido para el caso anterior del refuerzo de sección circular, esto disminuirá la torsión de las secciones de pala y por tanto el coeficiente de potencia no se verá tan afectado. El coeficiente de potencia obtenido es de 0,373 y la potencia del rotor es de $830 \mathrm{~kW}$.

\begin{tabular}{|c|c|c|c|c|}
\hline SECTION no & $r_{-} \mathbf{k}[\mathrm{m}]$ & $\sum^{n} Q_{k}[N m]$ & $\boldsymbol{\theta}_{\boldsymbol{k}}\left[^{\circ}\right]$ & $\left.\boldsymbol{\beta}_{\boldsymbol{k}}^{\prime}{ }^{\circ}\right]$ \\
\hline 1 & 1,225 & 219,688 & 0,003 & 61,00 \\
\hline 2 & 1,675 & 582,440 & 0,010 & 61,01 \\
\hline 3 & 2,125 & 1263,829 & 0,028 & 61,23 \\
\hline 4 & 2,575 & 2150,453 & 0,058 & 61,46 \\
\hline 5 & 3,025 & 3203,891 & 0,101 & 61,70 \\
\hline 6 & 3,475 & 4438,441 & 0,161 & 61,96 \\
\hline 7 & 3,925 & 5864,338 & 0,241 & 62,24 \\
\hline 8 & 4,375 & 7487,753 & 0,342 & 62,84 \\
\hline 9 & 4,825 & 9310,791 & 0,470 & 63,47 \\
\hline 10 & 5,275 & 11331,498 & 0,625 & 64,12 \\
\hline 11 & 5,725 & 13543,852 & 0,810 & 64,81 \\
\hline 12 & 6,175 & 15937,770 & 1,029 & 66,03 \\
\hline 13 & 6,625 & 18499,104 & 1,281 & 67,28 \\
\hline 14 & 7,075 & 21209,644 & 1,568 & 68,57 \\
\hline 15 & 7,525 & 24047,114 & 1,891 & 69,89 \\
\hline 16 & 7,975 & 26872,173 & 2,240 & 71,24 \\
\hline 17 & 8,425 & 29629,737 & 2,609 & 72,61 \\
\hline 18 & 8,875 & 32120,262 & 2,980 & 73,98 \\
\hline 19 & 9,325 & 34249,081 & 3,338 & 75,84 \\
\hline 20 & 9,775 & 35580,536 & 3,635 & 77,64 \\
\hline
\end{tabular}

Tabla 31. Nueva distribución de los ángulos de ataque teniendo en cuenta el efecto de la torsión en las secciones de pala para $\mathrm{rpm}=11,5$ y considerando un refuerzo principal de sección cuadrada

Comparando el comportamiento de estos refuerzos estudiados, en el caso del refuerzo principal de sección circular, la torsión en la sección de extremo de pala es de $5,2^{\circ}$ y para el de sección cuadrada es de $3,6^{\circ}$. 
Es decir, con un cambio de la geometría de la sección del refuerzo principal se consigue disminuir el efecto de la torsión en las secciones de pala.

Desde el punto de vista constructivo, si se dispone un refuerzo principal circular de diámetro 0,15 m y un refuerzo de sección cuadrada de lado $0,15 \mathrm{~m}$, en las secciones de extremo de pala no se podría alojar un refuerzo de esta naturaleza debido a que las secciones no tienen el suficiente espesor como para poder colocar el refuerzo sin que este sobresalga.

Por tanto, la solución podría ser que el extremo de pala sea macizo, algo que al ser las secciones muy finas no conllevaría utilizar una gran cantidad de material y adicionalmente, el efecto de la torsión en esta zona de la pala se mitigaría con lo que el coeficiente de potencia del rotor se vería menos afectado.

En las siguientes figuras se muestran unos modelos tridimensionales del aspecto exterior de la pala, y cómo quedaría con un refuerzo circular o de sección cuadrada, con el extremo de pala macizo.

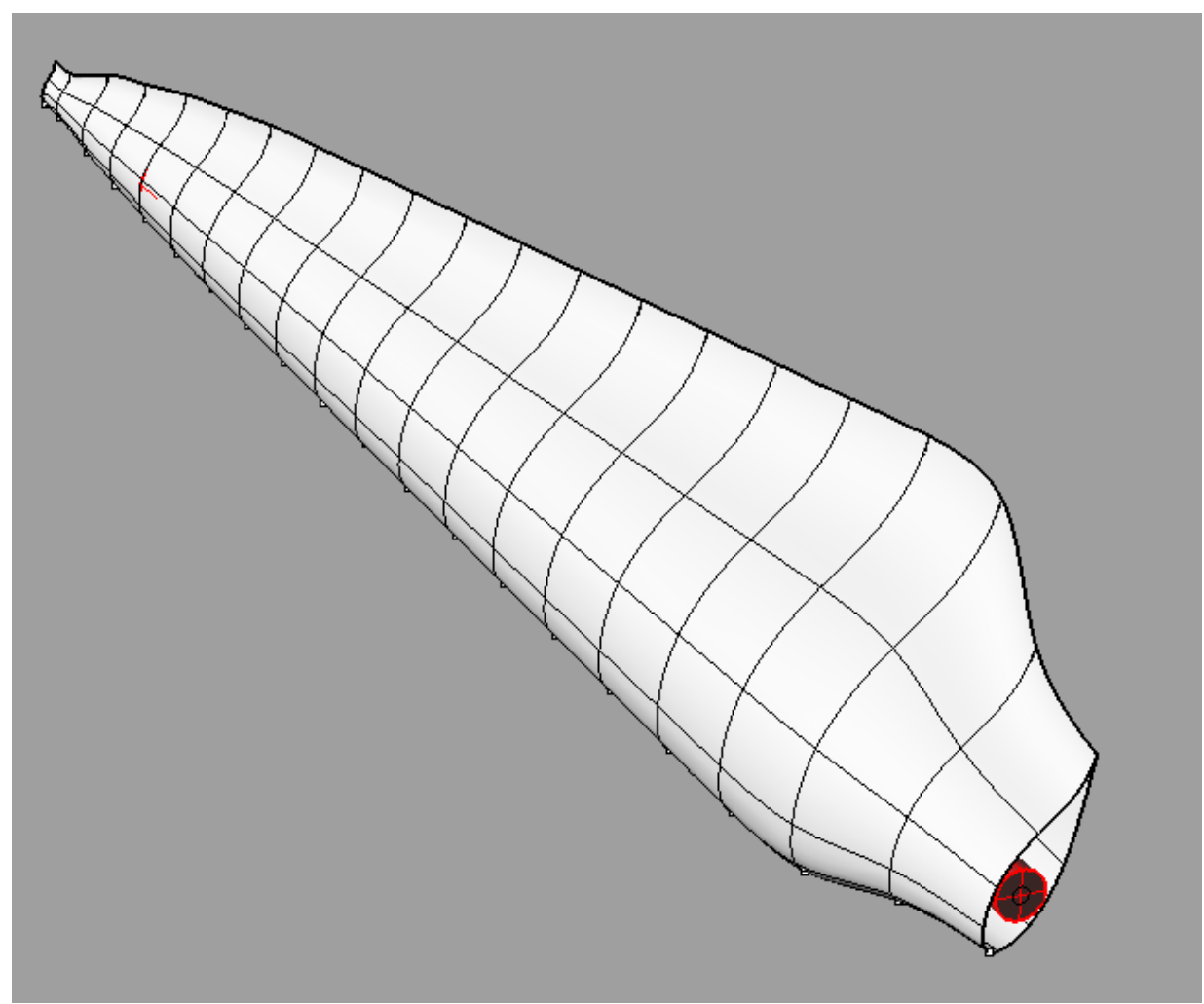

Figura 46. Aspecto de la pala en estudio 


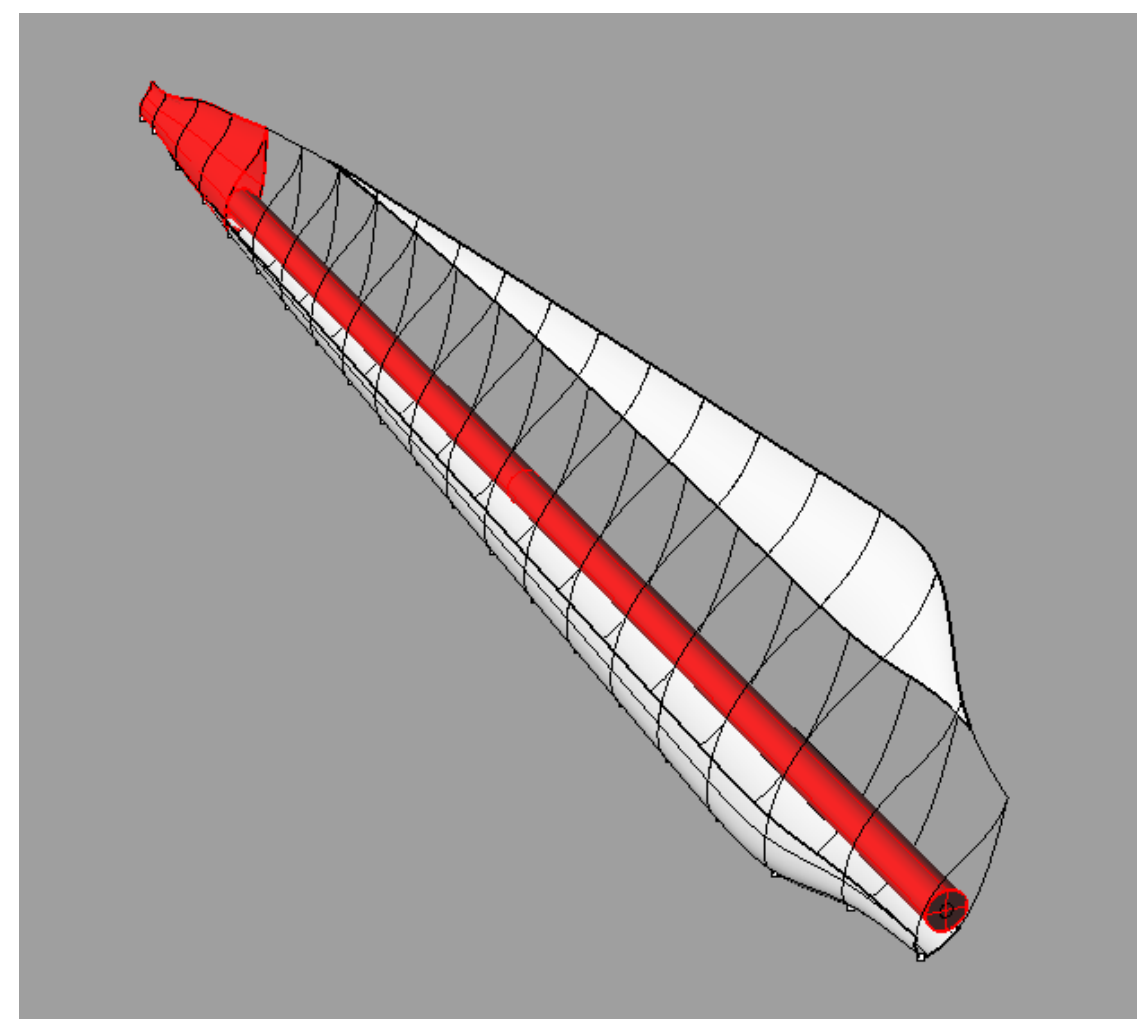

Figura 47. Estructura interior de la pala compuesta por un refuerzo principal circular y extremo de pala macizo

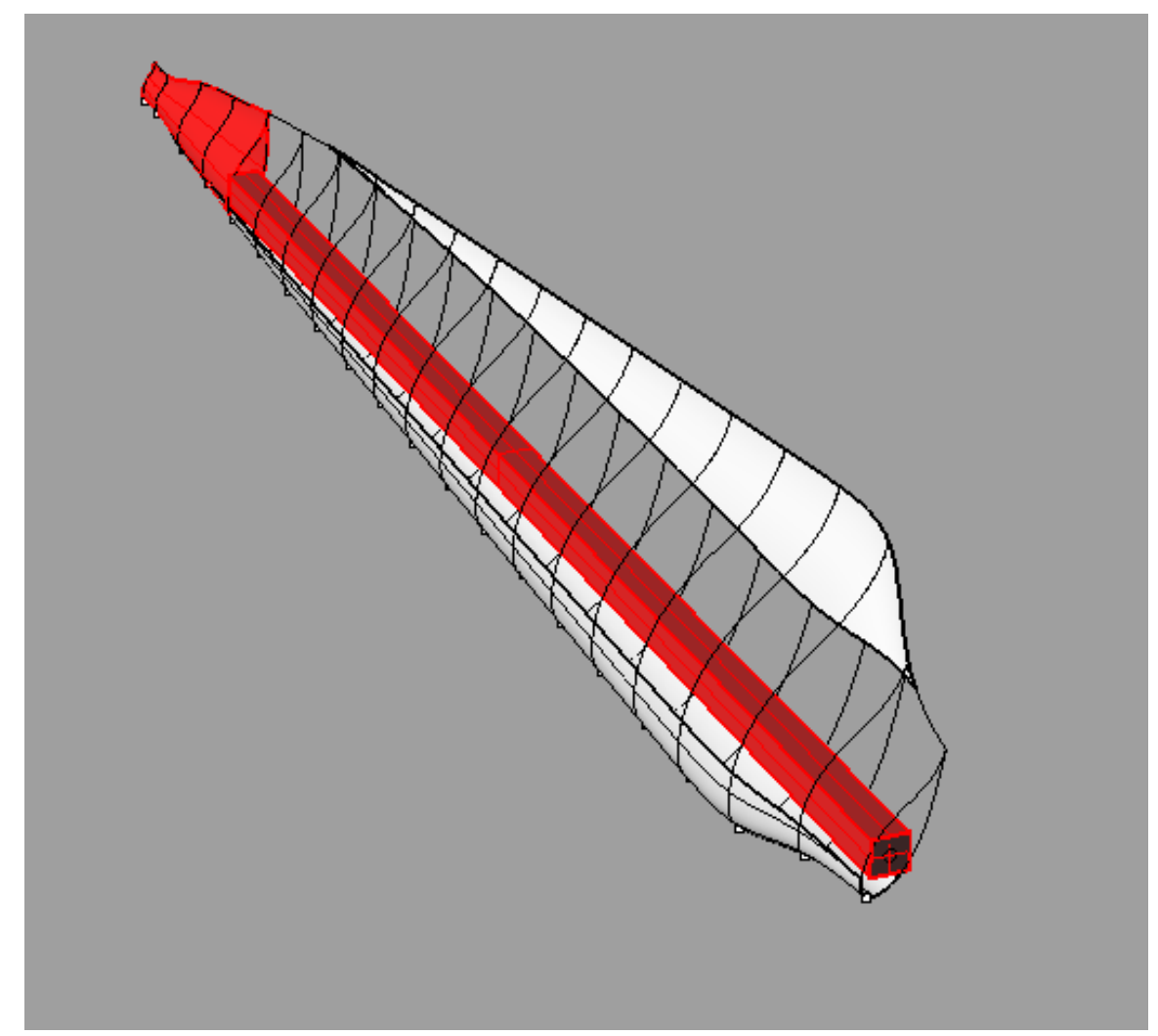

Figura 48. Estructura interior de la pala compuesta por un refuerzo de sección cuadrada y extremo de pala macizo 


\subsection{APLICACIÓN AL GESMEY $[60,61,62]$}

El GESMEY es un dispositivo que aprovecha la energía de las corrientes marinas y cuyo nombre responde a las siglas Generador Eléctrico Sub-Marino con Estructura en Y. De acuerdo a su denominación, el generador se compone de una turbina de corrientes instalada en el centro de la mencionada estructura con forma de $\mathrm{Y}$.

En los extremos de esta estructura, se disponen unos tanques de lastre dinámicos que permiten controlar el equilibrio hidrostático durante el funcionamiento, y también la maniobra de emersión para realizar trabajos de mantenimiento.

De esta forma, se simplifica la instalación y la operación, ya que solamente requiere unos puntos de amarre en el fondo marino y no es necesaria la construcción de estructuras fijas al fondo que soporten las solicitaciones a las que están sometidos dispositivos de aprovechamiento que funcionen sumergidos.

De las dos posibilidades de diseño de GESMEY: el GSY-A6.5 y el GSY U1M, de 600 kW y $1 \mathrm{MW}$ de potencia nominal [45]. Los parámetros de funcionamiento de cada uno de estos diseños del generador GESMEY se recogen en la Tabla 32, así como las características principales del rotor en cuanto a diámetro de éste, material de construcción, etc.

\begin{tabular}{lll}
\hline DESCRIPCION & GSY -A6.5 & GSY U1M \\
\hline Potencia $(\mathrm{kW})$ & 600 & 1.000 \\
\hline Velocidad máxima de corriente $(\mathrm{m} / \mathrm{s})$ & 2,0 & 1,8 \\
\hline Profundidad del suelo marino $(\mathrm{m})$ & $60 / 100$ & 80 \\
\hline Diámetro del rotor $(\mathrm{m})$ & 20 & 32 \\
\hline Profundidad mínima del extremo de pala $(\mathrm{m})$ & 15 & 34 \\
\hline Relación de multiplicación (RPM) & $12 / 1.500$ & $12 / 750$ \\
\hline Material estructural & Acero & Acero \\
\hline Numero de generadores por parque & $20 / 50$ & $20 / 50$ \\
\hline
\end{tabular}

Tabla 32. Parámetros principales de funcionamiento de los diseños GSY-A6.5 y GSY U1M [61]

Puesto que no se ha encontrado información detallada acerca de la geometría de pala, en cuanto a distribuciones de ángulos de paso geométrico, distribución de cuerdas, radio del núcleo del rotor y perfil bidimensional de cada sección de pala, se va a partir de unas distribuciones similares a las del apartado anterior [26]. Aunque luego durante el proceso de obtención de soluciones se vayan modificando ligeramente estas características geométricas.

La correcta elección de las distribuciones que definen la geometría de pala, es un aspecto crítico en el diseño de una turbina de corrientes, ya que pequeñas modificaciones pueden ocasionar una caída 
drástica del coeficiente de potencia esperado, así como de la potencia que el rotor entrega al eje de la turbina, que accionará el generador eléctrico.

\section{Perfil sustentador NACA 63-212}

Para ello, el perfil NACA 63-212 [11], aparte de existir documentos en los que se aporta gran cantidad de información acerca de sus características de funcionamiento, se trata de un perfil bidimensional bastante adecuado puesto que se ha utilizado en otros dispositivos de corrientes marinas. De este perfil se disponen de curvas de coeficientes de sustentación y arrastre, y curva de aparición de cavitación [46].

Para la distribución de cuerdas y ángulos de paso geométrico en la pala, se implementarán, en primera instancia, mismas distribuciones que se utilizaron en el apartado anterior, debido a que se trataba también de una turbina de 20 metros de diámetro, que es un caso similar a los de estudio en este apartado. A continuación, se presentan las tablas de y gráficas, de coeficientes de sustentación y de arrastre, así como la geometría del perfil y la distribución de aparición de cavitación.

\subsubsection{CÁLCULO DE LAS CARGAS INDUCIDAS EN LA PALA PARA EL GESMEY GSY-A6.5}

En este apartado se implementará el procedimiento de cálculo para obtener las cargas inducidas en la pala por la corriente fluida. Estas cargas se estiman con las expresiones de las ecuaciones Ec. 75 y Ec. 76, y para obtenerlas, es necesario llevar a cabo una serie de pasos.

En primer lugar hay que definir la geometría de pala, en segundo lugar hay que obtener las variables auxiliares para cada una de las secciones de pala y finalmente hay que determinar la distribución de ángulos de ataque. Un paso intermedio consiste en definir las distribuciones de coeficientes de sustentación y arrastre en función del ángulo de ataque.

En las Tabla 33 y Tabla 34 se muestran los parámetros de funcionamiento del rotor y la geometría de pala, que en este caso, se ha dividido la pala en 20 secciones al igual que en el caso estudiado en el apartado anterior y el perfil sustentador elegido de las secciones de pala es el NACA63-212 . De acuerdo a la Tabla 32, se estima una potencia entregada al eje del rotor de $600 \mathrm{~kW}$, para conseguir esta potencia, el coeficiente de potencia $c_{P}$ debe estar en torno 0,47 aproximadamente.

\begin{tabular}{|c|c|}
\hline rho $[\mathrm{kg} / \mathrm{m} 3]$ & 1025,34 \\
\hline $\mathbf{U}[\mathrm{m} / \mathrm{s}]$ & 2 \\
\hline $\mathbf{H}[\mathrm{m}]$ & 25 \\
\hline $\mathbf{Z}$ & 3 \\
\hline $\mathbf{r p m}$ & 12 \\
\hline $\mathbf{a}[\mathrm{m}]$ & 1 \\
\hline $\mathbf{b}[\mathrm{m}]$ & 10 \\
\hline nhu $[\mathrm{m} 2 / \mathrm{s}]$ & 0,000001306 \\
\hline
\end{tabular}

Tabla 33. Parámetros principales de funcionamiento del GSY-A6.5 implementados para el procedimiento de cálculo 


\section{Cálculo de las variables auxiliares y velocidad resultante $V_{k}$}

El siguiente paso a dar para implementar el procedimiento de cálculo, es calcular las funciones integrales $\lambda$ y $\mu$, y la función $\varphi$ para cada uno de los radios $r_{k}$. Como se ha comentado en apartados anteriores, estas variables solamente dependen del radio del núcleo del rotor $a$, el radio del extremo de pala $b$ y los radios de cada una de las secciones $r_{k}$.

Puesto que la configuración de radios $\mathrm{r}_{\mathrm{k}}$, el valor de $\mathrm{a}$ y $\mathrm{b}$ son idénticos que en el apartado 7.2 el valor de estas funciones auxiliares permanece invariable y se puede consultar la distribución de cada una de éstas en la Tabla 35. Así pues, con todas estas consideraciones se obtiene ya toda la información necesaria para comenzar el procedimiento de cálculo.

La velocidad resultante $\mathrm{V}_{\mathrm{k}}$ se obtiene con la ecuación Ec. 94, y puesto que depende de la velocidad de rotación, siempre que se quiera estudiar el comportamiento del rotor a distintas velocidades de rotación, hay que obtener de nuevo la distribución de velocidades resultantes.

\begin{tabular}{|c|c|c|c|c|c|}
\hline SECTION no & $\boldsymbol{r}_{\boldsymbol{k}}[\boldsymbol{m}]$ & $\boldsymbol{\delta}_{\boldsymbol{k}}[\boldsymbol{m}]$ & $\left.\boldsymbol{\beta}_{\boldsymbol{k}}{ }^{\circ}\right]$ & $\boldsymbol{c}_{\boldsymbol{k}}[\boldsymbol{m}]$ & perfil \\
\hline 1 & 1,225 & 0,45 & 36,90 & 0,950 & NACA 63-212 \\
\hline 2 & 1,675 & 0,45 & 42,90 & 1,150 & NACA 63-212 \\
\hline 3 & 2,125 & 0,45 & 48,90 & 1,550 & NACA 63-212 \\
\hline 4 & 2,575 & 0,45 & 54,90 & 1,600 & NACA 63-212 \\
\hline 5 & 3,025 & 0,45 & 60,90 & 1,550 & NACA 63-212 \\
\hline 6 & 3,475 & 0,45 & 64,90 & 1,500 & NACA 63-212 \\
\hline 7 & 3,925 & 0,45 & 66,90 & 1,450 & NACA 63-212 \\
\hline 8 & 4,375 & 0,45 & 68,90 & 1,400 & NACA 63-212 \\
\hline 9 & 4,825 & 0,45 & 69,90 & 1,350 & NACA 63-212 \\
\hline 10 & 5,275 & 0,45 & 70,90 & 1,300 & NACA 63-212 \\
\hline 11 & 5,725 & 0,45 & 71,90 & 1,250 & NACA 63-212 \\
\hline 12 & 6,175 & 0,45 & 72,90 & 1,200 & NACA 63-212 \\
\hline 13 & 6,625 & 0,45 & 73,90 & 1,150 & NACA 63-212 \\
\hline 14 & 7,075 & 0,45 & 74,90 & 1,100 & NACA 63-212 \\
\hline 15 & 7,525 & 0,45 & 75,90 & 1,050 & NACA 63-212 \\
\hline 16 & 7,975 & 0,45 & 76,40 & 0,975 & NACA 63-212 \\
\hline 17 & 8,425 & 0,45 & 76,90 & 0,900 & NACA 63-212 \\
\hline 18 & 8,875 & 0,45 & 76,90 & 0,800 & NACA 63-212 \\
\hline 19 & 9,325 & 0,45 & 76,90 & 0,700 & NACA 63-212 \\
\hline 20 & 9,775 & 0,45 & 76,90 & 0,550 & NACA 63-212 \\
\hline
\end{tabular}

Tabla 34. Geometría de pala definida para el GSY-A6.5 en 20 secciones

\begin{tabular}{|c|c|c|c|c|}
\hline SECTION no & $\boldsymbol{r}_{\boldsymbol{k}}[\boldsymbol{m}]$ & $\boldsymbol{\varphi}_{\boldsymbol{k}}$ & $\boldsymbol{\mu}\left(\boldsymbol{r}_{\boldsymbol{k}}\right)$ & $\boldsymbol{\lambda}\left(\boldsymbol{r}_{\boldsymbol{k}}\right)$ \\
\hline 1 & 1,225 & 1,47793 & 5,09 & 7,5992 \\
\hline 2 & 1,675 & 1,12337 & 0,78302 & 2,6613 \\
\hline
\end{tabular}




\begin{tabular}{|c|c|c|c|c|}
\hline SECTION no & $\boldsymbol{r}_{\boldsymbol{k}}[\boldsymbol{m}]$ & $\boldsymbol{\varphi}_{\boldsymbol{k}}$ & $\boldsymbol{\mu}\left(\boldsymbol{r}_{\boldsymbol{k}}\right)$ & $\boldsymbol{\lambda}\left(\boldsymbol{r}_{\boldsymbol{k}}\right)$ \\
\hline 3 & 2,125 & 0,88180 & 0,21016 & 1,5219 \\
\hline 4 & 2,575 & 0,72688 & 0,07124 & 1,017 \\
\hline 5 & 3,025 & 0,62628 & 0,027954 & 0,75136 \\
\hline 6 & 3,475 & 0,55940 & 0,011912 & 0,59847 \\
\hline 7 & 3,925 & 0,51450 & 0,005154 & 0,50593 \\
\hline 8 & 4,375 & 0,48492 & 0,00208 & 0,44931 \\
\hline 9 & 4,825 & 0,46689 & 0,00053 & 0,41393 \\
\hline 10 & 5,275 & 0,45833 & 0,00011 & 0,40133 \\
\hline 11 & 5,725 & 0,45833 & 0,00011 & 0,40133 \\
\hline 12 & 6,175 & 0,46689 & 0,00053 & 0,41393 \\
\hline 13 & 6,625 & 0,48492 & 0,00208 & 0,44931 \\
\hline 14 & 7,075 & 0,51450 & 0,005154 & 0,50593 \\
\hline 15 & 7,525 & 0,55940 & 0,011912 & 0,59847 \\
\hline 16 & 7,975 & 0,62628 & 0,027954 & 0,75136 \\
\hline 17 & 8,425 & 0,72688 & 0,07124 & 1,017 \\
\hline 18 & 8,875 & 0,88180 & 0,21016 & 1,5219 \\
\hline 19 & 9,325 & 1,12337 & 0,78302 & 2,6613 \\
\hline 20 & 9,775 & 1,47793 & 5,09 & 7,5992 \\
\hline
\end{tabular}

Tabla 35. Variables auxiliares $\lambda$ y $\mu$, y la función $\varphi$ para cada una de las secciones

\section{Cálculo de los ángulos de ataque $\gamma_{k}$ para la velocidad de rotación nominal $(\mathrm{rpm}=12)$}

El primer caso a estudiar, es la condición nominal de funcionamiento del rotor en el que éste entrega la potencia nominal para la generación de electricidad. La distribución de ángulos de ataque tiene que cumplir la condición de que el sumatorio de los valores $\psi_{\mathrm{k}}$, según la ecuación Ec. 116, sea aproximadamente cero, de acuerdo con el esquema del Apartado 4.1.7.1. Las ecuaciones planteadas admiten varias soluciones.

Una de las distribuciones de ángulos de ataque que cumple la condición expuesta, es suponer una distribución lineal decreciente a lo largo de la pala. En este caso la distribución lineal obtenida tiene un ángulo de ataque de $3,12^{\circ}$ en la sección del núcleo de pala y $0,2^{\circ}$ en la sección de extremo de pala.

Esta distribución lineal se construye otorgando valores tanteados los ángulos de ataque de las sección extremas de pala de forma que se anule el sumatorio de las variables auxiliares $\psi_{\mathrm{k}}$. Los ángulos de ataque obtenidos de esta forma se muestran junto los coeficientes de sustentación, arrastre y momento en la Tabla 36.

El coeficiente de potencia resultante de las cargas que se producen en la pala es igual a 0,445 y la potencia del rotor es de $576 \mathrm{~kW}$, valores algo inferiores a los esperados. El valor del sumatorio de $\psi_{\mathrm{k}}$ es 0,052 y la circulación $6,04 \mathrm{~m}^{2} / \mathrm{s}$. 
En la siguiente Tabla 36 se recogen los valores de las distribuciones de ángulos de ataque, coeficientes de sustentación y coeficientes de arrastre.

\begin{tabular}{|c|c|c|c|c|c|c|c|c|}
\hline no & r_k[m] & delta_k[m] & bheta_k[o] & c_k[m] & PERFIL & gamma_k[] & cl_k & cd_k \\
\hline 1 & 1,225 & 0,45 & 36,89 & 0,95 & NACA63-212 & 5,09 & 0,719 & 0,011 \\
\hline 2 & 1,675 & 0,45 & 42,89 & 1,15 & NACA63-212 & 4,84 & 0,696 & 0,01 \\
\hline 3 & 2,125 & 0,45 & 48,89 & 1,55 & NACA63-212 & 4,58 & 0,661 & 0,01 \\
\hline 4 & 2,575 & 0,45 & 54,89 & 1,6 & NACA63-212 & 4,32 & 0,619 & 0,01 \\
\hline 5 & 3,025 & 0,45 & 60,89 & 1,55 & NACA63-212 & 4,06 & 0,581 & 0,011 \\
\hline 6 & 3,475 & 0,45 & 64,9 & 1,5 & NACA63-212 & 3,81 & 0,548 & 0,011 \\
\hline 7 & 3,924 & 0,45 & 66,9 & 1,45 & NACA63-212 & 3,55 & 0,519 & 0,011 \\
\hline 8 & 4,375 & 0,45 & 68,9 & 1,4 & NACA63-212 & 3,29 & 0,494 & 0,011 \\
\hline 9 & 4,825 & 0,45 & 69,9 & 1,35 & NACA63-212 & 3,03 & 0,469 & 0,011 \\
\hline 10 & 5,275 & 0,45 & 70,9 & 1,3 & NACA63-212 & 2,77 & 0,445 & 0,011 \\
\hline 11 & 5,724 & 0,45 & 71,9 & 1,25 & NACA63-212 & 2,52 & 0,421 & 0,011 \\
\hline 12 & 6,174 & 0,45 & 72,9 & 1,2 & NACA63-212 & 2,26 & 0,395 & 0,011 \\
\hline 13 & 6,625 & 0,45 & 73,9 & 1,15 & NACA63-212 & 2 & 0,37 & 0,011 \\
\hline 14 & 7,075 & 0,45 & 74,9 & 1,1 & NACA63-212 & 1,74 & 0,344 & 0,011 \\
\hline 15 & 7,525 & 0,45 & 75,9 & 1,05 & NACA63-212 & 1,48 & 0,318 & 0,011 \\
\hline 16 & 7,974 & 0,45 & 76,4 & 0,975 & NACA63-212 & 1,23 & 0,291 & 0,011 \\
\hline 17 & 8,425 & 0,45 & 76,9 & 0,9 & NACA63-212 & 0,97 & 0,265 & 0,011 \\
\hline 18 & 8,875 & 0,45 & 76,9 & 0,8 & NACA63-212 & 0,71 & 0,237 & 0,011 \\
\hline 19 & 9,324 & 0,45 & 76,9 & 0,7 & NACA63-212 & 0,45 & 0,211 & 0,011 \\
\hline 20 & 9,775 & 0,45 & 76,9 & 0,55 & NACA63-212 & 0,2 & 0,183 & 0,011 \\
\hline
\end{tabular}

Tabla 36. Distribución de ángulos de ataque, coeficientes de sustentación y arrastre para la condición nominal de funcionamiento del GSY-A6.5, $\mathrm{rpm}=12$ y U $=2.0 \mathrm{~m} / \mathrm{s}$

Si se implementa esta geometría de pala en el método de cálculo basado en los elementos de pala se obtiene un coeficiente de potencia negativo. Pero si se incrementan los ángulos de paso geométrico en $10^{\circ}$, se mejora el coeficiente de potencia hasta el valor 0,471 y con una potencia de rotor de 606 $\mathrm{kW}$.

Con esta transformación de la geometría de pala se consigue unas condiciones de funcionamiento equivalentes según se calcule con el procedimiento o bien con el método basado en elementos de pala.

Implementado esta geometría de pala en el método elementos de pala se obtienen las siguientes distribuciones de ángulos de ataque, coeficientes de sustentación y arrastre.

\begin{tabular}{|c|c|c|c|c|c|c|c|c|}
\hline no & $\mathbf{r}[\mathbf{k}][\mathbf{m}]$ & delta[k][m] & bheta[k][0] & $\mathbf{c [ k ] [ m ]}$ & PERFIL & gamma[k][0] & cl[k] & cd[k] \\
\hline 1 & 1,225 & 0,45 & 46,89 & 0,95 & NACA63-212 & 2,89 & 0,456 & 0,011 \\
\hline 2 & 1,675 & 0,45 & 52,89 & 1,15 & NACA63-212 & 1,46 & 0,315 & 0,011 \\
\hline 3 & 2,125 & 0,45 & 58,89 & 1,55 & NACA63-212 & 0,19 & 0,183 & 0,011 \\
\hline 4 & 2,575 & 0,45 & 64,9 & 1,6 & NACA63-212 & 1,05 & 0,274 & 0,011 \\
\hline 5 & 3,025 & 0,45 & 70,9 & 1,55 & NACA63-212 & 2,67 & 0,435 & 0,011 \\
\hline 6 & 3,475 & 0,45 & 74,9 & 1,5 & NACA63-212 & 3,64 & 0,529 & 0,011 \\
\hline 7 & 3,924 & 0,45 & 76,9 & 1,45 & NACA63-212 & 3,67 & 0,532 & 0,011 \\
\hline
\end{tabular}




\begin{tabular}{|c|c|c|c|c|c|c|c|c|}
\hline $\mathbf{n} \mathbf{r}$ & $\mathbf{r}[\mathbf{k}][\mathbf{m}]$ & delta[k][m] & bheta[k][0] & $\mathbf{c}[\mathbf{k}][\mathbf{m}]$ & PERFIL & gamma[k][0] & $\mathbf{c l [ k ]}$ & $\mathbf{c d}[\mathbf{k}]$ \\
\hline 8 & 4,375 & 0,45 & 78,9 & 1,4 & NACA63-212 & 3,93 & 0,564 & 0,011 \\
\hline 9 & 4,825 & 0,45 & 79,9 & 1,35 & NACA63-212 & 3,72 & 0,538 & 0,011 \\
\hline 10 & 5,275 & 0,45 & 80,9 & 1,3 & NACA63-212 & 3,65 & 0,53 & 0,011 \\
\hline 11 & 5,724 & 0,45 & 81,9 & 1,25 & NACA63-212 & 3,68 & 0,533 & 0,011 \\
\hline 12 & 6,174 & 0,45 & 82,9 & 1,2 & NACA63-212 & 3,81 & 0,548 & 0,011 \\
\hline 13 & 6,625 & 0,45 & 83,9 & 1,15 & NACA63-212 & 4,03 & 0,577 & 0,011 \\
\hline 14 & 7,075 & 0,45 & 84,9 & 1,1 & NACA63-212 & 4,33 & 0,621 & 0,01 \\
\hline 15 & 7,525 & 0,45 & 85,9 & 1,05 & NACA63-212 & 4,7 & 0,681 & 0,01 \\
\hline 16 & 7,974 & 0,45 & 86,4 & 0,975 & NACA63-212 & 4,8 & 0,693 & 0,01 \\
\hline 17 & 8,425 & 0,45 & 86,9 & 0,9 & NACA63-212 & 4,93 & 0,705 & 0,01 \\
\hline 18 & 8,875 & 0,45 & 86,9 & 0,8 & NACA63-212 & 4,75 & 0,689 & 0,01 \\
\hline 19 & 9,324 & 0,45 & 86,9 & 0,7 & NACA63-212 & 4,6 & 0,663 & 0,01 \\
\hline 20 & 9,775 & 0,45 & 86,9 & 0,55 & NACA63-212 & 4,58 & 0,661 & 0,01 \\
\hline
\end{tabular}

Tabla 37. Distribución de ángulos de ataque, coeficientes de sustentación y arrastre para la condición nominal de funcionamiento del GSY-A6.5, rpm $=12$ y U $=2.0 \mathrm{~m} / \mathrm{s}$ (BET)

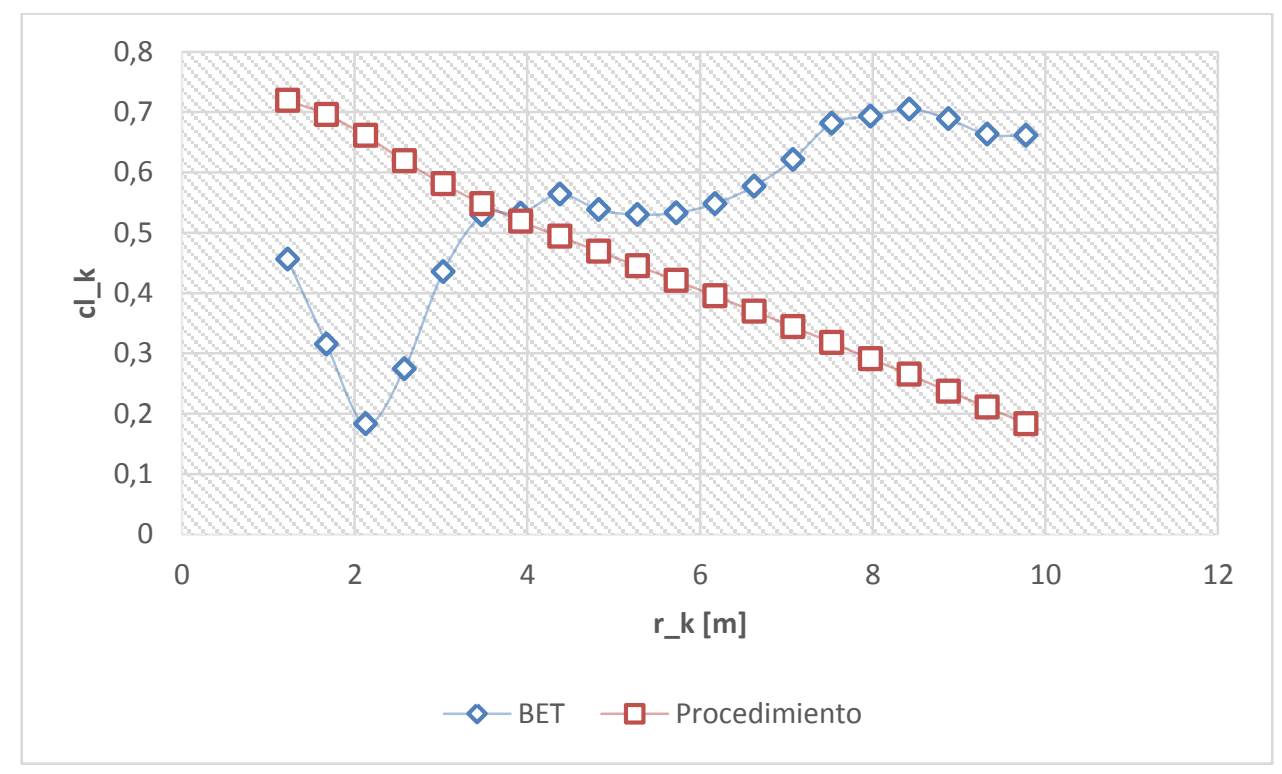

Figura 49. Gráfica comparativa de las distribuciones de coeficientes de sustentación a lo largo de la pala obtenidas con las distintas metodologías implementadas para rpm $=12, U=2 \mathrm{~m} / \mathrm{s}$

\section{Cargas resultantes en las secciones de pala}

Como se ha comentado al inicio de este apartado, las cargas en las secciones de pala se obtienen con las expresiones correspondientes a las ecuaciones Ec. 75 y Ec. 76. Puesto que ya se ha obtenido la distribución de ángulos de ataque, el resto de variables que intervienen en estas ecuaciones son conocidos y de este modo, se calculan directamente las distribuciones de estas cargas a lo largo de la pala. 
Puesto que las distribuciones de ángulos de ataque son distintas dependiendo de la metodología de cálculo utilizada, se pueden trazar gráficas comparativas con las distintas distribuciones de cargas a lo largo de la pala.

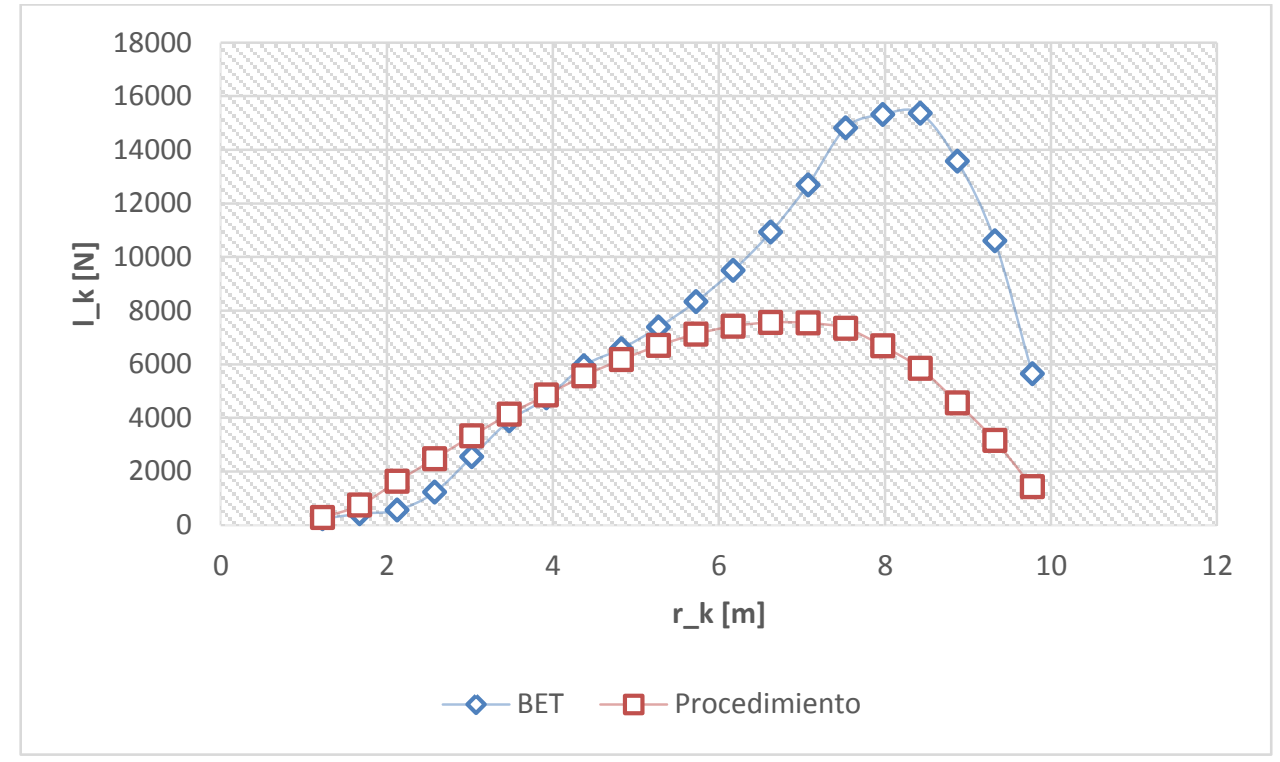

Figura 50. Gráfica comparativa de las distribuciones de cargas $l_{k}$ a lo largo de la pala obtenidas con las distintas metodologías implementadas para rpm $=12, U=2 \mathrm{~m} / \mathrm{s}$

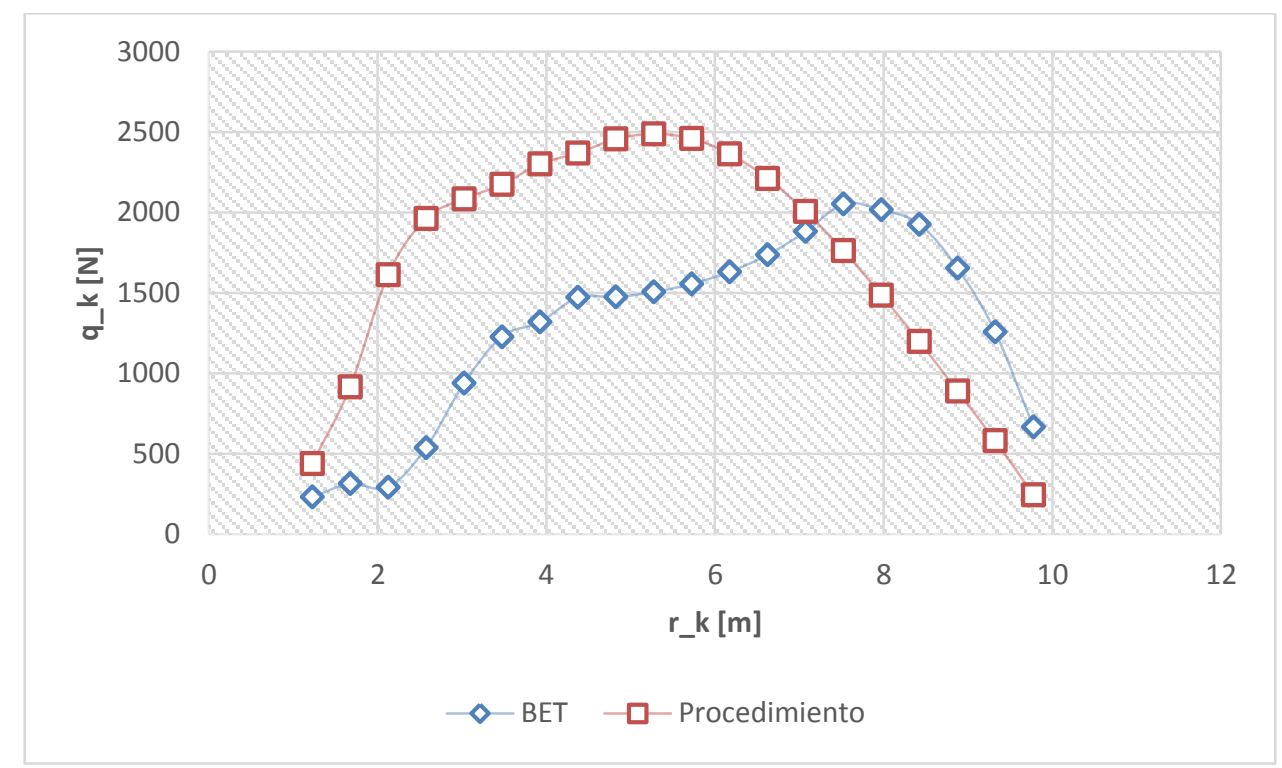

Figura 51. Gráfica comparativa de las distribuciones de cargas $\boldsymbol{q}_{\boldsymbol{k}}$ a lo largo de la pala obtenidas con las distintas metodologías implementadas para $\mathrm{rpm}=12, \mathrm{U}=2 \mathrm{~m} / \mathrm{s}$

\section{Momentos de Torsión}

El momento de torsión en la sección de pala tiene dos componentes, la primera de ellas corresponde a la establecida por la ecuación Ec. 16 y la torsión que sufre un perfil sustentador al moverse en una corriente fluida y que depende del coeficiente $c_{m}$, que se extrae de la curva polar del perfil bidimensional [11]. 
Si todas las fuerzas que actúan en la sección de pala estuviesen aplicadas en el mismo punto, entonces el momento de torsión de la sección sería igual al establecido en la mencionada ecuación.

Pero, en el caso de que las cargas $\mathrm{l}_{\mathrm{k}} \mathrm{y}_{\mathrm{k}}$ no se apliquen en el mismo punto que sus reacciones iguales y contrarias, se producirá un incremento del momento de torsión en la sección adicional.

Aunque el punto de aplicación de estas cargas varía con el ángulo de ataque, en principio, se puede considerar que este punto se encuentra aproximadamente a un tercio del borde de ataque de la sección y a la mitad del espesor de la sección en esa posición.

De esto se deduce que, para atenuar en la medida de posible este momento de torsión adicional, es importante que los centros estructurales de las secciones de pala estén lo más próximos al centro de aplicación de las fuerzas hidrodinámicas.

En la práctica conseguir esto no es tarea fácil ya que las secciones deben estar reforzadas longitudinalmente en toda su geometría para soportar los esfuerzos locales.

Así pues, para estudiar los momentos de torsión en la pala del rotor del GSY-A6.5 hay que suponer inicialmente una disposición estructural longitudinal. Con ésta, se obtendrá el correspondiente centro de gravedad y de este modo, como se ha comentado se obtiene el momento de torsión total en la sección.

Estos momentos se suman para obtener el momento de torsión total de la pala, y la distribución de momentos de torsión en la pala. Con la distribución de momentos de torsión en la pala se pueden calcular los ángulos de torsión que afectan a la geometría de pala y por tanto al rendimiento y funcionamiento del rotor.

Un diseño preliminar de la estructura de pala podría considerarse consistente en un refuerzo longitudinal aproximadamente rectangular. Este refuerzo atravesará todas las secciones desde el núcleo de pala hasta el extremo.

En este diseño preliminar se ha considerado el espesor de la pala. El conjunto de estos refuerzos longitudinales tendrá un centro de gravedad estructural en cada sección. Para el estudio de los ángulos de torsión en cada sección de pala se utilizarán los conceptos y ecuaciones del apartado 6 .

Por simplificación también se considera que la línea que forman el centro de gravedad estructural de la sección y el centro de aplicación de las fuerzas $\mathrm{l}_{\mathrm{k}}$ y $\mathrm{q}_{\mathrm{k}}$ forma el ángulo $\beta_{k}$ correspondiente al ángulo de paso geométrico de la sección. De esta forma el momento de torsión total en cada sección de pala se puede obtener con la siguiente expresión,

$$
Q_{k}=\frac{1}{2} \rho c_{m_{k}} c_{k}^{2} V_{k}^{2} \delta r_{k}+d_{k}\left(q_{k}+l_{k} \tan \beta_{k}\right)
$$

Siendo $\mathrm{d}_{\mathrm{k}}$ la distancia horizontal entre los puntos de aplicación de cargas hidrodinámicas y el centro de gravedad estructural de la sección. 
Las figuras correspondientes para el análisis de la torsión de las secciones de pala están recogidas en el ANEXO II de esta tesis.

\section{Cálculo de la constante geométrica $\mathrm{K}$ y del módulo de rigidez $G$}

La constante geométrica para el cálculo de la torsión, se obtiene a partir de las fórmulas de la Tabla 3 , que en este caso se puede asimilar el refuerzo longitudinal escogido a un rectángulo, lado menor igual a 0,076 m y de lado mayor 0,183 m, el espesor de estos lados es igual a 0,020 m.

Por tanto, la fórmula por la que se obtiene la constante geométrica $K$ es,

$$
K=\frac{2 \cdot 0,020^{2}(0,183-0,020)^{2}(0,076-0,020)^{2}}{0,183 \cdot 0,020+0,076 \cdot 0,020-2 \cdot 0,020^{2}}=1,5218 \cdot 10^{-5} m^{4}
$$

En los cálculos de torsión de la pala solamente se tendrá en cuenta el refuerzo principal, esto conlleva unos cálculos pesimistas ya que, de acuerdo al modelo estructural considerado, el material que recubre la estructura de la pala y cuyo espesor es de 0,01 metro, dificulta la torsión de la estructura, sin embargo, para poder tener en cuenta este aspecto sería necesario utilizar herramientas de cálculo por ordenador con mucha capacidad.

Es importante tener en cuenta el centro de gravedad de la sección de pala ya que es ahí donde se encuentran aplicadas las reacciones a las cargas hidrodinámicas.

En el caso de las secciones de pala estudiadas la posición de este centro conlleva a que las reacciones incrementen el momento hidrodinámico, pero según sea la disposición de los refuerzos puede ser que haya casos en el que el momento de torsión adicional al hidrodinámico se anule, disminuya a éste o bien sea de sentido contrario.

Una vez que se ha obtenido la constante geométrica $K$, para completar la relación entre los momentos de torsión y el ángulo de torsión de la ecuación Ec. 130, hay que obtener el módulo de rigidez $G$, que depende del material escogido para la construcción de los refuerzos ya que en su fórmula intervienen el módulo de Young $E$ y el de Poisson $v$.

Para el acero el módulo de rigidez es,

$$
G=\frac{E}{2(1+v)}=\frac{2100 \cdot 98066,5 \cdot 10^{3}}{2(1+0,25)}=8,23 \cdot 10^{10} \mathrm{~Pa}
$$

Para otros materiales se puede obtener el módulo de rigidez en base a la Tabla 38. 


\begin{tabular}{|c|c|c|}
\hline Material & $E\left(t / \mathrm{cm}^{2}\right)$ & $\mu$ \\
\hline Acero & 2.000 a 2.100 & 0.22 a 0.33 \\
\hline Cobre & 1.160 a 1.300 & 0.31 a 0.34 \\
\hline Bronce & 1.100 & 0.32 a 0.35 \\
\hline Hierro fundido & 750 a 1600 & 0.23 a 0.27 \\
\hline Aluminio & 760 & 0.32 a 0.36 \\
\hline Madera (paralela a la fibra) & 80 a 120 & - \\
\hline Hormigón & 150 a 350 & 0.10 a 0.20 \\
\hline Mampostería de ladrillo & $<120$ & - \\
\hline Caucho & 0.01 & 0.47 \\
\hline Corcho & - & $\approx 0.00$ \\
\hline
\end{tabular}

Tabla 38. Módulo de Young y Poisson para distintos materiales [54]

Con estos cálculos ya se puede obtener la distribución de momentos de torsión a lo largo de la pala del rotor, como el momento es acumulativo en las secciones de pala más cercanas al núcleo del rotor apenas se produce torsión y a medida que las secciones se van a cercando al extremo de pala el fenómeno de torsión se hace más evidente.

Puesto que la pala torsionada es una geometría diferente a la inicial, en la que ha variado los ángulos de paso $\beta_{k}$ geométricos de las secciones de pala, hay que recalcular la distribución de ángulos de ataque $\gamma_{k}$.

En principio, hasta que no se efectúen los cálculos no se puede saber si la torsión de la pala afecta positivamente o negativamente al coeficiente de potencia del rotor, ya que, como se ha dicho se considera que se trata de una geometría de pala en la que ha variado la distribución de ángulos de paso geométrico.

Si se calculase que la torsión de las secciones de pala produjese un efecto muy negativo sobre el coeficiente de potencia habría que considerar un cambio de los refuerzos longitudinales principales de forma que el centro de gravedad de las secciones se acercase lo máximo posible al centro de aplicación de las fuerzas hidrodinámicas.

Esta tarea, si se quiere realizar con precisión, se debe tener en cuenta que el centro de fuerzas hidrodinámicas varía su posición con el ángulo de ataque de la sección con la dificultada añadida de que el ángulo de ataque que interviene en cada sección no se puede conocer hasta que no se hayan efectuado los cálculos. De este modo, habría que realizar varias iteraciones con la misma metodología de estudio de la torsión de cada sección. 
A continuación se muestra en la Tabla 39 las distribuciones de momentos de torsión, los ángulos de torsión y en la última columna de la derecha la nueva distribución de ángulos de paso geométrico. En este caso el material de construcción de la pala es el acero y cuyo módulo de rigidez se calculó anteriormente.

Como se puede comprobar el efecto de la torsión según la disposición de refuerzos no es muy grande y el efecto sobre el coeficiente de potencia es de una disminución de en torno al $1 \%$.

\begin{tabular}{|c|c|c|c|c|c|c|c|}
\hline SECTION no & $\boldsymbol{r}_{\mathbf{k}} \mathbf{k}[\mathbf{m}]$ & $\boldsymbol{c}_{\boldsymbol{m}}$ & $\left.\boldsymbol{\gamma}_{\boldsymbol{k}}{ }^{\circ}\right]$ & $\boldsymbol{Q}_{\boldsymbol{k}}$ & $\sum_{\boldsymbol{i}}^{\boldsymbol{n}} \boldsymbol{Q}_{\boldsymbol{k}}[\mathbf{N m}]$ & $\boldsymbol{\theta}_{\boldsymbol{k}}\left[^{\circ}\right]$ & $\left.\boldsymbol{\beta}^{\prime}{ }{ }^{\circ}\right]$ \\
\hline 1 & 1,225 & $-0,0286$ & 3,12 & 85,770 & 85,770 & 0,005 & 36,90 \\
\hline 2 & 1,675 & $-0,0294$ & 2,9663158 & 143,170 & 228,940 & 0,018 & 42,92 \\
\hline 3 & 2,125 & $-0,0301$ & 2,8126316 & 319,610 & 548,550 & 0,053 & 48,95 \\
\hline 4 & 2,575 & $-0,0309$ & 2,6589474 & 449,470 & 998,020 & 0,118 & 55,02 \\
\hline 5 & 3,025 & $-0,0317$ & 2,5052632 & 637,960 & 1635,980 & 0,227 & 61,13 \\
\hline 6 & 3,475 & $-0,0322$ & 2,3515789 & 732,320 & 2368,300 & 0,377 & 65,28 \\
\hline 7 & 3,925 & $-0,0327$ & 2,1978947 & 838,620 & 3206,920 & 0,577 & 67,48 \\
\hline 8 & 4,375 & $-0,0332$ & 2,0442105 & 1195,430 & 4402,350 & 0,882 & 69,78 \\
\hline 9 & 4,825 & $-0,0337$ & 1,8905263 & 1617,190 & 6019,540 & 1,330 & 71,23 \\
\hline 10 & 5,275 & $-0,0343$ & 1,7368421 & 1397,950 & 7417,490 & 1,792 & 72,69 \\
\hline 11 & 5,725 & $-0,0348$ & 1,5831579 & 1360,070 & 8777,560 & 2,302 & 74,20 \\
\hline 12 & 6,175 & $-0,0351$ & 1,4294737 & 1205,420 & 9982,980 & 2,823 & 75,72 \\
\hline 13 & 6,625 & $-0,0354$ & 1,2757895 & 1231,420 & 11214,400 & 3,403 & 77,30 \\
\hline 14 & 7,075 & $-0,0359$ & 1,1221053 & 1231,530 & 12445,930 & 4,033 & 78,93 \\
\hline 15 & 7,525 & $-0,0363$ & 0,9684211 & 1261,660 & 13707,590 & 4,724 & 80,62 \\
\hline 16 & 7,975 & $-0,0366$ & 0,8147368 & 1215,340 & 14922,930 & 5,451 & 81,85 \\
\hline 17 & 8,425 & $-0,0368$ & 0,6610526 & 1171,150 & 16094,080 & 6,210 & 83,11 \\
\hline 18 & 8,875 & $-0,0371$ & 0,5073684 & 1013,250 & 17107,330 & 6,954 & 83,85 \\
\hline 19 & 9,325 & $-0,0375$ & 0,3536842 & 918,540 & 18025,870 & 7,699 & 84,60 \\
\hline 20 & 9,775 & $-0,0378$ & 0,2 & 659,740 & 18685,610 & 8,366 & 85,27 \\
\hline & & & & & & & \\
\hline
\end{tabular}

Tabla 39. Distribución de los momentos de torsión y ángulos de torsión en la pala

Por ello en este diseño propuesto del GESMEY GSY-A6.5 construido con acero, se puede estimar que el efecto de la torsión de las palas durante el funcionamiento del rotor es despreciable.

Si se obtiene el módulo de rigidez para el aluminio que según los datos de la Tabla 38 es igual a $2,8019 \cdot 10^{10} \mathrm{~Pa}$, se puede obtener la distribución de ángulos de torsión sobre las secciones de pala. Como la disposición de refuerzos se mantiene constante, la constante geométrica $\mathrm{K}$ mantiene el mismo valor que para el caso anterior.

Con estos datos se obtienen las distribuciones de momentos de torsión a lo largo de la pala, que se muestran en la Tabla 40. 
En este caso se hace más evidente el efecto de la torsión, sobre todo en las secciones de cercanas al extremo de pala donde se sobrepasan los $90^{\circ}$ para el ángulo de paso geométrico.

Hay que tener en cuenta la variación del coeficiente de potencia y recalcular la distribución de ángulos de ataque, ya que la geometría varía bastante y puede darse el caso de que la geometría con torsión dé lugar a distribuciones incompatibles con el procedimiento de cálculos o con el límite teórico de Betz.

\begin{tabular}{|c|c|c|c|c|c|c|c|}
\hline SECTION no & $\mathbf{r}_{\mathbf{k}} \mathbf{k}[\mathrm{m}]$ & $\boldsymbol{c}_{\boldsymbol{m}}$ & $\boldsymbol{\gamma}_{\boldsymbol{k}}{ }^{\left.{ }^{\circ}\right]}$ & $\boldsymbol{Q}_{\boldsymbol{k}}$ & $\sum_{\boldsymbol{i}=1}^{\boldsymbol{n}} \boldsymbol{Q}_{\boldsymbol{k}}[\mathbf{N m}]$ & $\boldsymbol{\theta}_{\boldsymbol{k}}\left[^{\circ}\right]$ & $\left.\boldsymbol{\beta}_{\boldsymbol{k}}{ }^{\circ}{ }^{\circ}\right]$ \\
\hline 1 & 1,225 & $-0,0286$ & 3,12 & 85,770 & 85,770 & 0,014 & 36,91 \\
\hline 2 & 1,675 & $-0,0294$ & 2,9663158 & 143,170 & 228,940 & 0,052 & 42,95 \\
\hline 3 & 2,125 & $-0,0301$ & 2,8126316 & 319,610 & 548,550 & 0,157 & 49,06 \\
\hline 4 & 2,575 & $-0,0309$ & 2,6589474 & 449,470 & 998,020 & 0,346 & 55,25 \\
\hline 5 & 3,025 & $-0,0317$ & 2,5052632 & 637,960 & 1635,980 & 0,666 & 61,57 \\
\hline 6 & 3,475 & $-0,0322$ & 2,3515789 & 732,320 & 2368,300 & 1,107 & 66,01 \\
\hline 7 & 3,925 & $-0,0327$ & 2,1978947 & 838,620 & 3206,920 & 1,693 & 68,59 \\
\hline 8 & 4,375 & $-0,0332$ & 2,0442105 & 1195,430 & 4402,350 & 2,591 & 71,49 \\
\hline 9 & 4,825 & $-0,0337$ & 1,8905263 & 1617,190 & 6019,540 & 3,907 & 73,81 \\
\hline 10 & 5,275 & $-0,0343$ & 1,7368421 & 1397,950 & 7417,490 & 5,264 & 76,16 \\
\hline 11 & 5,725 & $-0,0348$ & 1,5831579 & 1360,070 & 8777,560 & 6,760 & 78,66 \\
\hline 12 & 6,175 & $-0,0351$ & 1,4294737 & 1205,420 & 9982,980 & 8,293 & 81,19 \\
\hline 13 & 6,625 & $-0,0354$ & 1,2757895 & 1231,420 & 11214,400 & 9,995 & 83,89 \\
\hline 14 & 7,075 & $-0,0359$ & 1,1221053 & 1231,530 & 12445,930 & 11,846 & 86,75 \\
\hline 15 & 7,525 & $-0,0363$ & 0,9684211 & 1261,660 & 13707,590 & 13,877 & 89,78 \\
\hline 16 & 7,975 & $-0,0366$ & 0,8147368 & 1215,340 & 14922,930 & 16,011 & 92,41 \\
\hline 17 & 8,425 & $-0,0368$ & 0,6610526 & 1171,150 & 16094,080 & 18,241 & 95,14 \\
\hline 18 & 8,875 & $-0,0371$ & 0,5073684 & 1013,250 & 17107,330 & 20,425 & 97,33 \\
\hline 19 & 9,325 & $-0,0375$ & 0,3536842 & 918,540 & 18025,870 & 22,613 & 99,51 \\
\hline 20 & 9,775 & $-0,0378$ & 0,2 & 659,740 & 18685,610 & 24,572 & 101,47 \\
\hline
\end{tabular}

Tabla 40. Distribución de los momentos de torsión y ángulos de torsión en la pala considerando una estructura de aluminio 


\section{CONCLUSIONES}

En el ámbito de las energías renovables marinas existen numerosos DAECS (Dispositivos de Aprovechamiento de Energía de Corrientes Submarinas) que permiten transformar la energía cinética del movimiento de masas de agua en energía eléctrica.

Dentro de este conjunto, las turbinas de eje horizontal con rotor abierto son probablemente el dispositivo más adecuado para llevar a cabo esta transformación de energía. Prueba de ello son el gran número de publicaciones científicas, en los últimos años, dedicadas a las turbinas marinas de eje horizontal.

Esto se debe en gran medida, a que tienen un funcionamiento análogo al de las turbinas eólicas y que parte de la tecnología la desarrollada en esta últimas se puede utilizar también en el medio marino, sobre todo en áreas geográficas donde existen corrientes marinas cuya velocidad se mantiene casi constante a lo largo del año e independientemente de la meteorología, y también en zonas donde la geografía permite la instalación de DACES para el aprovechamiento de las corrientes de agua originadas por las mareas.

Por todo esto, el estudio del funcionamiento de las turbinas de corrientes marinas de eje horizontal es de gran importancia tanto a nivel teórico como a nivel experimental.

El cálculo de las cargas hidrodinámicas que se producen en la pala de un rotor de una turbina marina de eje horizontal, se ha efectuado en numerosas publicaciones, mediante métodos de cálculo basados en la Teoría de Elementos de Pala. Esta Teoría se empezó a utilizar en los inicios de la generación de energía eólica y se ha desarrollado con múltiples correcciones o complementos hasta nuestros días.

Los problemas básicos inherentes a los métodos de cálculo basados en esta teoría son por un lado, que no se tiene en cuenta los efectos de la interacción entre los distintos elementos de pala, y por otro lado, dependiendo de la geometría de pala escogida, pueden aparecer problemas de convergencia a la hora de determinar los factores de inducción axial y radial.

Y es en este ámbito donde hay que trabajar para establecer un modelo tridimensional que sea compatible con la Teoría de Elementos de Pala, y de este modo tratar de aportar una relación tridimensional de los distintos elementos de pala, con el objeto de complementar a esta Teoría.

El modelo tridimensional se fundamenta en la hipótesis de que la pala del rotor está inmersa en una línea de vórtice de intensidad de circulación constante [39]. Esta línea al ser seccionada por un plano situado en la estela de la pala originaría dos vórtices contrarrotativos.

Con esta hipótesis se define un volumen de control que contiene a la pala y con él se desarrollan las ecuaciones de volumen de control: Balance masa, balance de momento lineal y balance de energía.

Las ecuaciones obtenidas con el desarrollo anterior permiten calcular las fuerzas globales a las que está sometida la pala, así como la circulación de los vórtices contrarrotativos. 
Finalmente, se combinan estas expresiones con la Teoría de Elementos de Pala para obtener un procedimiento o método de cálculo.

En definitiva, el procedimiento de cálculo consiste, en esencia, en la aplicación de la Teoría de Elementos de Pala, pero incluyendo la intensidad de circulación $\Gamma$ que relaciona todas las secciones de pala.

Para que las ecuaciones planteadas tengan soluciones físicas con sentido, es necesario considerar unas determinadas distribuciones de velocidades del fluido aguas abajo ( $U^{*}$ y $v_{\omega r}^{*}$ ), que serán desconocidas, y que tendrán que determinarse como paso intermedio del esquema de ecuaciones a resolver.

Estas velocidades del fluido a la salida de las proximidades de la pala, contabilizan el cambio de velocidad de las partículas fluidas cuando pasan a través de la pala y así este intercambio de energía cinética se recoge en balance de energía global.

El módulo del vector velocidad en las inmediaciones de la sección de pala es igual al obtenido con la metodología de los elementos de pala, solamente variará el ángulo de ataque de las secciones con respecto a los que se obtendrían con los elementos de pala.

También se introduce en el procedimiento, expresiones para contabilizar las pérdidas por punta de pala y extremo de núcleo, así como ecuaciones adicionales para obtener los coeficientes de sustentación y arrastre para valores de ángulo de ataque superiores al ángulo de pérdida.

Con todo este desarrollo teórico se han confeccionado aplicaciones informáticas que implementan, tanto el procedimiento propuesto en esta tesis como el método de cálculo de elementos de pala. En estas aplicaciones se han desarrollado rutinas que permiten definir geometrías de pala complejas de forma relativamente cómoda.

La extrapolación de la Teoría de Elementos de Pala de las turbinas eólicas a las turbinas de corrientes marinas, tiene el inconveniente de que en las turbinas eólicas no existe el fenómeno de la cavitación, de ahí que sea necesario definir alguna corrección o complemento a los elementos de pala para mejorar este aspecto.

En este aspecto, al conseguir mejorar la predicción de los ángulos de ataque de las secciones de pala mediante la interacción tridimensional de estos elementos se puede mejorar la estimación de aparición de la cavitación en alguna zona concreta de la pala.

Los elementos estructurales que componen la pala del rotor están sometidos a tracción, flexión y torsión. De estos tres tipos de cargas, la más crítica desde el punto de vista del criterio del coeficiente de potencia es la torsión, ya que modifica los ángulos de paso geométrico de la pala y que están directamente relacionados con los ángulos de ataque.

El estudio de la torsión se plantea desde el punto de vista de los elementos de pala, es decir, se calculan las cargas a las que están sometidas cada una de las secciones de pala, que se han obtenido 
con el procedimiento de cálculo y se supone que se aplican en un punto situado aproximadamente a un tercio de la cuerda.

El momento de torsión tiene dos componentes, una que es debida al momento o par derivado de los elementos de pala y que dependen del coeficiente adimensional $c_{m}$ y otra que depende del punto de aplicación de las cargas.

La posición de este punto de aplicación de fuerzas es crítica para el efecto de torsión de la estructura de la pala, ya que cuanto más alejado esté este punto del centro de gravedad de la sección estructural mayor será el momento adicional de torsión.

Aunque en el método que se aplicado en la tesis se ha despreciado este efecto, sí que hay que tener en cuenta, con el objeto de realizar cálculos más precisos, que este punto de aplicación de las cargas hidrodinámicas varía con el ángulo de ataque.

El criterio más extendido para evaluar la bondad del funcionamiento de una turbina de corrientes, consiste en la valoración y cálculo del coeficiente de potencia, y en este aspecto, el efecto de la torsión afecta negativamente, por lo que hay que tratar de disponer los elementos estructurales de forma que el centro de gravedad de las secciones de pala, estén lo más cerca posible de los centros de aplicación de las cargas hidrodinámicas.

Este aspecto puede ser muy complejo de calcular, ya que hay que tener en cuenta que, como se ha comentado, la posición del centro de aplicación de las cargas hidrodinámicas varía con el ángulo de ataque. En esta tesis se aporta un método para abordar esta problemática de forma sencilla para poder estimar o tener un criterio global que permita discernir si una determinada configuración de refuerzos estructurales mejora o empeora las deformaciones torsionales.

De las distintas implementaciones de las ecuaciones del procedimiento de cálculo, efectuadas en el apartado 7 , se puede concluir, de los resultados obtenidos que las geometrías de pala que tienen ángulos de paso geométrico muy próximos a $90^{\circ}$ dan lugar a coeficientes de potencia muy bajos, y al disminuir estos ángulos ligeramente se mejora la obtención de resultados.

También se ha comprobado que algunas geometrías de pala en las que el ángulo de paso geométrico está cercano a $75^{\circ}$, tienen coeficientes de potencia con valores muy bajos desde el punto de vista de la Teoría de Elementos de Pala.

De este hecho, se puede establecer que las geometrías de pala que tengan ángulos de paso geométrico en el extremo de pala en torno a los $75^{\circ}$ aproximadamente, tendrían mejores resultados debido a las interacciones de las distintas secciones de pala.

El coeficiente de potencia en los casos en que el extremo de pala tiene un ángulo de paso geométrico de $75^{\circ}$ aproximadamente alcanza valores cercanos entre 0,4 y 0,5 según los resultados de las ecuaciones del procedimiento de cálculo propuesto en esta tesis.

El primer caso de implementación se basa en un ejemplo incluido en un documento dedicado a la Teoría de Elementos de Pala. En este caso el rotor en estudio pertenece a una turbina eólica, sin 
embargo, los resultados de este ejemplo son de gran utilidad ya que permiten depurar errores del programa de cálculo confeccionado.

Puesto que los fundamentos teóricos se basan en la Teoría de Elementos de pala, es fundamental contar con una rutina de cálculo que proporcione resultados correctos. Ya que, para la implementación de las ecuaciones del procedimiento alternativo de esta tesis, es necesario también calcular de forma precisa los factores de inducción axial y radial para obtener el módulo de la velocidad del fluido en el entorno cercano de las secciones de pala.

En el segundo caso de implementación, se estudia una turbina con un diámetro de 1 metro y con ángulo de paso geométrico de $88^{\circ}$ y cuerda constantes a lo largo de toda la pala. Y en este caso el coeficiente de potencia obtenido con el método de elementos de pala es inferior al esperado.

Se comprueba también que las ecuaciones del procedimiento alternativo de esta tesis con la geometría de pala inicial obtiene un coeficiente de potencia muy bajo, y sin embargo, reduciendo el ángulo de paso geométrico hasta $60^{\circ}$, se obtienen unos resultados equivalentes a los calculados con el método de elementos de pala.

La distribución de cargas $l_{k}$ deducidas del método de elementos de pala presenta un crecimiento abrupto en la zona de extremo de pala, mientras que éste desaparece en la distribución de estas mismas cargas calculadas con las ecuaciones de procedimiento de esta tesis.

Una vez realizadas implementaciones preliminares, se estudia el primer caso de turbina de corrientes marinas. En este primer caso, se corrobora este hecho con el método de elementos de pala, ya que, se obtienen prácticamente los mismos resultados aplicando a la misma geometría de pala.

Al igual que ocurría con el caso anterior, con la geometría inicial se obtiene un coeficiente de potencia muy bajo al aplicar el esquema de cálculo propuesto. Sin embargo, disminuyendo ligeramente los ángulos de paso geométrico se obtienen resultados equivalentes a los calculados con el método de elementos de pala, y también esta nueva geometría resultante funciona bien con este método.

Con el segundo caso de implementación, ocurre lo mismo, puesto que la geometría de pala está definida de forma que el extremo de pala tiene un ángulo cercano a $90^{\circ}$, el coeficiente de potencia calculado inicialmente con el procedimiento, presenta un valor muy bajo y muy cercano al esperado, con la implementación del método de elementos de pala. Sin embargo, ocurre lo contrario al reducir el ángulo de paso geométrico de las secciones de pala en $16^{\circ}$, el coeficiente de potencia obtenido con el método de elementos de pala se reduce considerablemente, y según el procedimiento se obtiene un valor de este coeficiente bastante bueno.

El hecho de que existan geometrías que presenten resultados pesimistas con la aplicación del método de elementos de pala, hay que tenerlo en cuenta a la hora de estudiar diferentes geometrías de pala, ya que se pueden definir formas muy complejas y parece obvio que existan algunas que tengan mejores o peores resultados, tanto desde la aplicación directa de los elementos de pala, como de las relaciones establecidas en esta tesis para trata de valorar la interacción tridimensional de los distintos elementos de pala. 
Lo mismo ocurre para el último caso implementado, a partir de la geometría de pala inicial para la que el método de elementos de pala ha dado resultados correctos, el coeficiente de potencia obtenido con el procedimiento es muy bajo. Y sin embargo, al igual que para los caso anteriores, si se aplica una reducción de los ángulos de paso geométrico de las secciones se obtienen unos resultados equivalentes a los obtenidos con el método de elementos de pala.

En los numerosos procesos de cálculo llevados a cabo para confeccionar este apartado 7, se han detectado varios problemas a resolver en futuros trabajos. El principal de ellos consiste en que, aunque los resultados son buenos para velocidades TSR iguales o inferiores a la de funcionamiento nominal, sin embargo, para valores superiores el coeficiente de potencia crece y los resultados se distancian de los obtenidos con el método de elementos de pala.

Este problema puede tener varios orígenes, uno de ellos parte de la resolución de las ecuaciones del procedimiento que, en esta tesis, como metodología preliminar se ha optado por utilizar distribuciones de ángulos de ataque lineales a lo largo de toda la pala. Y en este sentido habría que elaborar una metodología compleja para la búsqueda de soluciones partiendo de la distribución de ángulos de ataque calculada con el método de elementos de pala.

El crecimiento del coeficiente de potencia para determinadas velocidades TSR elevadas, se podría solucionar con la deducción de una expresión de este coeficiente que dependa de la variable TSR, al igual que existe para el método de elementos de pala.

Otro problema es el cálculo de coeficientes de potencia muy bajos con las ecuaciones del procedimiento para determinadas geometrías de pala, en este aspecto habría que hacer un análisis minucioso para poder determinar qué parámetros de la geometría de pala afectan a este cálculo de forma determinante. Aunque hay que tener en cuenta que este hecho también sucede con la aplicación de la Teoría de Elementos de Pala.

También las ecuaciones del procedimiento se pueden incorporar a la Teoría de Elementos de Pala como condición adicional que permita solucionar los posibles problemas de convergencia a la hora de calcular los coeficientes de inducción axial y radial.

El replanteamiento del procedimiento de forma que el módulo de la velocidad $\mathrm{V}$ se obtenga a partir de los teóricos coeficientes de inducción axial y radial calculados directamente con la distribución de ángulos de ataque solución de las ecuaciones es un aspecto importante para seguir la línea de trabajo comenzada en esta tesis.

Un aspecto conceptual muy importante, que se deduce de los resultados obtenidos, es que habría que comprobar con una herramienta de cálculo basada en CFD, si efectivamente las geometrías de pala con ángulos de paso geométrico con valores de aproximadamente $75^{\circ}$ tienen mejor coeficiente de potencia.

El planteamiento y desarrollo teórico llevado a cabo constituye una idea original sobre la que hay que seguir trabajando, para que se consiga obtener en el futuro una herramienta de criterio que permita saber qué modificaciones de geometría de pala van a dar mejores o peores resultados en la realidad. 
Mejorar y complementar la Teoría de Elementos de Pala es un objetivo que se ha comprobado que es necesario abordar, ya que es una herramienta de gran utilidad para el diseño básico de geometrías de pala, pero tiene algunos aspectos a mejorar, uno de ellos es el despreciar la interacción tridimensional de los elementos de pala, y otro, como se ha visto en los resultados obtenidos, es que es un método muy rígido que con ligeras variaciones de la geometría de pala se empeoran demasiado los resultados, y todo esto aparte de los posibles problemas de convergencia de los factores de inducción axial y radial que se han comentado anteriormente.

El desarrollo de ideas y planteamientos originales que sean compatibles con la Teoría de Elementos de Pala, es fundamental para mejorar esta teoría, y adaptarla al ámbito de la turbinas de corrientes marinas. Y esta tesis pretende prestar su aportación a trabajos futuros en esta incipiente línea de investigación. 


\section{BIBLIOGRAFÍA}

[1] Shujie Wang, Lanshuai Lu, Dong Li, Zhongde Shan and Longwu Zhao. Experimental Study on Flexible Bladeturbine Driven by Tidal Current Energy. Chinese-German Joint Symposium on Hydraulic and Ocean Engineering, August 24-30, Darmstadt, Alemania (2008).

[2] Paul A. Lynn. An Introduction to Marine Energy. 1a edición. Londres. John Wiley \& Sons Ltd. (2014).

[3] Magdi Ragheb and Adam M. Ragheb. Wind Turbines Theory - The Betz Equation and Optimal Rotor Tip Speed Ratio, Fundamental and Advanced Topics in Wind Power. (1a edición). Dr. Rupp Carriveau (Ed.). ISBN: 978-953-307-508-2. InTech. (2011).

[4] Mg. Amancio R. Rojas Flores. Fundamentos Aerodinámicos de las Máquinas Eólicas. UNS-Energía Eólica. (2003).

[5] Alejandro Blanco Medina. Acústica Submarina para los Generadores de Energía eléctrica a partir de las Energías Renovables Marinas. Trabajo de Estudios Avanzados. E.T.S.I. Navales. UPM. (2010).

[6] M. Ragheb. Control of Wind Turbines. Available at: https://netfiles.uiuc.edu/mragheb/www/NPRE\%20475\%20Wind\%20Power\%20Systems/ Control\%20 of\%20Wind\%20Turbines. Pdf. [on-line]. (2008). [Consultado en junio de 2012].

[7] L.M. Milne-Thompson. Theoretical Hydrodynamics. 5a edición. Nueva York. Dover Publications Inc. (1996).

[8] Ira H. Abbot \& Albert E. Von Doenhoff. Theory of Wing Sections. Nueva York. Dover Publications Inc. (1958).

[9] Pijush K. Kundu. Ira M. Cohen. David R. Rowling. Fluid Mechanics. 5 a edición. Waltham, Massachusetts, EEUUU. (2012).

[10] Frank Brontsema, F.W. Wubs. Computing potential flows around Joukowski airfoils using FFTs. Bachelor thesis. University of Groningen. (2007).

[11] Airfoil Tools [on-line]. <http://airfoiltools.com/>. [Consultado el 8 de Julio de 2015].

[12] Gonzalo Pérez Gómez. Juan González-Adalid. Aplicación de la Nueva teoría de la Impulsión para el Diseño de Turbinas Axiales y Generadores Eólicos. Revista Ingeniería Naval. Páginas 117-120. Diciembre. (2003).

[13] Junior Senat. Numerical Simulation and Prediction of Loads in Marine Current Turbine Full-Scale Motor Blades. Thesis (PhD). Florida Atlantic University. (2011).

[14] Grant Ingram. Wind Turbine Blade Analysis using the Blade Element Momentum Method. Durham University. (2011). 
[15] P. L. Fraenkel. Power from marine currents. Proc. IMechE Vol. 216 Part A: J. Power and Energy. UK. (2002).

[16] D.P. Coiro, U. Maisto, F. Scherillo, S. Melone, F. Grasso. Horizontal Axis Tidal Current Turbine: Numerical and Experimental Investigations. Owemes, 20-22 April. Civitavecchia, Italia. (2006).

[17] CHRIS GARRETT AND PATRICK CUMMINS. The Efficiency of a Turbine in a Tidal Channel. J. Fluid Mech. (2007), vol. 588, pp. 243-251. Cambridge University Press. (2007).

[18] Luksa Luznik, Karen A. Flack, Ethan E. Lust, Katharin Taylor. The Effect of Surface Waves on the Performance Characteristics of a Model Tidal Turbine. Renewable Energy 58. 108-114. (2013).

[19] J. H. Lee, D. H. Kim and S. H. Rhee. Horizontal Axis Tidal Stream Turbine Analysis Using Numerical Simulation. 49th AIAA Aerospace Sciences Meeting including the New Horizons Forum and Aerospace Exposition 4 - 7 January. Orlando. Florida. (2011).

[20] Bureau Veritas. Current and Tidal Turbines. (2015).

[21] D. Wang, M. Atlar, and R. Sampson. An Experimental Investigation on Cavitation, Noise and Slipstream Characteristics of an Ocean Stream Turbines. Proc. IMechE Vol. 221 Part A: J. Power and Energy. (2006).

[22] Eckhard Praefke. The Marine Propeller Design Spiral. Second International Symposium on Marine Propulsors. June. Hamburg. Germany. (2011).

[23] David Hernán Menéndez Arán. Hydrodynamic Optimization and Design of Marine Current Turbines and Propellers. Thesis (PhD). University of Texas. (2013).

[24] David Hartwanger and Dr Andrej Horvat. 3D Modelling of a Wind Turbine Using CFD. NAFEMS Conference. United Kingdom. (2008).

[25] Sufian Fuad Sufian and Ming Li. 3D-CFD Numerical Modeling of Impacts From Horizontal Axis Tidal Turbines in The Near Region. University of Liverpool. (2013).

[26] Aleksandar Bengin. Three-Dimensional Rotor Flow Calculation. FME Transactions 33, 33-39. University of Belgrade. (2005).

[27] Simon Gant and Tim Stallard. Modelling a Tidal Turbine in Unsteady Flow. Paper No. ISOPE-2008, University of Manchester. (2008).

[28] P. M. Singh and Y. D. Choi. Shape Design and CFD Analysis on a 1MW-class Horizontal Axis Tidal Current Turbine Blade. 6th International Conference on Pumps and Fans with Compressors and Wind Turbines. (2013). 
[29] Theodor Popescu. A Foundation in Distributions of Glauert's Theory. $3^{\text {rd }}$ Workshop on Vortex Dominated Flows. Timisoara. Romania. June 1 - 2. (2007).

[30] Gareth I. Gretton, David M. Ingram, lan G. Bryden. Results From Blade Element Momentum and RANS Analyses of a Practical Full-scale Horizontal Axis Tidal Current Turbine. University of Edinburgh. (2013).

[31] W. M. J. Batten, M. E. Harrison, A. S. Bahaj. Accuracy of the Actuator Disc-RANS Approach for Predicting the Performance and Wake of Tidal Turbines. Royal Society Publishing. UK. (2013).

[32] Cécile Münch, Marcel Vonlanthen, Joao Gomes, Romain Luquet, Paul Guinard, François Avellan. Design and Performance Assesment of a Tidal Ducted Turbine. 3rd IAHR International Meeting of the Workgroup on Cavitation and Dynamic Problems in Hydraulic Machinery and Systems. Brno. Czech Republic. October 14-16. (2009).

[33] C. Lartiga and C. Crawford. Actuator Disk Modeling in Support of Tidal Turbine Rotor Testing. 3rd International Conference on Ocean Energy. 6 October. Bilbao. (2010).

[34] A.S. Bahaj, L.E. Myers, M.D. Thomson \& N. Jorge. Characterising the Wake of Horizontal Axis Marine Current Turbines. Proceedings of the $7^{\text {th }}$ European Wave and Tidal Energy. Porto. Portugal. (2009).

[35] Duncan M. McNae, J. Michael R. Graham. Computational Modelling of Unsteady Rotor Effects. Oxford Tidal Energy Workshop. 29-30 March. (2012).

[36] X.BAI, E.J. AVITAL and J. J. R. WILLIAMS. Numerical Simulation of a Marine Current Turbine in Turbulent Flow. Recent Researches in Environment and Biomedicine. ISBN: 9781-61804-075-6. Recent Researches in Environment and Biomedicine. University of London. [SIN FECHA]

[37] M.J. Churchfield, Y. Li, and P.J. Moriarty. A Large-Eddy Simulation Study of Wake Propagation and Power Production in an Array of Tidal-Current Turbines. To be presented at the 9th European Wave and Tidal Energy Conference 2011. Southhampton, England Conference Paper. NREL/CP-5000-51765. (2011).

[38] Ian Masters, Rami Malki*, Alison Williams and Nick Croft. The Influence of Turbulence Model on Wake Structure of TSTs when used with a Coupled BEM-CFD Model. Oxford Tidal Energy Workshop. 29-30 March. (2012).

[39] Dr. Ganesh Rajagopalan. Incompressible Flow Over Airfoils. Iowa State University. (2005).

[40] Nitin Kolekar, Zhen Hu, Arindam Banerjee, Xiaoping Du. Hydrodynamic Design and Optimization of Hydro-kinetic Turbines Using a Robust Design Method. Proceedings of the 1st Marine Energy Technology Symposium. METS13. April 10-11, Washington, D.C. (2013). 
[41] Brenden Epps, Julie Chalfant, Richard Kimball, Alexandra Techet, Kevin Flood, and Chrysssostomos Chryssostomidis. OpenProp: An Open-source Parametric Design and Analysis Tool for Propellers. [SIN FECHA DE PUBLICACIÓN DISPONIBLE].

[42] Samuel Harding, Jim Thomsony, Brian Polagyey, Marshall Richmondz, Vibhav Durgeshz and Ian Bryden. Extreme Value Analysis of Tidal Stream Velocity Perturbations. University of Edinburgh. (2013).

[43]Suchi Subhra Mukherji. Design and critical performance evaluation of horizontal axis hydrokinetic turbines. Thesis (PhD). Missouri University of Science and Technology. (2010).

[44] D. Hart, and D. Whale. A Review of Cavitation-Erosion Resistant Weld Surfacing Alloys for Hydroturbines. [SIN FECHA DE PUBLICACIÓN DISPONIBLE].

[45] Carlton Byrne, William Cherrett, Roger Grosvenor, Allan Mason-Jones, Ceri Morris, Daphne O'Doherty, Tim O'Doherty and Paul Prickett. An Investigation into the Possible Effects of Cavitation on a Horizontal Axis Tidal Turbine. Cardiff University. (2013).

[46] W.M.J. Batten, A.S. Bahaj, A.F. Molland, J.R. Chaplin. Hydrodynamics of Marine Current Turbines. Renewable Energy 31. 249-256. (2006).

[47] Chul-Hee Jo, Jun-Ho Lee, Do-Youb Kim, and Kang-Hee Lee. Performance of Horizontal Axis Tidal Current Turbine with Blade Deformation. Inha University. (2013).

[48] G.S. Bir, M.J. Lawson, and Y. Li. Structural Design of a Horizontal-Axis Tidal Current Turbine Composite Blade. Presented at the ASME 30th International Conference on Ocean, Offshore, and Arctic Engineering Rotterdam. The Netherlands. (2011).

[49] R.E. Murray, K. Gracie, D.A. Doman, M.J. Pegg and C.M. Johnstone. Design of a Passively Adaptive Rotor Blade for Optimized Performance of a Horizontal-Axis Tidal Turbine. Dalhousie University. (2013).

[50] R.F. Nicholls-Lee, S.R. Turnock, S.W. Boyd. A Method for Analysing Fluid Structure Interactions on a Horizontal Axis Tidal Turbine. University of Southampton. (2013).

[51] J.E. Perez Ipiña, A.A. Yawny, A. Buschizazzo, E. Mikkelson. Comportamiento Mecánico de Materiales Compuestos para Aplicaciones en Palas de Aerogeneradores de Potencia. 170 CBECIMat - Congresso Brasileiro de Engenharia e Ciência dos Materiais. 15 a 19 de Noviembre. (2006).

[52] Peter Davies, Grégory Germain, Benoit Gaurier, Amélie Boisseau, Dominique Perreux. Evaluation of the durability of composite tidal turbine blades. France. (2013).

[53] Warren C.Young. Richard G. Budynas. Roark's Formulas for Stress and Strain. 7ạ Edición. Singapore. MacGraw-Hill. (2002).

[54] N. S. Asseff. Design and Finite Element Analysis of an Ocean Current Turbine Blade. Thesis (PhD). Florida Atlantic University. (2009).

[55] R.F. Nicholls-Lee, S.R. Turnock, S.W. Boyd. Application of bend-twist coupled blades for horizontal axis tidal turbines. Renewable Energy 50. 541-550. (2013). 
[56] Marie Lunde Sæterstad. Dimensioning Loads for a Tidal Turbine. Thesis (PhD). Norwegian University of Science and Technology Department of Energy and Process Engineering. (2011).

[57] Richard Evans, Ross McAdam, Marcus Royle, Luke McEwen. Optimum Geometry for Axial Flow Free Stream Tidal Turbine Blades. UK. (2013).

[58] Apuntes científicos [on-line]. <http://apuntescientificos.org/>. [Consultado el 6 de junio de 2015].

[59] Myra L. Blaylock, David C. Maniaci, Brian R. Resor. Numerical Simulations of Subscale Wind Turbine Rotor Inboard Airfoils at Low Reynolds Number. Sandia National Laboratories. EEUU. (2015).

[60] Amable López Piñeiro, Andrés Somolinos Sánchez, Luis Ramón Núñez Rivas, Miguel Santamaría Romojaro. Modelado y Simulación de Dispositivos Fondeados para el Aprovechamiento de la Energía de las Corrientes Marinas. IV jornadas de automática marítima. Octubre 21-22. Centro Tecnológico Naval y del Mar (Murcia). (2010).

[61] Luis Ramón Núñez Rivas, Amable López Piñeiro, Eva Novoa Rojas, Alfonso Carneros Lozano. El Generador para aprovechamiento energético de corrientes marinas GESMEY. Revista Ingeniería Naval. Junio. 84-91. (2011).

[62] Luis R. N. Rivas, Amable L. Piñeiro, Jose A. S. Sanchez, Eva M.N. Rojas, Alfonso C. Lozano. New Steps in the Development of the Second Generation TEC GESMEY. E.T.S. de Ingenieros Navales. (2010). 


\section{ANEXO I. CÁLCULO DE LAS FUERZAS DE SUSTENTACIÓN Y ARRASTRE PARA EL CASO DE UN ALA DE DIMENSIÓN FINITA}

En diversas publicaciones relacionadas con la Mecánica de Fluidos dedican parte de su estudio a calcular la fuerza de sustentación y de arrastre que se produce en un ala cuando ésta se mueve con velocidad constante $U$ en el seno de un fluido de densidad constante $\rho$.

De la observación de este fenómeno, se sabe que el fluido forma aguas abajo, dos vórtices con la misma intensidad de circulación $\Gamma$ aunque opuestos en el sentido de giro. Suponiendo que si la envergadura del ala es $2 a$, el centro de estos vórtices estarán a esta distancia también.

El problema se plantea del siguiente modo, se escoge una frontera paralelepipédica que contiene el ala, definida por los vértices $A B C D E F G H$. En sentido longitudinal la longitud es arbitraria y en sentido vertical y transversal, la dimensión de las aristas es $2 a$.

Estas disposiciones de reflejan esquemáticamente en la Figura 1. Y en la cara EFGH el fluido tendrá un campo de velocidades que corresponde al descrito anteriormente, el aspecto de éste se puede observar en la Figura 2, y cuyas ecuaciones son las siguientes,

$$
\begin{aligned}
& v_{y}=\frac{\Gamma}{2 \pi}\left[\frac{z}{z^{2}+(a+y)^{2}}-\frac{z}{z^{2}+(a-y)^{2}}\right] \\
& v_{z}=\frac{\Gamma}{2 \pi}\left[-\frac{a+y}{z^{2}+(a+y)^{2}}-\frac{a-y}{z^{2}+(a-y)^{2}}\right]
\end{aligned}
$$

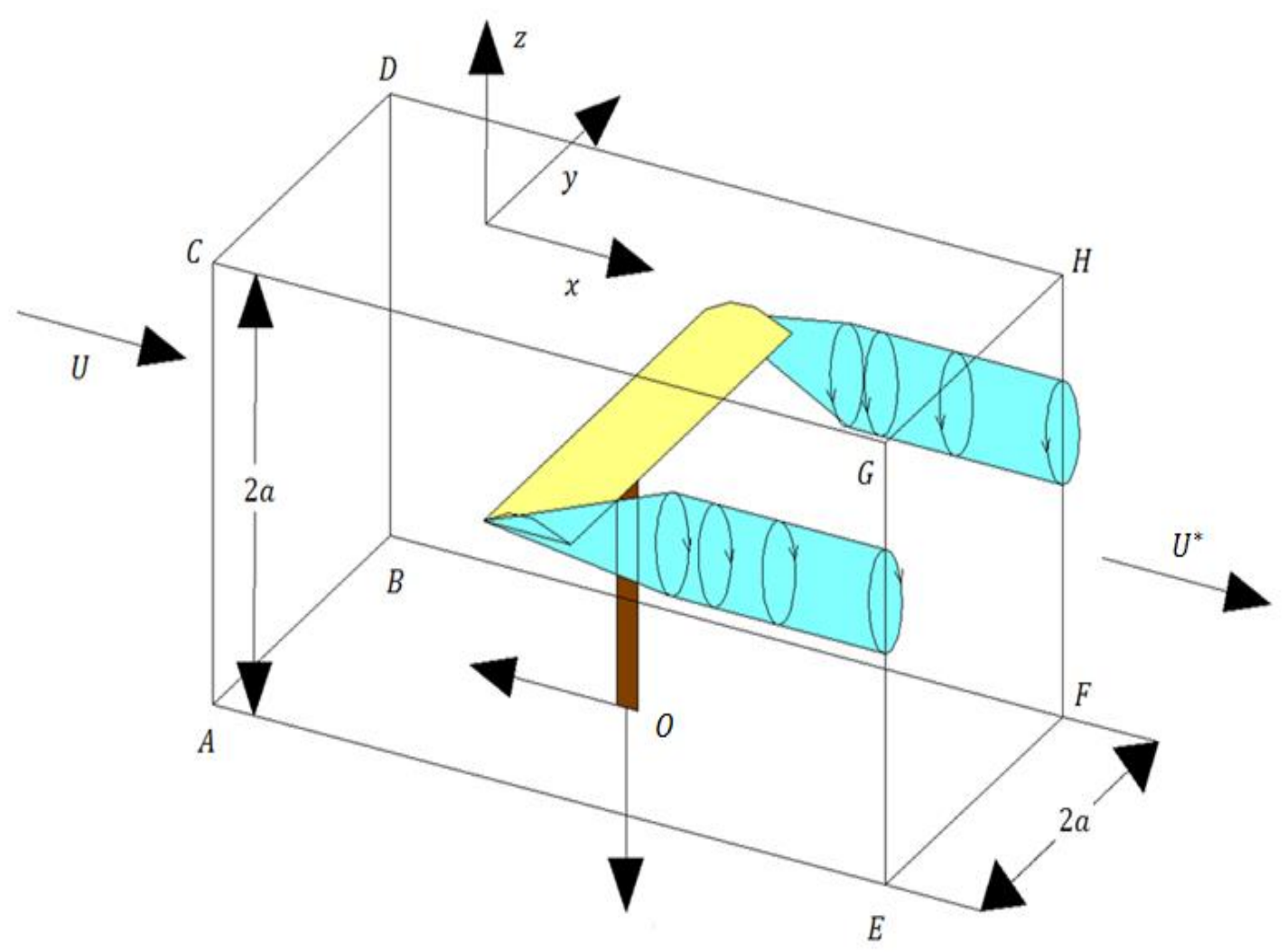

Figura 1 


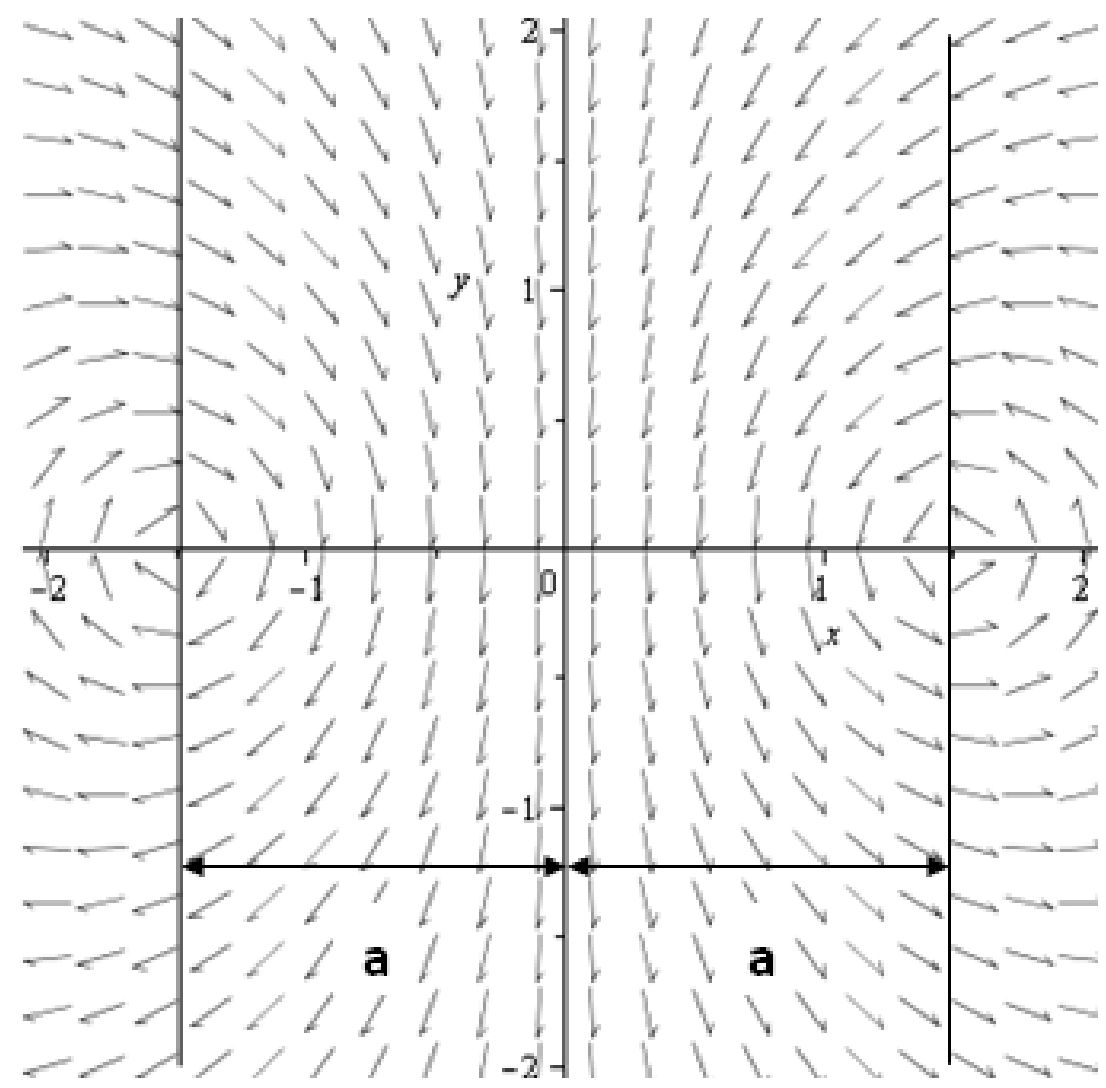

Figura 2

Con el objeto de obtener las expresiones de la fuerza de sustentación y de arrastre generada por el ala, fuerza vertical y horizontal aplicadas en el punto 0 , como se indica en la Figura 1, hay que llevar a cabo el análisis del flujo de masa, de cantidad de movimiento y de energía a través de la frontera definida.

\section{Conservación de la masa}

Por lo tanto, el primer paso es plantear las ecuaciones que rigen el balance másico, que como se trata de un sistema que está en régimen estacionario y que, como se ha dicho anteriormente el fluido tiene densidad constante $\rho$, la ecuación en forma integral queda,

$$
\int_{A B C D E F G H} \rho(\bar{v} \overline{d s})=0
$$

Siendo $\bar{v}$ el vector velocidad de la partícula fluida que se encuentra en puntos del paralelepípedo definido como frontera del sistema. El vector $\overline{d s}$ representa el elemento diferencial de superficie, su dirección es normal a ésta y dirigido hacia el exterior del sistema.

Desglosando la integral de la ecuación Ec.3 en una integral por cada una de las caras de la frontera, 


$$
\int_{A B C D} \rho(\bar{v} \overline{d s})+\int_{A B E F} \rho(\bar{v} \overline{d s})+\int_{A C E G} \rho(\bar{v} \overline{d s})+\int_{B D F H} \rho(\bar{v} \overline{d s})+\int_{C D G H} \rho(\bar{v} \overline{d s})+\int_{E F G H} \rho(\bar{v} \overline{d s})=0 \quad \text { Ec. } 4
$$

$\mathrm{Y}$ ahora obteniendo el valor de cada integral,

$$
\begin{aligned}
& \int_{A B C D} \rho(\bar{v} \overline{d s})=-\rho \int_{A B C D} U d s \\
& \int_{E F G H} \rho(\bar{v} \overline{d s})=\rho \int_{E F G H} U^{*} d s
\end{aligned}
$$

Por la geometría del sistema definido, se puede asumir que la velocidad del fluido en las caras ABEF, ACEG, BDFH y CDGH es paralela a estos planos y por lo tanto, ortogonal al vector diferencial de superficie, haciendo que el producto vectorial $\bar{v} \overline{d s}$ sea nulo en estos casos. De este modo, la ecuación Ec. 4 queda,

$$
\begin{gathered}
-\rho \int_{A B C D} U d s+\rho \int_{E F G H} U^{*} d s=-\rho \int_{-a}^{a} d z \int_{-a}^{a} U d y+\rho \int_{-a}^{a} d z \int_{-a}^{a} U^{*} d y=2 a \rho \int_{-a}^{a}\left(U^{*}-U\right) d y \\
=0
\end{gathered}
$$

\section{Momento lineal}

En siguiente paso, es plantear y resolver las ecuaciones que rigen el balance de momento lineal. De forma general la ecuación es,

$$
\int_{A B C D E F G H} \rho \bar{v}(\bar{v} \overline{d s})=\sum \overline{F_{e x t}}
$$

Es decir, que el flujo de momento lineal a través de la frontera del sistema es igual a las fuerzas que actúan exteriormente. Haciendo un desarrollo análogo al anterior, esta ecuación queda de la siguiente forma,

$$
-\rho \overline{u_{x}} \int_{A B C D} U^{2} d s+\rho \int_{E F G H}\left(U^{*} \overline{u_{x}}+v_{y} \overline{u_{y}}+v_{z} \overline{u_{z}}\right) U^{*} d s=\sum \overline{F_{e x t}}
$$

Descomponiendo cada término en componentes, y con sus correspondientes límites de integración,

$$
\begin{gathered}
\rho \overline{u_{x}} \int_{-a}^{a} d z \int_{-a}^{a}\left(U^{* 2}-U^{2}\right) d y=2 a \rho \overline{u_{x}} \int_{-a}^{a}\left(U^{* 2}-U^{2}\right) d y \\
\rho \overline{u_{y}} \int_{E F G H}\left(v_{y} U^{*}\right) d s=\rho \overline{u_{y}} \int_{-a}^{a} \int_{-a}^{a} U^{*} v_{y} d z d y
\end{gathered}
$$




$$
\rho \overline{u_{z}} \int_{E F G H}\left(v_{z} U^{*}\right) d s=\rho \overline{u_{y}} \int_{-a}^{a} \int_{-a}^{a} U^{*} v_{z} d z d y
$$

Sustituyendo $\mathrm{v}_{\mathrm{y}} \mathrm{y}_{\mathrm{z}}$ con sus expresiones correspondientes de las ecuaciones Ec.1 y Ec. 2,

$$
\begin{gathered}
\rho \overline{u_{y}} \int_{E F G H}\left(v_{y} U^{*}\right) d s=\rho \frac{\Gamma}{2 \pi} \overline{u_{y}} \int_{-a}^{a} U^{*} \int_{-a}^{a}\left[\frac{z}{z^{2}+(a+y)^{2}}-\frac{z}{z^{2}+(a-y)^{2}}\right] d z d y \\
\rho \frac{\Gamma}{2 \pi} \overline{u_{z}} \int_{E F G H}\left(v_{z} U^{*}\right) d s=\rho \frac{\Gamma}{2 \pi} \overline{u_{z}} \int_{-a}^{a} U^{*} \int_{-a}^{a}\left[-\frac{a+y}{z^{2}+(a+y)^{2}}-\frac{a-y}{z^{2}+(a-y)^{2}}\right] d z d y
\end{gathered}
$$

Atendiendo función $\mathrm{v}_{\mathrm{y}}$, se puede comprobar que si se hace $\mathrm{y}=-\mathrm{y}$, esta queda así,

$$
v_{y}(-y)=\frac{z}{z^{2}+(a-y)^{2}}-\frac{z}{z^{2}+(a+y)^{2}}=-\left[\frac{z}{z^{2}+(a+y)^{2}}-\frac{z}{z^{2}+(a-y)^{2}}\right]=-v_{y}(y)
$$

Por tanto la integral parcial en y de la ecuación Ec.13 es igual a cero ya que el intervalo de integración es simétrico con respecto a esta variable, esto implica que la componente de la fuerza en la dirección $\mathrm{F}_{\mathrm{y}}$ es nula. Para la componente vertical, la expresión es,

$\rho \frac{\Gamma}{2 \pi} \overline{u_{z}} \int_{-a}^{a} U^{*} \int_{-a}^{a}\left[-\frac{a+y}{z^{2}+(a+y)^{2}}-\frac{a-y}{z^{2}+(a-y)^{2}}\right] d z d y=-2 \rho \frac{\Gamma}{2 \pi} \overline{u_{z}} \int_{-a}^{a} U^{*}\left[\arctan \left(\frac{a}{a+y}\right)+\arctan \left(\frac{a}{a-y}\right)\right] d y$

Llamando,

$$
\varphi(y)=\arctan \left(\frac{a}{a+y}\right)+\arctan \left(\frac{a}{a-y}\right)
$$

La expresión de la componente en la dirección z queda,

$$
F_{z}=-\rho \frac{\Gamma}{\pi} \int_{-a}^{a} U^{*} \varphi(y) d y
$$

De la ecuación Ec.10 se obtiene la componente horizontal,

$$
F_{x}=2 a \rho \int_{-a}^{a}\left(U^{* 2}-U^{2}\right) d y
$$




\section{Demostración del Teorema de Kutta-Joukowski}

Si se aproxima $U^{*} \cong U$, y se considera que en la dirección z el volumen de control está limitado por los planos $z \rightarrow-\infty$ y $z \rightarrow \infty$, la fuerza ascendente en el ala es, de acuerdo a la ecuación 14,

$$
\mathrm{F}_{\mathrm{z}}=\rho \frac{\Gamma}{2 \pi} U \int_{-\infty}^{\infty} d z \int_{-a}^{a}\left[-\frac{a+y}{z^{2}+(a+y)^{2}}-\frac{a-y}{z^{2}+(a-y)^{2}}\right] d y
$$

Integrando esta expresión,

$$
\begin{gathered}
\rho \frac{\Gamma}{2 \pi} U \int_{-\infty}^{\infty} d z \int_{-a}^{a}\left[-\frac{a+y}{z^{2}+(a+y)^{2}}-\frac{a-y}{z^{2}+(a-y)^{2}}\right] d y=-\rho U\left(\frac{\Gamma}{2 \pi}\right) \int_{-\infty}^{\infty} \ln \left[1+\left(\frac{2 a}{z}\right)^{2}\right] d z= \\
=-\rho U\left(\frac{\Gamma}{2 \pi}\right)\left\{z \ln \left[1+\left(\frac{2 a}{z}\right)^{2}\right]+4 \operatorname{aarctan}\left(\frac{z}{2 a}\right)\right\}_{-\infty}^{\infty}=-\rho U\left(\frac{\Gamma}{2 \pi}\right)\left\{4 a \frac{\pi}{2}-4 a\left(-\frac{\pi}{2}\right)\right\}= \\
=-\rho U \Gamma(2 a)
\end{gathered}
$$

Por tanto, la fuerza ascendente por unidad de longitud es,

$$
L=\rho U \Gamma
$$

\section{Balance de energía.}

Y ya finalmente, solamente queda evaluar el balance de energía, para ello hay que tener en cuenta que la energía entra en el sistema a través de la fuerza $F_{x}$ que arrastra el ala a la velocidad $U$, de aquí que la ecuación que rige en este caso es,

$$
\int_{A B C D E F G H} \rho \frac{1}{2} v^{2}(\bar{v} \overline{d s})=F_{x} U
$$

Desarrollando igual que en los pasos anteriores,

$$
\begin{gathered}
-\rho \frac{1}{2} \int_{A B C D} U^{2}(U d s)+\rho \frac{1}{2} \int_{E F G H}\left(U^{* 2}+v_{y}^{2}+v_{z}^{2}\right) U^{*} d s=F_{x} U \\
\rho \frac{1}{2} \int_{-a}^{a}\left(U^{* 3}-U^{3}\right) d y \int_{-a}^{a} d z+\rho \frac{1}{2} \int_{-a}^{a} U^{*} \int_{-a}^{a}\left(v_{y}^{2}+v_{z}^{2}\right) d z d y \\
=F_{x} U
\end{gathered}
$$

$$
\rho a \int_{-a}^{a}\left(U^{* 3}-U^{3}\right) d y+\rho \frac{1}{2}\left(\frac{\Gamma}{2 \pi}\right)^{2} \int_{-a}^{a} U^{*} \int_{-a}^{a}\left(\left[\frac{z}{z^{2}+(a+y)^{2}}-\frac{z}{z^{2}+(a-y)^{2}}\right]^{2}+\left[-\frac{a+y}{z^{2}+(a+y)^{2}}-\frac{a-y}{z^{2}+(a-y)^{2}}\right]^{2}\right) d z d y=F_{x} U
$$


Llamando,

$$
\theta(y)=\int_{-a}^{a}\left(\left[\frac{z}{z^{2}+(a+y)^{2}}-\frac{z}{z^{2}+(a-y)^{2}}\right]^{2}+\left[-\frac{a+y}{z^{2}+(a+y)^{2}}-\frac{a-y}{z^{2}+(a-y)^{2}}\right]^{2}\right) d z
$$

La ecuación Ec. 23 queda,

$$
\rho a \int_{-a}^{a}\left(U^{* 3}-U^{3}\right) d y+\rho \frac{1}{2}\left(\frac{\Gamma}{2 \pi}\right)^{2} \int_{-a}^{a} U^{*} \theta(y) d y=F_{x} U
$$

Despejando la circulación,

$$
\Gamma=2 \pi \sqrt{\frac{2 F_{x} U-2 \rho a \int_{-a}^{a}\left(U^{* 3}-U^{3}\right) d y}{\rho \int_{-a}^{a} U^{*} \theta(y) d y}}
$$

\section{Aplicación de la teoría de perfiles sustentadores bidimensionales}

De acuerdo a esta teoría, cuando un perfil sustentador bidimensional se mueve en el seno de un fluido a velocidad constante, se producen en él dos fuerzas, una perpendicular a la velocidad del fluido que es la fuerza de sustentación y otra paralela que se opone al movimiento, estas fuerzas se denotarán como L y D respectivamente, y cuyas expresiones son,

$$
\begin{aligned}
L & =\frac{1}{2} \rho c c_{L} V^{2} \\
D & =\frac{1}{2} \rho c c_{D} V^{2}
\end{aligned}
$$
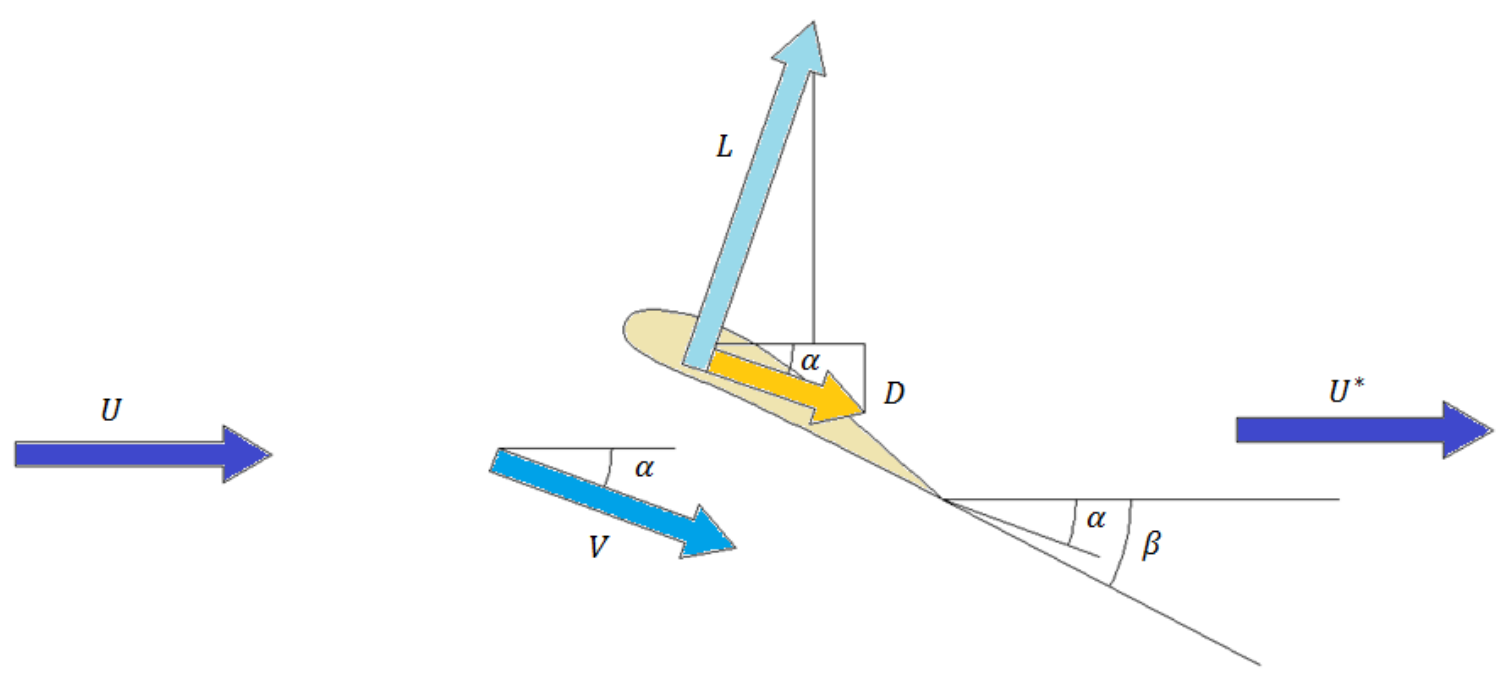

Figura 3 
Proyectando y sumando estas fuerzas en las direcciones vertical y horizontal, y asumiendo que aunque el módulo $V$ de la velocidad del fluido en las cercanías del perfil sustentador es una incógnita del problema planteado, se puede aproximar a $V \approx U$.

$$
\begin{aligned}
& l=\frac{1}{2} \rho c V^{2}\left[c_{L} \cos (\alpha)-c_{D} \operatorname{sen}(\alpha)\right] \approx \frac{1}{2} \rho c U^{2}\left[c_{L} \cos (\alpha)-c_{D} \operatorname{sen}(\alpha)\right] \\
& d=\frac{1}{2} \rho c V^{2}\left[c_{L} \operatorname{sen}(\alpha)+c_{D} \cos (\alpha)\right] \approx \frac{1}{2} \rho c U^{2}\left[c_{L} \operatorname{sen}(\alpha)+c_{D} \cos (\alpha)\right]
\end{aligned}
$$

Como se puede ver en la Figura 3, el ángulo de ataque real $\gamma$ que interviene en el perfil sustentador es igual a,

$$
\gamma=\beta-\alpha
$$

Siendo $\beta$ el ángulo de paso geométrico de la sección con la horizontal,

Despejando $\alpha$,

$$
\alpha=\beta-\gamma
$$

Identificando las expresiones anteriores con las componentes $\mathrm{F}_{\mathrm{x}}$ y $\mathrm{F}_{\mathrm{Z}}$ obtenidas en las ecuaciones $\mathrm{Ec}$. 18 y E. 19,

$$
\begin{gathered}
F_{z}=\int_{-a}^{a} l d y=\frac{1}{2} \rho U^{2} \int_{-a}^{a} c\left[c_{L} \cos (\alpha)-c_{D} \operatorname{sen}(\alpha)\right] d y=\rho \frac{\Gamma}{\pi} \int_{-a}^{a} U^{*} \varphi(y) d y \\
F_{x}=\int_{-a}^{a} d d y=\frac{1}{2} \rho U^{2} \int_{-a}^{a} c\left[c_{L} \operatorname{sen}(\alpha)+c_{D} \cos (\alpha)\right] d y=2 a \rho \int_{-a}^{a}\left(U^{* 2}-U^{2}\right) d y
\end{gathered}
$$

Operando,

$$
\begin{gathered}
\int_{-a}^{a}\left\{\frac{1}{2} U^{2} c\left[c_{L} \cos (\alpha)-c_{D} \operatorname{sen}(\alpha)\right]-\frac{\Gamma}{2 \pi} U^{*} \varphi(y)\right\} d y=0 \\
\int_{-a}^{a}\left\{\frac{1}{2} U^{2} c\left[c_{L} \operatorname{sen}(\alpha)+c_{D} \cos (\alpha)\right]-2 a\left(U^{* 2}-U^{2}\right)\right\} d y=0
\end{gathered}
$$

De estas ecuaciones se puede deducir que,

$$
\begin{aligned}
& \frac{1}{2} \rho U^{2} c\left[c_{L} \cos (\alpha)-c_{D} \operatorname{sen}(\alpha)\right]-2 \rho \frac{\Gamma}{2 \pi} U^{*} \varphi(y)=0 \\
& \frac{1}{2} \rho U^{2} c\left[c_{L} \operatorname{sen}(\alpha)+c_{D} \cos (\alpha)\right]-2 a \rho\left(U^{* 2}-U^{2}\right)=0
\end{aligned}
$$

Despejando $U^{*}$ de esta última, y sustituyendo la ecuación Ec. 32, 


$$
U^{*}=U \sqrt{\frac{c}{4 a}\left[c_{L} \operatorname{sen}(\beta-\gamma)+c_{D} \cos (\beta-\gamma)\right]+1}
$$

Hay que tener en cuenta que en la ecuación anterior el factor $c_{D} \cos (\beta-\gamma)$ ha de ser negativo en algunas secciones de ala para que se pueda cumplir con la ecuación de continuidad.

\section{Resolución de las ecuaciones.}

En este momento ya se han deducido todas las ecuaciones que intervienen en el problema, y las incógnitas a resolver son la circulación $\Gamma$, la distribución de velocidades $\mathrm{U}^{*}$ y los ángulos de ataque $\gamma$ , partiendo de una geometría del ala definida por la envergadura $2 \mathrm{a}$, distribución de cuerdas c , la distribución de ángulos de paso geométrico $\beta$ y el perfil sustentador de cada sección transversal.

La obtención de soluciones exactas resulta prácticamente imposible, ya que intervienen expresiones integrales que junto con el manejo de valores tabulados como son los coeficientes de sustentación y arrastre. Así que resulta adecuada la aplicación de un esquema numérico con el que se puedan obtener soluciones aproximadas. Para definir este esquema, se divide el intervalo [ $-\mathrm{a}$, a] en $\mathrm{n}$ partes que se identificarán con el subíndice $\mathrm{k}$, la longitud de cada una de estas partes es $\delta y_{k}$.

La forma de proceder es primeramente suponer una distribución de ángulos de ataque $\gamma_{\mathrm{k}}$ igual a un valor constante por ejemplo, luego obtener $\mathrm{U}_{\mathrm{k}}{ }_{\mathrm{k}}$ con la expresión siguiente derivada de la ecuación Ec. 39,

$$
U_{k}^{*}=U \sqrt{\frac{c_{k}}{4 a}\left[c_{L_{k}} \operatorname{sen}\left(\beta_{k}-\gamma_{k}\right)+c_{D_{k}} \cos \left(\beta_{k}-\gamma_{k}\right)\right]+1}
$$

Con esta distribución, hay que comprobar que se cumple la ecuación de continuidad, que se obtiene discretizando la ecuación Ec.7,

$$
\sum_{k=1}^{n}\left(U_{k}^{*}-U\right) \delta y_{k} \cong 0
$$

Si se cumple la ecuación de continuidad, se calcula la circulación $\Gamma$ con la expresión,

$$
\Gamma=2 \pi \sqrt{\frac{4 U a \sum_{k=1}^{n}\left(U^{*}{ }_{k}{ }^{2}-U^{2}\right) \delta y_{k}-2 a \sum_{k=1}^{n}\left(U^{*}{ }_{k}{ }^{3}-U^{3}\right) \delta y_{k}}{\sum_{k=1}^{n} U^{*}{ }_{k} \theta\left(y_{k}\right) \delta y_{k}}}
$$

Para la que previamente hay que obtener la variable auxiliar $\theta\left(y_{k}\right)$,

$$
\theta\left(y_{k}\right)=\int_{-a}^{a}\left(\left[\frac{z}{z^{2}+\left(a+y_{k}\right)^{2}}-\frac{z}{z^{2}+\left(a-y_{k}\right)^{2}}\right]^{2}+\left[-\frac{a+y_{k}}{z^{2}+\left(a+y_{k}\right)^{2}}-\frac{a-y_{k}}{z^{2}+\left(a-y_{k}\right)^{2}}\right]^{2}\right) d z
$$


Con todo esto, el paso final es comprobar que se cumple la relación de la ecuación Ec. 37. Si se define la función,

$$
\psi_{k}=\frac{1}{2} \rho U^{2} c_{k}\left[c_{L_{k}} \cos \left(\beta_{k}-\gamma_{k}\right)-c_{D_{k}} \operatorname{sen}\left(\beta_{k}-\gamma_{k}\right)\right]-\rho \frac{\Gamma}{\pi} U^{*}{ }_{k} \varphi\left(y_{k}\right)
$$

El cumplimiento de esta relación implica que,

$$
\sum_{k=1}^{N} \psi_{k} \approx 0
$$

Siendo,

$$
\varphi\left(y_{k}\right)=\arctan \left(\frac{a}{a+y_{k}}\right)+\arctan \left(\frac{a}{a-y_{k}}\right)
$$

Las fuerzas verticales de sustentación en cada sección tienen la expresión,

$$
l_{k}=\frac{1}{2} \rho c_{k} U^{2}\left[c_{L_{k}} \cos \left(\beta_{k}-\gamma_{k}\right)-c_{D_{k}} \operatorname{sen}\left(\beta_{k}-\gamma_{k}\right)\right] \delta y_{k}
$$

Y las de arrastre,

$$
d_{k}=\frac{1}{2} \rho c_{k} U^{2}\left[c_{L_{k}} \operatorname{sen}\left(\beta_{k}-\gamma_{k}\right)+c_{D_{k}} \cos \left(\beta_{k}-\gamma_{k}\right)\right] \delta y_{k}
$$


ANEXO II. FIGURAS DEL APARTADO 7.5.1 PARA EL ESTUDIO DE LA TORSIÓN DE LAS SECCIONES DE PALA

SECCIÓN No 1. $r_{1}=1,225 m ; \beta_{1}=36,90^{\circ} ; c_{1}=0,950 m ; N A C A 63-212$

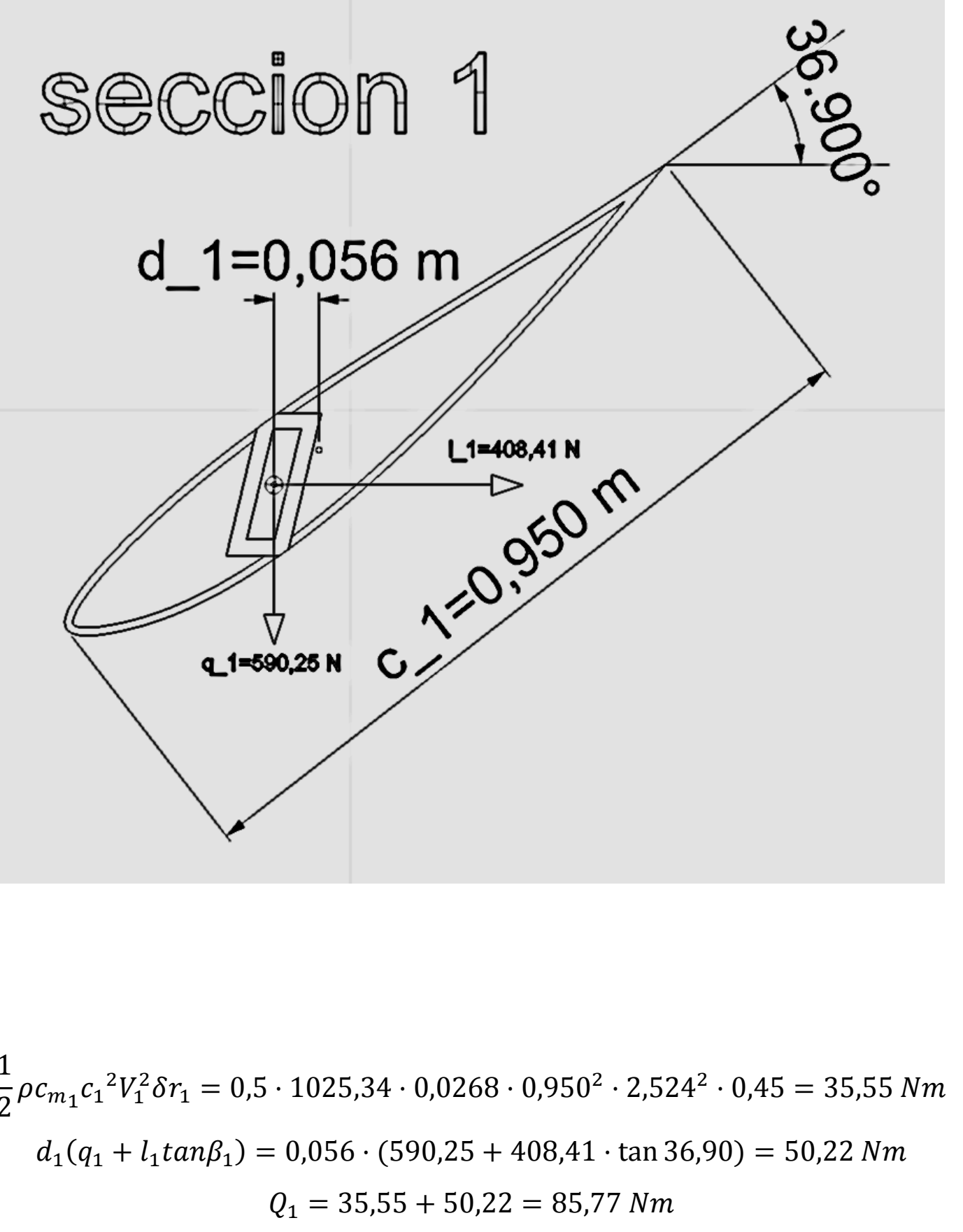


SECCIÓN No 2. $r_{2}=1,675 m ; \beta_{2}=42,90^{\circ} ; c_{2}=1,150 m ; N A C A 63-212$

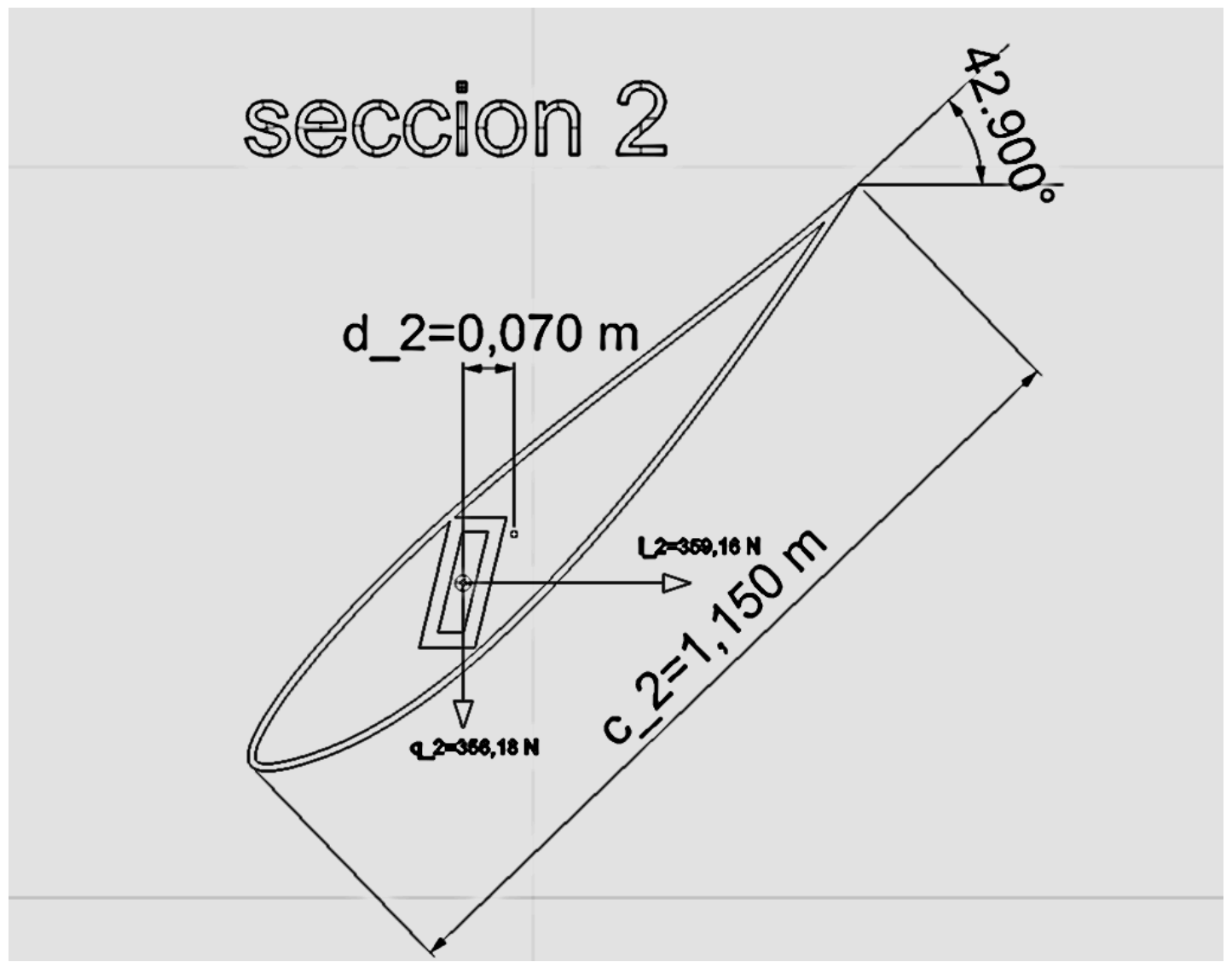

$$
\begin{gathered}
\frac{1}{2} \rho c_{m_{2}} c_{2}{ }^{2} V_{2}^{2} \delta r_{2}=0,5 \cdot 1025,34 \cdot 0,0369 \cdot 1,150^{2} \cdot 2,903^{2} \cdot 0,45=94,88 \mathrm{Nm} \\
d_{2}\left(q_{2}+l_{2} \tan \beta_{2}\right)=0,070 \cdot(356,18+359,16 \cdot \tan 42,90)=48,29 \mathrm{Nm} \\
Q_{2}=94,88+48,29=143,17 \mathrm{Nm}
\end{gathered}
$$


SECCIÓN No 3. $r_{3}=2,125 m ; \beta_{3}=48,90^{\circ} ; c_{3}=1,550 m ; N A C A 63-212$

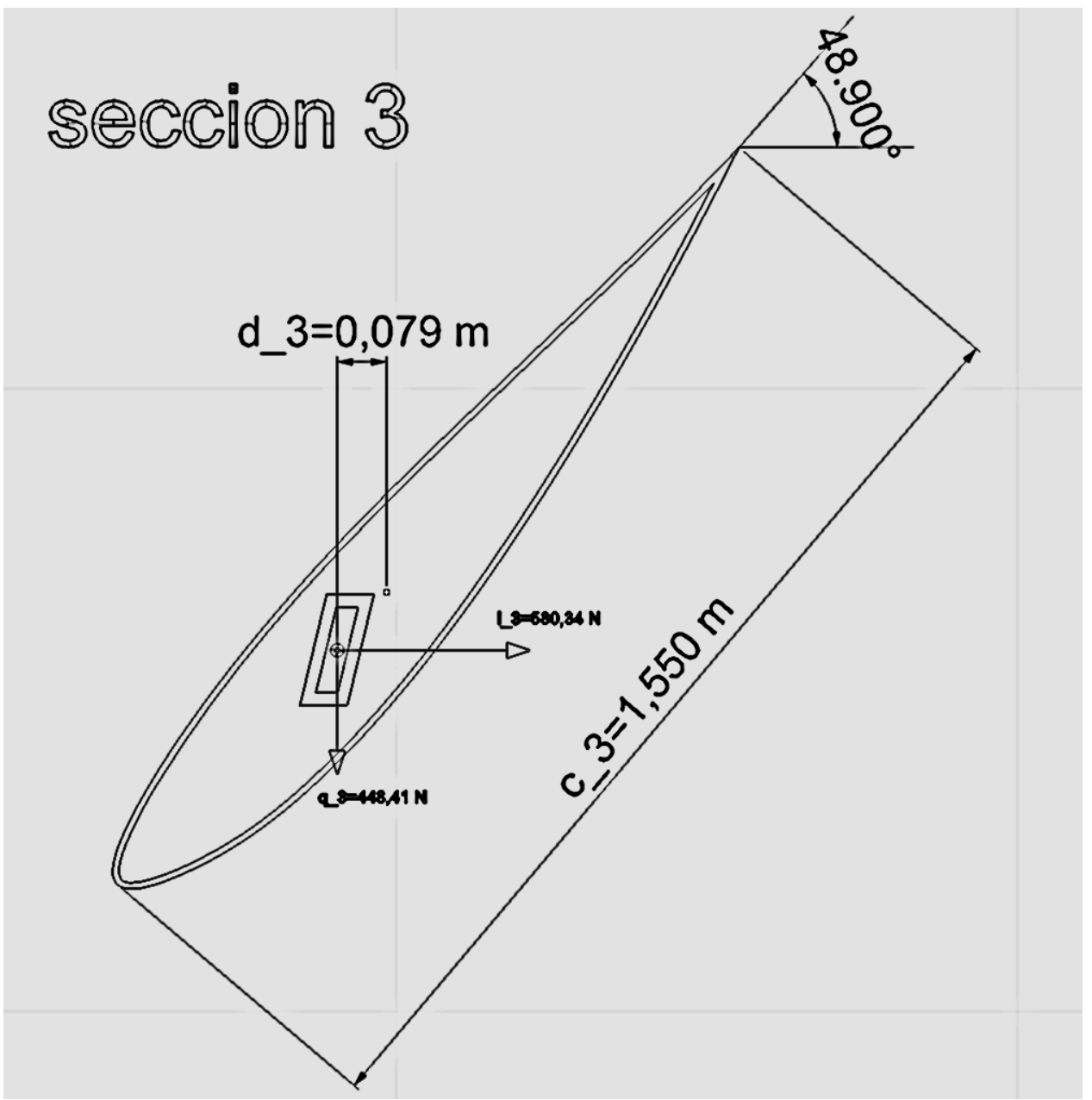

$$
\begin{gathered}
\frac{1}{2} \rho c_{m_{3}} c_{3}{ }^{2} V_{3}^{2} \delta r_{3}=0,5 \cdot 1025,34 \cdot 0,0378 \cdot 1,550^{2} \cdot 3,336^{2} \cdot 0,45=233,16 \mathrm{Nm} \\
d_{3}\left(q_{3}+l_{3} \tan \beta_{3}\right)=0,079 \cdot(580,34+448,41 \cdot \tan 48,90)=86,45 \mathrm{Nm} \\
Q_{3}=233,16+86,45=319,61 \mathrm{Nm}
\end{gathered}
$$


SECCIÓN No 4. $r_{4}=2,575 m ; \beta_{4}=54,90^{\circ} ; c_{4}=1,600 m ; N A C A 63-212$
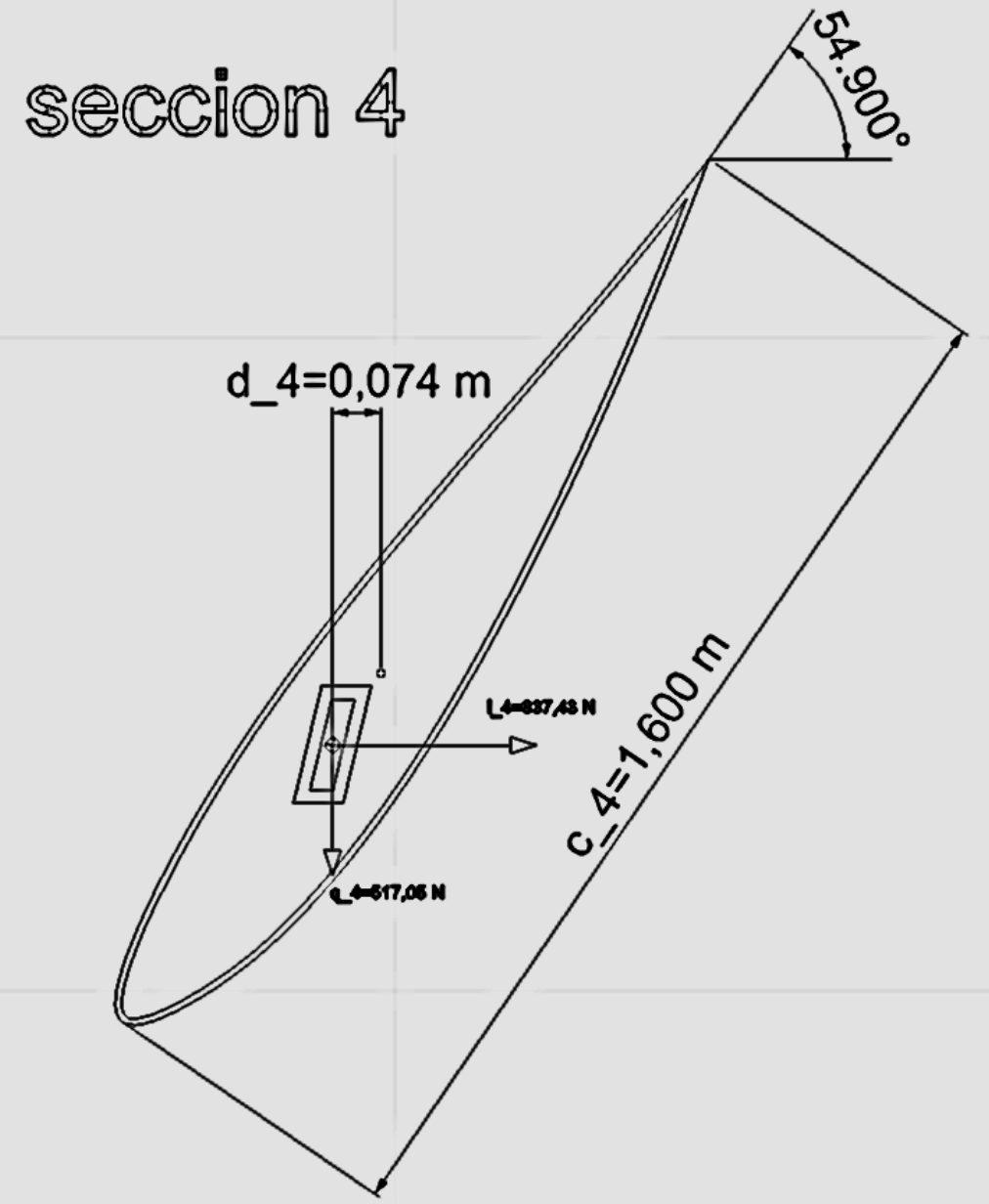

$$
\begin{gathered}
\frac{1}{2} \rho c_{m_{4}} c_{4}{ }^{2} V_{4}^{2} \delta r_{4}=0,5 \cdot 1025,34 \cdot 0,0378 \cdot 1,600^{2} \cdot 3,804^{2} \cdot 0,45=323,04 \mathrm{Nm} \\
d_{4}\left(q_{4}+l_{4} \tan \beta_{4}\right)=0,074 \cdot(517,05+837,43 \cdot \tan 54,90)=126,43 \mathrm{Nm} \\
Q_{4}=323,04+126,43=449,47 \mathrm{Nm}
\end{gathered}
$$


SECCIÓN No $5 . r_{5}=3,025 m ; \beta_{5}=60,90^{\circ} ; c_{5}=1,550 m ; N A C A 63-212$

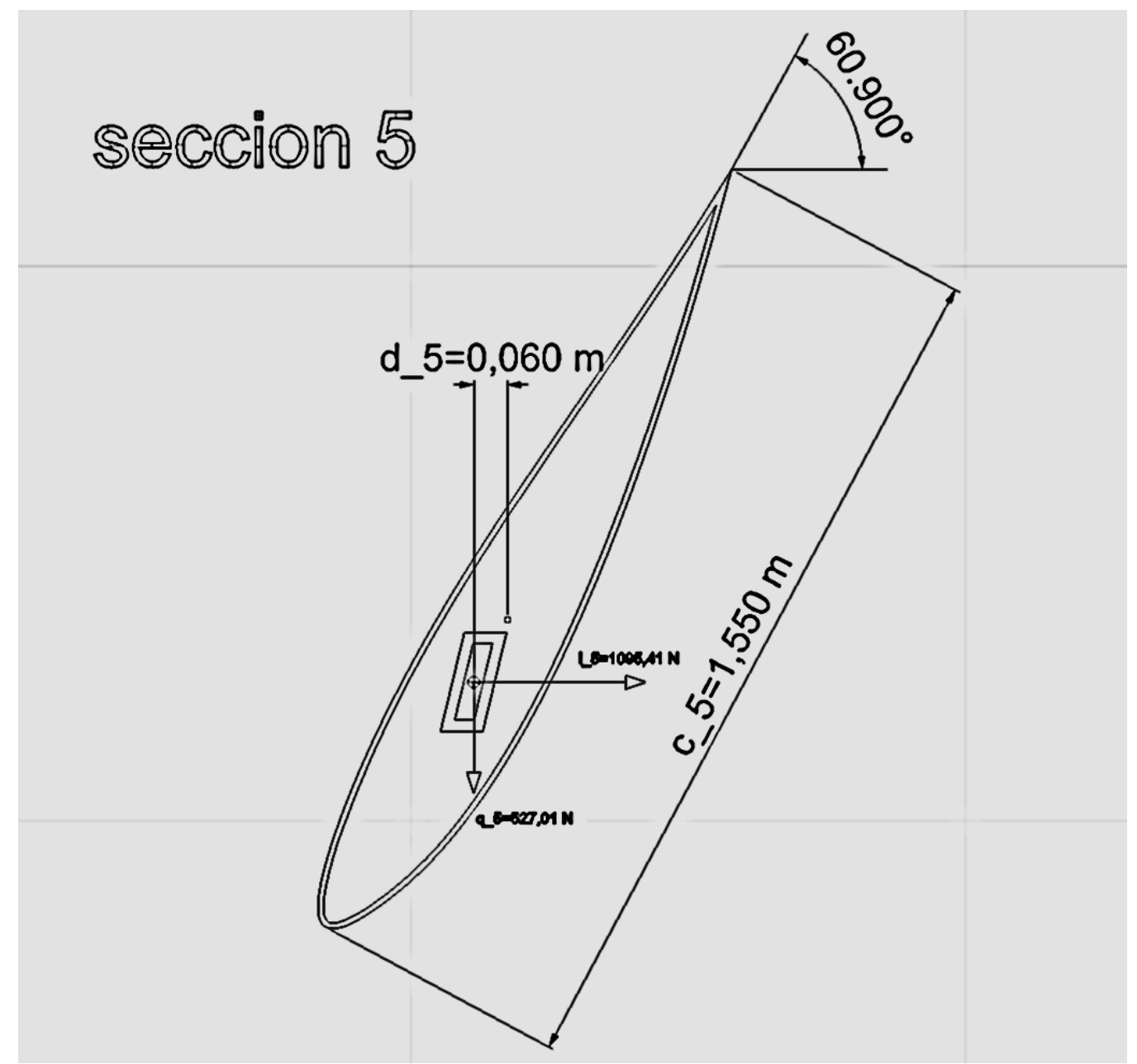

$$
\begin{gathered}
\frac{1}{2} \rho c_{m_{5}} c_{5}{ }^{2} V_{5}^{2} \delta r_{5}=0,5 \cdot 1025,34 \cdot 0,0378 \cdot 1,550^{2} \cdot 4,295^{2} \cdot 0,45=386,48 \mathrm{Nm} \\
d_{5}\left(q_{5}+l_{5} \tan \beta_{5}\right)=0,060 \cdot(527,01+1095,41 \cdot \tan 60,90)=149,704 \mathrm{Nm} \\
Q_{5}=386,48+149,704=536,18 \mathrm{Nm}
\end{gathered}
$$


SECCIÓN No 6. $r_{6}=3,475 m ; \beta_{6}=64,90^{\circ} ; c_{6}=1,500 m ; N A C A 63-212$

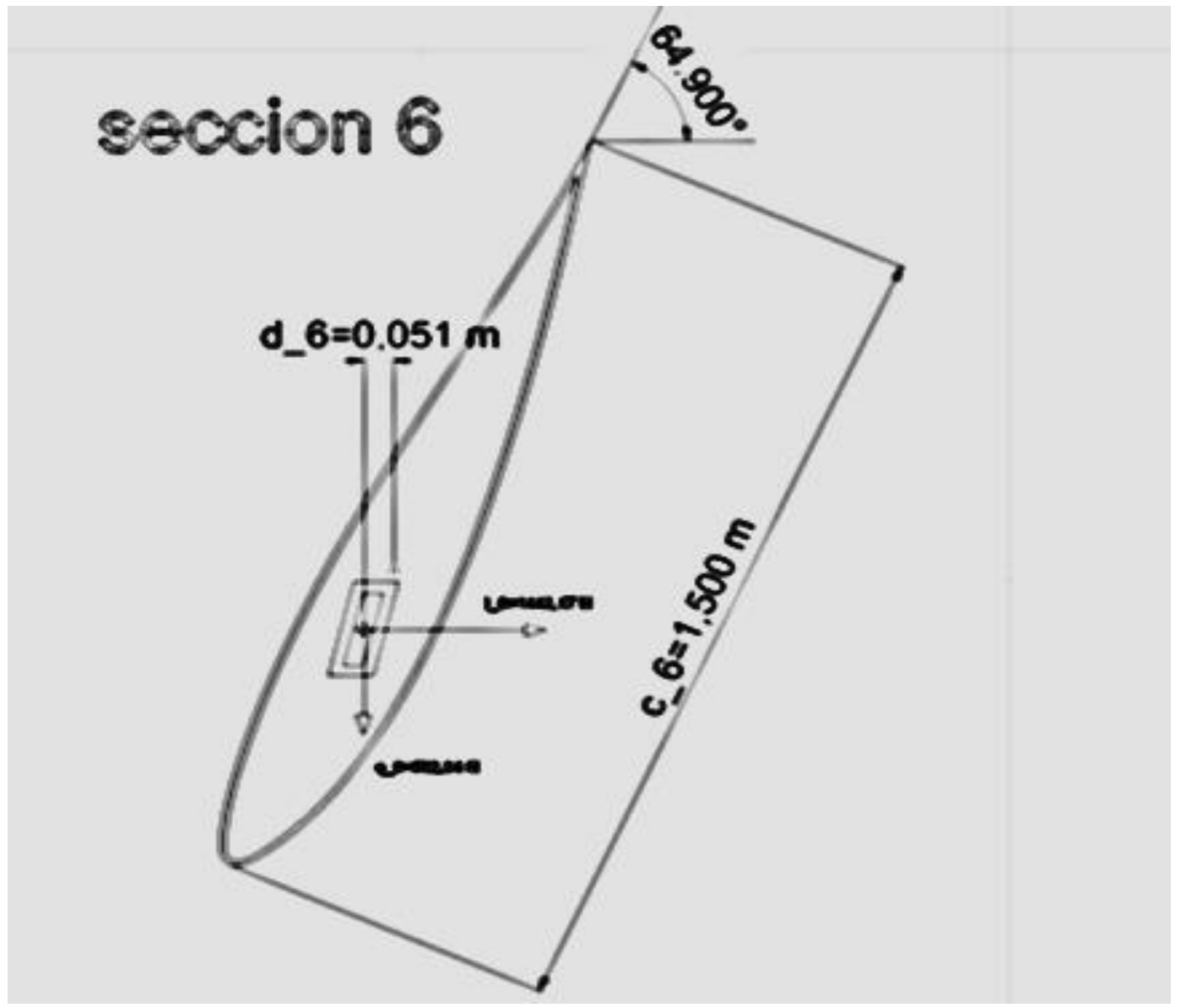

$$
\begin{gathered}
\frac{1}{2} \rho c_{m_{6}} c_{6}{ }^{2} V_{6}^{2} \delta r_{6}=0,5 \cdot 1025,34 \cdot 0,0377 \cdot 1,500^{2} \cdot 4,802^{2} \cdot 0,45=451,25 \mathrm{Nm} \\
d_{6}\left(q_{6}+l_{6} \tan \beta_{6}\right)=0,051 \cdot(582,34+1442,17 \cdot \tan 64,90)=186,71 \mathrm{Nm} \\
Q_{6}=451,25+186,71=637,96 \mathrm{Nm}
\end{gathered}
$$


SECCIÓN No 7. $r_{7}=3,925 m ; \beta_{7}=66,90^{\circ} ; c_{7}=1,450 m ; N A C A 63-212$

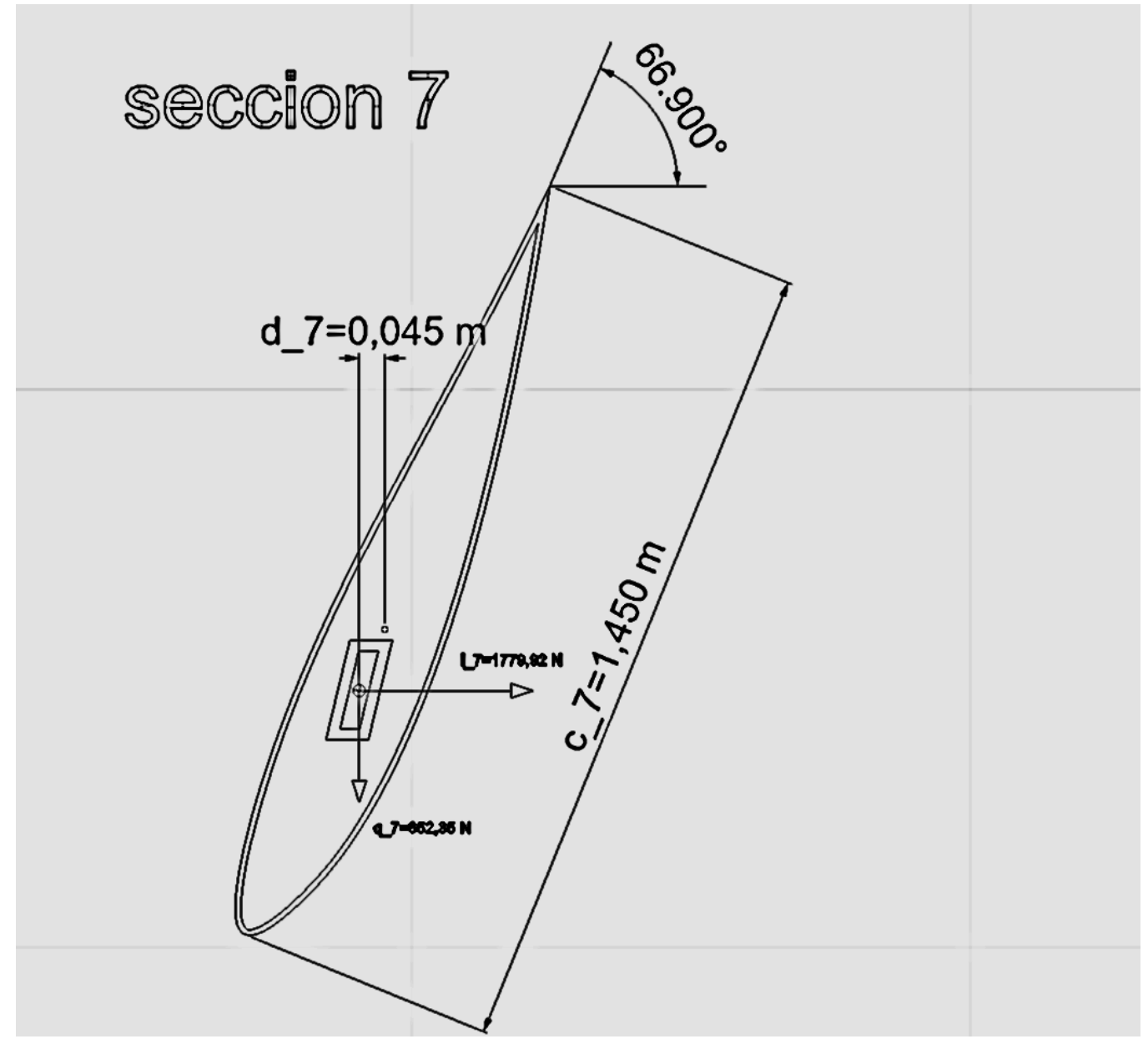

$$
\begin{gathered}
\frac{1}{2} \rho c_{m_{7}} c_{7}^{2} V_{7}^{2} \delta r_{7}=0,5 \cdot 1025,34 \cdot 0,0375 \cdot 1,450^{2} \cdot 5,322^{2} \cdot 0,45=515,19 \mathrm{Nm} \\
d_{7}\left(q_{7}+l_{7} \tan \beta_{7}\right)=0,045 \cdot(652,35+1779,92 \cdot \tan 66,90)=217,13 \mathrm{Nm} \\
Q_{7}=515,19+217,13=732,32 \mathrm{Nm}
\end{gathered}
$$


SECCIÓN No 8. $r_{8}=4,375 m ; \beta_{8}=68,90^{\circ} ; c_{8}=1,400 m ; N A C A 63-212$

\section{SECCiOR 8}

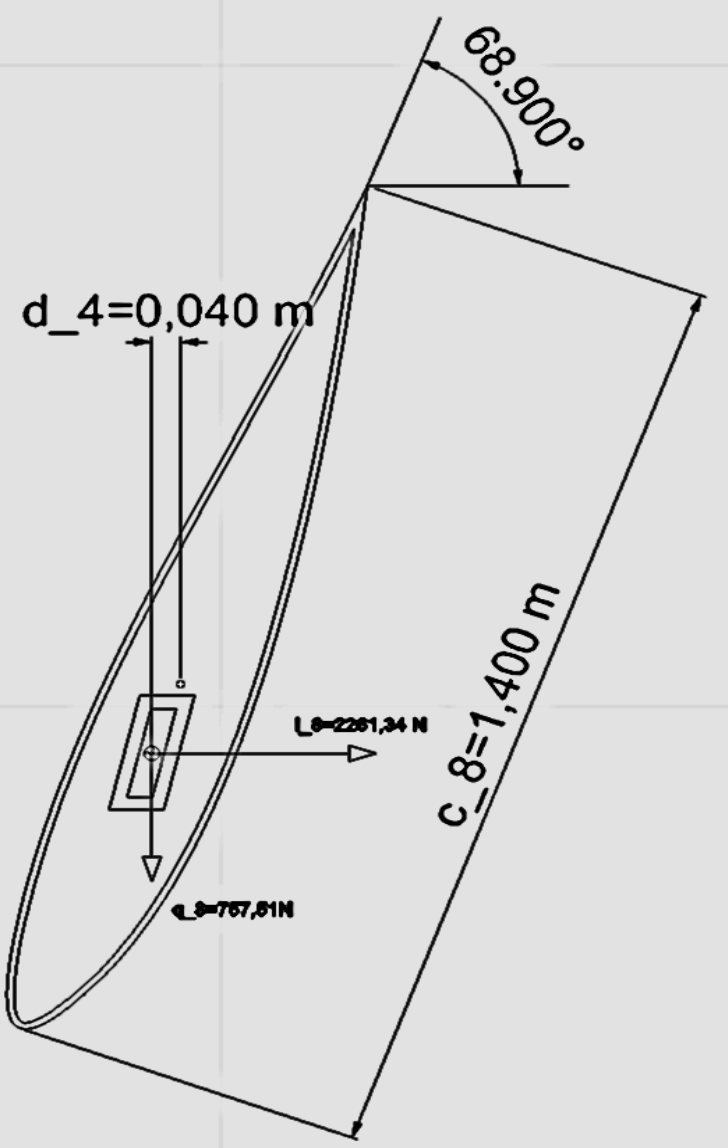

$$
\begin{gathered}
\frac{1}{2} \rho c_{m_{8}} c_{8}{ }^{2} V_{8}^{2} \delta r_{8}=0,5 \cdot 1025,34 \cdot 0,0371 \cdot 1,400^{2} \cdot 5,849^{2} \cdot 0,45=573,90 \mathrm{Nm} \\
d_{8}\left(q_{8}+l_{8} \tan \beta_{8}\right)=0,040 \cdot(757,51+2261,34 \cdot \tan 68,90)=264,72 \mathrm{Nm} \\
Q_{8}=573,90+264,72=838,62 \mathrm{Nm}
\end{gathered}
$$


SECCIÓN No 9. $r_{9}=4,825 m ; \beta_{9}=69,90^{\circ} ; c_{9}=1,350 m ; N A C A 63-212$

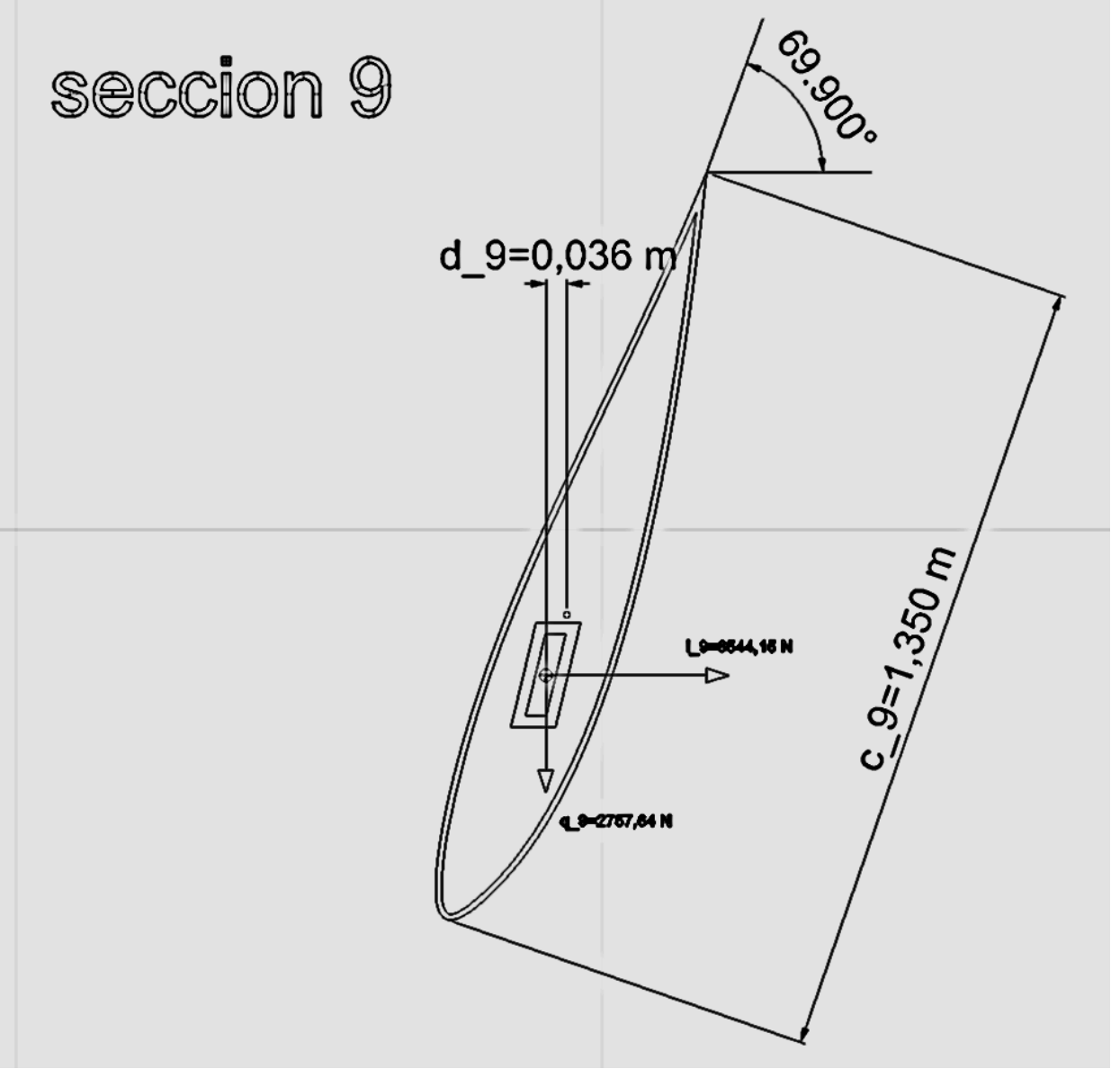

$$
\begin{gathered}
\frac{1}{2} \rho c_{m_{9}} c_{9}{ }^{2} V_{9}^{2} \delta r_{9}=0,5 \cdot 1025,34 \cdot 0,0264 \cdot 1,350^{2} \cdot 6,384^{2} \cdot 0,45=452,38 \mathrm{Nm} \\
d_{9}\left(q_{9}+l_{9} \tan \beta_{9}\right)=0,036 \cdot(2757,64+6544,15 \cdot \tan 69,90)=743,053 \mathrm{Nm} \\
Q_{9}=452,38+743,053=1195,43 \mathrm{Nm}
\end{gathered}
$$


SECCIÓN No 10. $r_{10}=5,275 m ; \beta_{10}=70,90^{\circ} ; c_{10}=1,300 m ; N A C A 63-212$

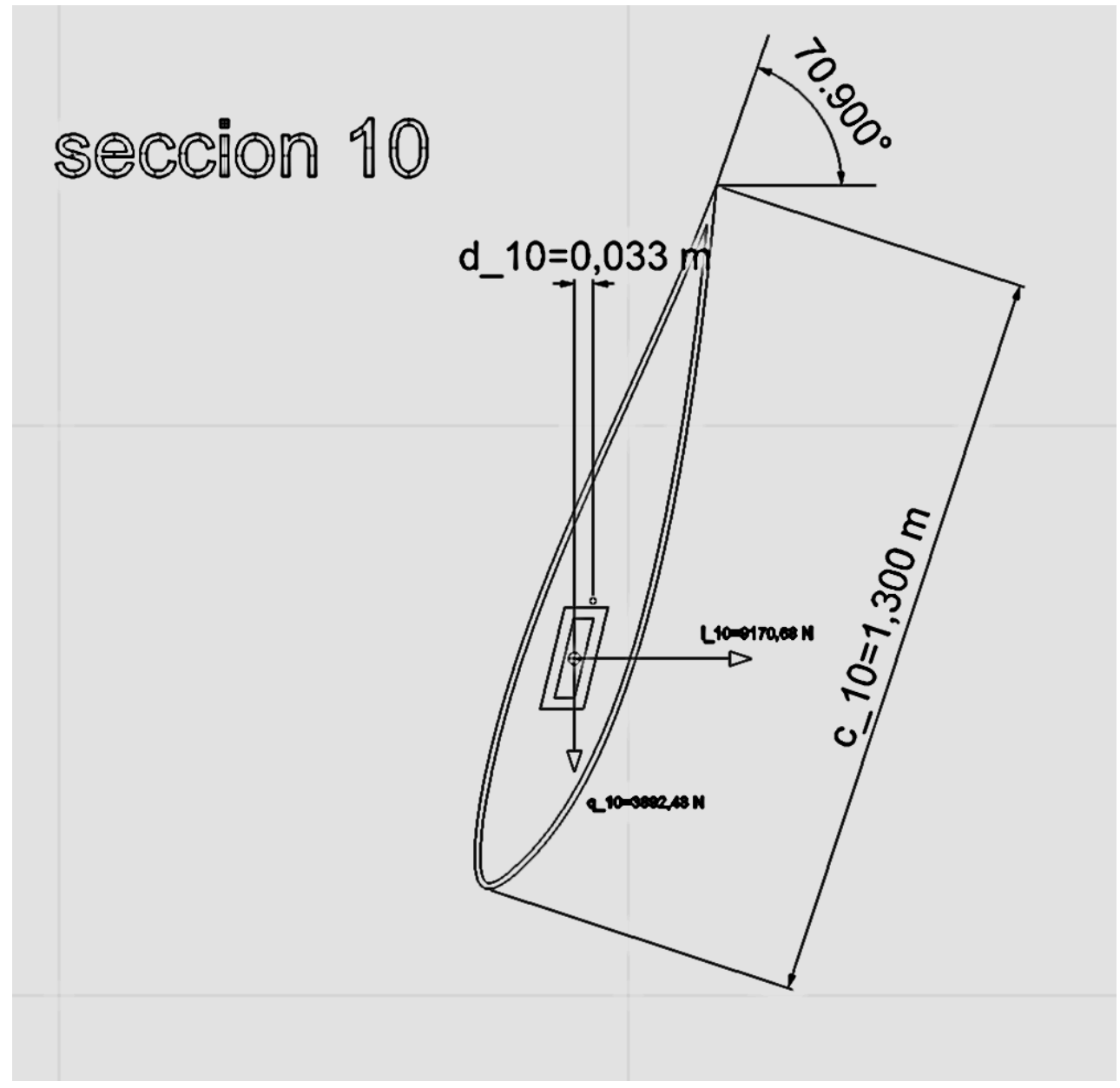

$$
\begin{aligned}
& \frac{1}{2} \rho c_{m_{10}} c_{10}{ }^{2} V_{10}^{2} \delta r_{10}=0,5 \cdot 1025,34 \cdot 0,0329 \cdot 1,300^{2} \cdot 6,923^{2} \cdot 0,45=614,79 \mathrm{Nm} \\
& d_{10}\left(q_{10}+l_{10} \tan \beta_{10}\right)=0,033 \cdot(3892,43+9170,68 \cdot \tan 70,90)=1002,40 \mathrm{Nm} \\
& Q_{10}=614,79+1002,40=1617,19 \mathrm{Nm}
\end{aligned}
$$


SECCIÓN No 11. $r_{11}=5,725 m ; \beta_{11}=71,90^{\circ} ; c_{11}=1,250 m ; N A C A 63-212$

\section{seccion 11}

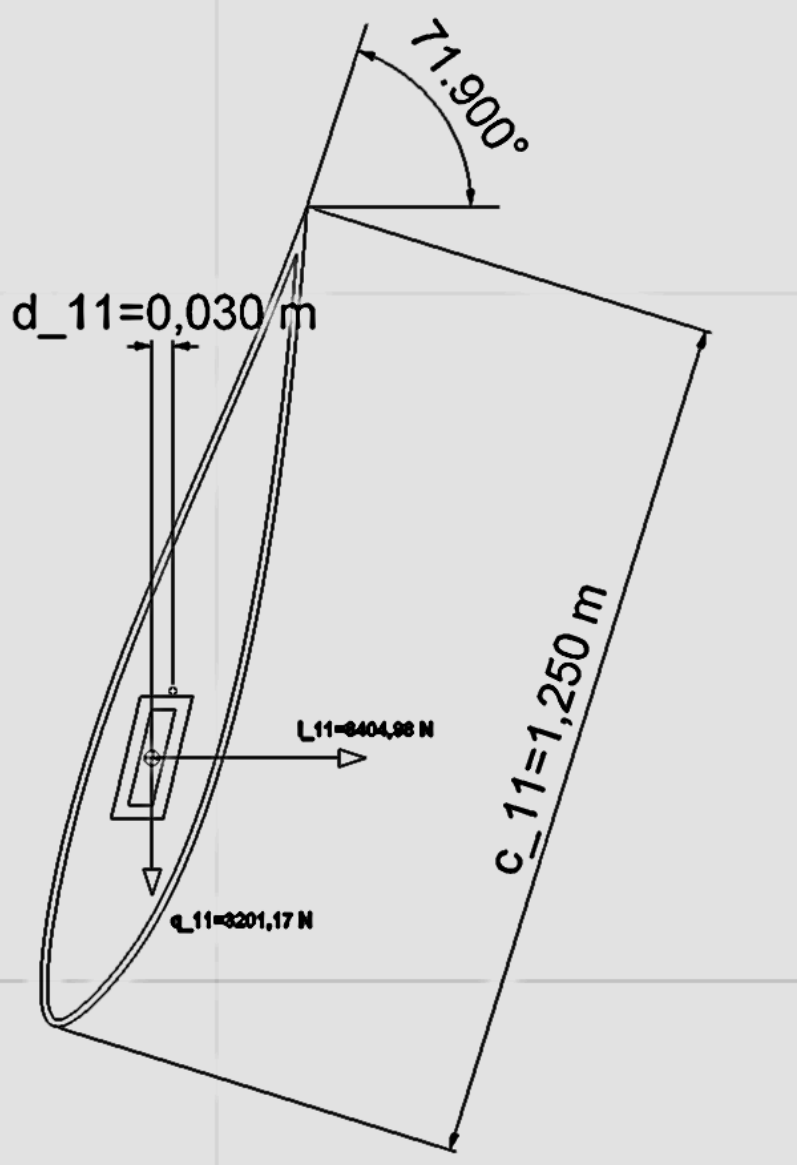

$$
\begin{gathered}
\frac{1}{2} \rho c_{m_{11}} c_{11}^{2} V_{11}^{2} \delta r_{11}=0,5 \cdot 1025,34 \cdot 0,0264 \cdot 1,250^{2} \cdot 7,466^{2} \cdot 0,45=530,46 \mathrm{Nm} \\
d_{11}\left(q_{11}+l_{11} \tan \beta_{11}\right)=0,030 \cdot(3201,17+8404,98 \cdot \tan 71,90)=867,487 \mathrm{Nm} \\
Q_{11}=530,46+867,487=1397,95 \mathrm{Nm}
\end{gathered}
$$


SECCIÓN No 12. $r_{12}=6,175 m ; \beta_{12}=72,90^{\circ} ; c_{12}=1,200 m ; N A C A 63-212$

\section{seccion 12}

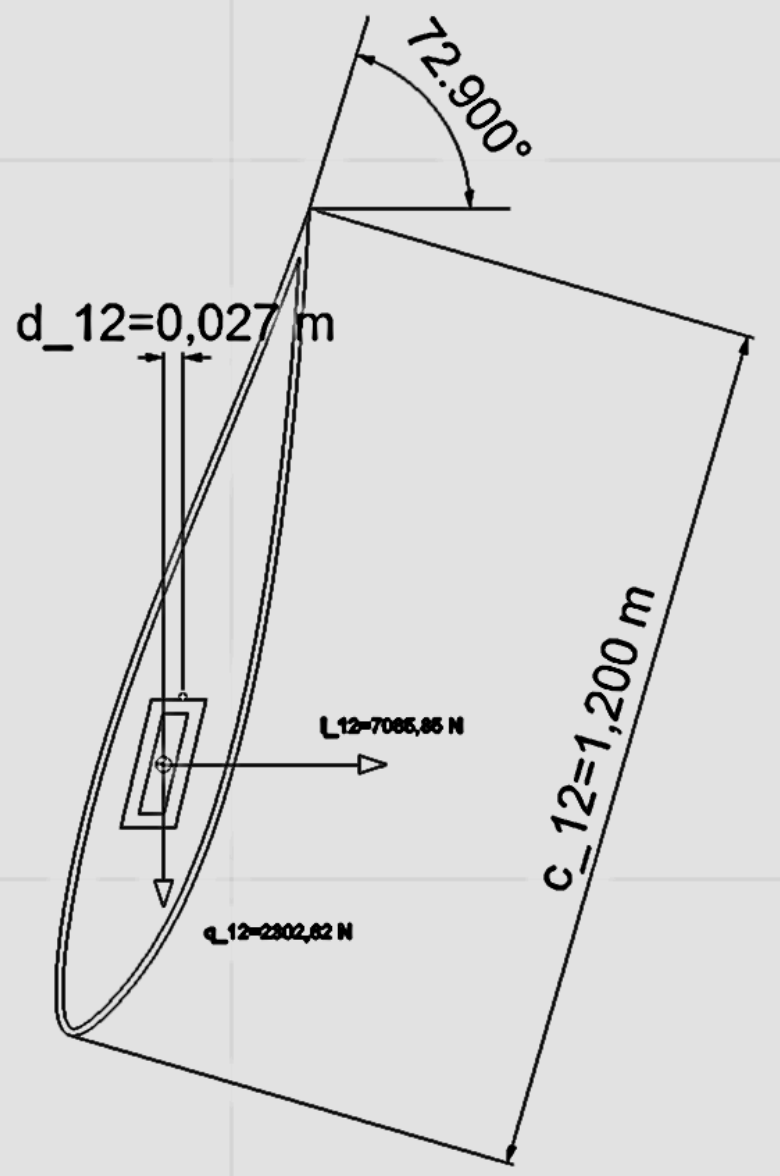

$$
\begin{gathered}
\frac{1}{2} \rho c_{m_{12}} c_{12}{ }^{2} V_{12}^{2} \delta r_{12}=0,5 \cdot 1025,34 \cdot 0,0317 \cdot 1,200^{2} \cdot 8,012^{2} \cdot 0,45=676,01 \mathrm{Nm} \\
d_{12}\left(q_{12}+l_{12} \tan \beta_{12}\right)=0,027 \cdot(2302,62+7085,85 \cdot \tan 72,90)=684,06 \mathrm{Nm} \\
Q_{12}=676,01+684,06=1360,07 \mathrm{Nm}
\end{gathered}
$$


SECCIÓN No 13. $r_{13}=6,625 m ; \beta_{13}=73,90^{\circ} ; c_{13}=1,150 m ; N A C A 63-212$

\section{seccion 13}

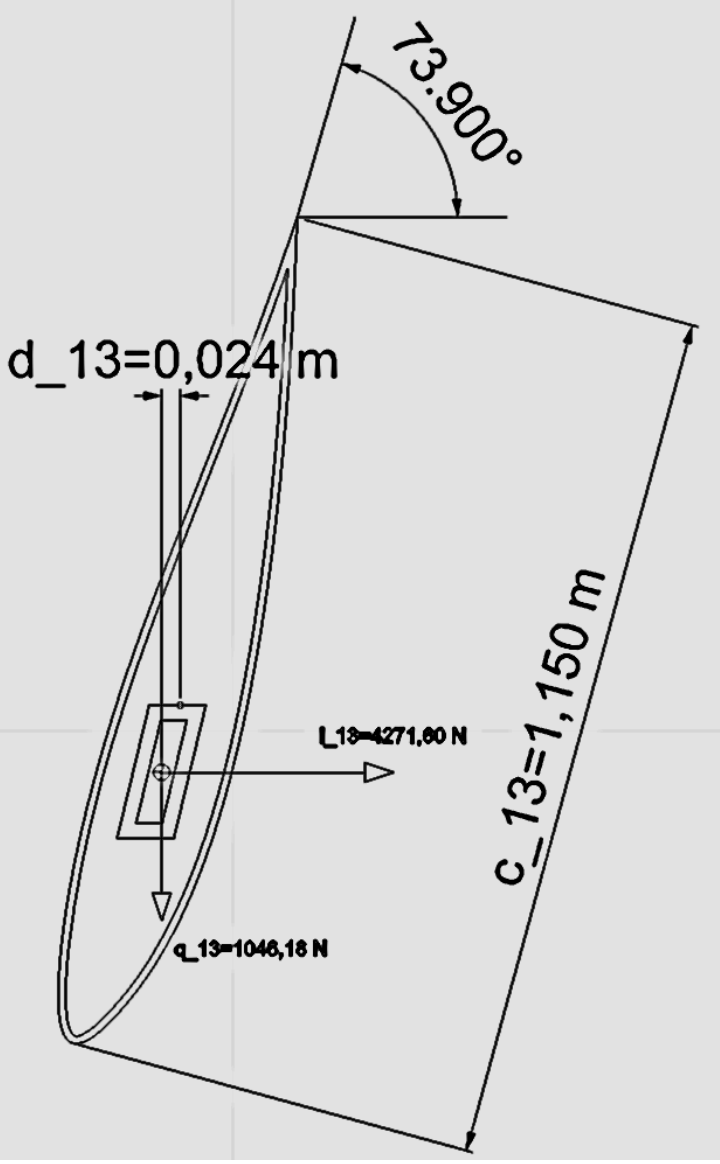

$$
\begin{gathered}
\frac{1}{2} \rho c_{m_{13}} c_{13}^{2} V_{13}^{2} \delta r_{13}=0,5 \cdot 1025,34 \cdot 0,0369 \cdot 1,150^{2} \cdot 8,561^{2} \cdot 0,45=825,13 \mathrm{Nm} \\
d_{13}\left(q_{13}+l_{13} \tan \beta_{13}\right)=0,024 \cdot(1046,18+4271,60 \cdot \tan 73,90)=380,29 \mathrm{Nm} \\
Q_{13}=825,13+380,29=1205,42 \mathrm{Nm}
\end{gathered}
$$


SECCIÓN No 14. $r_{14}=7,075 m ; \beta_{14}=74,90^{\circ} ; c_{14}=1,100 m ; N A C A 63-212$ seccion 94

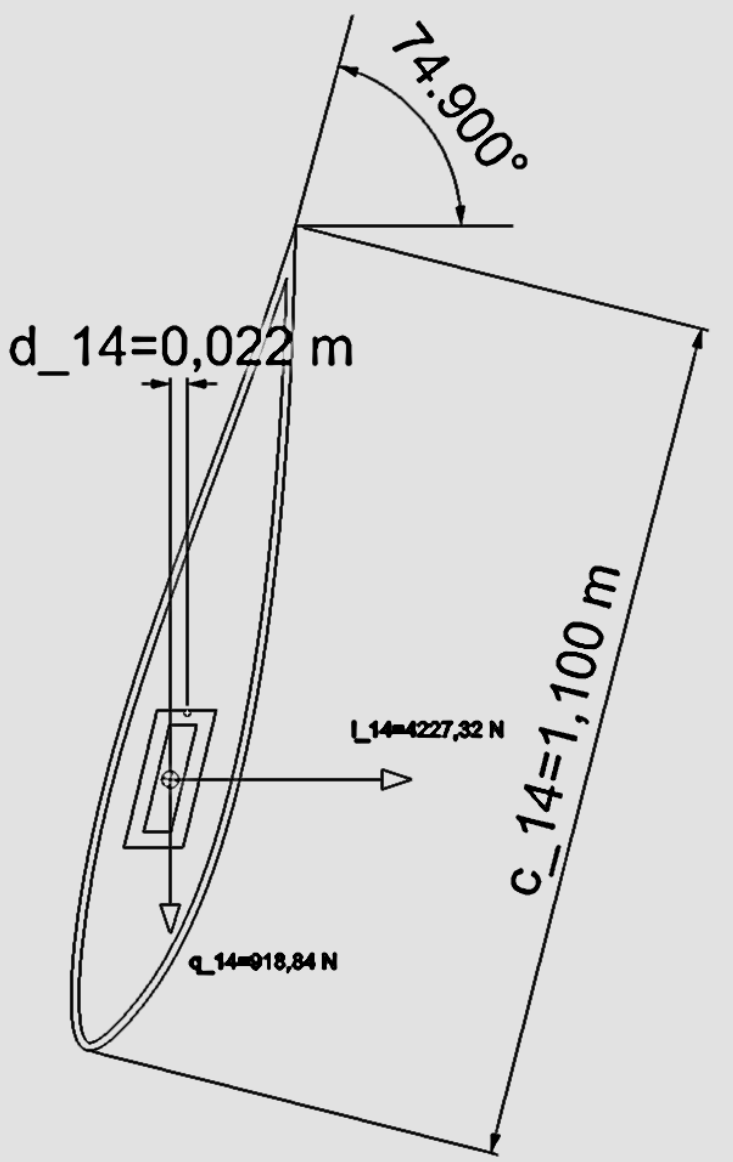

$$
\begin{gathered}
\frac{1}{2} \rho c_{m_{14}} c_{14}{ }^{2} V_{14}^{2} \delta r_{14}=0,5 \cdot 1025,34 \cdot 0,0374 \cdot 1,100^{2} \cdot 9,111^{2} \cdot 0,45=866,64 \mathrm{Nm} \\
d_{14}\left(q_{14}+l_{14} \tan \beta_{14}\right)=0,022 \cdot(918,84+4227,32 \cdot \tan 74,90)=364,89 \mathrm{Nm} \\
Q_{14}=866,64+364,89=1231,53 \mathrm{Nm}
\end{gathered}
$$


SECCIÓN No 15. $r_{15}=7,525 m ; \beta_{15}=75,90^{\circ} ; c_{15}=1,050 m ; N A C A 63-212$
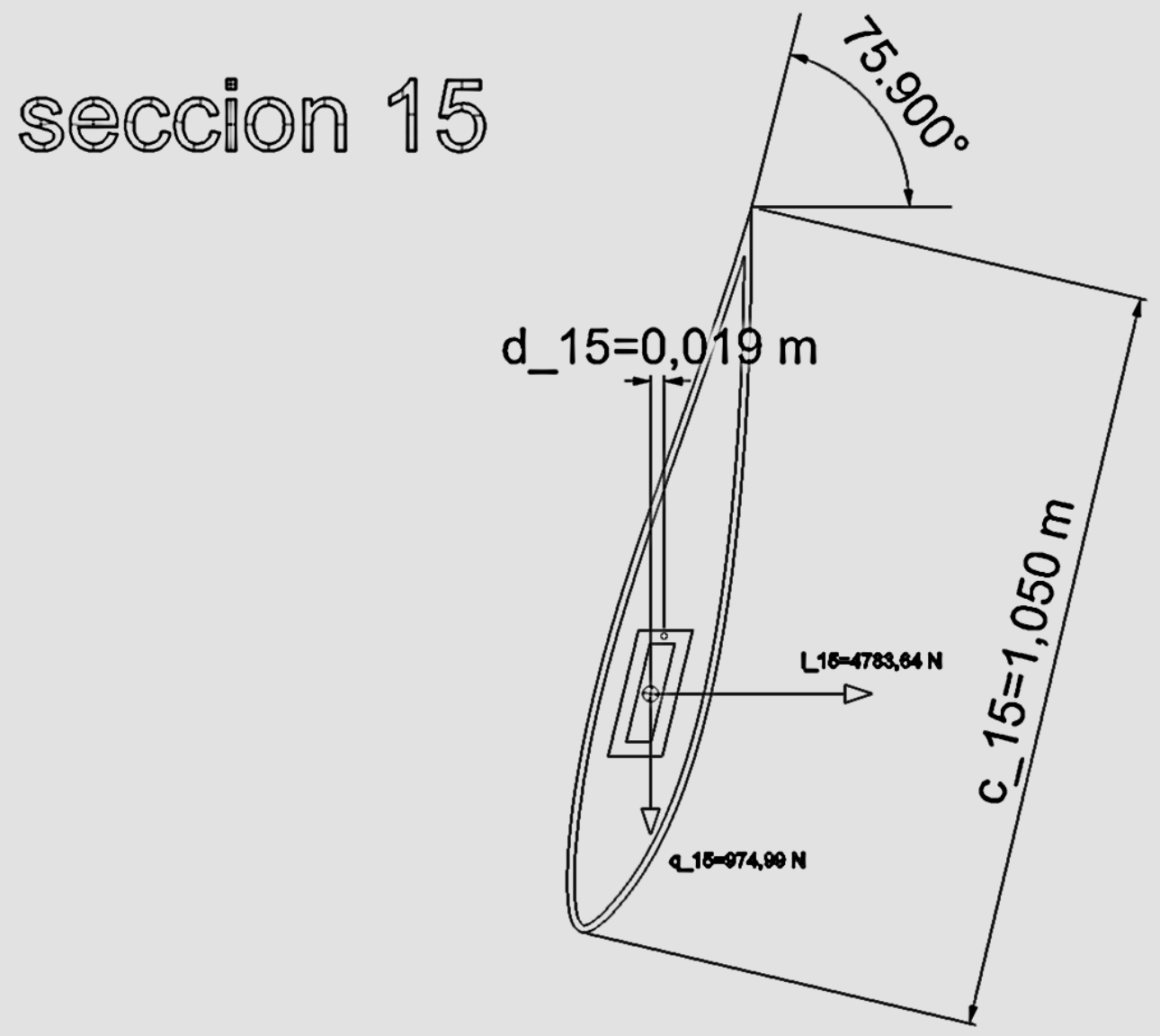

$$
\begin{gathered}
\frac{1}{2} \rho c_{m_{15}} c_{15}{ }^{2} V_{15}^{2} \delta r_{15}=0,5 \cdot 1025,34 \cdot 0,0371 \cdot 1,050^{2} \cdot 9,664^{2} \cdot 0,45=881,29 \mathrm{Nm} \\
d_{15}\left(q_{15}+l_{15} \tan \beta_{15}\right)=0,019 \cdot(974,99+4783,64 \cdot \tan 75,90)=380,37 \mathrm{Nm} \\
Q_{15}=881,29+380,37=1261,66 \mathrm{Nm}
\end{gathered}
$$


SECCIÓN No 16. $r_{16}=7,975 m ; \beta_{16}=76,40^{\circ} ; c_{16}=0,975 m ; N A C A 63-212$

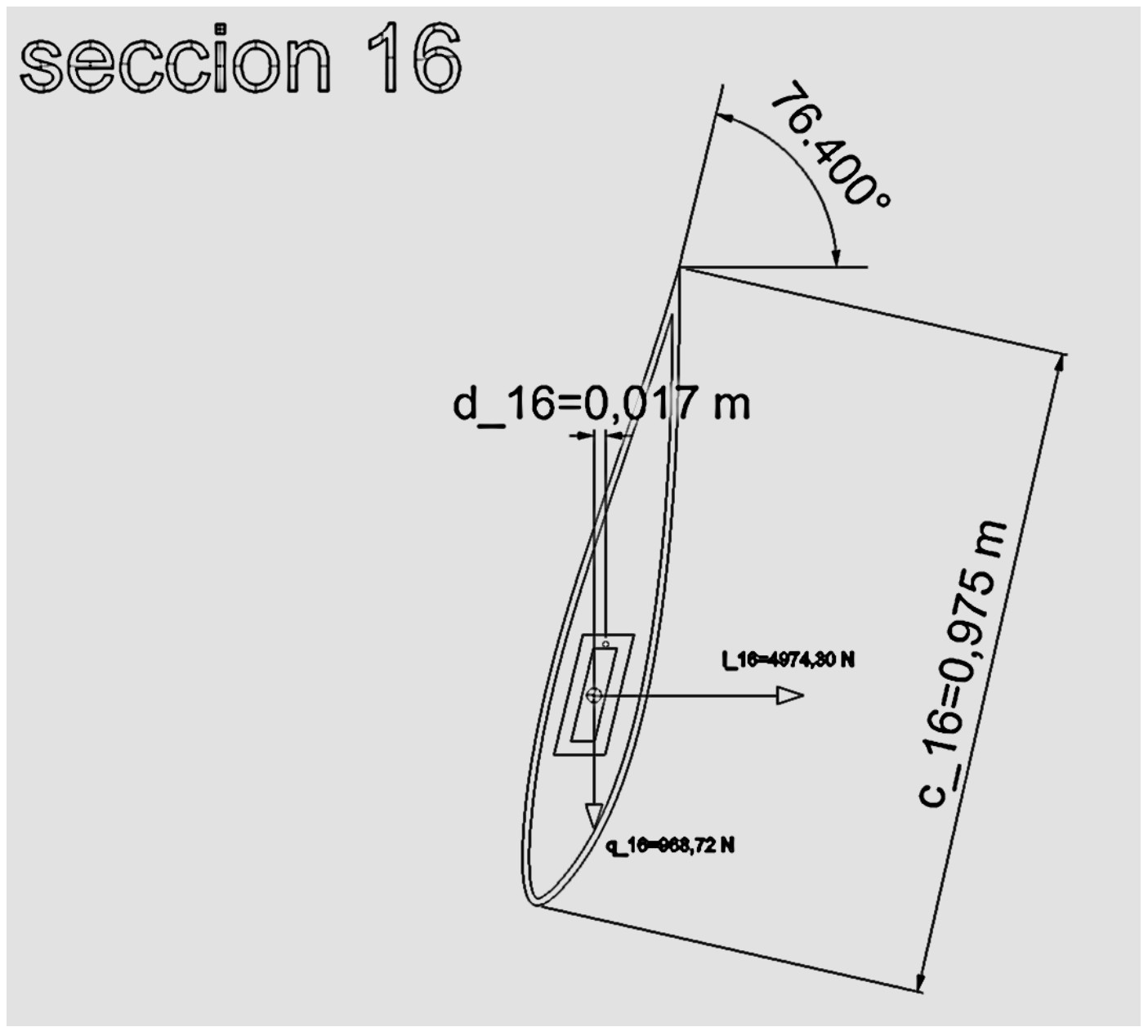

$$
\begin{gathered}
\frac{1}{2} \rho c_{m_{16}} c_{16}{ }^{2} V_{16}^{2} \delta r_{16}=0,5 \cdot 1025,34 \cdot 0,0371 \cdot 0,975^{2} \cdot 10,217^{2} \cdot 0,45=849,33 \mathrm{Nm} \\
d_{16}\left(q_{16}+l_{16} \tan \beta_{16}\right)=0,017 \cdot(968,72+4974,30 \cdot \tan 76,40)=366,01 \mathrm{Nm} \\
Q_{16}=849,33+366,01=1215,34 \mathrm{Nm}
\end{gathered}
$$


SECCIÓN No 17. $r_{17}=8,425 m ; \beta_{17}=76,90^{\circ} ; c_{17}=0,900 m ; N A C A 63-212$

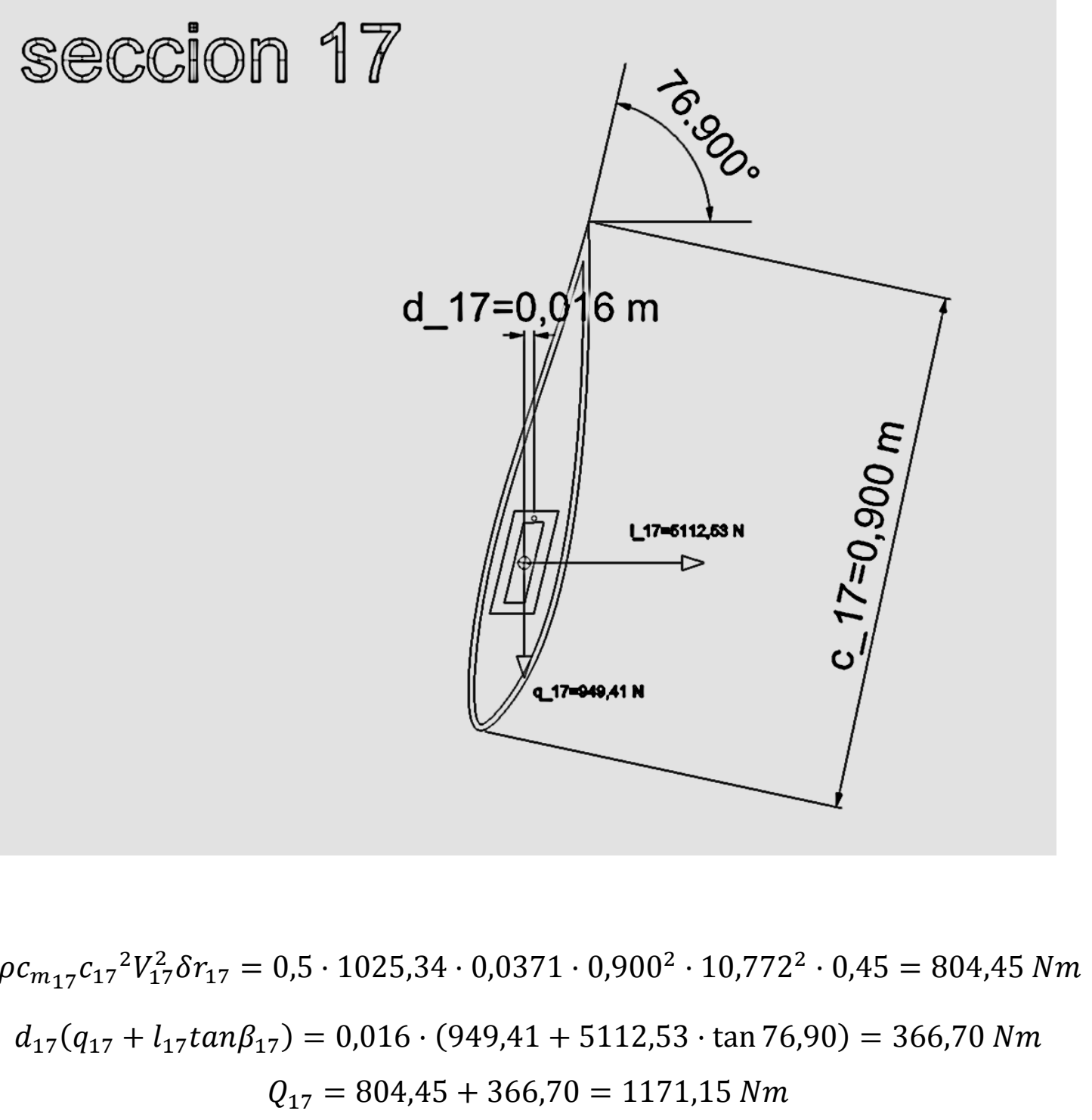


SECCIÓN No 18. $r_{18}=8,875 m ; \beta_{18}=76,90^{\circ} ; c_{18}=0,800 m ; N A C A 63-212$ seccion 18

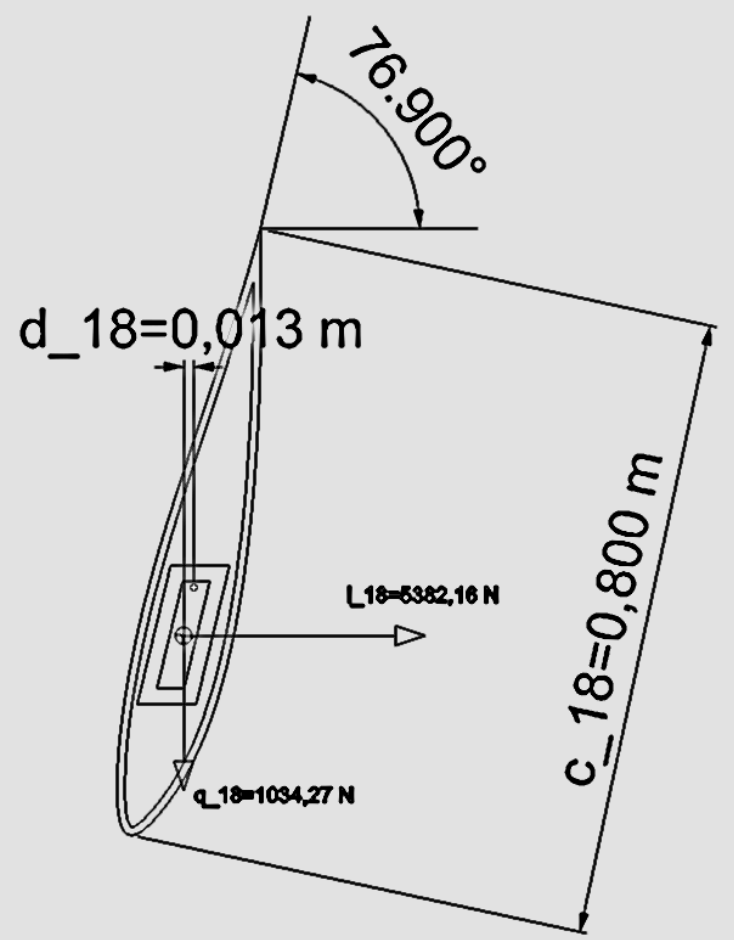

$$
\begin{gathered}
\frac{1}{2} \rho c_{m_{18}} c_{18}{ }^{2} V_{18}^{2} \delta r_{18}=0,5 \cdot 1025,34 \cdot 0,0369 \cdot 0,800^{2} \cdot 11,328^{2} \cdot 0,45=699,13 \mathrm{Nm} \\
d_{18}\left(q_{18}+l_{18} \tan \beta_{18}\right)=0,013 \cdot(1034,27+5382,16 \cdot \tan 76,90)=314,12 \mathrm{Nm} \\
Q_{18}=699,13+314,12=1013,25 \mathrm{Nm}
\end{gathered}
$$


SECCIÓN No 19. $r_{19}=9,325 m ; \beta_{19}=76,90^{\circ} ; c_{19}=0,700 m ; N A C A 63-212$

\section{Seccion 79}

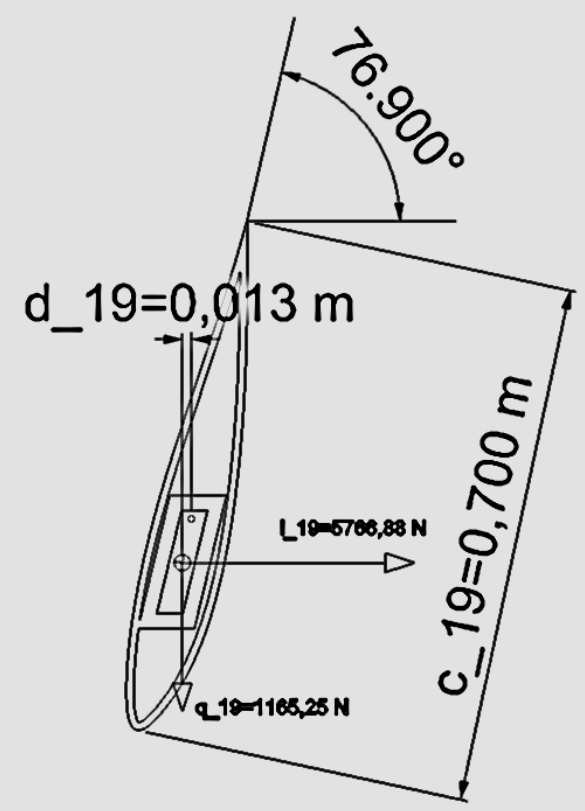

$$
\begin{gathered}
\frac{1}{2} \rho c_{m_{19}} c_{19}{ }^{2} V_{19}^{2} \delta r_{19}=0,5 \cdot 1025,34 \cdot 0,0364 \cdot 0,700^{2} \cdot 11,885^{2} \cdot 0,45=581,23 \mathrm{Nm} \\
d_{19}\left(q_{19}+l_{19} \tan \beta_{19}\right)=0,013 \cdot(1165,25+5766,88 \cdot \tan 76,90)=337,31 \mathrm{Nm} \\
Q_{19}=581,23+337,31=918,54 \mathrm{Nm}
\end{gathered}
$$


SECCIÓN No 20. $r_{20}=9,775 m ; \beta_{20}=76,90^{\circ} ; c_{20}=0,550 m ; N A C A 63-212$

\section{seccion 20}

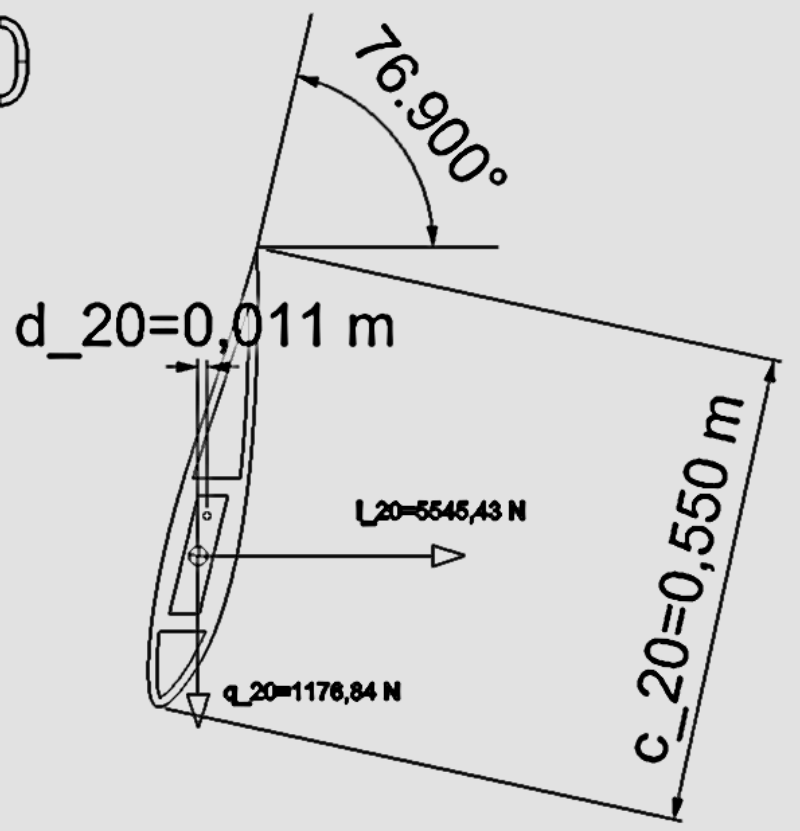

$\frac{1}{2} \rho c_{m_{20}} c_{20}{ }^{2} V_{20}^{2} \delta r_{20}=0,5 \cdot 1025,34 \cdot 0,0356 \cdot 0,550^{2} \cdot 12,443^{2} \cdot 0,45=384,66 \mathrm{Nm}$

$$
\begin{gathered}
d_{20}\left(q_{20}+l_{20} \tan \beta_{20}\right)=0,011 \cdot(1176,84+5545,43 \cdot \tan 76,90)=275,08 \mathrm{Nm} \\
Q_{20}=384,66+275,08=659,74 \mathrm{Nm}
\end{gathered}
$$

